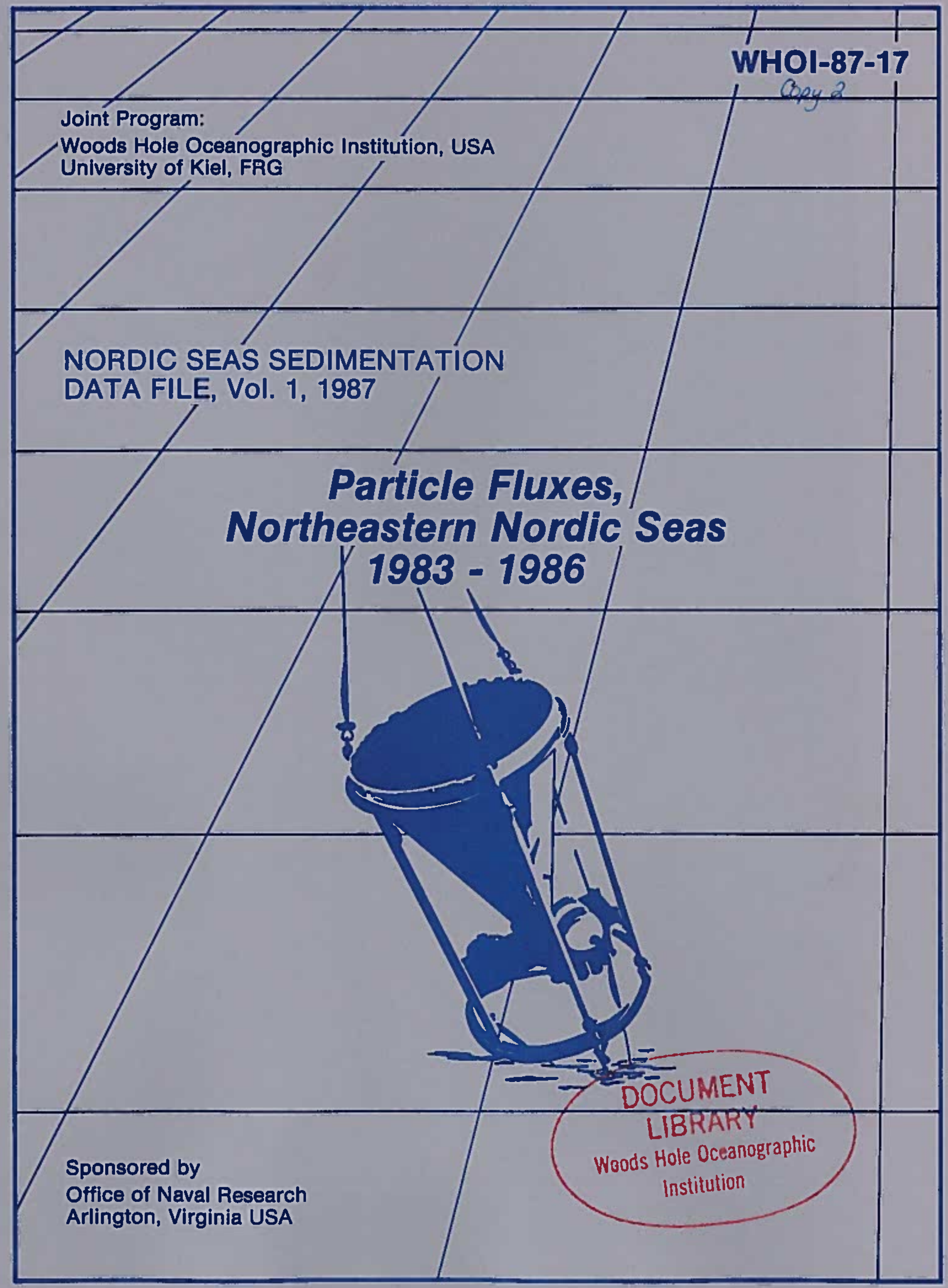


WHOI $-87-17$

Particle Fluxes, North-Eastern Nordic Seas: 1983-1986

(Nordic Seas Sedimentation Data File, Vo1. 1)

by

Susumu Honjo, Steven J. Manganini, Amy Karowe, Bonnie L. Woodward

Woods Hole Oceanographic Institution

Woods Hole, Massachusetts 02543

Apri1, 1987

Technical Report

Funding was provided by the Office of Naval Research under Grant Number N00014--85-C-0001.

Reproduction in whole or in part is permitted for any purpose of The United States Government. This report should be cited as:

Woods Hole Oceanog. Inst. Tech. Rept. WHOI-87-17.

Approved for publication; distribution unlimited

Approved for Distribution:

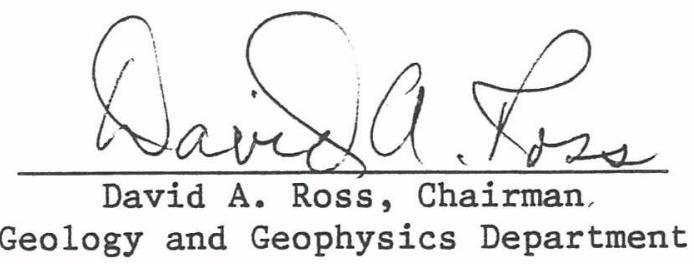




\title{
Joint Program:
}

\author{
Woods Hole Oceanographic Institution \\ and \\ University of Kiel, University of Bremen, FRG
}

NORDIC SEAS SEDIMENTATION

DATA FILE, Vo1. 1

PARTICLE FLUXES,

NORTH-EASTERN NORDIC SEAS:

$1983-1986$

Sponsored by Office of Naval Research Arlington, Virginia USA 
Table of Contents

Page

Abstract . . . . . . . . . . . . . . . . . 1

Introduction ....................... 1

Field Program ..................... 3

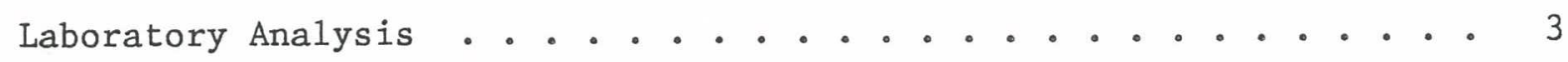

Results ....................... 4

Acknowledgments . . . . . . . . . . . . . . . 5

References ...................... 6

Table 1. Sediment trap moorings, 1983-1987 . . . . . . . . . 7

Table 2. Average mass fluxes in the northern Nordic Seas, 1983-1986 . 10

Table 3. Comparison of mass fluxes between stations in the northern

Nordic Seas, 1983-1986 . . . . . . . . . . 11

Figure 1. Location of map of sediment trap moorings . . . . . . . 12

Figure 2. PARFLUX Mark 5 and 6 sediment traps ........... 13

Figure 3. Analytical procedure . . . . . . . . . . . . . 14

Flux Data Files:

Norwegian-Atlantic Current Area . . . . . . . . . . . 15

LB-1 East Lofoten Basin . . . . . . . . . . 16

BI-1 Bear Island-West of Starfjord ............ 26

NA-1 Aegir Ridge ................. 36

NB-1 East of Jan Mayen .............. 46

East Greenland/Fram Strait Area . . . . . . . . . . . 56

FS-1 Central Fram Strait . . . . . . . . . . 57

GB-2.2K Greenland Basin, 1,966 m .............. 67

GB-2.3K Greenland Basin, $2,817 \mathrm{~m}$. ............. 75 


\title{
Nordic Seas Sedimentation Data File, Volume 1 \\ Particle Fluxes, North-Eastern Nordic Seas: 1983 - 1986
}

\author{
by \\ Susumu Honjo, Steven J. Manganini, Amy Karowe, Bonnie L. Woodward \\ Woods Hole Oceanographic Institution \\ Woods Hole, Massachusetts 02543
}

Apri1, 1987

\section{Abstract}

Seventy-nine particle flux samples were collected from 1983 to 1986 using 7 automated time-series sediment traps at 6 stations distributed in the northern and eastern portion of the Nordic Seas as part of a German/U.S. joint program on arctic sedimentation studies. Each sample represents either one month or two weeks of sedimentation at approximately $400 \mathrm{~m}$ above the sea floor. In this data file the results of laboratory analysis conducted at the Woods Hole Oceanographic Institution, U.S.A. of the main sedimentological criteria: total mass, carbonate, opal, combustible, organic carbon, nitrogen, and lithogenic mass are presented in both tabular and histogram form. Results from the southern and western portion of the Nordic Seas will be published as they become available.

\section{Introduction}

Supported by the United States Office of Naval Research, the Woods Hole Oceanographic Institution (WHOI), with the cooperation of the University of Kiel and the University of Bremen, Federal Repub1ic of Germany, has conducted a basin-wide sedimentological research program in the Nordic Sea since the summer of 1983. One of the major field experiments was deployment of 16 sets of sediment trap-current meter moorings for a period of about one year each throughout the basin. During the first half of the program we deployed 6 year-round moorings between August 1983 and August 1986 in the Fram Strait and Norwegian Basin. Details of mooring positions, depths, duration of deployment are summarized in Table 1. During the second part of the program, sediment trap mooring deployments and laboratory analyses of incoming samples will continue around Iceland, coastal Greenland and selected stations in cooperation with the Marine Research Institute, Reykjavik. The 
University of Hamburg maintains 3 sediment trap mooring stations in the southern North Sea and we cooperate with their program on some of the laboratory analyses (Fig. 1).

The Nordic Sea is a basin, approximately 2.5 million square kilometers, defined by the east coast of Greenland to the west, Iceland to the south, the Norwegian coast to the east, and Spitsbergen to the north. It connects to the Arctic Ocean via the Fram Strait and to the North Atlantic via the Faeroe and Denmark straits (Hurdle, 1986). In short, the Nordic Sea is the bridge between the Arctic Ocean and the North Atlantic 0cean, and therefore is of global significance in regard to the Atlantic environment.

Most of the Nordic Sea lies north of the Arctic Circle. The net solar energy input is strongly limited in this basin due to low angle insolation during the summer and day-1ong darkness in the winter. Three longitudinal zones of ocean characteristics can be distinguished in this basin: 1) a zone along the east coast of Greenland which is covered by southerly flowing ice packs and floes in the East Greenland Current combined with fast-ice conditions on the immediate coast (Vinje, 1977). The surface temperature in this zone is $0^{\circ} \mathrm{C}$ throughout the year; 2) a zone on the east side of the basin where the warm, saline northward-flowing Norwegian-Atlantic Current prevails (Gathman, 1986); and 3 ) a zone in the central gyre which is often associated with mixed ice conditions where the other two zones meet in the middle of the basin (Wadhams, 1986; Swift, 1986). This unique arrangement of currents form several ocean fronts (Johannessen, 1986) and strong contrasts of oceanic conditions are seen within this relatively small basin. For example, the summer surface temperature difference between the east and west side of the basin along the 70th latitude (off Tromso, Norway to Scoresby Sound, Greenland, which are only about $1,000 \mathrm{~km}$ apart) is as great as $10^{\circ} \mathrm{C}$ in some years (Detrich, 1969). Thus the Nordic Sea embodies highly diversified specific environments within the basin boundary.

Very little is known about particle sedimentation and recycling schemes in the North Sea environment. Ocean particles in the Nordic Basin also involve specific origins, flux and processes which reflect varied oceanic characteristics. Questions include: how much of the particulate carbon and other biogenic particles settle down to the sea floor and how do they compare with surface production which is produced under severely limiting Arctic conditions? What is the sedimentary mechanism of lithogenic particles in the Arctic open ocean environment? How are these sedimentary particle processes related to ice coverage and mixed ice zone conditions? This research aims to answer these questions and, optimally, to draw a realistic model of particle flux and sedimentation in relation to other critical high latitude ocean environmental factors. 
Field Program

Experimental logistics in the Nordic Sea are generally very difficult compared to lower latitude oceanographic endeavors; winter storms and ice coverage hinder deployment and recovery of large bottom tethered mooring arrays. Because of strong seasonality, flux measurements in high latitudes must cover at least a one-year cycle of seasons. We have used automated time-series sediment traps left unattended for about one year. A sediment trap used in this environment requires a large opening in order to collect enough volume of sample during the winter months when the flux is estimated to be extremely sma11. We used a PARFLUX Mark 5 and Mark 6 whose apertures are 1.2 and $0.5 \mathrm{~m}^{2}$ with 12 and 13 sampling increments, respectively (Honjo and Doherty, 1987, in press) (Table 1, Fig. 2). The sediment traps were deployed at approximately $400 \mathrm{~m}$ above the sea floor at most mooring sites. The exception was a mooring with two sediment traps deployed along a taut line which was set in the Greenland Basin. One to three current meters were deployed with each sediment trap mooring. A transmissometer was deployed with two Fram Strait moorings for one year, 1984-1985. The results from the current meter and transmissometer experiments will be published elsewhere. The deployment/recovery procedure for sediment trap mooring arrays was described in a separate paper (Honjo and Doherty, 1987, in press) We used sodium azide as a preservative (Honjo, 1980).

Laboratory Analysis

Recovered samples were refrigerated throughout the transportation and storage period. Each sample was equally shared with Dr. Gerold Wefer's laboratory (University of Bremen). Our responsibility at WHOI was to clarify the nature of the sediment trap collected samples with regard to basic sedimentological criteria. Dr. Wefer's group is investigating stable isotopes in planktonic foraminiferal tests and some biocoenosis composition in the samples.

Upon arrival at WHOI, each sample was sieved through a $1 \mathrm{~mm}$ Nylon mesh. This was necessary to maintain precise sample splitting.

Particles smaller than $1 \mathrm{~mm}$ were further split into smaller aliquots by a precision wet sample splitter (Honjo, 1980). The split aliquots were further sieved through a 62 micron mesh for the LB-1, FS-1, and BI-1 samples in order to separate foraminiferal tests and radiolarian shells in this size category more efficiently. We analyzed individually samples in each size category for the following criteria. All results were normalized to flux values in $\mathrm{mg} \mathrm{m}^{2}$ day (Honjo, 1980).

Tota1 mass

Carbonate mass

Combustible mass 
Noncombustible mass

Opa1 mass

Lithogenic mass

Organic carbon, nitrogen, and hydrogen mass

A detailed description of the analytical methods applied to this research will be published elsewhere. In summary as illustrated in Fig. 3 , the total mass flux was obtained as the average of dry mass weight of the three 16th aliquots. The carbonate content was obtained from the dry weight difference before and after decalcification by $1 \mathrm{~N}$ acetic acid at room temperature. A decalcified aliquot was combusted for 3 hours at $500^{\circ} \mathrm{C}$ to obtain the mass of combustible organic matter as the difference between a decalcified sample and ash weight. Biogenic silica, or opal, content was analyzed by the sodium carbonate leaching method modified from Eggiman et al., 1980, on decalcified aliquots. Lithogenic particle flux, mostly clay and fine rock-forming detritus, was gained by subtracting the opal flux from the noncombustible flux. Organic carbon, nitrogen, and hydrogen content were analyzed using a Perkin-Elmer Elemental Analyzer, type 240C. We used at least $100 \mathrm{mg}$ of decalcified samples (Fig. 3).

Total flux, therefore, is equal to the sum of carbonate, noncombustible, and combustible fluxes. The sum of biogenic opal and 1ithogenic fluxes should be the noncombustible flux. Insignificant discrepancies appear in some total flux values in this data file due to the rounding out processes during calculation. We regard the combustible portion of the flux as organic matter flux (Honjo, 1980). Combustible flux consists of organic carbon, nitrogen, and hydrogen balanced with oxygen and other unidentifiable ignition loss. The amount of organic nitrogen in the GB-1 sample was too small to analyze within our level of confidence. The opal content in the GB-2, 1966 trap sample was also too small to analyze with the leaching method at the time but we are making an effort to bring up significant numbers.

The phosphorus flux from this area will be published in a separate file. The results of analysis of 15 trace elements from all time-series sediment trap samples treated in the present data file (total of 1,185 analyses) will be published in a separate volume.

\section{Results}

The purpose of this data file is to publish a summary of available data on the flux in the north-eastern Nordic Sea for public use. Scientific interpretations and models will not be included in this publication. 
The annual averages in two major areas, Norwegian-Atlantic current area and the East Greenland current area (sea ice prevailed) based upon fluxes from 6 stations presented in this report, is given in Table 2. The annual fluxes of sedimentary components from 6 stations are tabulated in Table 3 for comparison. At the beginning of each data file for individual stations are given the sample identification numbers, opening and closing dates, length of collection period and mid-point date during which the samples were collected. On subsequent pages are given the percentages of total flux of three size categories: particles which passed through 62 micrometer mesh $(<63 \mu \mathrm{m})$, particles retained in a 1 $\mathrm{mm}$ mesh (>1 $1 \mathrm{~mm})$, and particles in between $(63 \mu \mathrm{m}-1 \mathrm{~mm})$. In the rightmost column of the table, the total flux of size categories combined is given. The columns of each histogram are labeled according to mid-point day of the sampling period. The six flux categories listed in the previous section are included in each data set.

\section{Acknowledgments}

Without the encouragement and support of Dr. G. Leonard Johnson, office of Naval Research, this first entire ocean basin sedimentation study applying the flux concept would never have been started. We sincerely thank him for his insight and strong commitment to excellent science.

The Nordic Sea is one of the most difficult oceans with regard to experimental logistics. We have received a large amount of good will support from international colleagues; a large part of our success is due to them and even the unusually long acknowledgment in this paper may cover only a portion. In particular, the Alfred Wegener Institution of Polar and Marine Research, Bremerhaven provided us with vital shiptime on board R/V Polarstern for this experiment. Dr. Jörn Thiede, Chief Scientist of the 1984 and 1985 legs, took every possible opportunity to help us with his professional competence and personal care during this experiment. We also thank the $R / V$ Meteor (old) and the Deutche Hydrographische Institute, Hamburg, which supported us in a difficult mission to recover a malfunctioned mooring system and to deploy a large array in the Greenland Sea during the summer of 1985. We also thank the $\mathrm{R} / \mathrm{V}$ Meteor (new) and $\mathrm{R} / \mathrm{V}$ Valdivia, University of Hamburg, for their high quality support of the mooring experiments in 1986.

The Nordic Sea program has been carried out under the mutual cooperation among the University of Bremen, University of Kiel and Woods Hole Oceanographic Institution. Dr. Gerold Wefer, our partner, has provided many useful suggestions in research and has been very helpful in providing vital logistic support. We own him our sincere gratitude. We thank for their dedication and imagination: Dr. Vernon L. Asper, 
University of Southern Mississippi, and Dorinda Ostermann, WHOI, who made it possible to deploy and recover the first 4 mooring arrays in the northern Nordic Sea in 1983 and 1984; Peter Clay and Thomas Crook who provided vital assistance in recovering a stranded GB-1 mooring in the summer of 1985; Emily Evans who took care of communication traffic and data editing during this program.

\section{References}

Detrich, G., 1969. Atlas of the Hydrography of the Northern North Atlantic, International Council for the Exploration of the Sea. Copenhagen.

Eggimann, D.W., Manheim, F.T. and Betzer, P.R., 1980. Dissolution and Analysis of Amorphous Silica in Marine Sediments. Journal of Sedimentary Petrology, 50(1): 215-225.

Gathman, S.G., 1986. Climatology. In: The Nordic Seas (Hurdle, B.G., ed.), 1-18, Springer-Verlag, New York, 777 pp.

Hurdle, B.G. (ed.), 1986. The Nordic Seas. Springer-Verlag, New York, 777 pp.

Honjo, S., 1980. Material Fluxes and Modes of Sedimentation in the Mesopelagic and Bathypelagic zones. Journal of Marine Research, 38: 53-97.

Honjo, S. and Doherty, K.W., 1987 (in press). Large Aperture Time-Series Oceanic Sediment Traps; Design Objectives, Construction and Applications. Deep-Sea Research.

Johannessen, 0., 1986. Brief overview of Physical Oceanography. In: The Nordic Seas (Hurdle, B.G., ed.), 103-127, Springer-Verlag, New York, $777 \mathrm{pp}$.

Swift, J.H., 1986. The Arctic Waters. In: The Nordic Seas (Hurdle, B.G., ed.), 129-153, Springer-Verlag, New York, 777 pp.

Vinje, T.E., 1977. Sea Ice Conditions in the European Sector of the Marginal Sea of the Arctic, 1966-75. Norsk Polarinstitutt Arbok, 1975: 163-174.

Wadhams, P., 1986. The Ice Cover. In: The Nordic Seas (Hurdle, B.G., ed.), 21-84, Springer-Verlag, New York, 777 pp. 


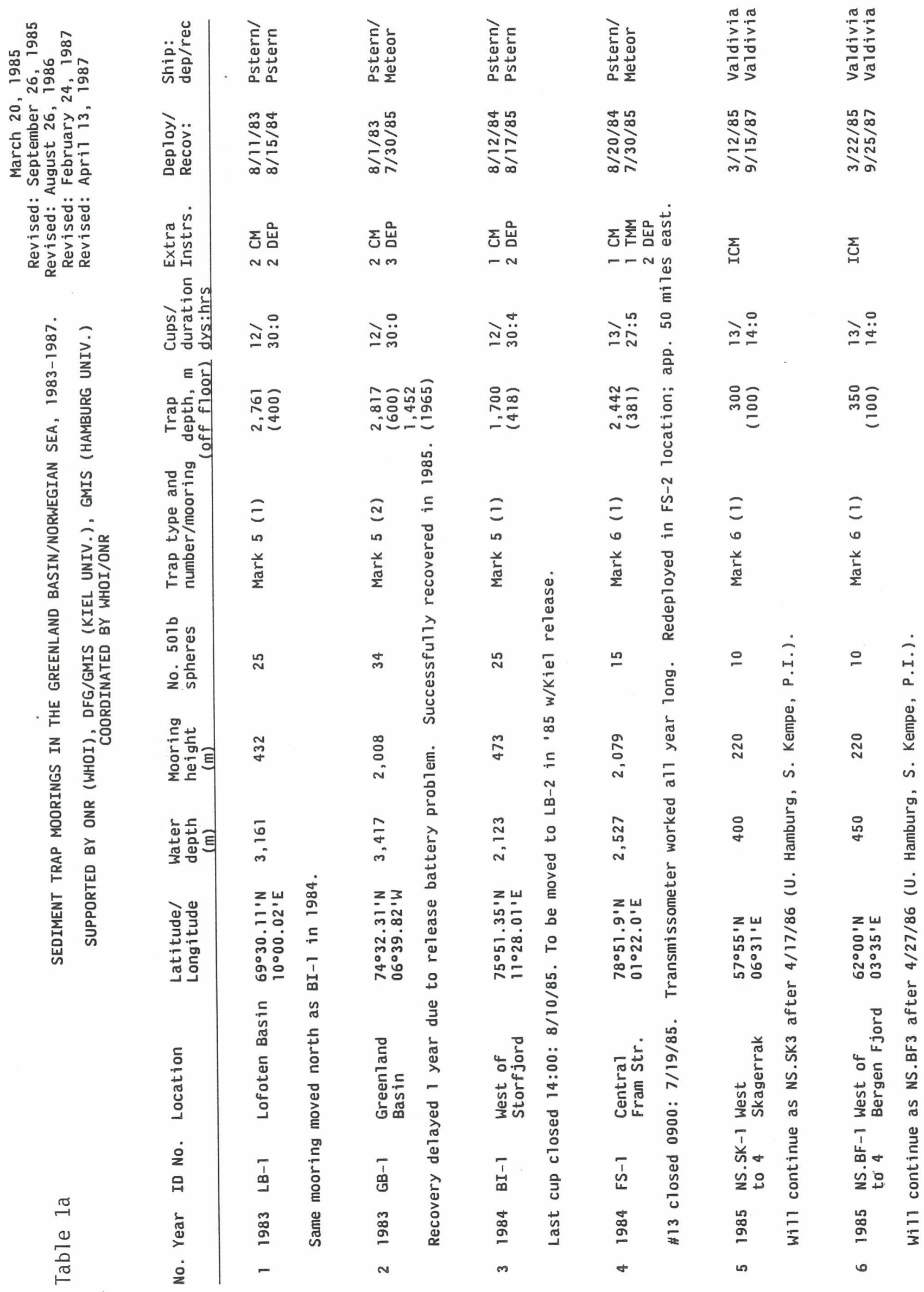




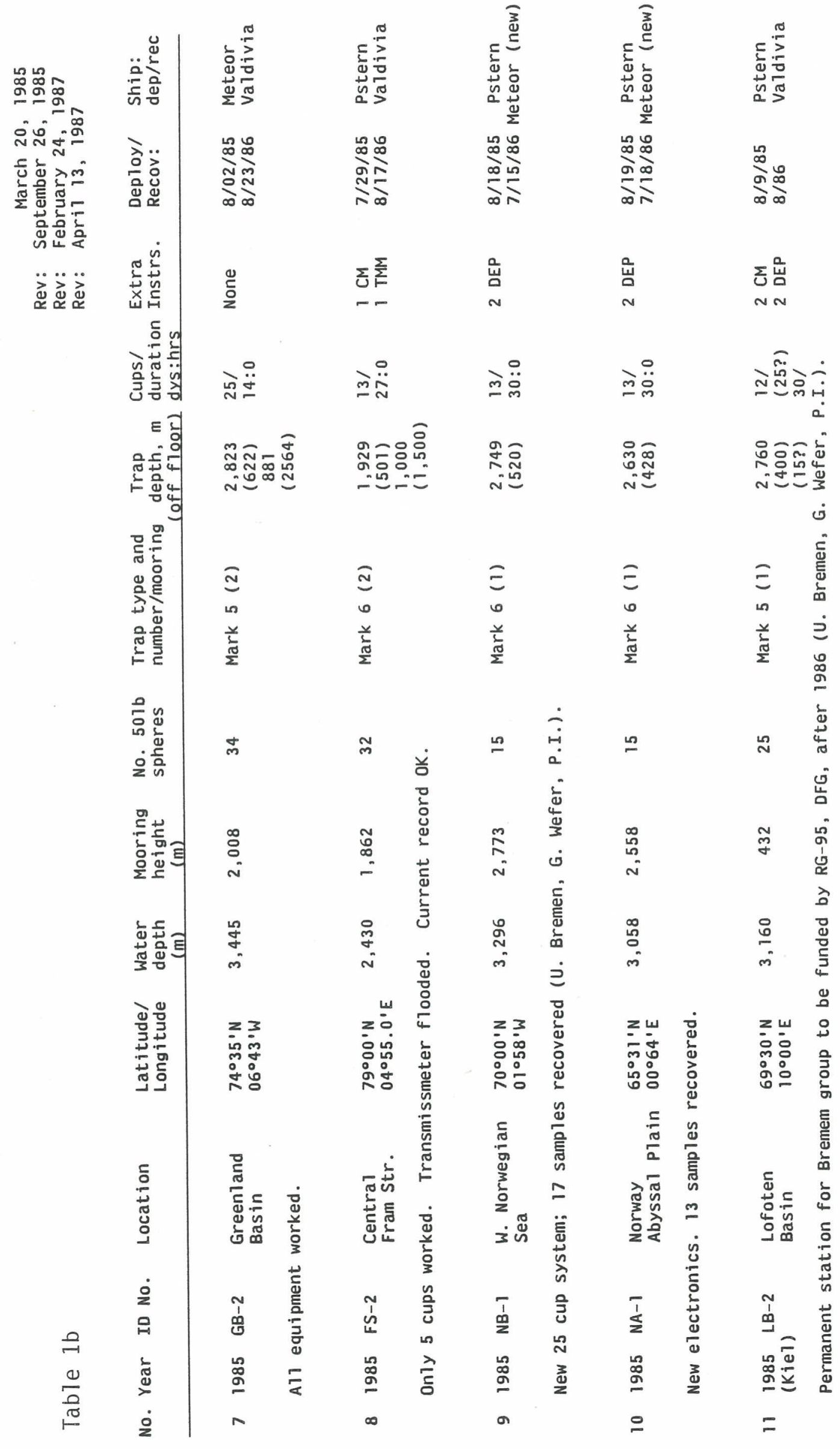




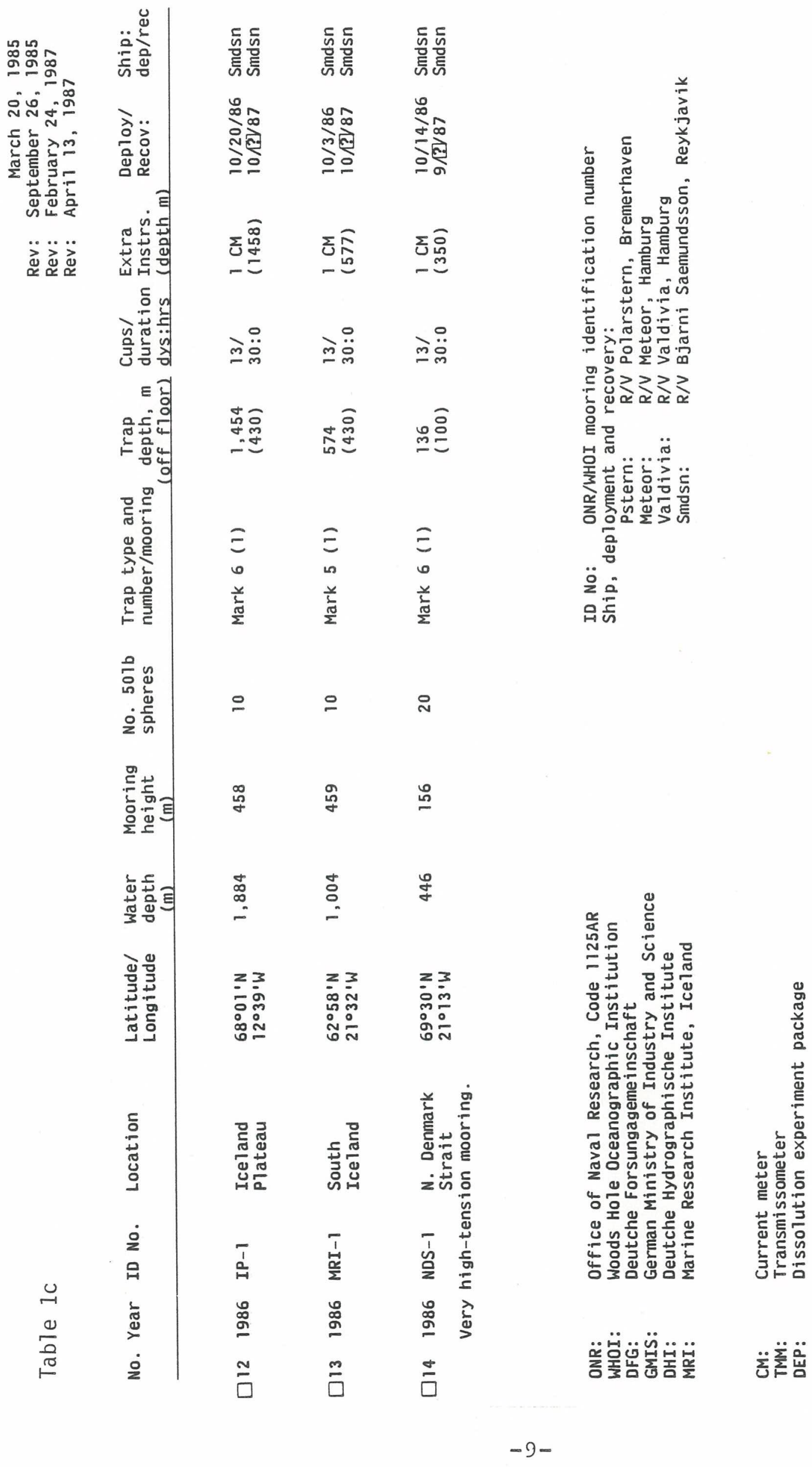


Table 2. Average Mass Fluxes; Northern Nordic Seas, 1983-1986. Average Fluxes and (standard deviation) $\mathrm{mg} \mathrm{m}^{-2} \mathrm{day}^{-1}$.

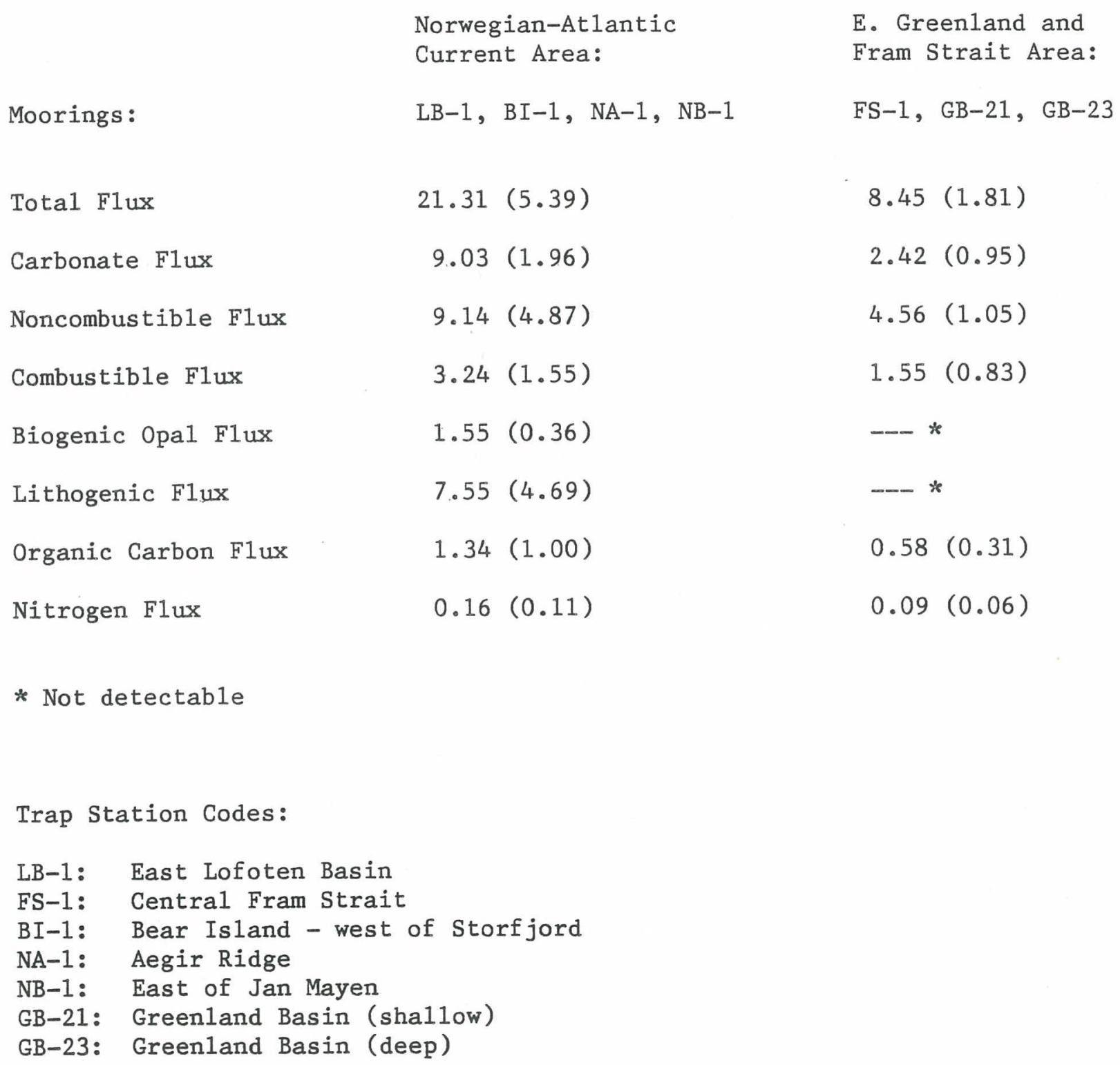


Table 3. Comparison of mass fluxes between 6 stations in the Northern Nordic Seas, 1983-1986.

\begin{tabular}{|c|c|c|c|c|c|c|c|}
\hline Area: & \multicolumn{4}{|c|}{ Norwegian-Atlantic Current } & \multicolumn{3}{|c|}{ East Greenland/Fram Strait } \\
\hline Trap Station: & LB-1 & $B I-1$ & $\mathrm{NA}-1$ & $\mathrm{NB}-1$ & FS-1 & GB-21 & GB-23 \\
\hline Latitude & $69^{\circ} 30 \mathrm{~N}$ & $75^{\circ} 51 \mathrm{~N}$ & $65^{\circ} 31 \mathrm{~N}$ & $70^{\circ} 00 \mathrm{~N}$ & $78^{\circ} 52 \mathrm{~N}$ & $74^{\circ} 35 \mathrm{~N}$ & $75^{\circ} 35 \mathrm{~N}$ \\
\hline Longitude & $10^{\circ} \mathrm{OOE}$ & $11^{\circ} 28 \mathrm{E}$ & $00^{\circ} 64 \mathrm{E}$ & $01^{\circ} 58 \mathrm{~W}$ & $01^{\circ} 22 \mathrm{E}$ & $06^{\circ} 43 W$ & $06^{\circ} 43 \mathrm{~W}$ \\
\hline Trap Depth & $2,760 \mathrm{~m}$ & $1,700 \mathrm{~m}$ & $2,630 \mathrm{~m}$ & $2,749 m$ & $2,440 \mathrm{~m}$ & $1,966 \mathrm{~m}$ & $2,871 \mathrm{~m}$ \\
\hline Tota1 Flux** & 22.80 & 28.40 & 17.36 & 16.79 & 7.20 & 8.79 & 10.21 \\
\hline Carbonate Flux & 11.40 & 6.61 & 9.18 & 8.93 & 1.40 & 2.59 & 3.28 \\
\hline Noncombustible Flux & 8.07 & 16.31 & 5.94 & 6.24 & 4.26 & 3.65 & 5.73 \\
\hline Combustible Flux & 3.37 & 5.35 & 2.31 & 1.90 & 0.92 & 2.50 & 1.23 \\
\hline Biogenic 0pa1 Flux & 1.12 & 1.96 & 1.68 & 1.44 & 0.60 & -- & 2.61 \\
\hline Lithogenic Flux & 6.95 & 14.35 & 4.26 & 4.65 & 4.00 & -- & 3.12 \\
\hline Organic Carbon Flux & 1.37 & 2.85 & 0.59 & 0.53 & 0.41 & 0.94 & 0.40 \\
\hline Nitrogen Flux & 0.18 & 0.30 & 0.08 & 0.08 & 0.06 & 0.16 & 0.06 \\
\hline
\end{tabular}

** Flux is in $\mathrm{mg} \mathrm{m}^{2}$ day

Trap Station Codes:

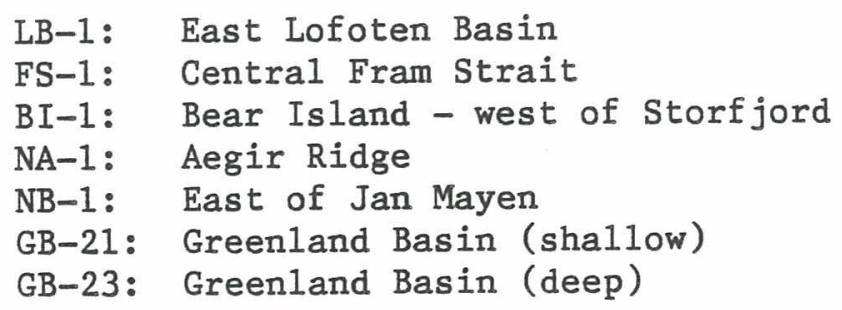




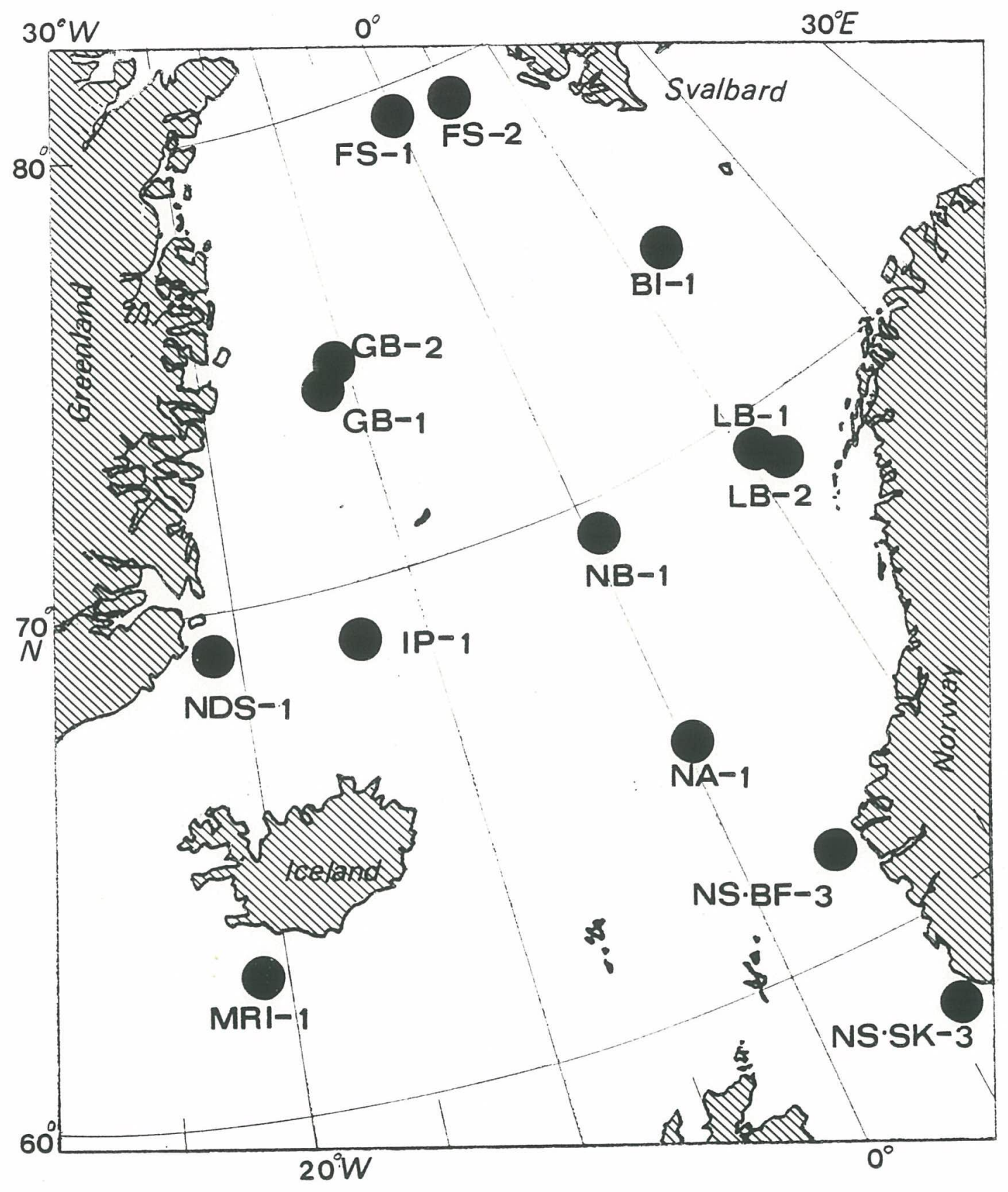

Figure 1. Approximate positions of sediment trap-current meter moorings in the Nordic Seas, 1983-1987. 


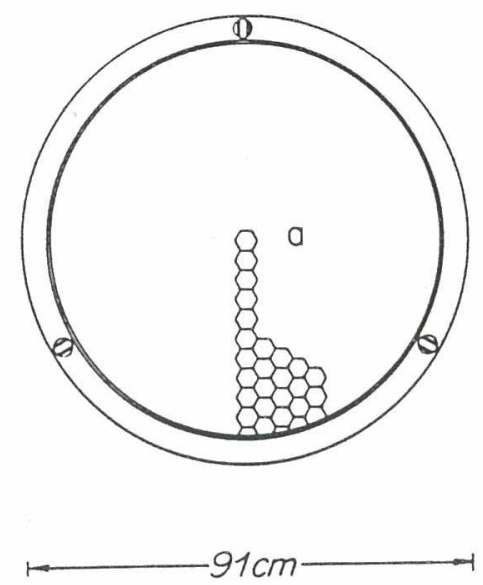

Mark 6-13 $\left(0.5 \mathrm{~m}^{2}\right.$ aperture with 13 sampling bottles).

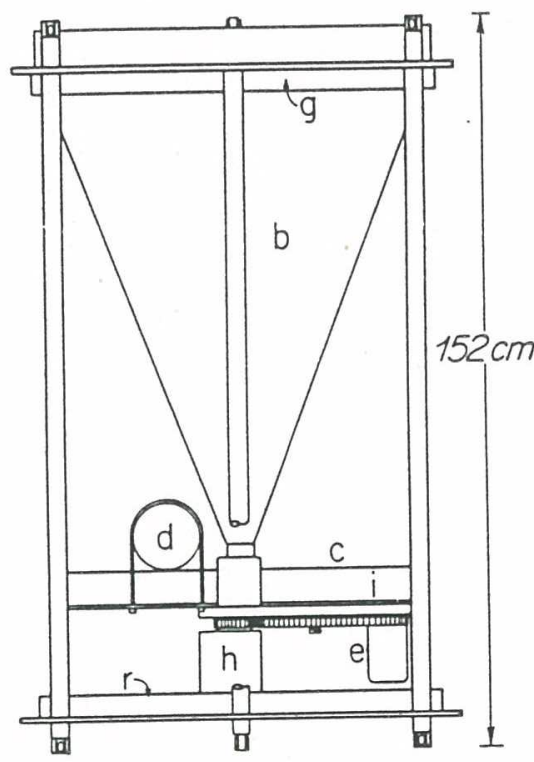

Figure 2. PARFLUX Mark 5 and 6 sediment traps (from Honjo and Doherty, 1987, Fig. 2).

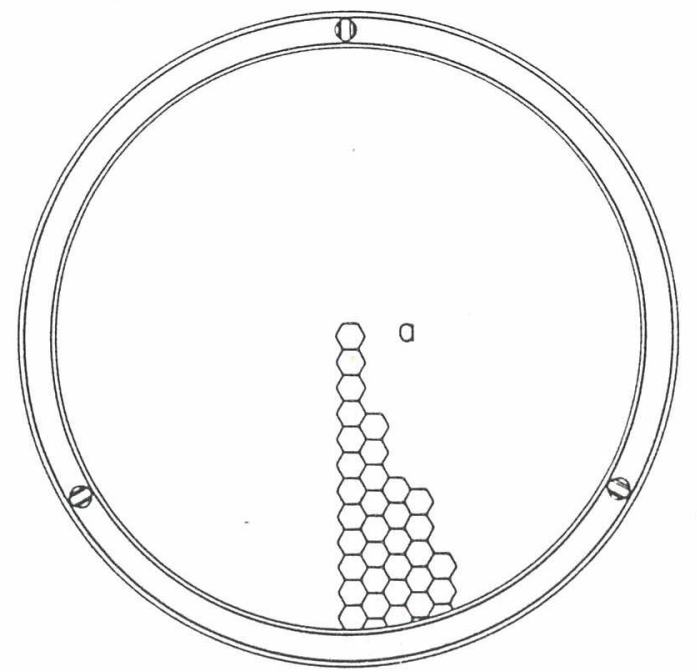

$-135 \mathrm{~cm}$

Mark 5-12 (1.2 $\mathrm{m}^{2}$ aperture with 12 sampling bottles).

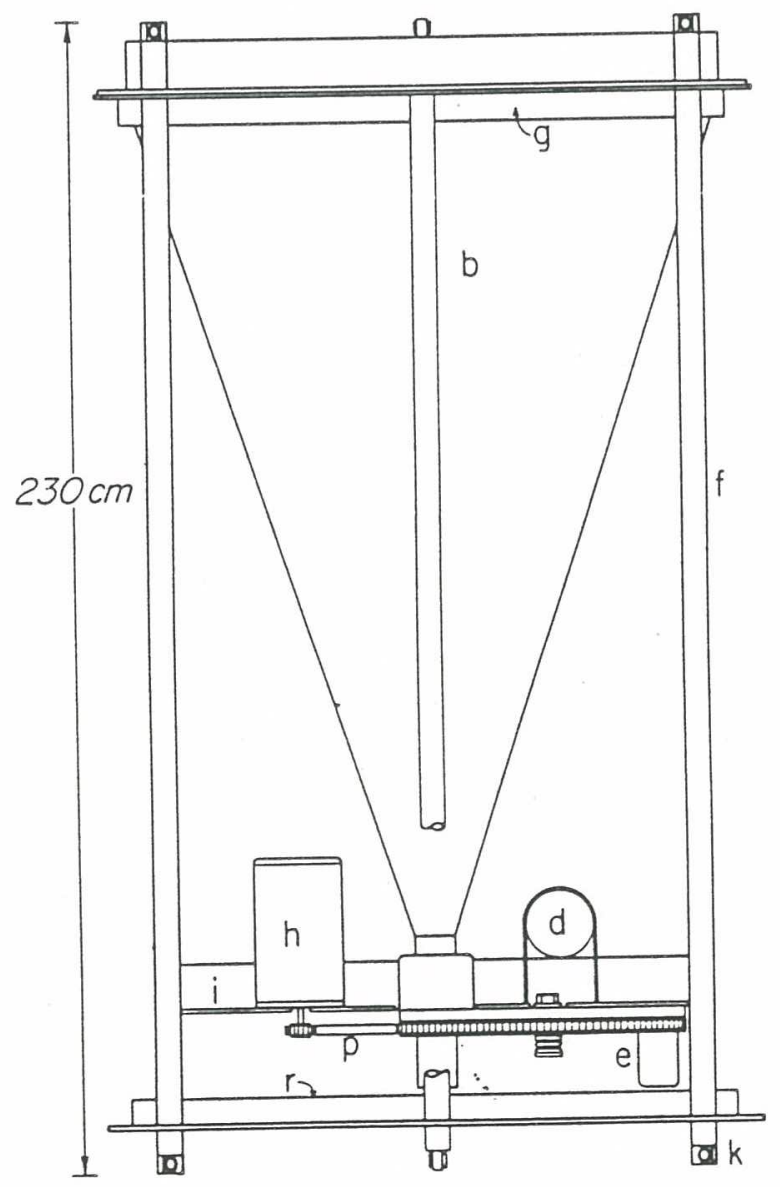




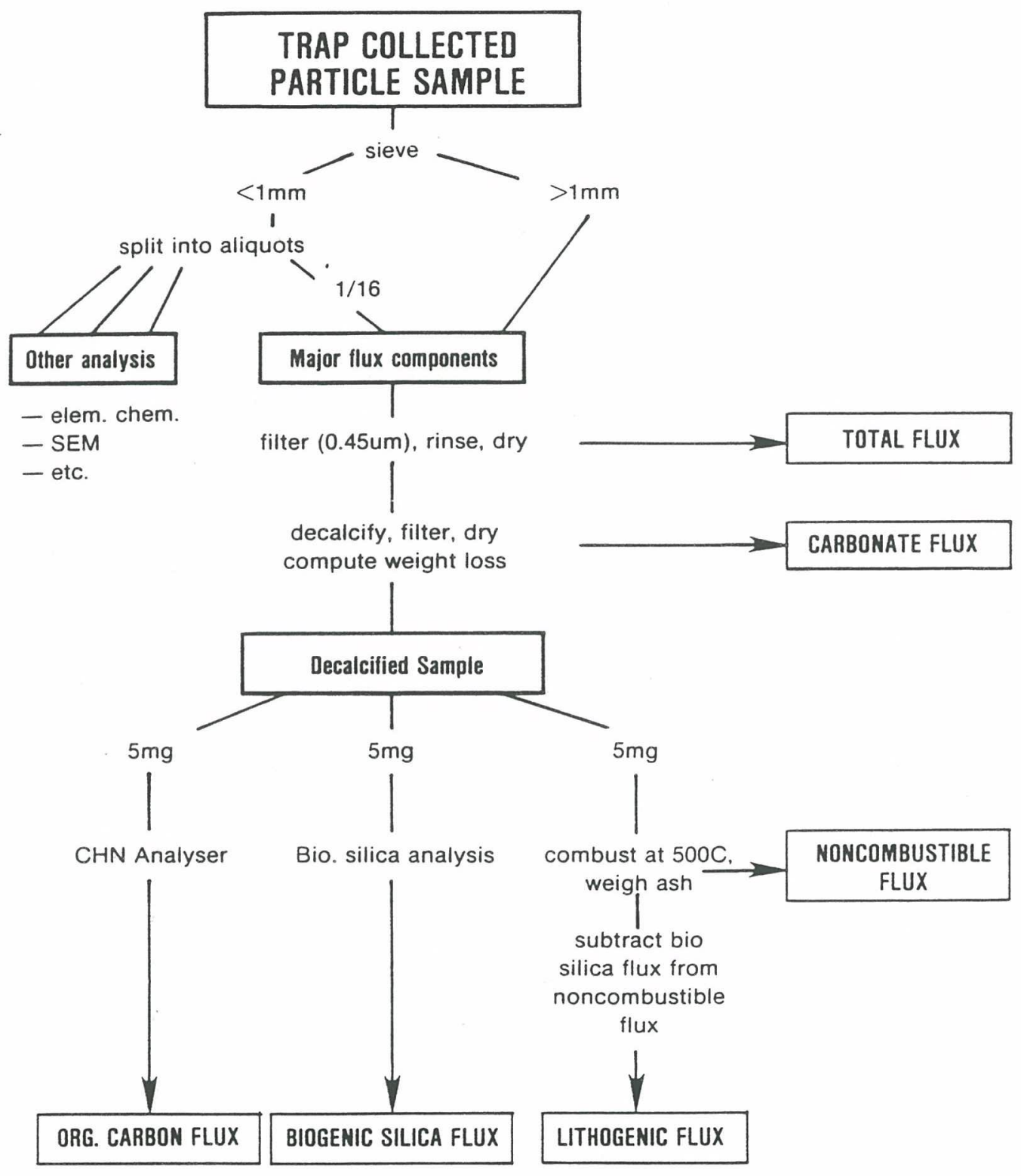

Figure 3. Sample process flow diagram for sedimentological analysis of Nordic Sea flux samples. 
NORWEGIAN-ATLANTIC CURRENT AREA

$-15-$ 


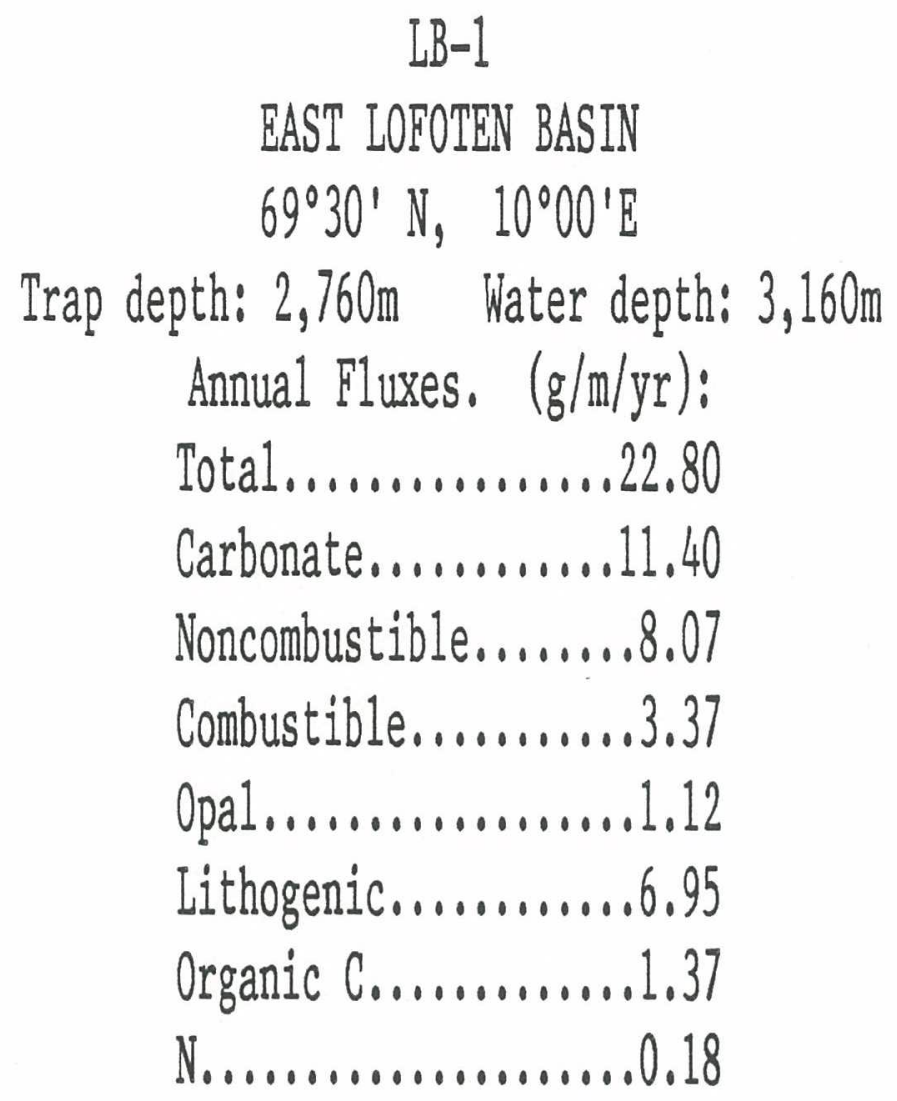

FARFLUX Mark 5-12

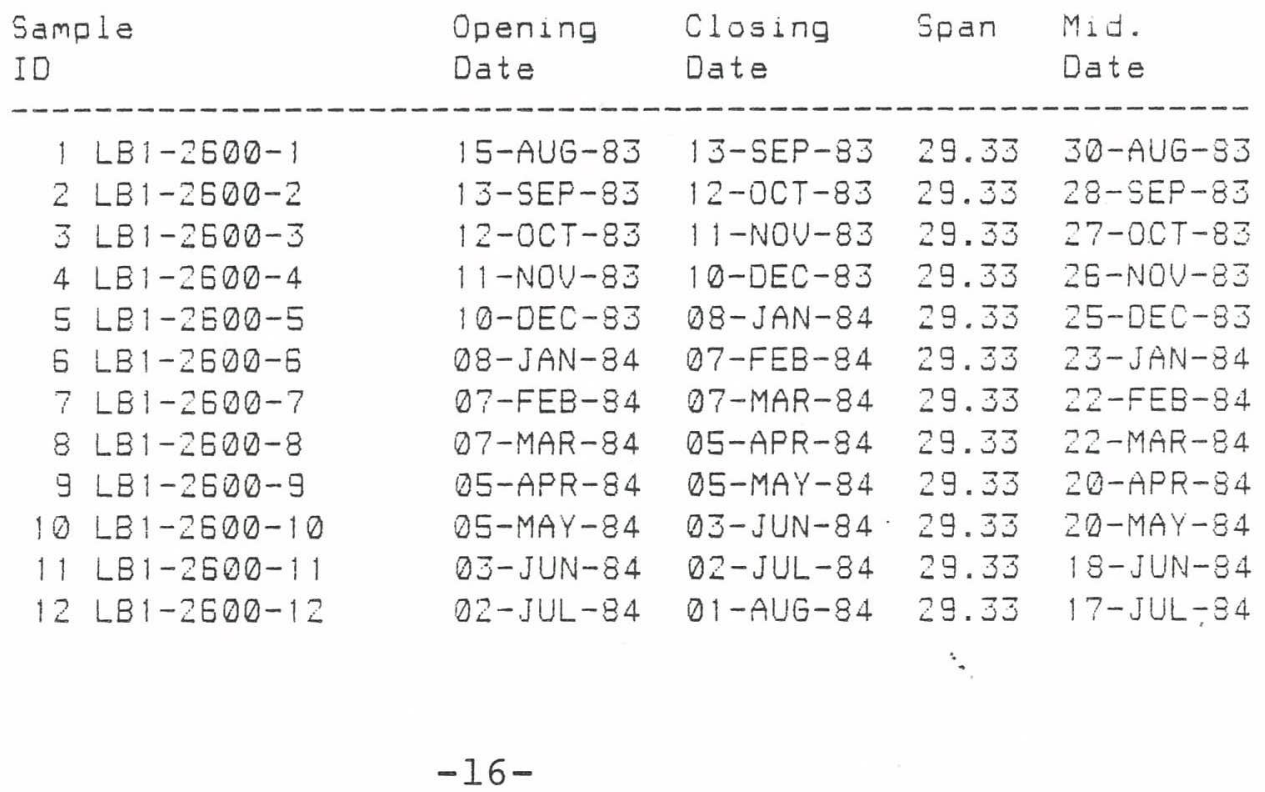


Total FIux at Lofoten Basin (LB-1). 2600m. 1983-1984

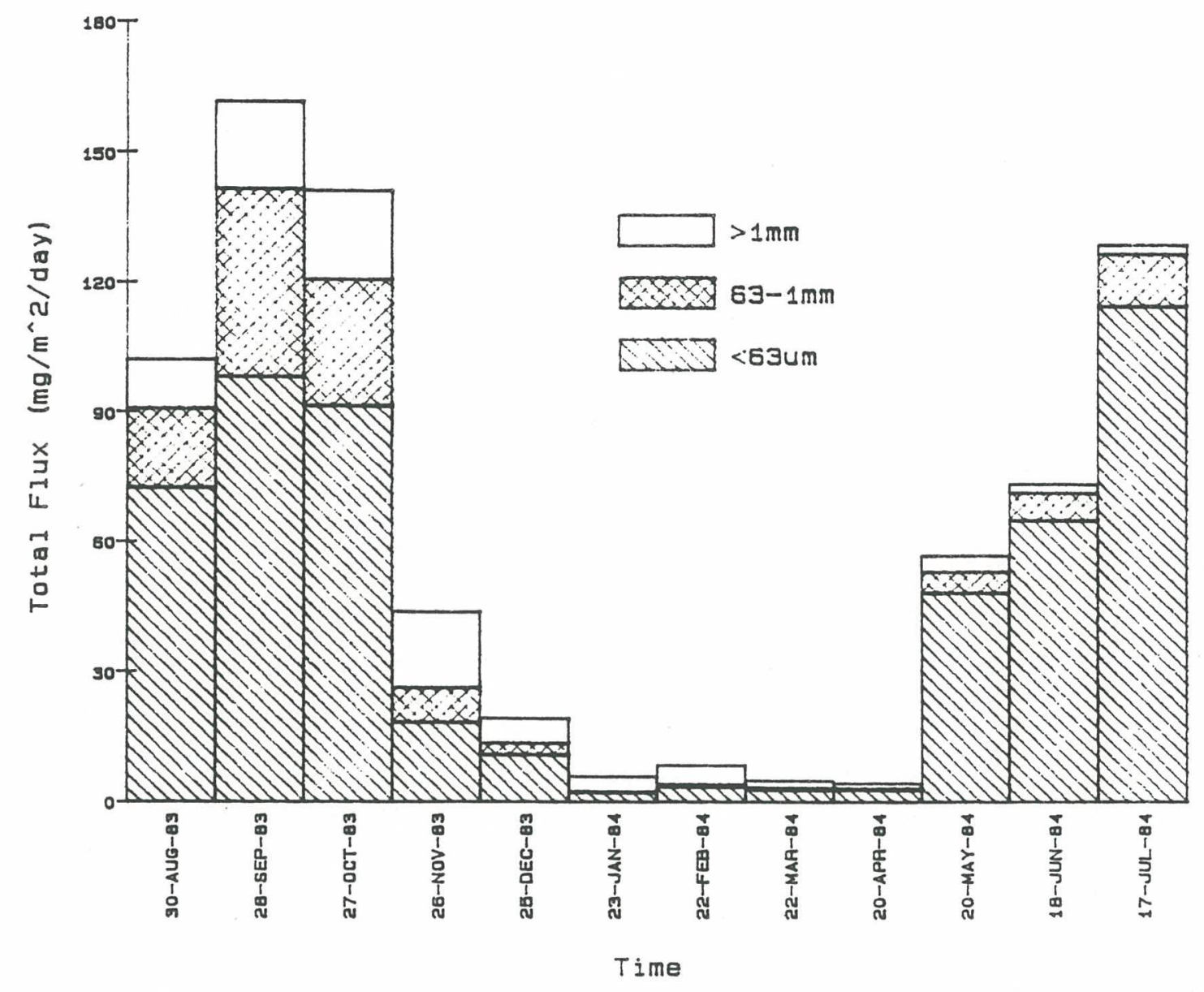

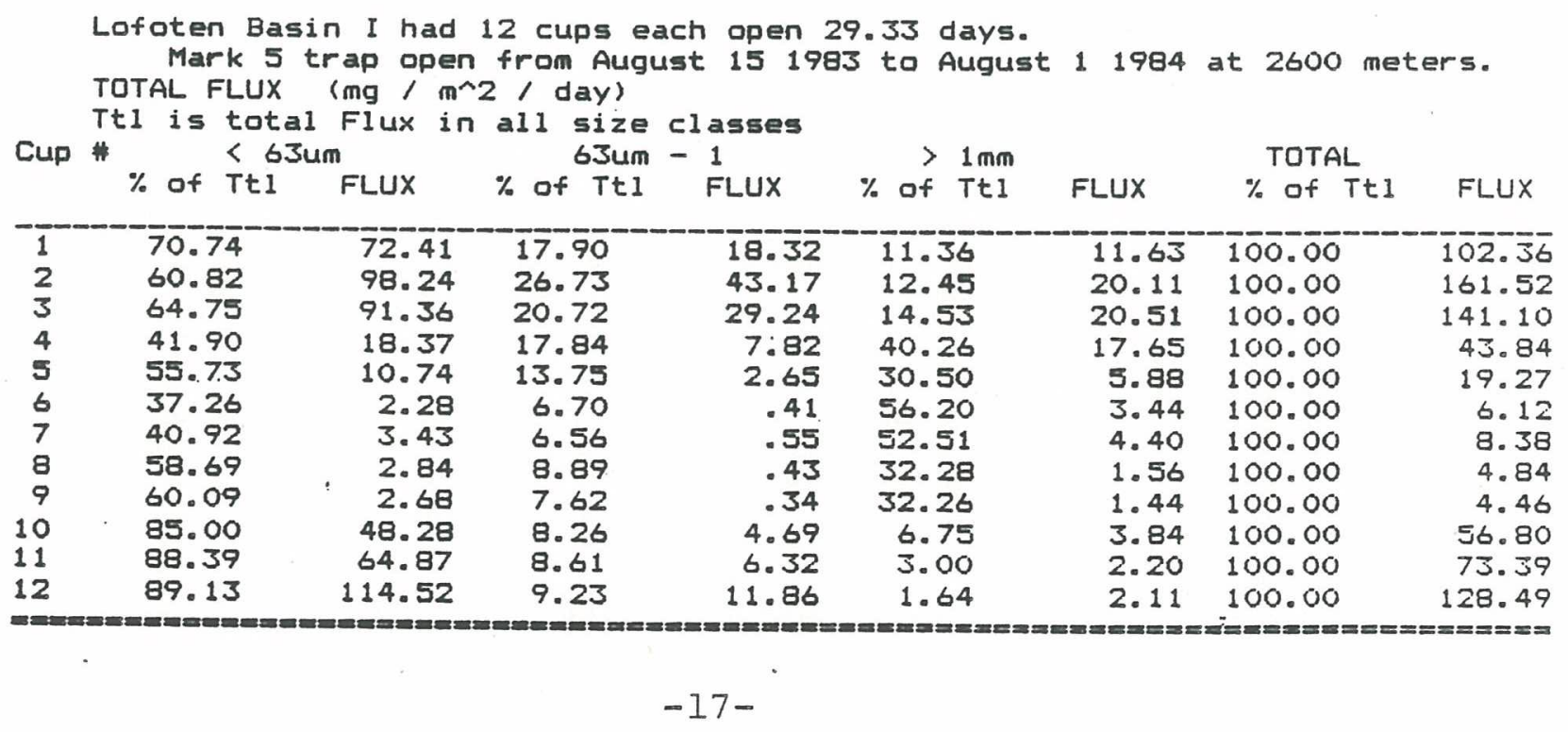


Carbonate Flux at Lofoten Basin (LB-1), 2600m, 1983-1984

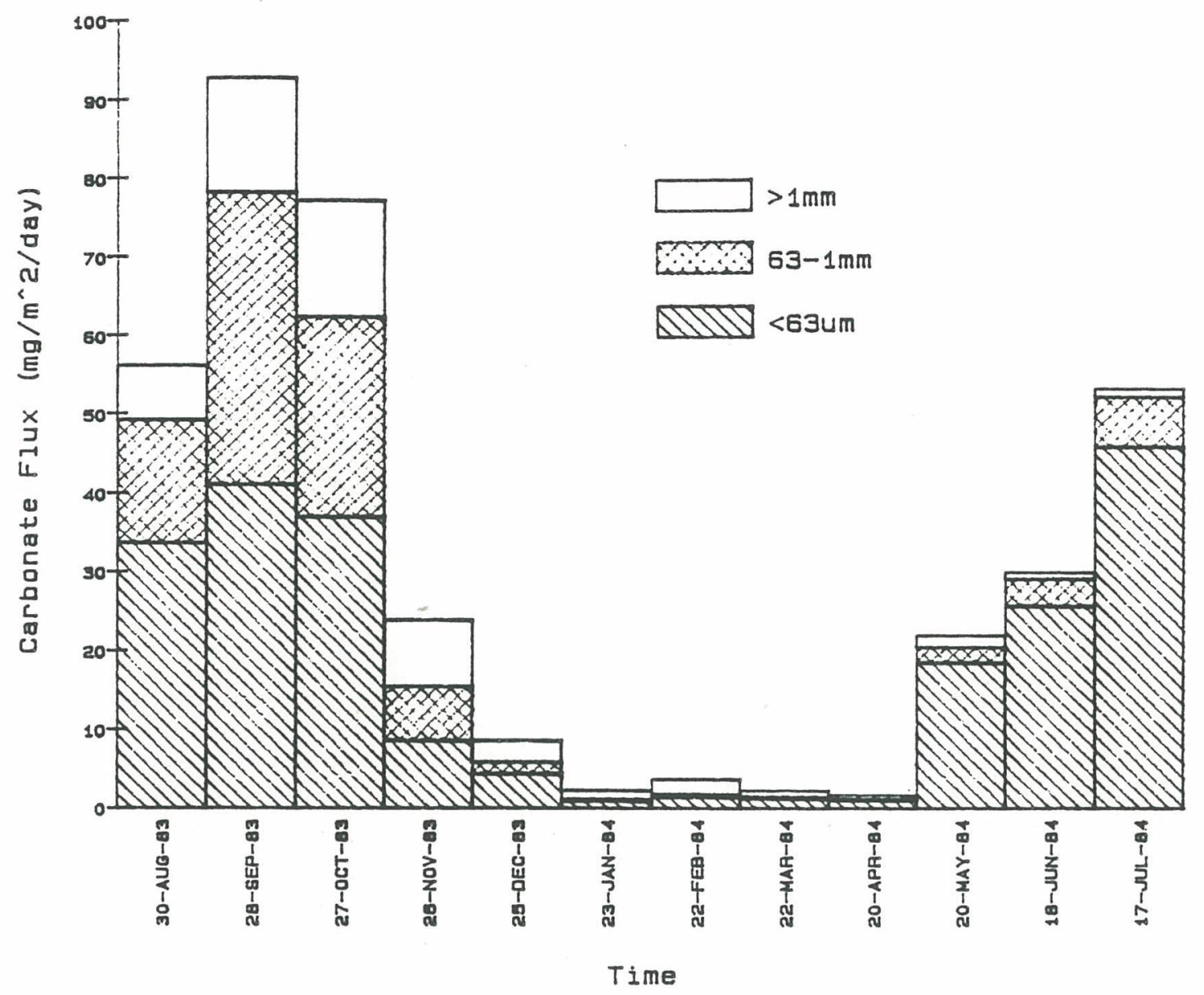

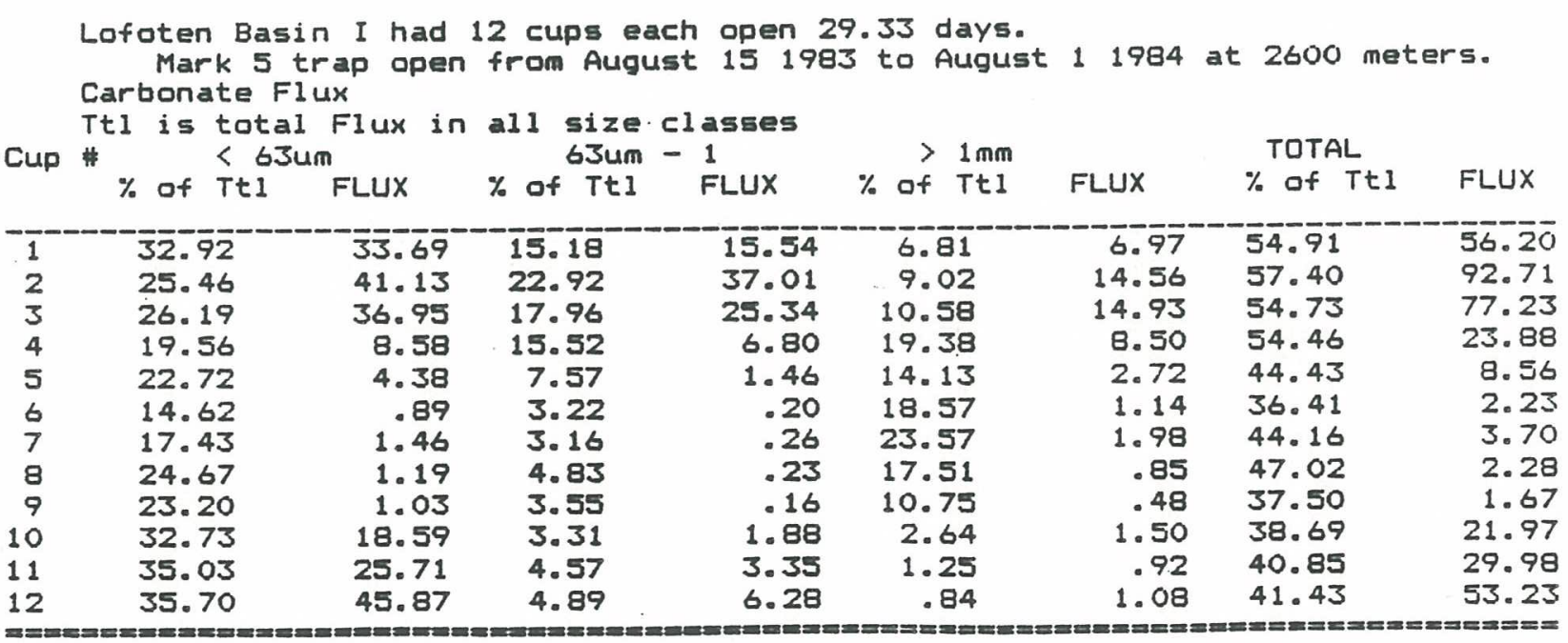


Noncombustible Flux at Lofoten Basin (LB-1). 2600 m. 1983-84

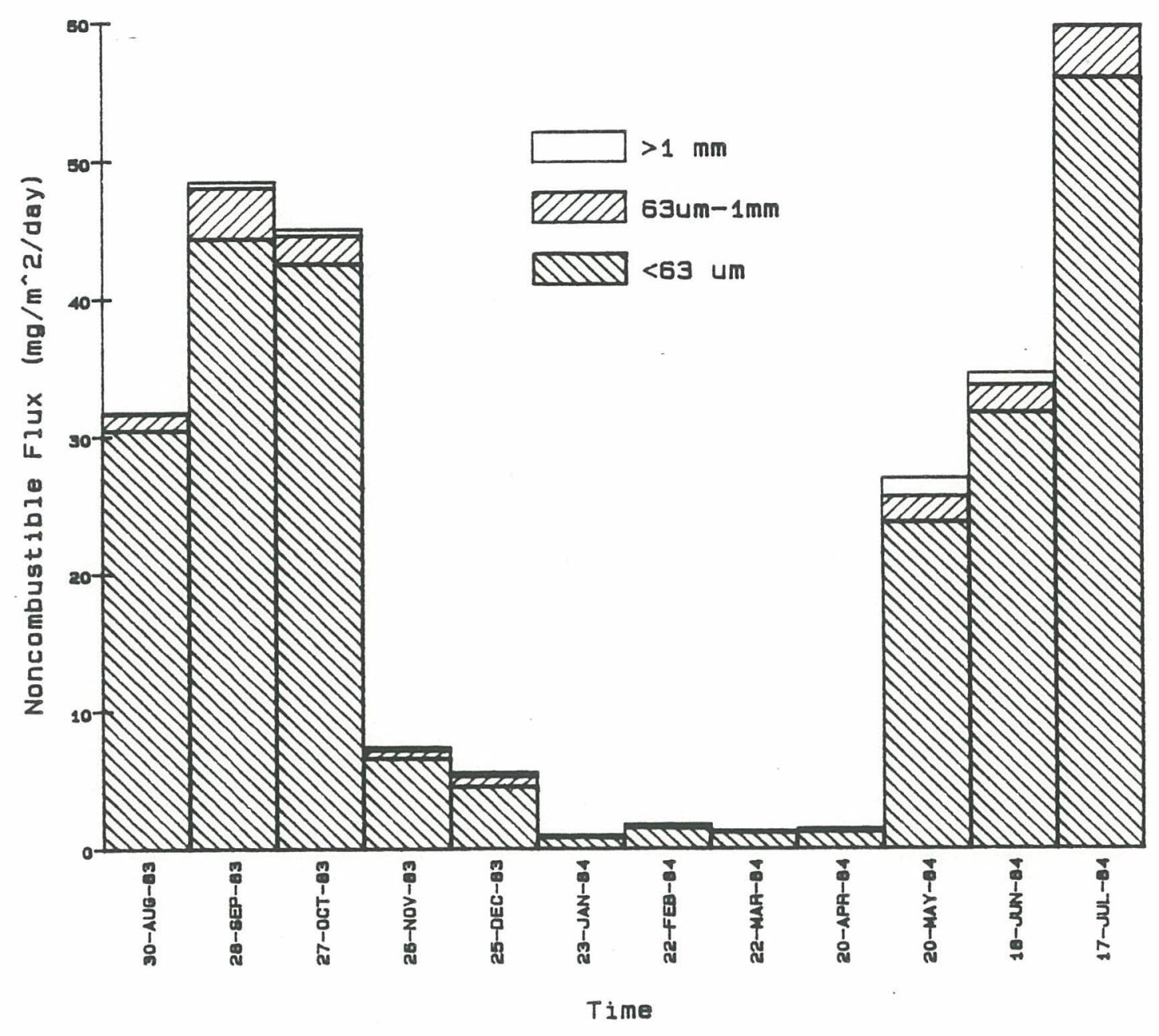

Lofoten Basin I had 12 eups each open 29.33 days.

Mark 5 trap open from Augugt 151983 to August 11984 at 2600 meters. NON COMBUSTIBLE FLUX ( $\mathrm{mg} / \mathrm{m}^{\wedge} \mathrm{z} /$ day)

Ttl is total Flux in all size classes

\begin{tabular}{|c|c|c|c|c|c|c|c|c|}
\hline 1 & 29.76 & 30.46 & 1.75 & 1.18 & 14 & .15 & 31.06 & $31 . \overline{9}$ \\
\hline 2 & 27.47 & 44.37 & 2.29 & 3.69 & .30 & .48 & 30.05 & 48.54 \\
\hline 3 & 30.15 & 42.55 & 1.45 & 2.05 & .32 & .45 & 31.93 & 45.05 \\
\hline 4 & 15.00 & 6.57 & 1.35 & .59 & .58 & .26 & 16.93 & 7.4 \\
\hline 5 & 23.62 & 4.55 & 3.72 & .72 & 1.78 & .34 & 29.12 & 5.6 \\
\hline 6 & 14.30 & .87 & 2.03 & .12 & .83 & .05 & 17.16 & 1.0 \\
\hline 7 & 18.51 & 1.55 & 2.14 & .18 & .44 & .04 & 21.10 & 1.7 \\
\hline 8 & 24.04 & 1.16 & 2.63 & .13 & .00 & .00 & 26.68 & 1.2 \\
\hline 9 & 27.97 & 1.25 & 2.11 & .09 & 4.28 & .19 & 34.36 & 1.5 \\
\hline 10 & 41.79 & 23.74 & 3.33 & 1.89 & 2.44 & 1.38 & 47.55 & 27.0 \\
\hline 11 & 43.15 & 31.66 & 2.67 & 1.96 & 1.28 & .94 & 47.10 & 34.5 \\
\hline 12 & 43.49 & 55.88 & 2.89 & 3.71 & .05 & .06 & 46.43 & 59.6 \\
\hline
\end{tabular}


Combustible Flux at Lofoten Basin (LB-1), $2600 \mathrm{~m}, 1983-84$

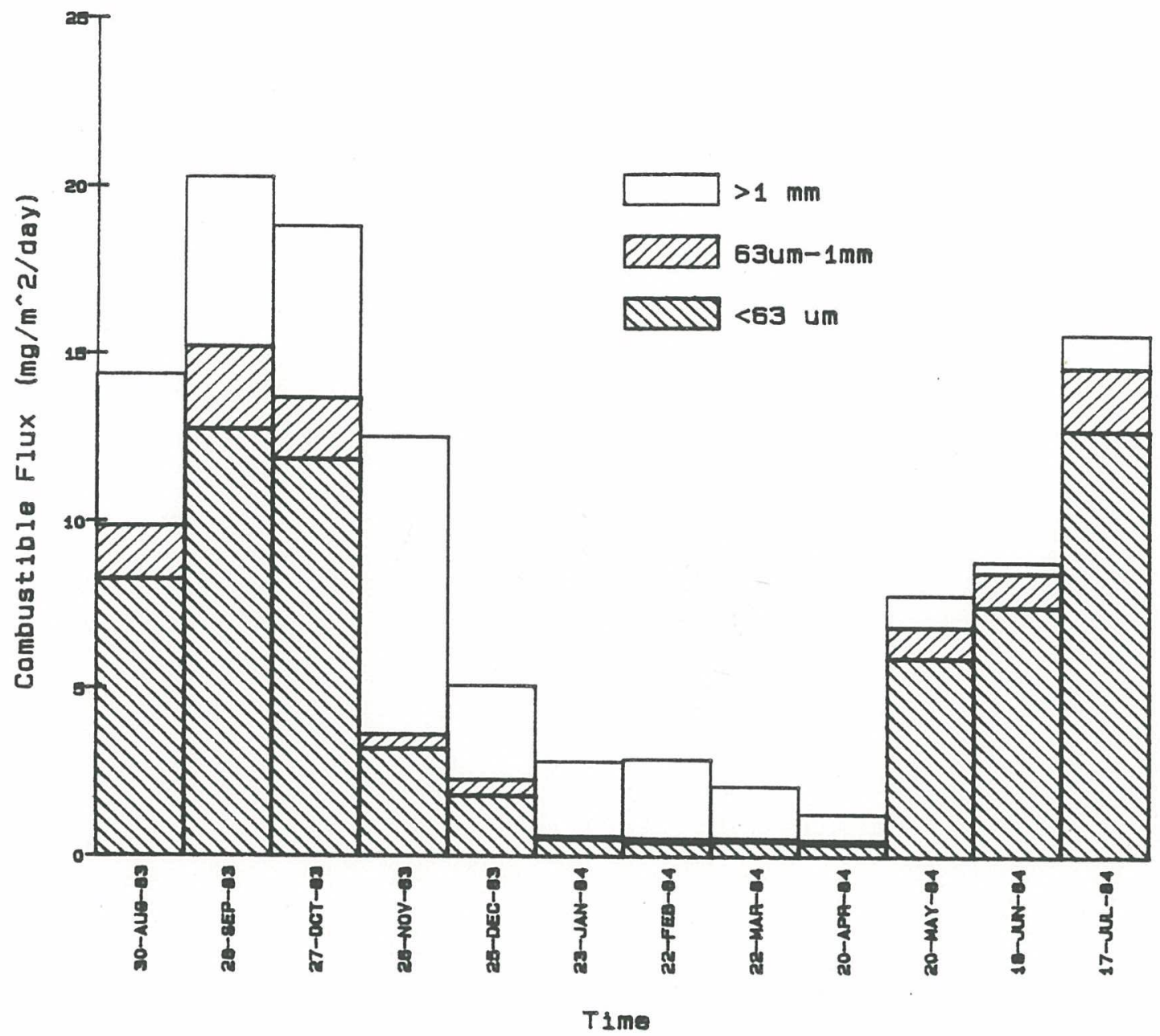

Lofoten Basin I had 12 cups each open 29.33 days.

Mark 5 trap open from August 151983 to Auqust 11984 at 2600 meters. Combustible Flux

Ttl is total Flux in all gize classes

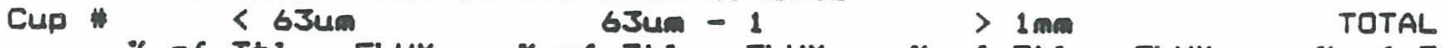

$\%$ of Tt1 FLUX $\%$ of Tt1 FLUX \% of Tt1 FLUX \% of Tt1 FLUX

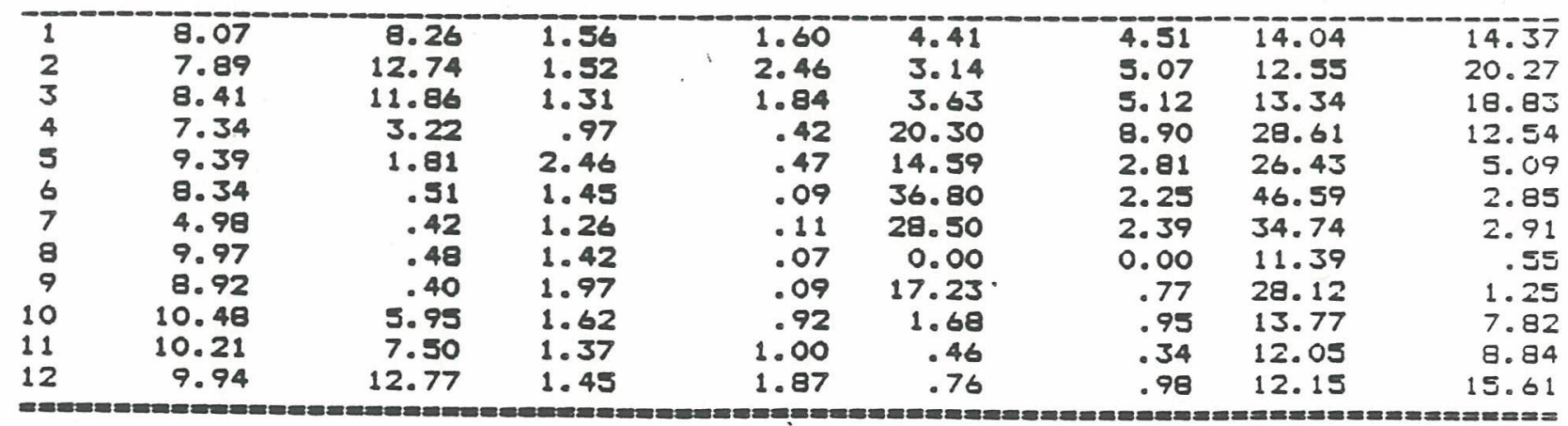


Biogenic Silica flux at Lofoten Basin (LB-1). 2600m, 1983-1984

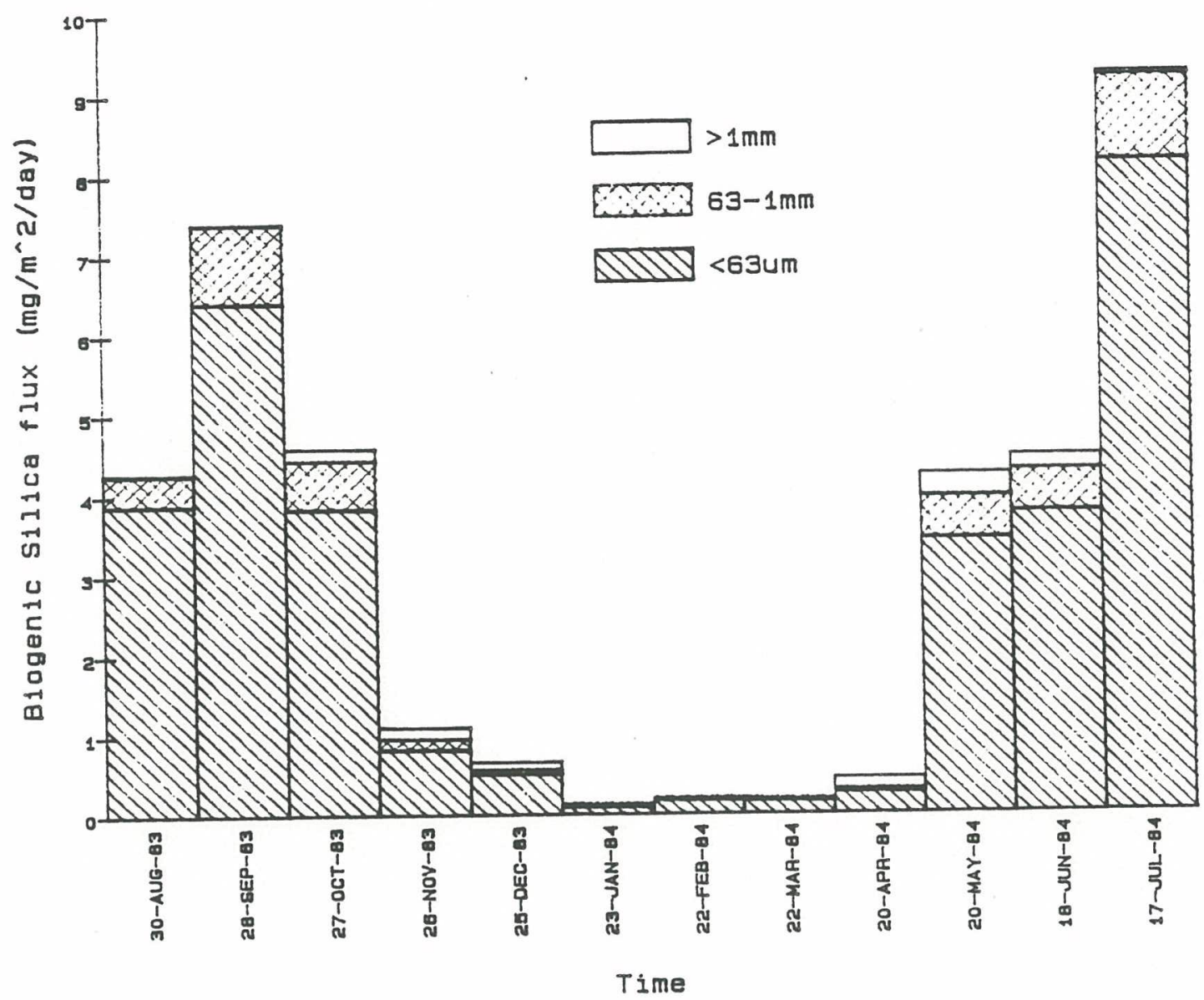

\begin{tabular}{|c|c|c|c|c|c|c|c|c|}
\hline $\begin{array}{l}\text { Sample } \\
\text { ID\# }\end{array}$ & $\begin{array}{l}\text { OPAL } \\
63\end{array}$ & $\begin{array}{l}\text { OPAL } \% \\
\text { tot. }<63\end{array}$ & $\begin{array}{l}\text { OPAL } \\
63-1\end{array}$ & $\begin{array}{l}\text { OPAL } \% \\
\text { tot. } 63-1\end{array}$ & $\begin{array}{l}O P A L \\
>1\end{array}$ & $\begin{array}{l}\text { OPAL } \% \\
\text { tot. }>1\end{array}$ & $\begin{array}{l}\text { OPAL } \\
\text { total }\end{array}$ & $\begin{array}{l}\text { OPAL \% } \\
\text { of total }\end{array}$ \\
\hline 1 LBI-2E00-1 & 3.87 & 3.78 & 0.38 & 0.37 & 0.03 & 0.03 & 4.28 & 4.18 \\
\hline $2 \angle B 1-2 E 00-2$ & 6.40 & 3.96 & 0.99 & 0.61 & 0.01 & 0.01 & 7.40 & 4.58 \\
\hline $3 \angle B 1-2600-3$ & 3.82 & 2.71 & $0 . E 0$ & 0.43 & 0.16 & 0.11 & 4.57 & 3.24 \\
\hline $4 L B 1-2600-4$ & 0.82 & 1.87 & 0.13 & 0.30 & 0.15 & 0.35 & 1.11 & 2.53 \\
\hline 5 LB $1-2 E 00-5$ & 0.51 & 2.65 & 0.05 & 0.26 & 0.10 & 0.54 & $0.6 E$ & 3.45 \\
\hline $6 L E 1-2600-6 *$ & 0.09 & 1.47 & 0.09 & 0.02 & 0.00 & 0.03 & 0.09 & $: .47$ \\
\hline 7 LB $1-2600-7$ & 0.17 & 2.03 & 0.03 & 0.32 & 0.19 & 2.27 & 0.39 & 4.55 \\
\hline $8 L B 1-2600-8 *$ & 0.15 & 2.61 & 0.25 & 0.64 & 0.06 & 1.23 & 0.25 & $5 .: 7$ \\
\hline $9 L B 1-2600-9$. & $0.2 E$ & 4.25 & 0.25 & 0.67 & 0.15 & 3.31 & 0.44 & 9.87 \\
\hline 10 LB1-2600-10 & 3.42 & 6.02 & 0.53 & 0.93 & 0.30 & 0.53 & 4.25 & 7.47 \\
\hline 11 LB1-2600-11 & 3.75 & 5.11 & 0.52 & 0.71 & 0.18 & 0.24 & 4.45 & 5.00 \\
\hline $12 L B 1-2600-12$ & 8.12 & 6.32 & 1.04 & 0.81 & 0.05 & 0.04 & 9.21 & .17 \\
\hline
\end{tabular}

Flux is in mg/m"z/day. 


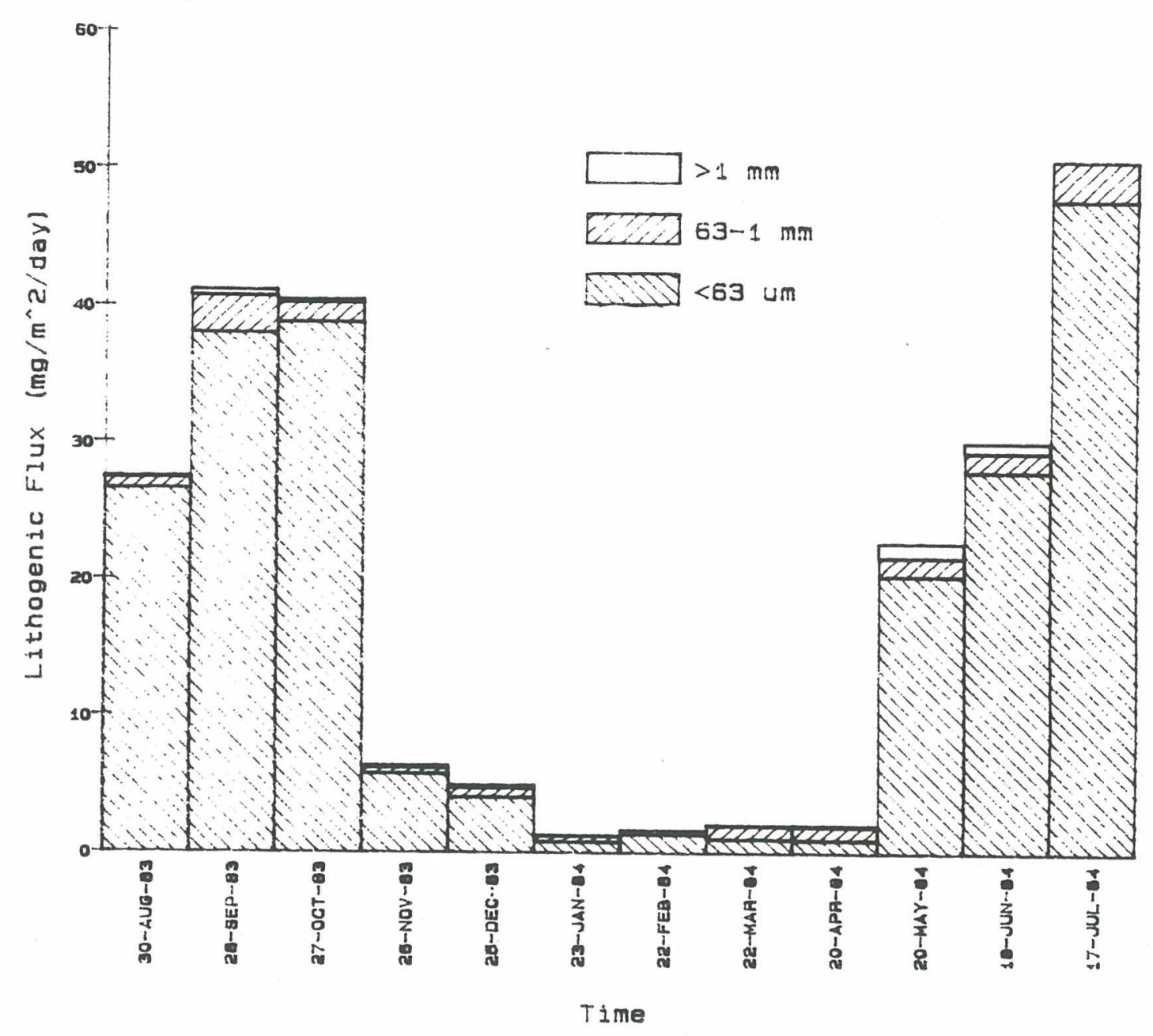

\begin{tabular}{|c|c|c|c|c|c|c|c|c|}
\hline $\begin{array}{l}\text { Sample } \\
\text { I.D. }\end{array}$ & $\begin{array}{l}\mathrm{LITH} \\
\mathbf{6} 33 \\
\end{array}$ & $\begin{array}{l}\text { LITH }<63 \\
\text { \%tot. }\end{array}$ & $\begin{array}{l}\text { LITH } \\
63-1\end{array}$ & $\begin{array}{l}\text { LITHE3-1 } \\
\text { \%tot. }\end{array}$ & $\operatorname{LITH}_{1}$ & $\begin{array}{l}\text { LITH>1 } \\
\text { \%tot. }\end{array}$ & $\begin{array}{l}\text { LITH } \\
\text { total }\end{array}$ & $\begin{array}{r}\text { LITH } \% \\
\text { total }\end{array}$ \\
\hline 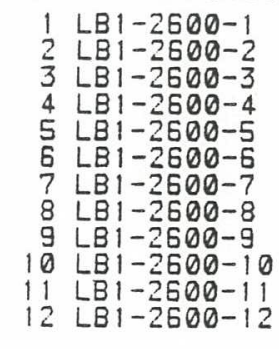 & $\begin{array}{r}25.59 \\
37.97 \\
38.73 \\
5.75 \\
4.04 \\
0.78 \\
1.38 \\
1.01 \\
0.99 \\
20.31 \\
27.91 \\
47.76\end{array}$ & $\begin{array}{l}25.98 \\
23.51 \\
27.45 \\
13.11 \\
20.96 \\
12.75 \\
16.46 \\
20.87 \\
22.20 \\
35.76 \\
38.03 \\
37.17\end{array}$ & $\begin{array}{l}0.80 \\
2.71 \\
1.45 \\
0.46 \\
0.67 \\
0.43 \\
0.18 \\
0.99 \\
0.99 \\
1.36 \\
1.43 \\
2.87\end{array}$ & $\begin{array}{r}0.78 \\
1.68 \\
1.03 \\
1.05 \\
3.48 \\
7.03 \\
2.15 \\
20.45 \\
22.20 \\
2.39 \\
1.95 \\
2.23\end{array}$ & $\begin{array}{l}0.12 \\
0.47 \\
0.29 \\
0.11 \\
0.24 \\
0.05 \\
0.04 \\
0.04 \\
0.04 \\
1.08 \\
0.76 \\
0.01\end{array}$ & $\begin{array}{l}0.12 \\
0.29 \\
0.21 \\
0.25 \\
1.25 \\
0.82 \\
0.48 \\
0.83 \\
0.90 \\
1.90 \\
1.04 \\
0.01\end{array}$ & $\begin{array}{r}27.51 \\
41.15 \\
40.47 \\
6.32 \\
4.95 \\
1.26 \\
1.60 \\
2.04 \\
2.02 \\
22.75 \\
30.10 \\
50.64\end{array}$ & $\begin{array}{l}26.88 \\
25.48 \\
28.68 \\
14.42 \\
25.69 \\
20.59 \\
19.09 \\
42.16 \\
45.29 \\
40.05 \\
41.01 \\
39.41\end{array}$ \\
\hline
\end{tabular}

Flux is in $\mathrm{mg} / \mathrm{m}^{\wedge} 2 /$ day 
Carbon Flux at Lofoten Basin (LB-1). $2600 \mathrm{~m}_{0}$ 1983-84

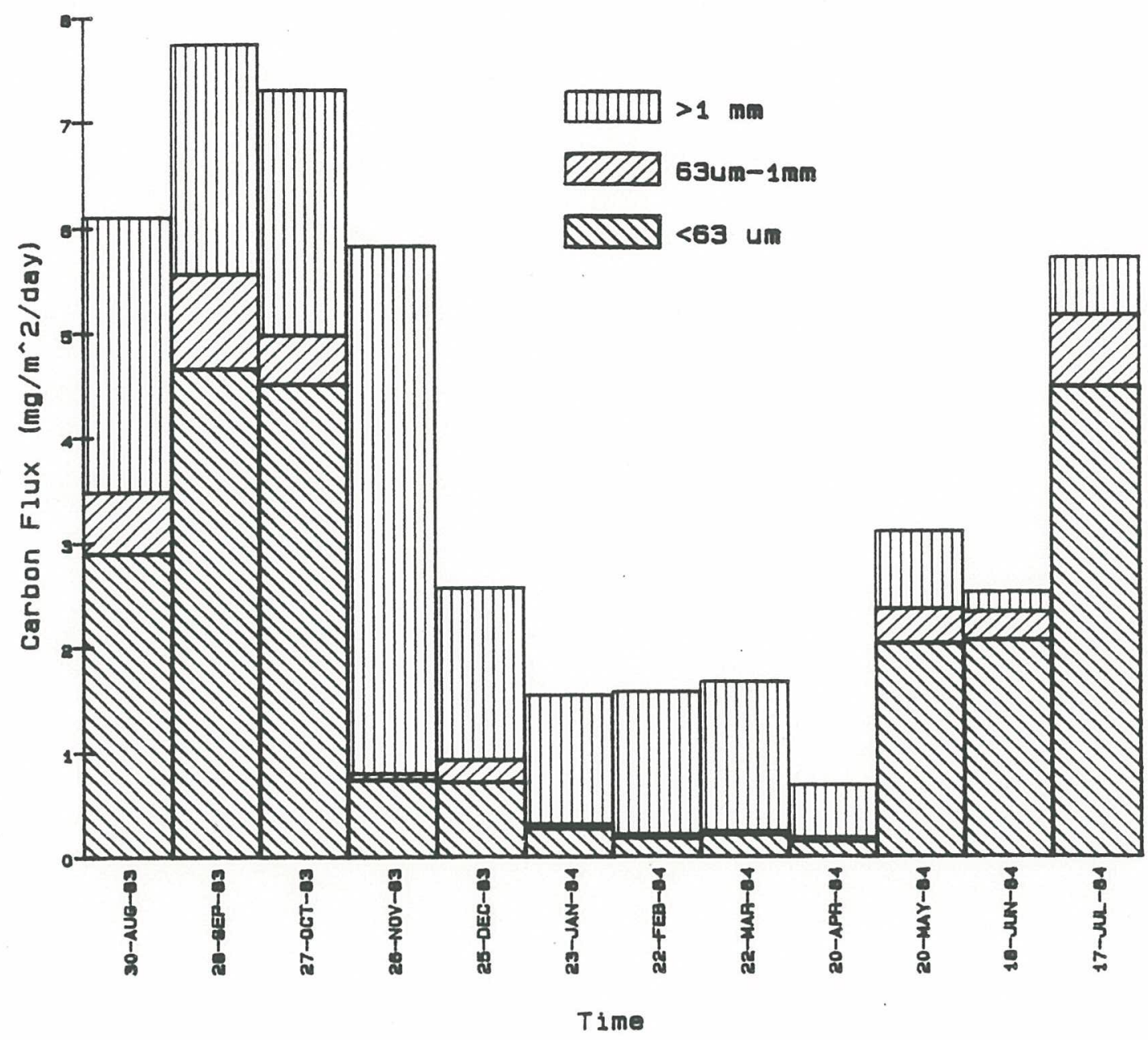

\begin{tabular}{|c|c|c|c|c|c|c|c|c|}
\hline $\begin{array}{l}\text { Sample } \\
\text { I.D. }\end{array}$ & $\begin{array}{l}\text { NTGN } \\
: 63\end{array}$ & $\begin{array}{l}\text { NTGN } 63 \\
\text { \%cmb f. }\end{array}$ & $\begin{array}{l}\text { NTGN } \\
63-1\end{array}$ & $\begin{array}{l}\text { NTGN63-1 } \\
\text { \%cMOF. }\end{array}$ & $\begin{array}{l}\text { NTGN } \\
\vdots 1\end{array}$ & $\begin{array}{l}\text { NTGN: } 1 \\
\text { \%cmb f. }\end{array}$ & $\begin{array}{l}\text { NTGN } \\
\text { total }\end{array}$ & $\begin{array}{l}\text { NTGNtot. } \\
\text { \%embi. }\end{array}$ \\
\hline 1 L81-2600-1 & 0.40 & 4.84 & 0.16 & 1.11 & 0.21 & 1.46 & 0.76 & 5.29 \\
\hline $2 \angle B 1-2600-2$ & 0.76 & 3.75 & 0.19 & 0.94 & 0.36 & 1.78 & 1. & 6.46 \\
\hline $3 \angle B 1-2600-3$ & 0.66 & 3.51 & 0.10 & 0.53 & 0.30 & 1.59 & 1.06 & 5.63 \\
\hline $4\llcorner 81-2600-4$ & 0.11 & 0.88 & 0.01 & 0.08 & 0.44 & 3.51 & 0.56 & $4.4 ?$ \\
\hline 5 LB1-2600-5 & 0.10 & 1.96 & 0.03 & 0.59 & 0.21 & 4.13 & 0.34 & 6.63 \\
\hline 6 LB1-2600-6. & 0.04 & 1.27 & 0.00 & 0.02 & 0.13 & 4.56 & 0.17 & 5.85 \\
\hline 7 ㄴ1 -2600-7. & 0.02 & 0.69 & 0.01 & 0.34 & 0.13 & 4.47 & 0.16 & 5.50 \\
\hline $8 \angle B 1-2600-8=$ & 0.03 & 1.42 & 0.01 & 0.47 & 0.10 & 4.50 & 0.04 & 1.70 \\
\hline $9\llcorner B 1-2600-9$. & 0.02 & 1.60 & 0.00 & 0.00 & 0.06 & 4.80 & 0.08 & $5 . \div 0$ \\
\hline 10 LBI-2600-10 & 0.25 & 3.20 & 0.06 & 0.77 & 0.08 & 1.02 & 0.39 & 4.99 \\
\hline $11 \angle 81-2600-11$ & 0.25 & 2.83 & 0.04 & 0.45 & 0.03 & 0.34 & 0.32 & 3.62 \\
\hline $12 \angle 81-2500-12$ & 0.60 & 3.84 & 0.14 & 0.90 & 0.07 & $0 .+5$ & 0.81 & 5.19 \\
\hline
\end{tabular}

Flux is in mg/m a/day.

"\%cmbf" = "\% of combustable flun". 
Nitrogen Flux at Lofoten Basin (LB-1). $2600 \mathrm{~m}$. 1983-84

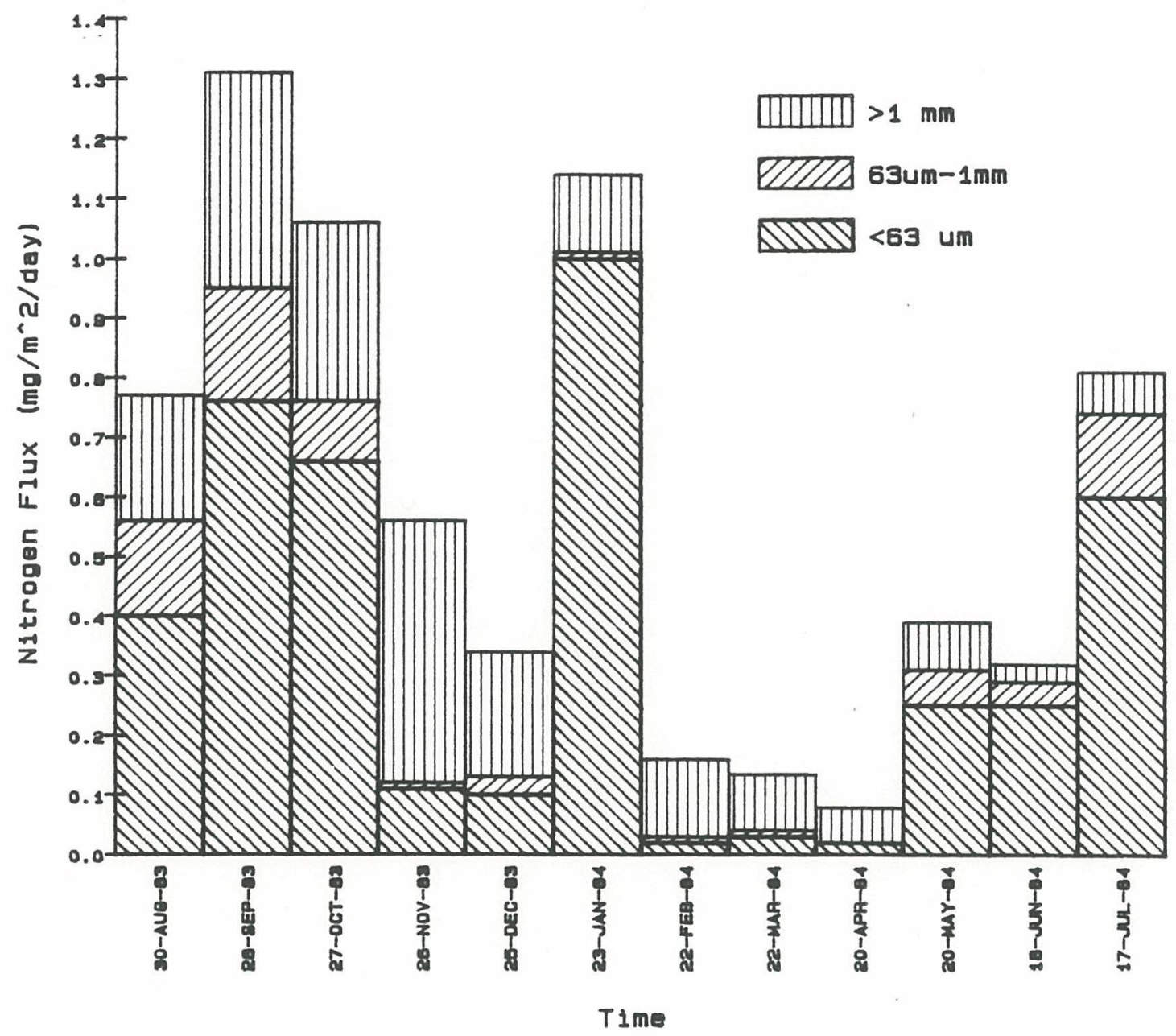

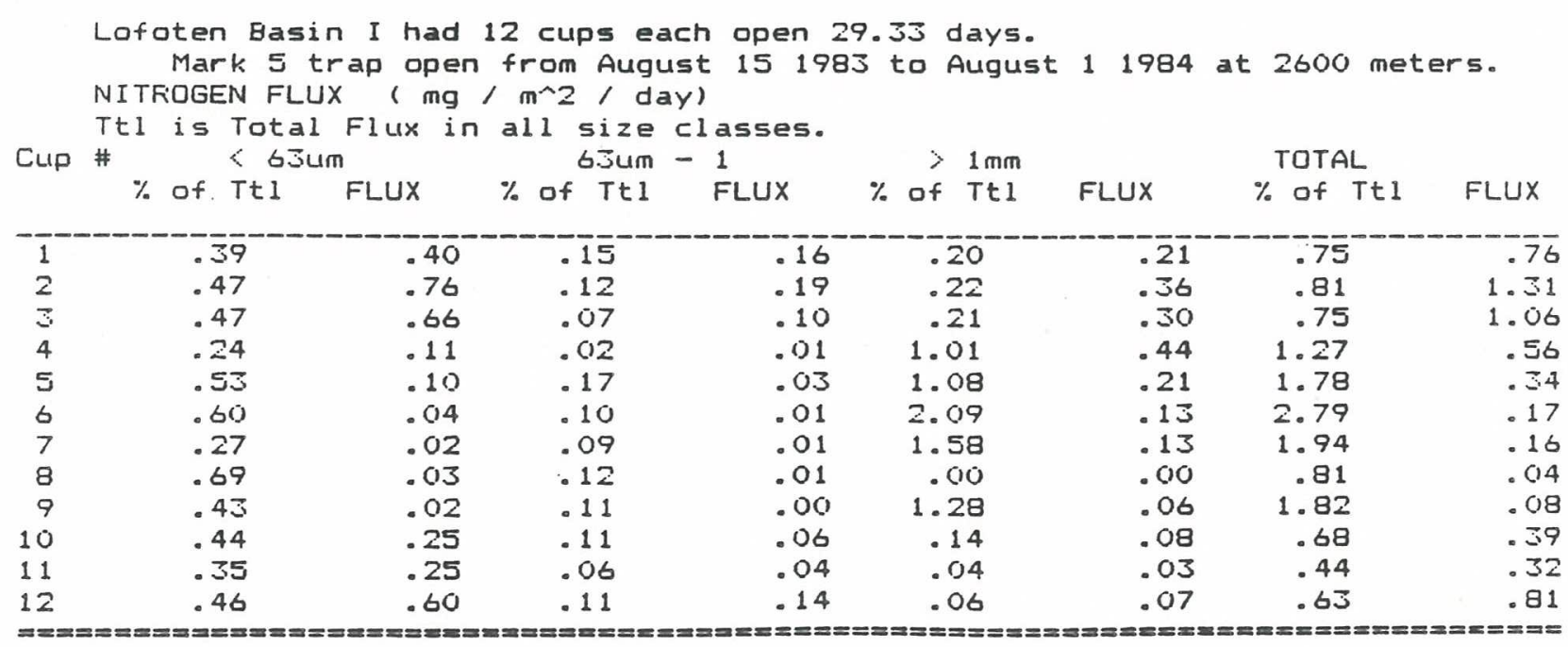


Hydrogen Flux at Lofoten Basin (LB-1), $2600 \mathrm{~m}$. 1983-B4

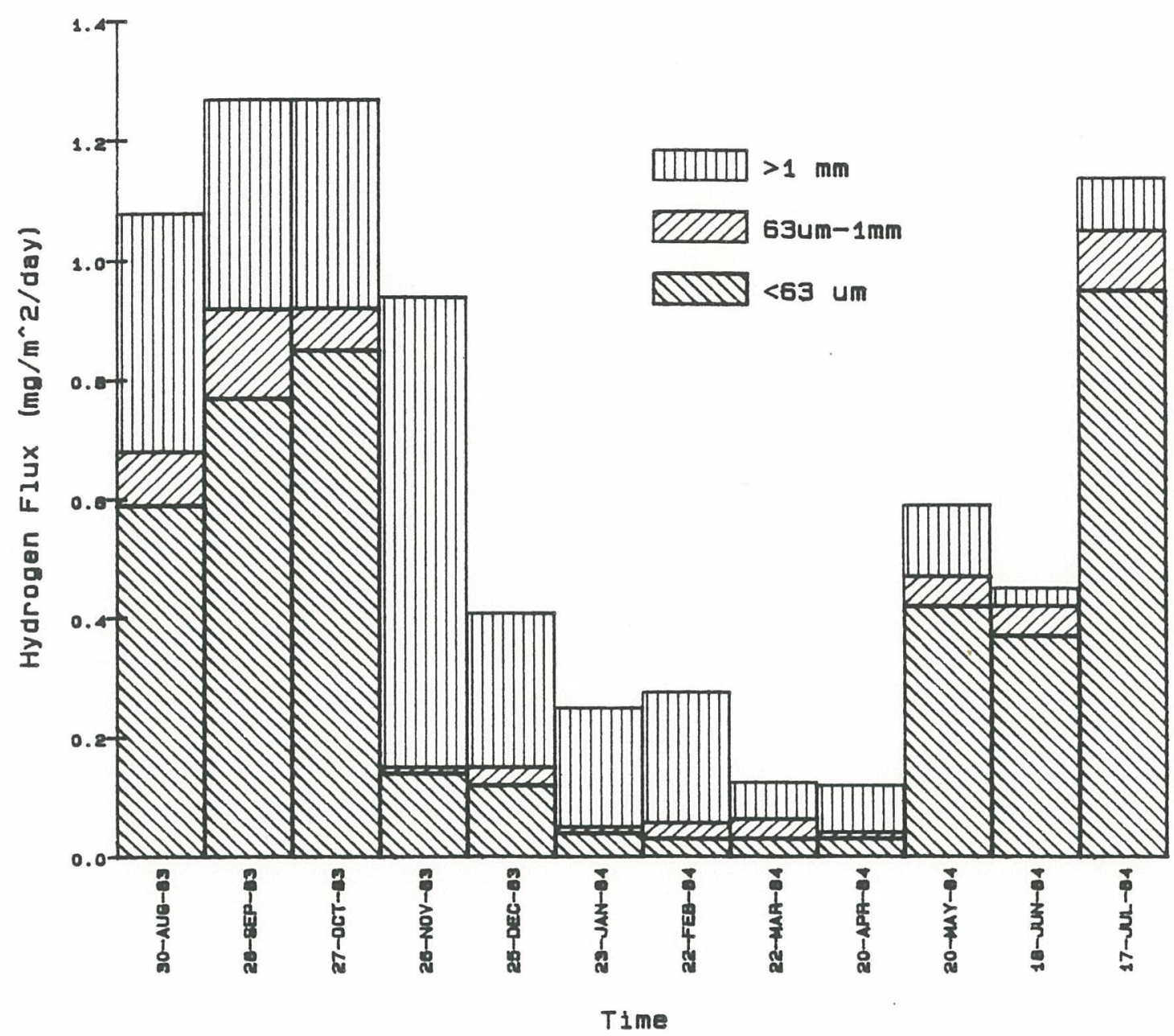

\begin{tabular}{|c|c|c|c|c|c|c|c|c|}
\hline $\begin{array}{l}\text { Sample } \\
\text { I.D. }\end{array}$ & $\begin{array}{l}\text { HYDC } \\
: 63\end{array}$ & $\begin{array}{l}\text { HYDC } \leqslant 63 \\
\text { \%cmb f. }\end{array}$ & $\begin{array}{l}\text { HYDC } \\
63-1\end{array}$ & $\begin{array}{l}\text { HYOCE } 3-1 \\
\% \text { cmb f. }\end{array}$ & $\begin{array}{c}\text { HYOC } \\
1\end{array}$ & $\begin{array}{l}\text { HYOC: } 1 \\
\% \subset M b f .\end{array}$ & $\begin{array}{l}\text { HYOC } \\
\text { total }\end{array}$ & $\begin{array}{l}\text { HYDCtot. } \\
\% \text { EMOf. }\end{array}$ \\
\hline 1 LBI $-2600-1$ & 0.59 & 4.11 & 0.09 & 0.63 & 0.40 & 2.78 & 1.08 & $7.5=$ \\
\hline $2 \angle B \mid-2600-2$ & 0.77 & 3.80 & 0.15 & 0.74 & 0.35 & 1.73 & 1.26 & 6.22 \\
\hline 3 LB $1-2600-3$ & 0.85 & 4.51 & 0.07 & 0.37 & 0.35 & 1.86 & 1.28 & 6.30 \\
\hline $4 L B 1-2600-4$ & 0.14 & 1.12 & 0.01 & 0.08 & 0.79 & 6.30 & 0.94 & 7.50 \\
\hline 5 L81-2500-5 & 0.12 & 2.36 & 0.03 & 0.59 & 0.26 & 5.11 & 0.41 & 8.06 \\
\hline 6 LB $1-2600-6$. & 0.04 & 1.40 & 0.01 & 0.35 & 0.20 & 7.02 & 0.24 & 3.42 \\
\hline 7 LB $1-2600-7$. & 0.03 & 1.03 & 0.03 & 0.92 & 0.22 & 7.56 & 0.25 & 8.59 \\
\hline $8\llcorner B \mid-2600-8$. & 0.03 & 1.42 & 0.03 & 1.56 & 0.06 & 2.93 & 0.04 & 1.90 \\
\hline $9 L B 1-2600-9$. & 0.03 & 2.40 & 0.01 & 0.80 & 0.08 & 6.40 & 0.11 & 8.80 \\
\hline 10 LB $1-2600-10$ & 0.42 & 5.37 & 0.05 & $\because 0.64$ & 0.12 & 1.53 & 0.59 & 7.54 \\
\hline 11 LB1-2600-11 & 0.37 & 4.19 & 0.05 & 0.57 & 0.03 & 0.34 & 0.45 & 5.09 \\
\hline $12 \angle B 1-2600-12$ & 0.95 & 6.09 & 0.10 & 0.64 & 0.09 & 0.58 & 1.15 & 7.37 \\
\hline
\end{tabular}

Flux is in mg/m Z'day.

"\%ombf " $" \%$ of combustible flua". 


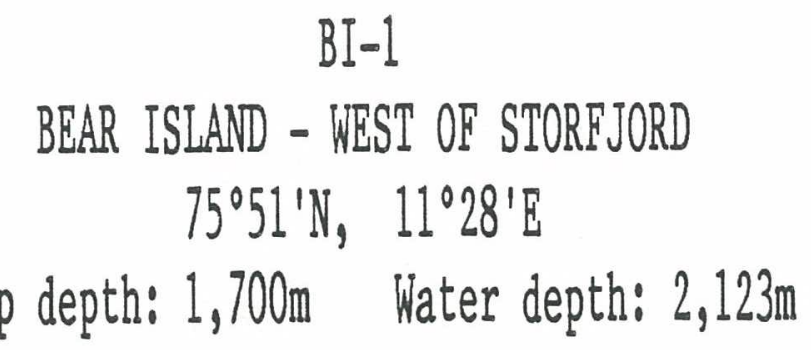

Annual Fluxes. $\left(g / g^{2} / y r\right)$ :

Total.................28.30

Carbonate...............6.61

Noncombustible........16.31

Conbustible............5.38

Biogenic Opa1..........1.96

Lithogenic...........14.35

Organic C..............2.85

No...................... 0.30

PARFLUX Mark 5-13

\begin{tabular}{|c|c|c|c|c|}
\hline $\begin{array}{l}\text { Sample } \\
\text { ID }\end{array}$ & $\begin{array}{l}\text { Opening } \\
\text { Date }\end{array}$ & $\begin{array}{l}\text { Closing } \\
\text { Date }\end{array}$ & Span & $\begin{array}{l}\text { Mid. } \\
\text { Date }\end{array}$ \\
\hline & & & & \\
\hline 26 BI $1-1700-1$ & $12-A \cup G-84$ & $11-5 E P-84$ & 30.17 & $27-A \cup G-84$ \\
\hline 27 BI $1-1700-2$ & $11-S E P-84$ & $11-0 \mathrm{CT}-84$ & 30.17 & $26-5 E P-84$ \\
\hline 28 BI $1-1700-3$ & $11-O C T-84$ & $11-$ NOU-84 & 30.17 & $27-0 \mathrm{CT}-84$ \\
\hline $29 B I 1-1700-4$ & $11-N O U-84$ & $11-D E C-84$ & 30.17 & $26-N O V-84$ \\
\hline 30 BI 1-1700-5 & $11-D E C-84$ & $12-J A N-85$ & 30.17 & $27-D E C-84$ \\
\hline $31 B I 1-1700-6$ & $12-J A N-85$ & $11-F E B-85$ & 30.17 & $27-J A N-85$ \\
\hline 32 BI $1-1700-7$ & $11-F E B-85$ & $12-M A R-85$ & 30.17 & $26-F E B-85$ \\
\hline 33 BI 1-1700-8 & $12-M A R-85$ & $11-A P R-85$ & 30.17 & 27-MAR-85 \\
\hline 34 BI1-1700-9 & $11-A P R-85$ & $12-M A Y-85$ & 30.17 & $27-A P R-85$ \\
\hline 35 BI1-1700-10 & $12-M A Y-85$ & $11-J U N-85$ & 30.17 & $27-M A Y-85$ \\
\hline 36 BI $1-1700-11$ & $11-J U N-85$ & $11-J U L-85$ & 30.17 & $26-J \cup N-85$ \\
\hline 37 BI1-1700-12 & $11-J U L-85$ & $10-A \cup G-85$ & 30.17 & $26-J U L-85$ \\
\hline
\end{tabular}


Total Flux at Bear IsIand ( $I I-1), 1700 m, 1984-1985$

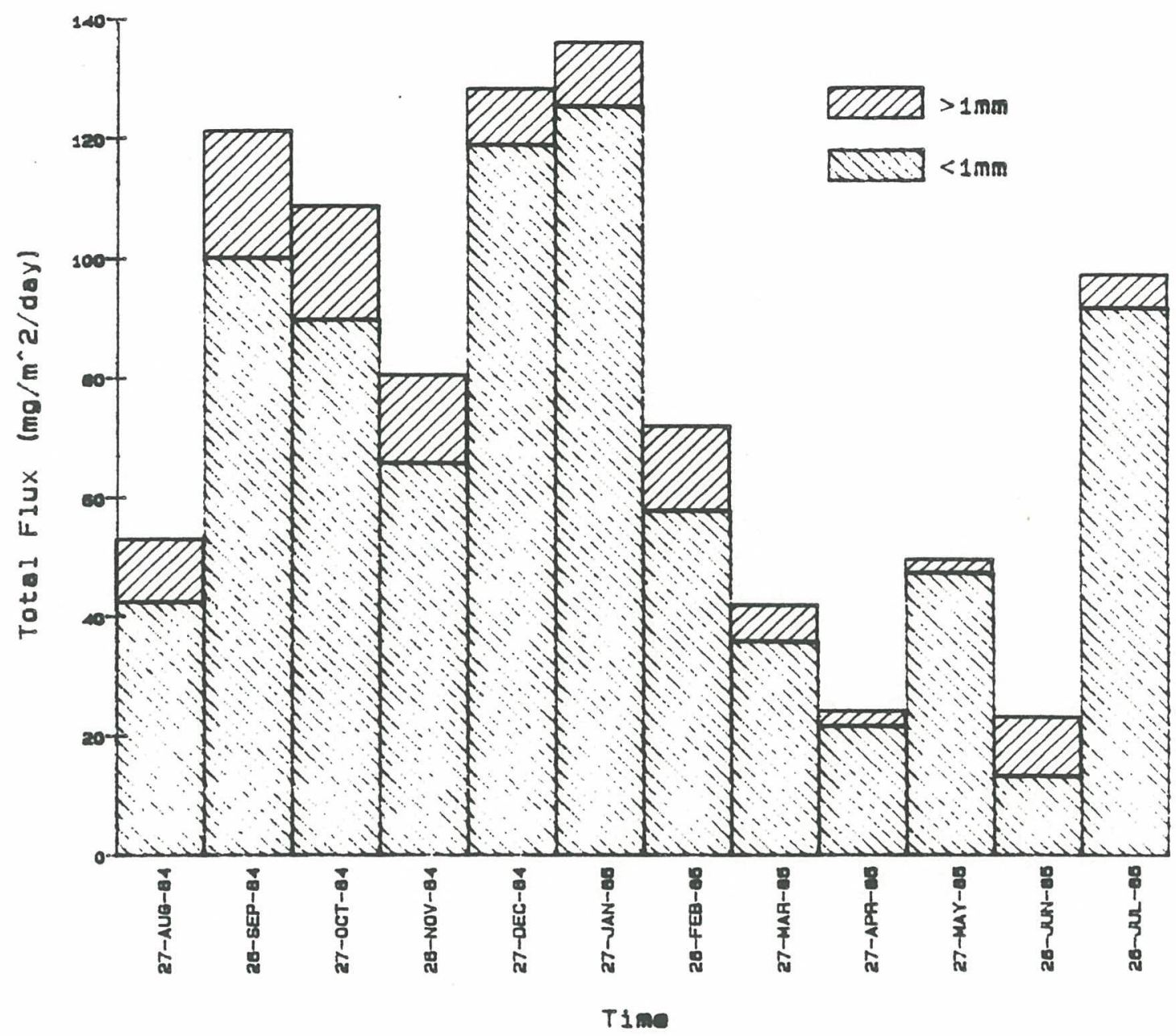

\begin{tabular}{|c|c|c|c|c|c|}
\hline $\begin{array}{l}\text { Sample } \\
\text { I.D. }\end{array}$ & $\begin{array}{l}\text { TTLF } \\
\leqslant 1\end{array}$ & $\begin{array}{l}1 \% \text { of } \\
\text { total }\end{array}$ & $\begin{array}{l}\text { TTLF } \\
>1\end{array}$ & $\begin{array}{l}1 \% \text { of } \\
\text { total }\end{array}$ & $\begin{array}{l}\text { TTLF } \\
\text { total }\end{array}$ \\
\hline 26 BI $1-1700-1$ & 42.41 & 79.97 & 10.62 & 20.03 & 53.03 \\
\hline 27 BI $1-1700-2$ & 100.12 & 82.55 & 21.17 & 17.45 & 121.29 \\
\hline 28 BI $1-1700-3$ & 89.79 & 82.63 & 18.88 & 17.37 & 108.67 \\
\hline 29 BI $1-1700-4$ & 65.74 & 81.50 & 14.82 & 18.40 & 80.56 \\
\hline 30 BI $1-1700-5$ & 118.82 & 92.68 & 9.38 & 7.32 & 128.20 \\
\hline 31 BI $1-1700-6$ & 125.34 & 92.12 & 10.72 & 7.88 & 136.06 \\
\hline 32 BI $1-1700-7$ & 57.81 & 80.41 & 14.08 & 19.59 & 71.89 \\
\hline 33 BI $1-1700-8$ & 35.88 & 85.41 & 6.13 & 14.59 & 42.01 \\
\hline 34 BI $1-1700-9$ & 21.65 & 89.65 & 2.50 & 10.35 & 24.15 \\
\hline 35 BI $1-1700-10$ & 47.31 & 95.42 & 2.27 & 4.58 & 49.58 \\
\hline 36 BI $1-1700-11$ & 13.22 & 56.98 & 9.98 & 43.02 & 23.20 \\
\hline 37 BI $1-1700-12$ & 91.78 & 94.35 & 5.50 & 5.65 & 97.28 \\
\hline
\end{tabular}

Flux is in $m g / m^{\wedge} 2 / d a y$. 
Carbonate FIUx at Bear Is?and (BI--1). 1700m, 1984-1985

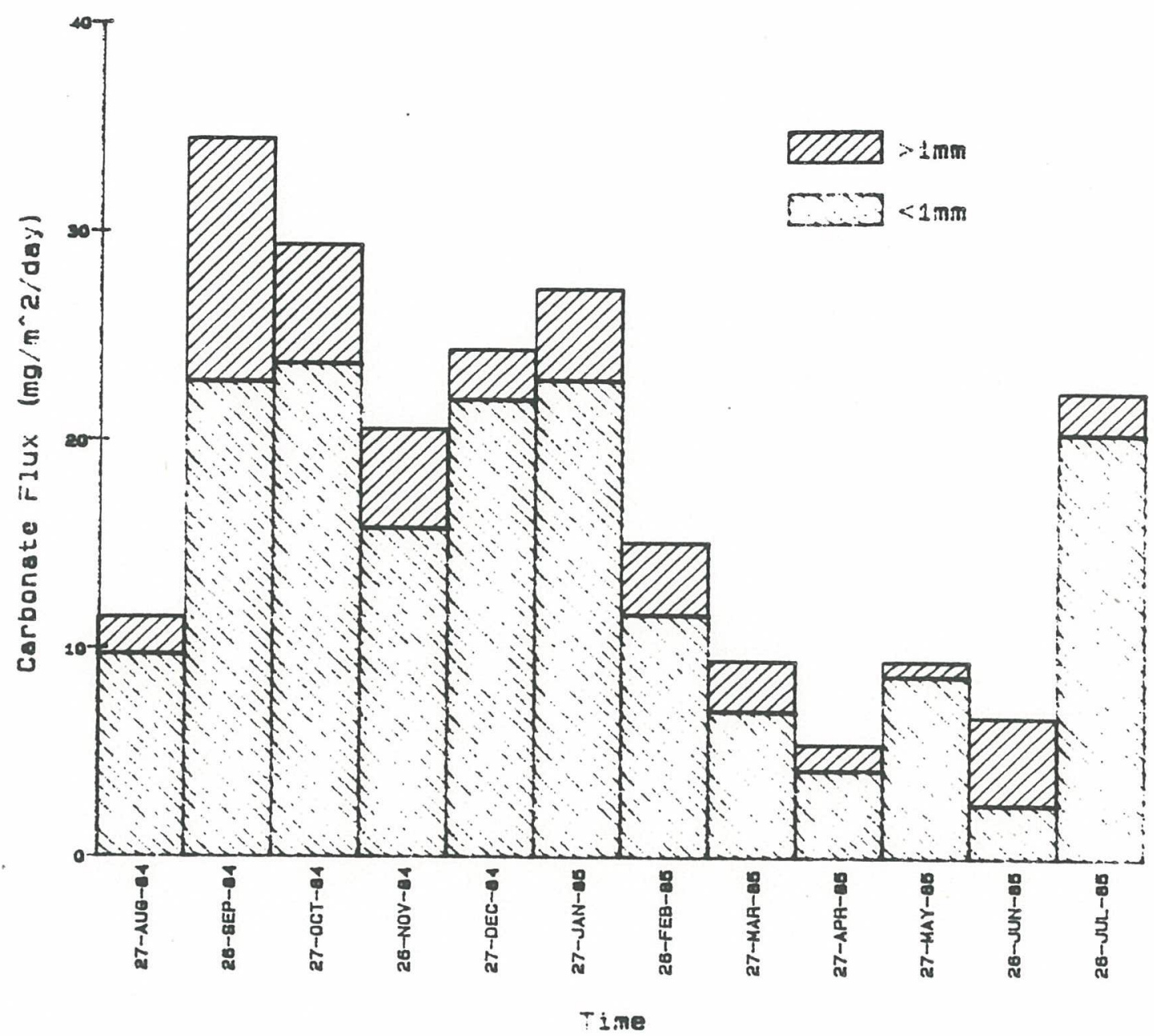

\begin{tabular}{|c|c|c|c|c|c|c|}
\hline $\begin{array}{l}\text { Sample } \\
\text { I.D. }\end{array}$ & $\begin{array}{l}\text { CRTA } \\
<1\end{array}$ & $\begin{array}{l}\text { CRTA \% } \\
\text { tot. }<1\end{array}$ & $\begin{array}{l}\text { CRTA } \\
>1\end{array}$ & $\begin{array}{l}\text { CRTA } \% \\
\text { tot. }>1\end{array}$ & $\begin{array}{l}\text { CRTA } \\
\text { total }\end{array}$ & $\begin{array}{l}\text { CRTA } \\
\text { total }\end{array}$ \\
\hline $268 I 1-1700-1$ & 9.80 & 18.48 & 1.77 & 3.34 & 11.56 & \\
\hline 27 BI 1-1700-2 & 22.83 & 18.82 & 11.61 & 9.57 & 34.44 & $<8$ \\
\hline 28 BI $1-1700-3$ & 23.67 & 21.78 & 5.66 & 5.21 & 29.55 & \\
\hline 29 BI 1-1700-4 & 15.80 & 19.61 & 4.75 & 5.90 & 20.73 & 2 \\
\hline 30 BI $1-1700-5$ & 21.95 & 17.12 & 2.42 & 1.89 & 24.52 & $\theta$. \\
\hline 31 BI 1-1700-6 & 22.88 & 16.82 & $4: 38$ & 3.22 & 27.31 & 20. \\
\hline $328 I 1-1700-7$ & 11.63 & 16.18 & 3.47 & 4.83 & 15.26 & 21. \\
\hline $33 B I 1-1700-8$ & 7.04 & 16.76 & 2.41 & 5.74 & 9.62 & 22. \\
\hline 34 BI $1-1700-9$ & 4.18 & 17.31 & 1.25 & 5.18 & 5.56 & 7 \\
\hline 35 BI $1-1700-10$ & 8.74 & 17.63 & 0.72 & 1.45 & 9.52 & \\
\hline 36 BI $1-1700-11$ & 2.56 & 11.03 & 4.20 & 18.10 & 6.81 & \\
\hline 37 BI $1-1700-12$ & 20.38 & 20.95 & 2.00 & 2.06 & 22.56 & \\
\hline
\end{tabular}

Flux is in $m g / m^{\wedge} 2 /$ day. 
Noncombustible Flux at Bear Island 1. 1700m, 1984-85

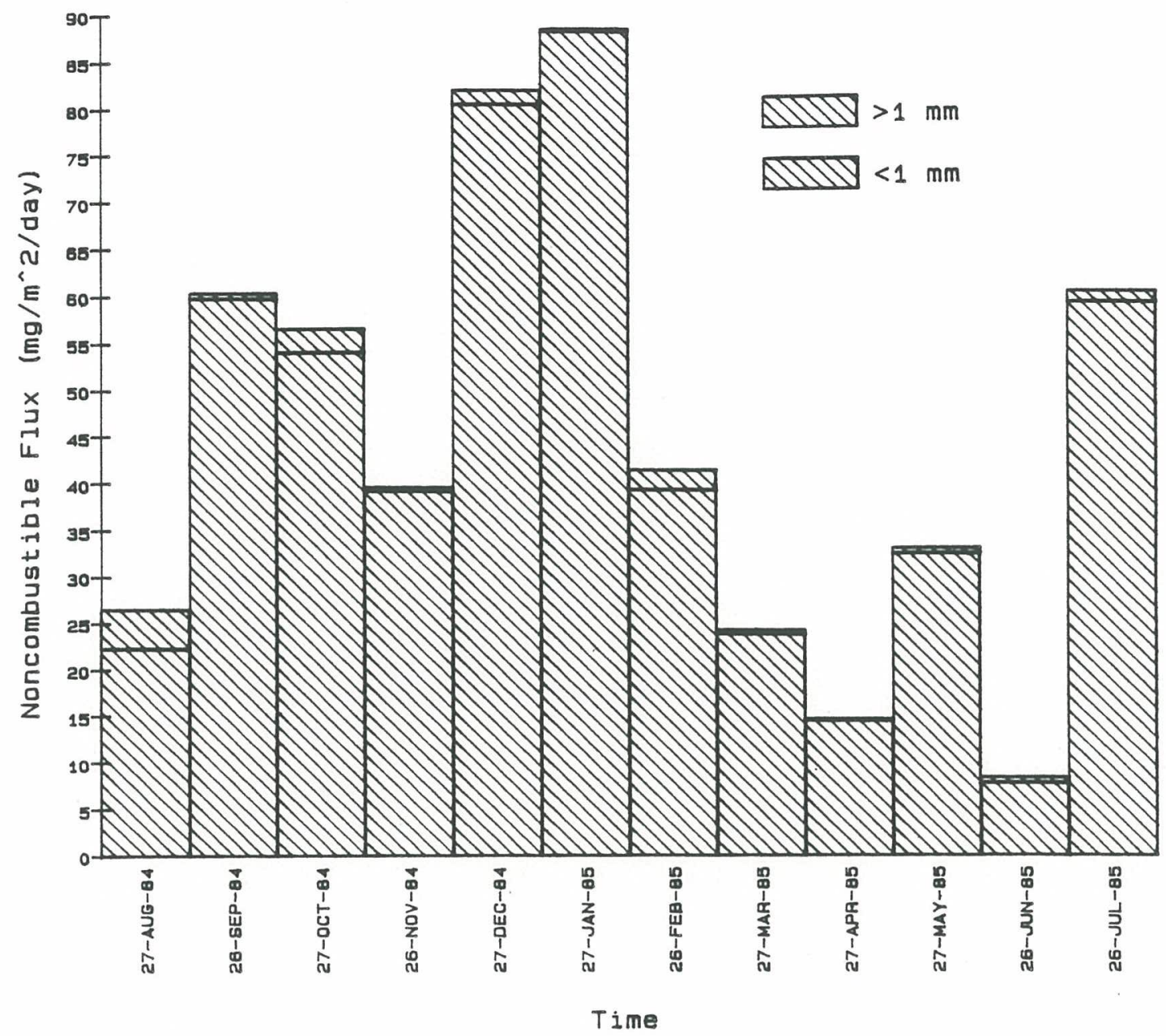

\begin{tabular}{|c|c|c|c|c|c|c|}
\hline $\begin{array}{l}\text { Sample } \\
\text { ID\# }\end{array}$ & $\begin{array}{l}\text { NONC } \\
<1\end{array}$ & $\begin{array}{l}\text { NONC } \% \\
\text { tot. } 1\end{array}$ & $\begin{array}{l}\text { NONC } \\
>1\end{array}$ & $\begin{array}{l}\text { NONC } \% \\
\text { tot. }>1\end{array}$ & $\begin{array}{l}\text { NONC } \\
\text { total }\end{array}$ & $\begin{array}{l}\text { NONC } \% \\
\text { total }\end{array}$ \\
\hline 26 BI $1-1700-1 *$ & 22.26 & 41.98 & 4.25 & 8.01 & 26.52 & 50.01 \\
\hline 27 BI $1-1700-2 *$ & 59.87 & 49.36 & 0.56 & 0.46 & 60.43 & 49.82 \\
\hline 28 BI $1-1700-3 *$ & 54.04 & 49.73 & 2.59 & 2.38 & 56.63 & 52.11 \\
\hline 29 BI $1-1700-4$ * & 39.17 & 48.62 & 0.39 & 0.48 & 39.56 & 49.11 \\
\hline $308 I 1-1700-5 *$ & 80.57 & 62.85 & 1.55 & 1.21 & 82.11 & 64.05 \\
\hline 31 EI $1-1700-6 *$ & 88.35 & 64.93 & 0.24 & 0.18 & 88.59 & 65.11 \\
\hline 32 BI $1-1700-7 *$ & 39.29 & 54.65 & 2.14 & 2.98 & 41.43 & 57.63 \\
\hline 33 BII-1700-8* & 23.82 & 56.70 & 0.46 & 1.09 & 24.28 & 57.80 \\
\hline 34 BI $1-1700-9 *$ & 14.51 & 60.08 & 0.13 & 0.54 & 14.63 & 60.58 \\
\hline 35 BII $-1700-10 *$ & 32.45 & 65.45 & 0.62 & 1.25 & 33.08 & 66.72 \\
\hline 36 BI $1-1700-11 *$ & 7.86 & 33.88 & 0.58 & 2.50 & 8.44 & 36.38 \\
\hline 37 BI $1-1700-12 *$ & 59.40 & 61.06 & 1.21 & 1.24 & 60.61 & 62.30 \\
\hline
\end{tabular}

Flux is in $m g / m^{\wedge} 2 /$ day. 
Combustible Flux at Bear Island (BI-1). 1700m, 1984-85

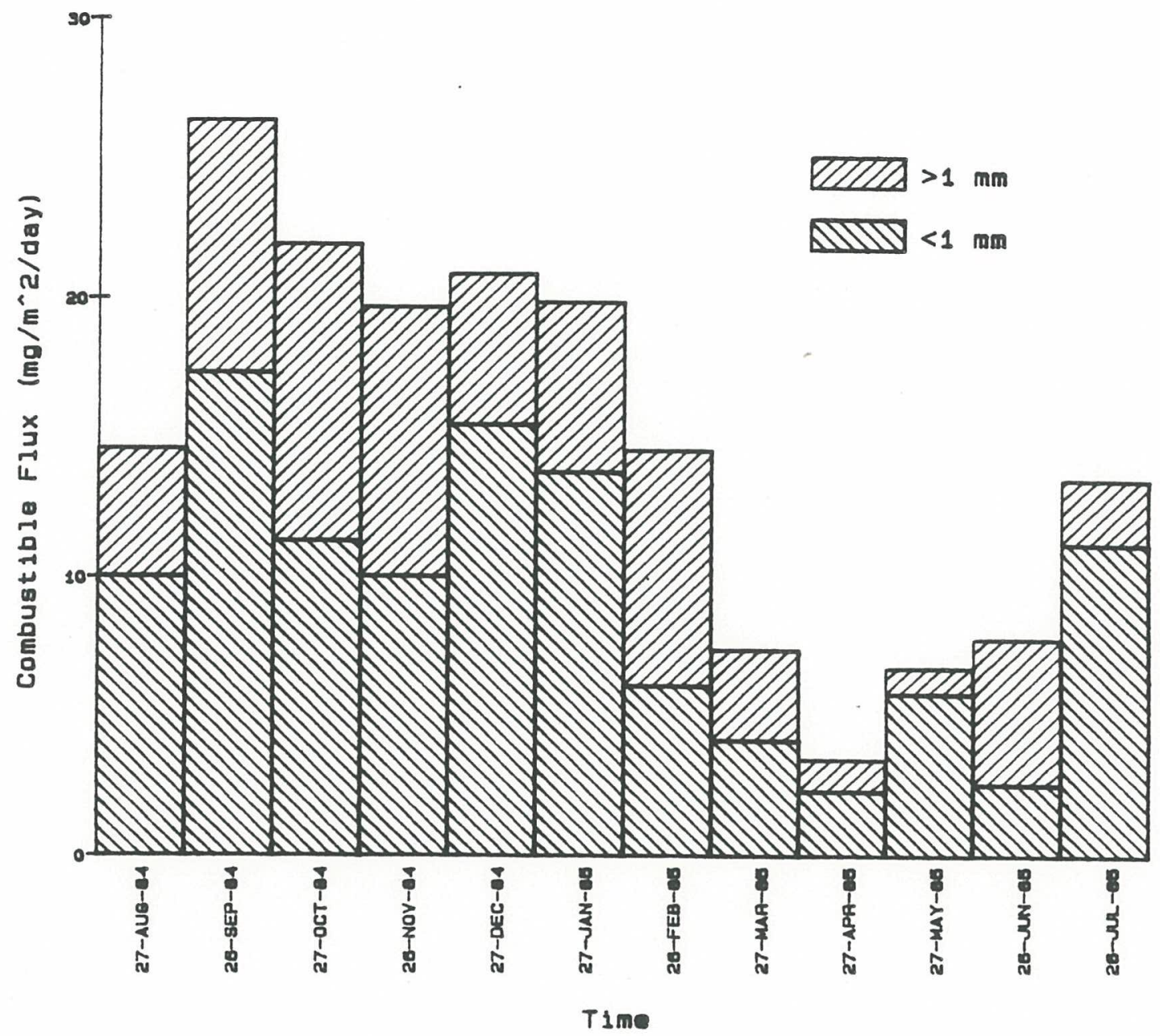

\begin{tabular}{|c|c|c|c|c|c|c|}
\hline $\begin{array}{l}\text { Sample } \\
\text { ID\# }\end{array}$ & $\begin{array}{l}\text { COMB } \\
<1\end{array}$ & $\begin{array}{l}\text { COMB } \% \\
\text { tot. }<1\end{array}$ & $\begin{array}{l}\text { COMB } \\
>1\end{array}$ & $\begin{array}{l}\text { COMB } \% \\
\text { tot. } 1\end{array}$ & $\begin{array}{l}\text { COMB } \\
\text { TOTAL }\end{array}$ & $\begin{array}{l}\text { CoMB } \% \\
\text { total }\end{array}$ \\
\hline 26 BI $1-1700-1 *$ & 10.03 & 18.91 & 4.60 & 8.67 & 14.64 & $27 . E 1$ \\
\hline 27 EI $1-1700-2 *$ & 17.33 & 14.29 & 9.00 & 7.42 & 26.33 & 21.0 \\
\hline 28 BI $1-1700-3 *$ & 11.29 & 10.39 & 10.62 & 9.77 & 21.91 & 20.16 \\
\hline 29 BI $1-1700-4$ * & 10.03 & 12.45 & 9.67 & 12.00 & 19.71 & 24.47 \\
\hline 30 BI $1-1700-5 *$ & 15.48 & 12.07 & 5.42 & 4.23 & 20.90 & 16.30 \\
\hline 31 BI $1-1700-6 *$ & 13.78 & 10.13 & 6.10 & 4.48 & 19.87 & 14.50 \\
\hline 32 BI $1-1700-7 *$ & 6.08 & 8.46 & 8.47 & 11.78 & 14.55 & 20.24 \\
\hline $3 J$ EI $1-1700-8 *$ & 4.14 & 9.35 & 3.27 & 7.78 & 7.41 & 17.64 \\
\hline 34 BI $1-1700-9 *$ & 2.31 & 9.57 & 1.12 & 4.54 & 3.43 & 14.20 \\
\hline 35 BI $1-1700-10 *$ & 5.80 & 11.70 & 0.93 & 1.88 & 6.73 & 13.57 \\
\hline 36 BI $1-1700-11 *$ & 2.58 & 11.12 & 5.20 & 22.41 & 7.78 & 33.53 \\
\hline 37 BI $1-1700-12 *$ & 11.22 & 11.53 & 2.29 & 2.35 & 13.51 & 13.89 \\
\hline
\end{tabular}

Flux is in $m g / m^{\wedge} 2 / d a y$. 
Biogenic Silica Flux at Bear Island 1. 1700m. 1984-85

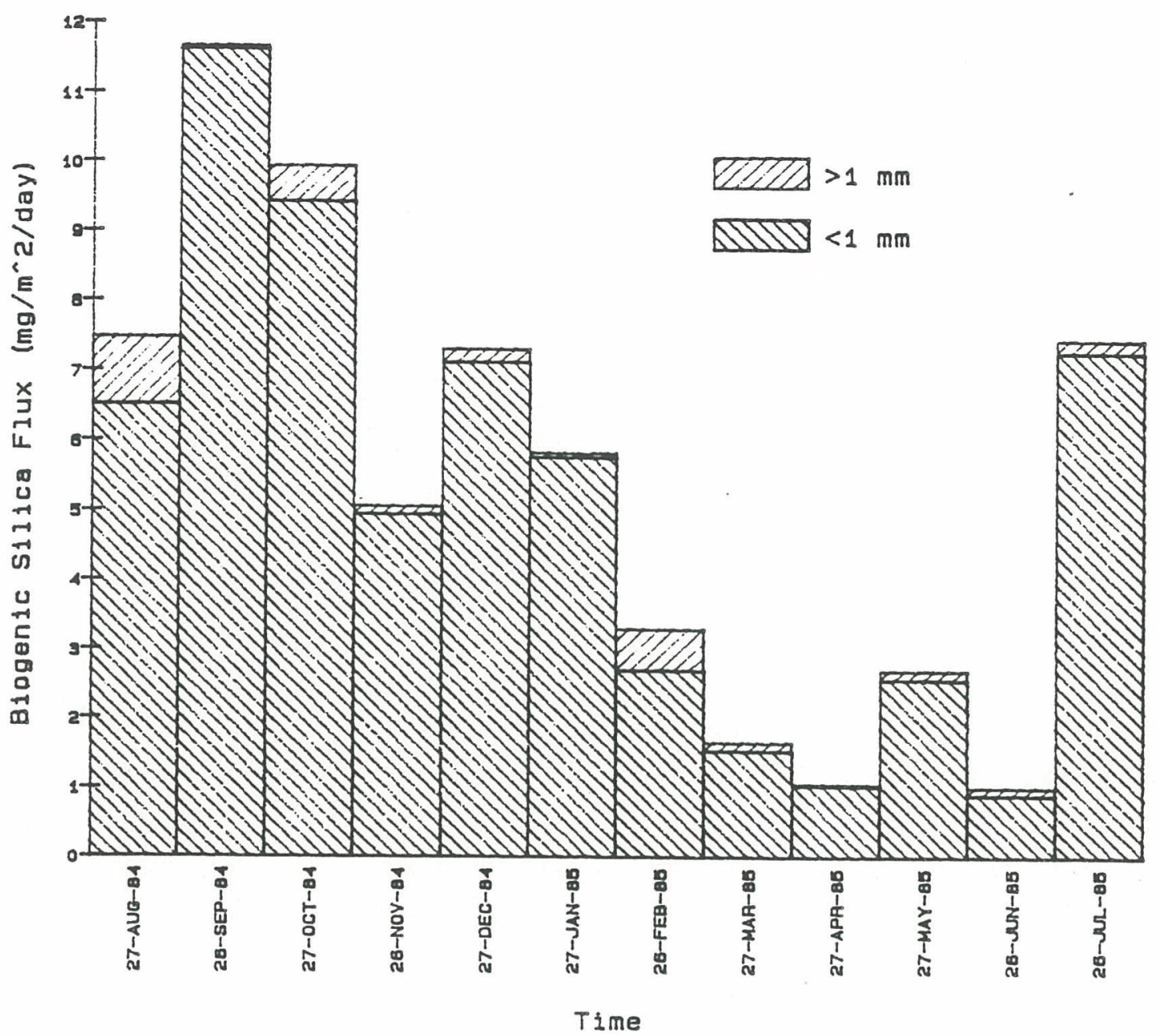

\begin{tabular}{|c|c|c|c|c|c|c|c|}
\hline $\begin{array}{l}\text { Sample } \\
\text { ID\# }\end{array}$ & $\begin{array}{l}\text { OPAL } \\
\vdots 1\end{array}$ & $\begin{array}{l}\text { OPAL<1 } \\
\text { \% NCMb. }\end{array}$ & $\begin{array}{l}\text { OPAL } \\
>1\end{array}$ & $\begin{array}{l}\text { OPAL>1 } \\
\% \text { NCMB. }\end{array}$ & $\begin{array}{l}\text { OPAL } \\
\text { total }\end{array}$ & $\begin{array}{l}\text { OPAL } \\
\% \text { NCMb. }\end{array}$ & $\begin{array}{l}\text { OPAL } \\
\% \text { total }\end{array}$ \\
\hline 26 BI $1-1700-1$ & 6.51 & 24.56 & 0.959340 & 3.62 & 7.47 & 28.17 & 14.09 \\
\hline 27 BII-1700-2 & 11.62 & 19.22 & 0.051184 & 0.10 & 11.68 & 19.32 & 9.63 \\
\hline 28 BII-1700-3 & 9.41 & 16.62 & 0.520868 & 0.92 & 9.93 & 17.54 & 9.14 \\
\hline 29 BI1-1700-4 & 4.94 & 12.49 & 0.123738 & 0.31 & 5.07 & 12.81 & 6.29 \\
\hline 30 BI $1-1700-5$ & 7.12 & 8.67 & 0.191400 & 0.23 & 7.31 & 8.90 & 5.70 \\
\hline 31 BI 1-1700-6 & 5.75 & 6.50 & 0.065302 & 0.07 & 5.82 & 6.57 & 4.28 \\
\hline 32 BI 1-1700-7 & 2.69 & 6.50 & 0.595221 & 1.44 & 3.29 & 7.93 & 4.57 \\
\hline 33 BI $1-1700-8$ & 1.53 & 6.28 & 0.119040 & 0.49 & 1.64 & 6.77 & 3.91 \\
\hline 34 BI1-1700-9 & 1.04 & 7.08 & 0.020000 & 0.14 & 1.06 & 7.21 & 4.37 \\
\hline 35 BI $1-1700-10$ & 2.56 & 7.74 & 0.136090 & 0.41 & 2.70 & 8.15 & 5.44 \\
\hline 36 BI $1-1700-11$ & 0.90 & 10.66 & 0.115022 & 1.36 & 1.01 & 12.02 & 4.37 \\
\hline 37 BI $1-1700-12$ & 7.27 & 12.00 & 0.186550 & 0.31 & 7.46 & 12.31 & 7.67 \\
\hline
\end{tabular}

Flux is in $m g / m^{\wedge} 2 /$ day.

\%Namb. is "\% noncombustible flux". 
Lithogenic FIux at Bear Island 1, 1700m, 1984-85

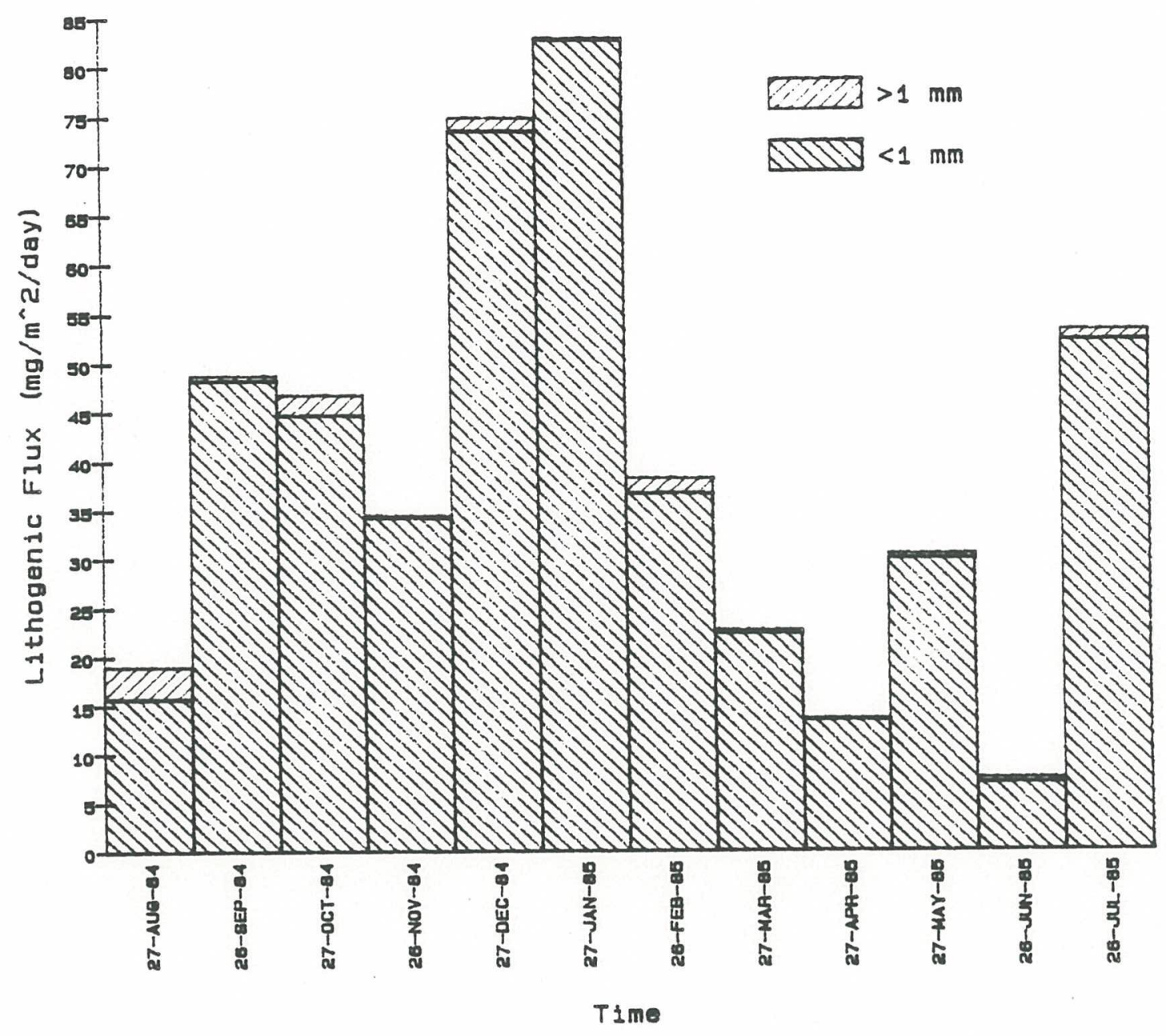

\begin{tabular}{|c|c|c|c|c|c|c|}
\hline $\begin{array}{l}\text { Sample } \\
\text { ID\# }\end{array}$ & $\begin{array}{l}\text { LITH } \\
<1\end{array}$ & $\begin{array}{l}\text { LITHCl } \\
\% \text { NCMb. }\end{array}$ & $\begin{array}{l}\text { LITH } \\
>1\end{array}$ & $\begin{array}{l}\text { LITH>l } \\
\% \text { NCMb. }\end{array}$ & $\begin{array}{l}\text { LITH } \\
\text { total }\end{array}$ & $\begin{array}{l}\text { LITH } \\
\% \text { Ncmb. }\end{array}$ \\
\hline BI 1-1700-1 & 15.75 & 59.38 & 3.29 & 12.41 & 19.04 & 71.79 \\
\hline $7 B I 1-1700-2$ & 48.25 & 79.85 & 0.50 & 0.83 & 48.75 & 80.68 \\
\hline 8 BI $1-1700-3$ & 44.53 & 78.81 & 2.07 & 3.65 & 46.70 & 82.46 \\
\hline $9 B I 1-1700-4$ & 34.23 & 86.52 & 0.27 & 0.67 & 34.49 & 87.19 \\
\hline $30 B I 1-1700-5$ & 73.45 & 89.45 & 1.36 & 1.55 & 74.81 & 91.11 \\
\hline EI1-1700-6 & 82.60 & 93.23 & 0.17 & 0.20 & 82.77 & 93.43 \\
\hline $2 B I 1-1700-7$ & 36.60 & 88.34 & 1.54 & 3.73 & 38.14 & 92.07 \\
\hline 3 BI1-1700-8 & 22.29 & 91.82 & 0.34 & 1.40 & 22.64 & 93.23 \\
\hline $48 I 1-1700-9$ & 13.47 & 92.10 & 0.11 & 0.75 & 13.58 & 92.86 \\
\hline$=8 I 1-1700-10$ & 29.89 & 90.35 & 0.48 & 1.46 & 30.37 & 91.82 \\
\hline$B I 1-1700-11$ & 6.96 & 82.47 & 0.46 & 5.51 & 7.43 & 87.98 \\
\hline BI $1-1700-12$ & 52.13 & 86.01 & 1.02 & 1.69 & 53.15 & 87.59 \\
\hline
\end{tabular}

Flux is in $m g / m " 2 / d a y$.

$\%$ Ncmb. is "\% of noncombustible flux". 
Carbon Flux at Bear Island 1. 1700m. 1984-85

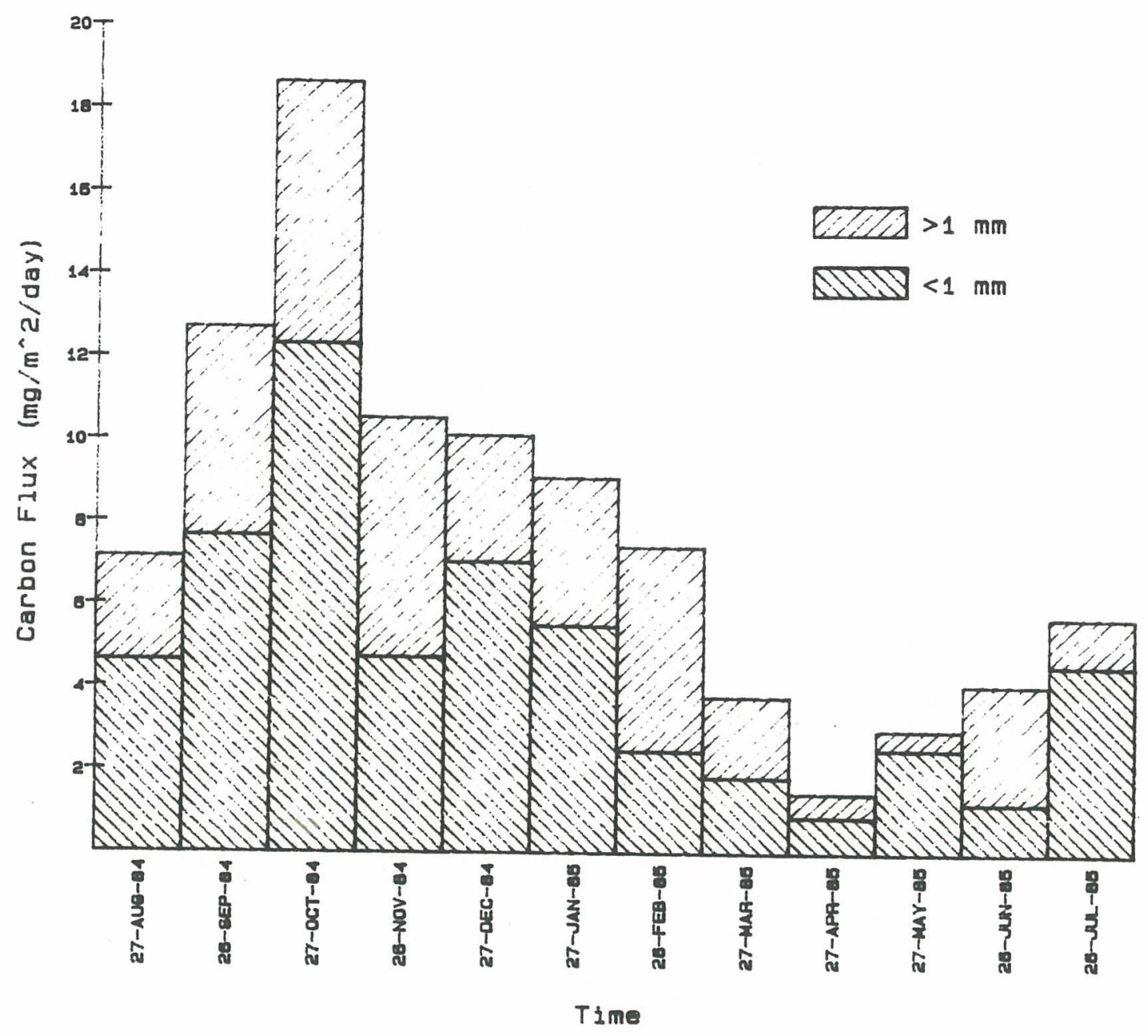

\begin{tabular}{|c|c|c|c|c|c|c|}
\hline $\begin{array}{l}\text { Sample } \\
\text { I.D. }\end{array}$ & $\begin{array}{l}\text { CRNC } \\
<1\end{array}$ & $\begin{array}{l}\text { CRNC }<1 \\
\% \text { cmbf. }\end{array}$ & $\begin{array}{l}\text { CRNC } \\
y 1\end{array}$ & $\begin{array}{l}\text { CRNC> } 1 \\
\% c m b f .\end{array}$ & $\begin{array}{l}\text { CRNC } \\
\text { total }\end{array}$ & $\begin{array}{l}\text { CRNCtot. } \\
\% \text { cmb } f .\end{array}$ \\
\hline 26 BI $1-1700-1 *$ & 4.65 & 31.76 & 2.54 & 17.35 & 7.19 & 49. \\
\hline 27 BI $1-1700-2 *$ & 7.66 & 29.09 & 5.06 & 19.20 & 12.72 & 43.2 \\
\hline 28 BI $1-1700-3 *$ & 12.31 & 56.18 & 6.35 & 28.98 & 18.65 & 85.17 \\
\hline 29 BI $1-1700-4 *$ & 4.72 & 23.96 & 5.82 & 29.55 & 10.55 & 53.51 \\
\hline 30 BI $1-1700-5 *$ & 7.04 & 33.68 & 3.07 & 14.69 & 10.11 & 48.3 \\
\hline 31 BI $1-1700-6 *$ & 5.50 & 27.67 & 3.61 & 18.15 & 9.11 & 45.8 \\
\hline 32 BI $1-1700-7 *$ & 2.48 & 17.04 & 4.97 & 34.16 & 7.45 & 51.2 \\
\hline 33 BI $1-1700-8 *$ & 1.83 & 24.68 & 1.96 & 26.46 & 3.79 & 51.1 \\
\hline $348 I 1-1700-9 *$ & 0.88 & 25.66 & 0.59 & 17.20 & 1.47 & 42.6 \\
\hline 35 BI $1-1700-10 *$ & 2.54 & 37.71 & 0.47 & 7.02 & 3.01 & 44.7 \\
\hline 36 BI $1-1700-11 *$ & 1.23 & 15.81 & 2.88 & 37.02 & 4.11 & 52.8 \\
\hline 37 BI $1-1700-12 *$ & 4.57 & 33.84 & 1.16 & 8.61 & 5.74 & 42.4 \\
\hline
\end{tabular}

Flux is in mg/m"2/day.

"\%ombf" = "\% of combustible flux" 


\section{Nitrogen Flux at Bear Island 1. 1700m, 1984-1985}

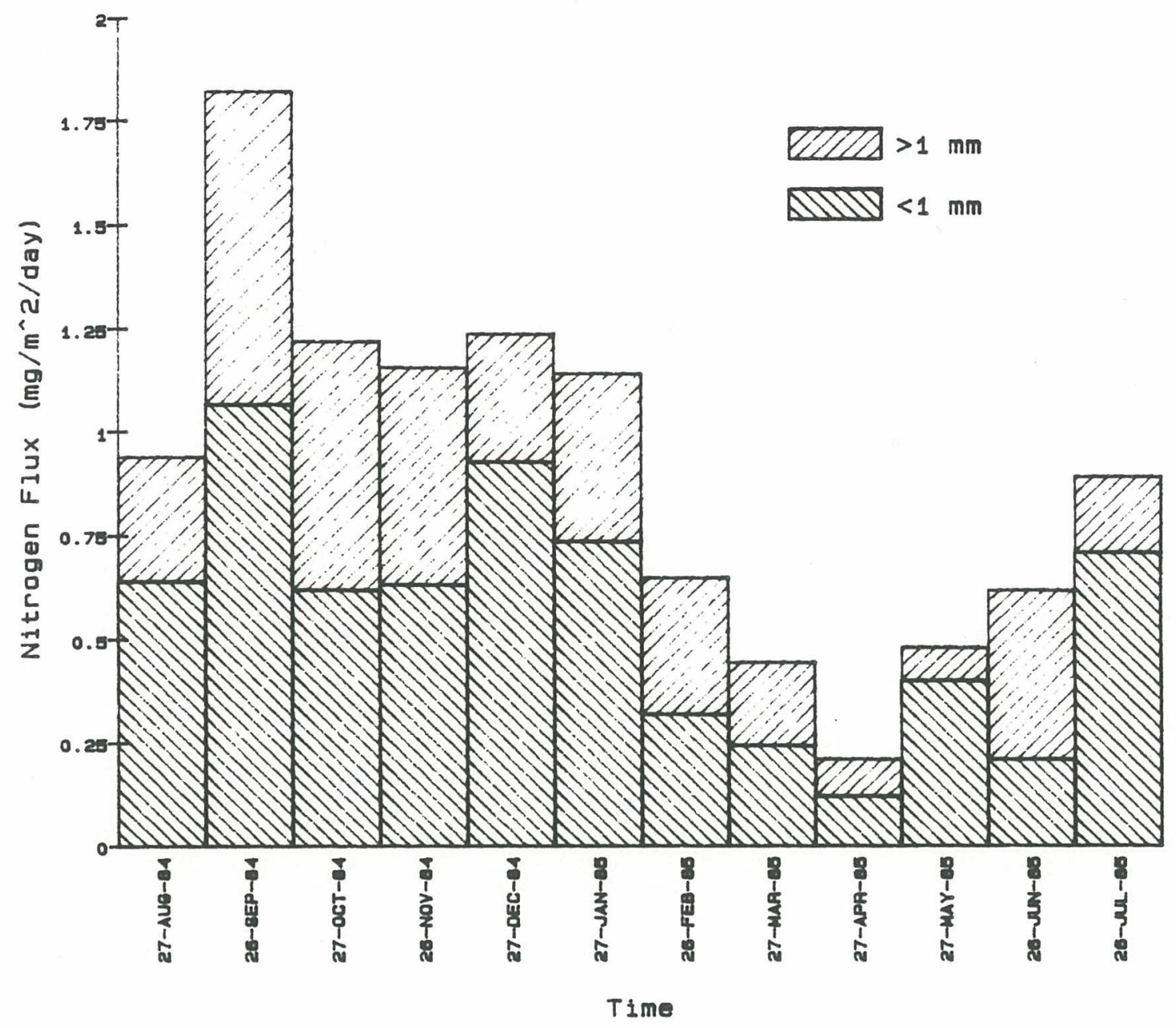

\begin{tabular}{|c|c|c|c|c|c|c|}
\hline $\begin{array}{l}\text { Sample } \\
\text { I.D. }\end{array}$ & $\begin{array}{l}\text { NTGN } \\
\ll 1\end{array}$ & $\begin{array}{l}\text { NTGN<1 } \\
\% \text { cmb f. }\end{array}$ & $\begin{array}{l}\text { NTGN } \\
>1\end{array}$ & $\begin{array}{l}\text { NTGND1 } \\
\% \text { CMbf. }\end{array}$ & $\begin{array}{l}\text { NTGN } \\
\text { total }\end{array}$ & $\begin{array}{l}\text { NTGNtat. } \\
\% \text { omb f. }\end{array}$ \\
\hline 26 BI1-1700-1* & 0.54 & 4.37 & 0.30 & 2.05 & 0.94 & 6.42 \\
\hline $27 B I 1-1700-2 *$ & 1.07 & 4.05 & 0.76 & 2.87 & 1.82 & 6.92 \\
\hline 28 BI $1-1700-3 *$ & 0.52 & 2.83 & 0.60 & 2.74 & 1.22 & 5.57 \\
\hline 29 BI $1-1700-4 *$ & 0.63 & 3.21 & 0.53 & 2.66 & 1.16 & 5.87 \\
\hline 30 BI $1-1700-5 *$ & 0.93 & 4.45 & 0.31 & 1.48 & 1.24 & 5.93 \\
\hline 31 BI $1-1700-5 *$ & 0.74 & 3.71 & 0.41 & 2.04 & 1.14 & 5.75 \\
\hline 32 BI $1-1700-7 *$ & 0.32 & 2.20 & 0.33 & 2.27 & 0.65 & 4.47 \\
\hline 33 BI $1-1700-8 *$ & 0.24 & 3.29 & 0.20 & 2.73 & 0.45 & 6.01 \\
\hline 34 EI $1-1700-9 *$ & 0.12 & 3.50 & 0.09 & 2.62 & 0.21 & 6.12 \\
\hline 35 BI $1-1700-10 *$ & 0.40 & 5.95 & 0.08 & 1.21 & 0.48 & 7.15 \\
\hline $3 E$ BI $1-1700-11 *$ & 0.21 & 2.70 & 0.41 & 5.27 & 0.62 & 7.97 \\
\hline 37 BI $1-1700-12 *$ & 0.71 & 5.26 & 0.18 & 1.35 & 0.89 & $E .59$ \\
\hline
\end{tabular}

Flux is in $m g / m^{*} 2 / d a y$.

"\%mbf" $=" \%$ of combustible flux". 
Hydrogen Flux at Bear Island 1. 1700m, 1984-85

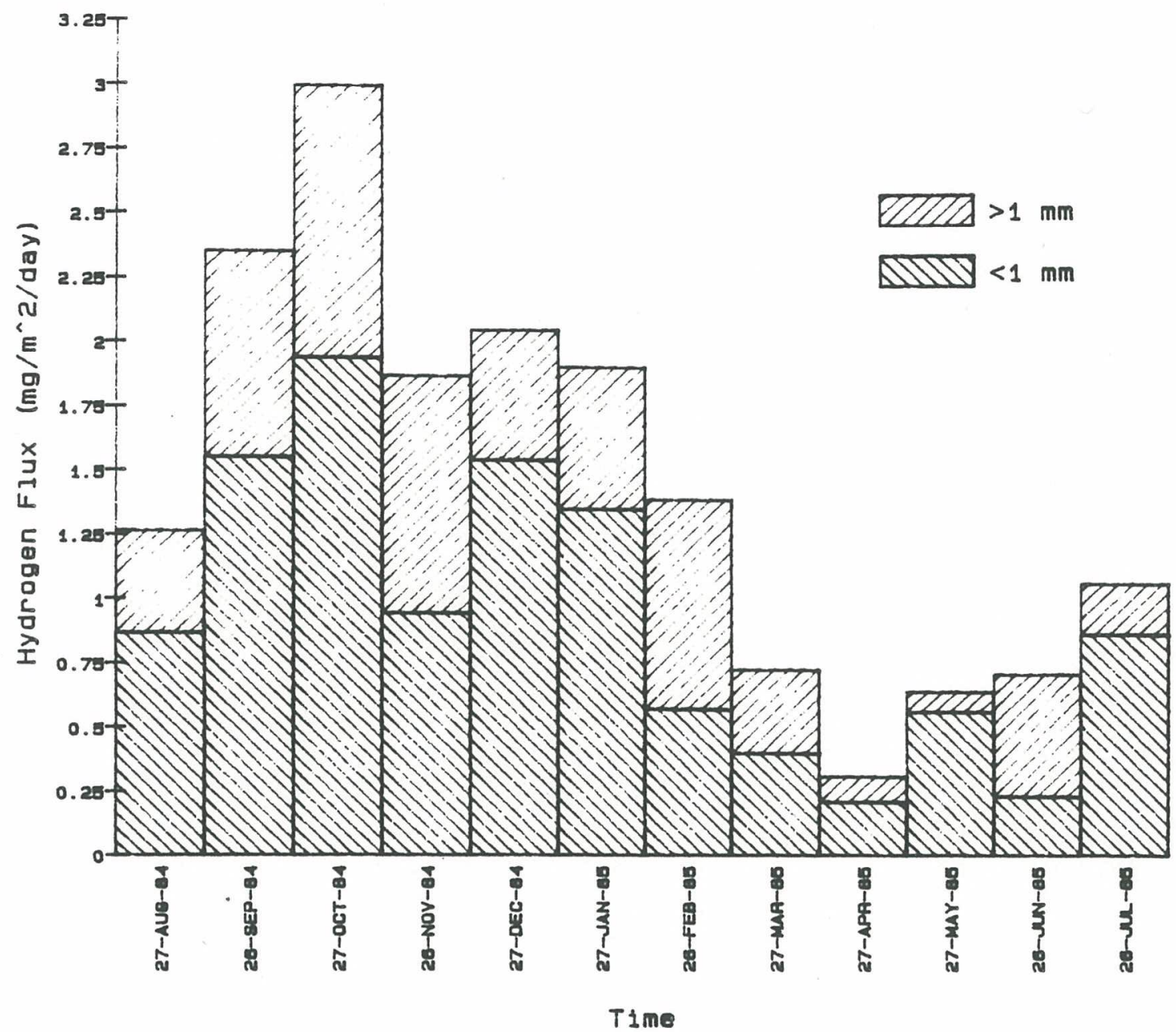

\begin{tabular}{|c|c|c|c|c|c|c|}
\hline $\begin{array}{l}\text { Sample } \\
\text { I.O. }\end{array}$ & $\begin{array}{l}\text { HYDC } \\
<1\end{array}$ & $\begin{array}{l}\text { HYDC } 1 \\
\% c m b f .\end{array}$ & $\begin{array}{l}\text { HYDC } \\
>1\end{array}$ & $\begin{array}{l}\text { HYDC } 1 \\
\% \text { cmbf. }\end{array}$ & $\begin{array}{l}\text { HYDC } \\
\text { total }\end{array}$ & $\begin{array}{l}\text { HYDCtot. } \\
\% \text { cmbf. }\end{array}$ \\
\hline 26 BI $1-1700-1 *$ & 0.87 & 5.94 & 0.40 & 2.73 & 1.27 & 8.67 \\
\hline 27 BI $1-1700-2 *$ & 1.55 & 5.90 & 0.80 & 3.04 & 2.35 & 8.94 \\
\hline 28 BI $1-1700-3 *$ & 1.94 & 8.85 & 1.06 & 4.84 & 3.00 & 13.69 \\
\hline 29 BI $1-1700-4 *$ & 0.95 & 4.80 & 0.93 & 4.71 & 1.87 & 9.50 \\
\hline 30 BI $1-1700-5 *$ & 1.54 & 7.37 & 0.51 & 2.44 & 2.05 & 9.81 \\
\hline $318 I 1-1700-5 *$ & 1.35 & 6.80 & 0.56 & 2.80 & 1.91 & 9.60 \\
\hline 32 BI $1-1700-7 *$ & 0.57 & 3.92 & 0.82 & 5.64 & 1.39 & 9.55 \\
\hline 33 BI $1-1700-8 *$ & 0.40 & 5.38 & 0.33 & 4.43 & 0.73 & 9.81 \\
\hline 34 BI $1-1700-9 *$ & 0.21 & 6.12 & 0.10 & 2.92 & 0.31 & 9.04 \\
\hline 35 BI $1-1700-10 *$ & 0.56 & 8.35 & 0.08 & 1.20 & 0.64 & 9.55 \\
\hline 36 BI $1-1700-11 *$ & 0.23 & 2.96 & 0.48 & 6.17 & 0.71 & 9.13 \\
\hline 37 BI $1-1700-12 *$ & 0.86 & 6.38 & 0.20 & 1.47 & 1.05 & 7.85 \\
\hline
\end{tabular}

Flux is in $m g / m^{*} 2 /$ day.

"\%cmbf" = "\% of combustible flux" 


\section{NA-1 \\ AEGIR RIDGE \\ $65^{\circ} 31^{\prime} \mathrm{N}, \quad 00^{\circ} 64^{\prime} \mathrm{E}$}

Trap depth: 2,630m Water depth: 3,058m

Annual Fluxes $\left(\mathrm{g} / \mathrm{m}^{2} / \mathrm{yr}\right)$ :

Total................17.36

Carbonate..............9.18

Noncombustible $\ldots \ldots . . .5 .94$

Combustible ...........2.31

Lithogenic ............4.26

Biogenic Opalı......1.68

Organic C.............0.59

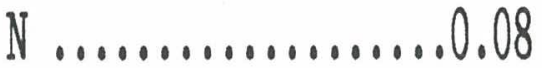

PARFLUX Mark 6-13

\begin{tabular}{|c|c|c|c|c|}
\hline $\begin{array}{l}\text { Sample } \\
\text { ID }\end{array}$ & $\begin{array}{l}\text { Opening } \\
\text { Date }\end{array}$ & $\begin{array}{l}\text { Closing } \\
\text { Date }\end{array}$ & Span & $\begin{array}{l}\text { Mid. } \\
\text { Date }\end{array}$ \\
\hline $57 N A 1-3058-1$ & $21-A \cup G-85$ & $13-S E P-85$ & 23 & $02-5 E P-85$ \\
\hline $58 N A 1-3058-2$ & $13-S E P-85$ & $\emptyset 6-0 C T-85$ & 23 & $25-5 E P-85$ \\
\hline $59 N A 1-3058-3$ & $O E-O C T-85$ & $29-0 C T-85$ & 23 & $18-0 C T-85$ \\
\hline $50 N A 1-3058-4$ & $29-0 C T-85$ & 21-NOU-85 & 23 & $10-$ NOU -85 \\
\hline 61 NA1-3058-5 & $21-\mathrm{NOU}-85$ & $14-0 E C-85$ & 23 & $03-0 E C-85$ \\
\hline 62 NA1-3058-6 & $14-D E C-85$ & $\triangle G-J A N-86$ & 23 & $26-D E C-85$ \\
\hline $63 N A 1-3058-7$ & $O G-J A N-86$ & $29-J A N-86$ & 23 & $18-J A N-86$ \\
\hline E4 NA1-3058-8 & $29-J A N-86$ & $21-F E B-86$ & 23 & $10-F E B-86$ \\
\hline $65 N A 1-3058-9$ & $21-F E B-86$ & $16-M A R-86$ & 23 & $05-M A R-86$ \\
\hline 5E NA1-3058-10 & $16-M A R-86$ & $08-A P R-86$ & 23 & $28-M A R-86$ \\
\hline $67 N A 1-3058-11$ & $08-A P R-86$ & $01-M A Y-86$ & 23 & $20-A P R-8 G$ \\
\hline $68 N A 1-3058-12$ & $01-M A Y-86$ & $24-M A Y-86$ & 23 & $13-M A Y-85$ \\
\hline 69 $N A 1-3058-13$ & 24-MAY-86 & $16-J \cup N-86$ & 23 & $05-J U N-86$ \\
\hline
\end{tabular}


Total Flux at Aegir Ridge (NA1). 3058m. 1985-1986

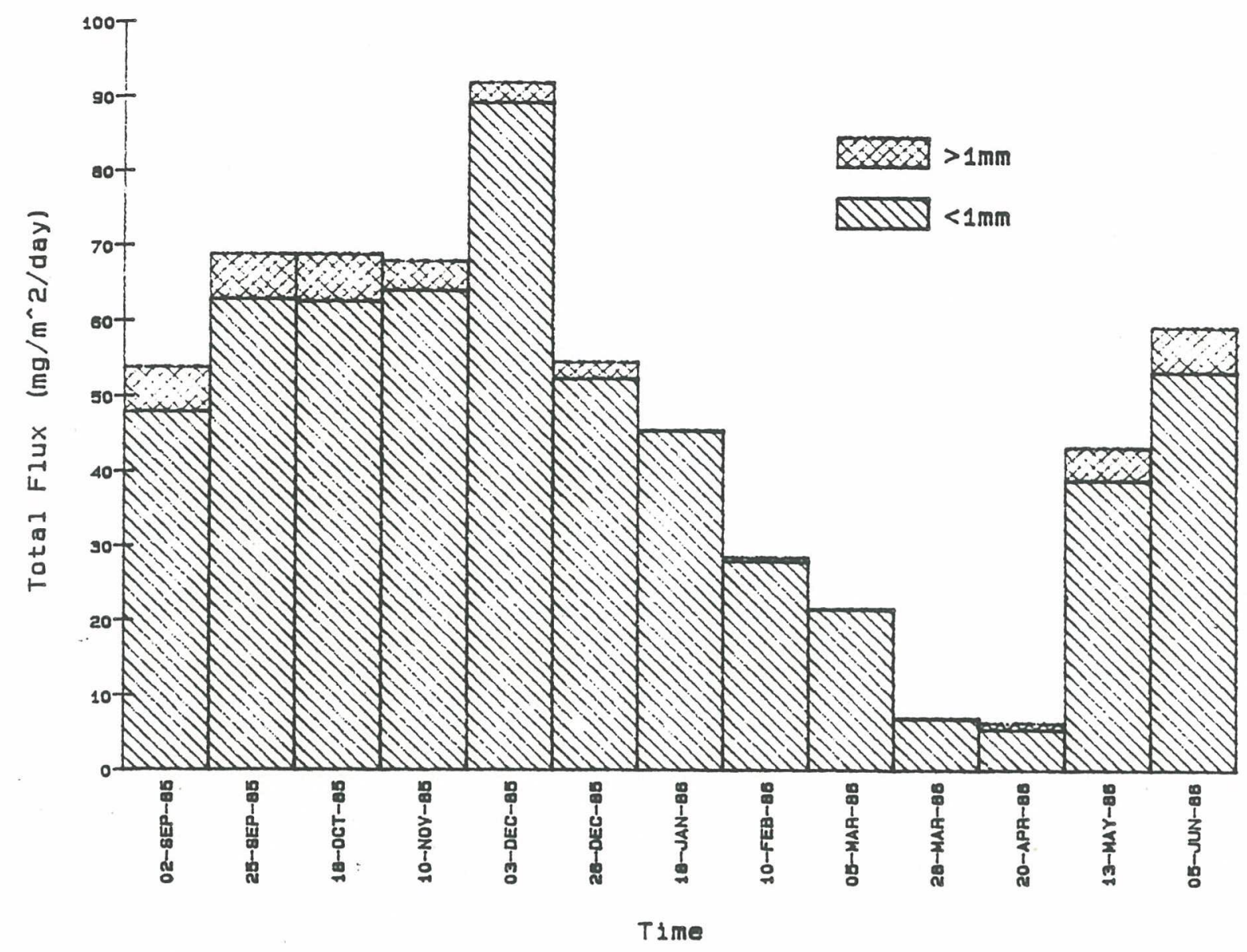

\begin{tabular}{|c|c|c|c|c|c|}
\hline $\begin{array}{l}\text { Sample } \\
\text { I.D. }\end{array}$ & $\begin{array}{l}\text { TTLF } \\
<1\end{array}$ & $\begin{array}{l}<1 \% \text { of } \\
\text { total }\end{array}$ & $\begin{array}{l}\text { TTLF } \\
>1\end{array}$ & $\begin{array}{l}>1 \% \text { of } \\
\text { total }\end{array}$ & $\begin{array}{l}\text { TTLF } \\
\text { total }\end{array}$ \\
\hline $57 \mathrm{NA1}-3058-1$ & 47.97 & 89.03 & 5.91 & 10.97 & 53.89 \\
\hline $58 N A 1-3058-2$ & 62.76 & 91.27 & 6.01 & 8.73 & 68.76 \\
\hline $59 N A 1-3058-3$ & 62.53 & 90.83 & 6.32 & 9.17 & 68.84 \\
\hline $60 N A 1-3058-4$ & 63.93 & 94.15 & 3.97 & 5.85 & 67.90 \\
\hline $61 N A 1-3058-5$ & 89.06 & 97.02 & 2.73 & 2.98 & 91.79 \\
\hline $62 N A 1-3058-6$ & 52.31 & 95.90 & 2.23 & 4.10 & 54.54 \\
\hline $63 N A 1-3058-7$ & 45.37 & 99.78 & 0.10 & 0.22 & 45.47 \\
\hline $64 N A 1-3058-8$ & 27.87 & 97.70 & 0.65 & 2.30 & 28.52 \\
\hline $65 N A 1-3058-9$ & 21.54 & 99.56 & 0.10 & 0.44 & 21.63 \\
\hline $66 N A 1-3058-10$ & 6.89 & 98.73 & 0.09 & 1.27 & 6.98 \\
\hline $67 N A 1-3058-11$ & 5.44 & 85.35 & 0.93 & 14.65 & 6.38 \\
\hline $68 N A 1-3058-12$ & 38.72 & 89.64 & 4.48 & 10.36 & 43.20 \\
\hline $69 N A 1-3058-13$ & 53.18 & 89.73 & 6.09 & 10.27 & 59.27 \\
\hline
\end{tabular}

Flux is in $\mathrm{mg} / \mathrm{m}^{\prime \prime 2} /$ day. 
Carbonate Flux at Aegir fidge 1 (NA-1), 3058 m. 1985-1986

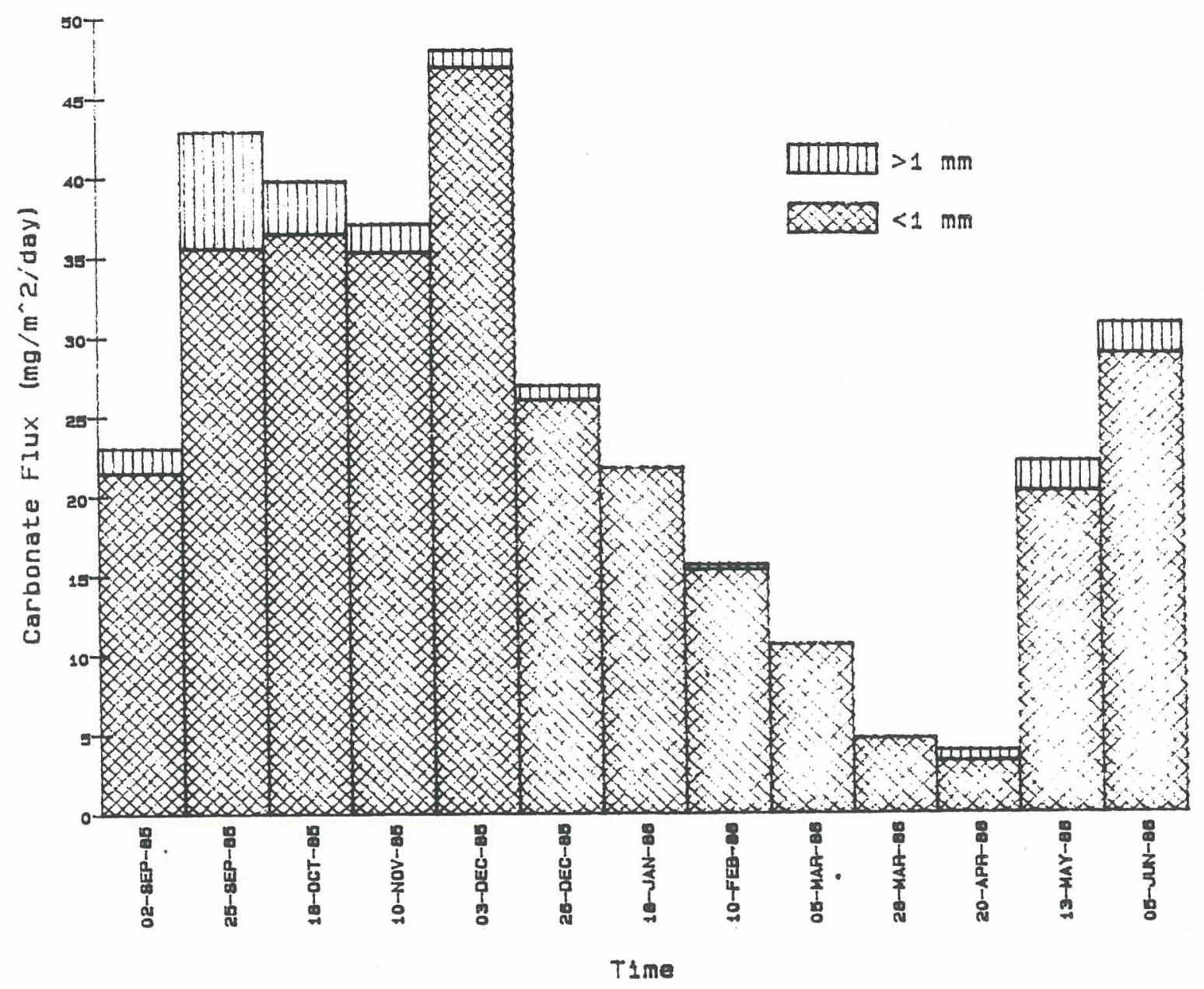

\begin{tabular}{|c|c|c|c|c|c|c|}
\hline $\begin{array}{l}\text { Sampla } \\
\text { I.D. }\end{array}$ & $\begin{array}{l}\text { CRTA } \\
\qquad i\end{array}$ & $\begin{array}{l}\text { CRTA \% } \\
\text { tot. } 1\end{array}$ & $\begin{array}{l}\text { ORTA } \\
+1\end{array}$ & $\begin{array}{l}\text { CRTA } \% \\
\text { tot. }\end{array}$ & $\begin{array}{l}\text { CRTA } \\
\text { totä }\end{array}$ & $\begin{array}{l}\text { CRTA } \\
\text { total }\end{array}$ \\
\hline 57 NA:-3058-1 & 21.54 & 39.97 & 1.55 & 2.88 & 23.89 & 42.65 \\
\hline 58 n+1-3058-2 & 35.54 & 51.08 & 7.37 & 10.72 & $42.9 i$ & $\mathrm{E}_{2 .} .4 \mathrm{2}$ \\
\hline 69 NA1-3058-3 & 36.47 & 52.93 & 3.37 & 4.93 & 35.81 & 57.98 \\
\hline $60 \mathrm{NA}:-3853-4$ & 35.29 & 51.97 & 1.80 & 2.55 & 37.29 & 54.62 \\
\hline$E: N A 1-3058 \times$ & 45.88 & 51.07 & 1.05 & 1.19 & 47.97 & 52.28 \\
\hline$Q 2 M A 1-3059-6$ & 25.00 & 47.67 & 0.91 & 1.67 & 28.91 & 48.34 \\
\hline $63 \mathrm{MA1}-3258-7$ & 21.75 & 47.88 & 0.02 & B. .04 & 21.70 & $4: 50$ \\
\hline 64 NA $-3058-8$ & 15.31 & 50.80 & 0.34 & 1.19 & 15.05 & 54.67 \\
\hline 65 HA - $3053-9$ & 10.52 & 49.09 & 0.00 & 0.00 & 10.62 & 49.00 \\
\hline EE NA $1-3058-10$ & 4.70 & 57.35 & 0.00 & 2.00 & 4.70 & 67.26 \\
\hline 57 19:-3058-11 & 3.25 & 51.12 & 0.68 & $10.6 E$ & 3.94 & 61.75 \\
\hline $82+4:-3052-12$ & 20.23 & 45.93 & 1.89 & 4.35 & 22.11 & $5: \cdot 9$ \\
\hline Es MA:-205e-1J & 28.81 & 48.51 & 1.95 & 3.29 & 30.75 & $51.9 \%$ \\
\hline
\end{tabular}




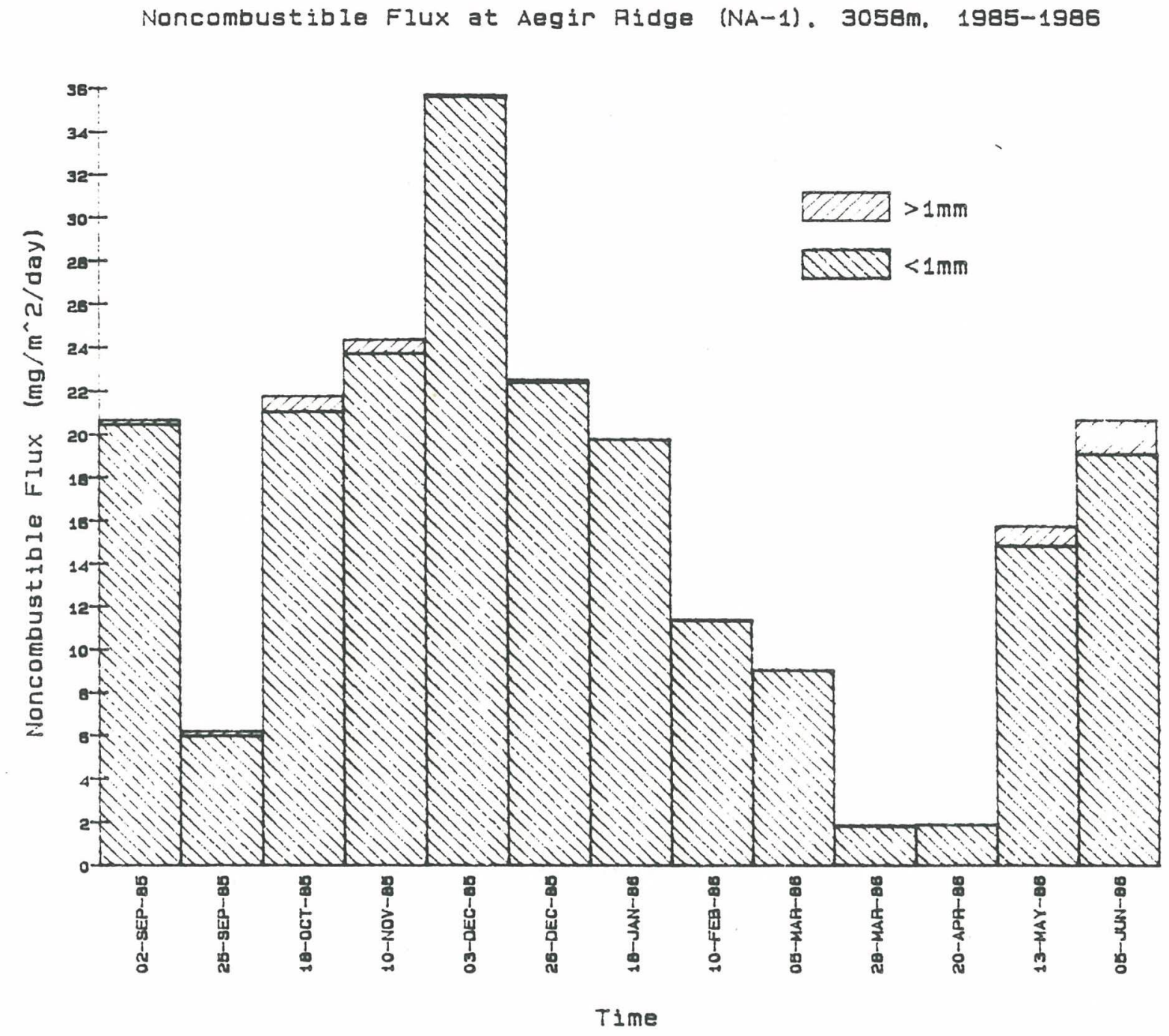

\begin{tabular}{|c|c|c|c|c|c|c|}
\hline $\begin{array}{l}\text { Samfle } \\
\text { ID\# }\end{array}$ & $\begin{array}{l}\text { NONC } \\
<1\end{array}$ & $\begin{array}{l}\text { NDNC } \% \\
\text { tot. } i\end{array}$ & $\begin{array}{l}\text { NONC } \\
>1\end{array}$ & $\begin{array}{l}\text { NONC \% } \\
\text { tot. } \% 1\end{array}$ & $\begin{array}{l}\text { NONC } \\
\text { total }\end{array}$ & $\begin{array}{l}\text { NONE \% } \\
\text { total }\end{array}$ \\
\hline 57 NA1-305E-1 & 20.46 & 37.96 & 0.24 & 0.45 & 20.70 & 38.42 \\
\hline $58 \quad N A 1-3058-2$ & 5.99 & 8.71 & 0.25 & 0.37 & 6.24 & 9.08 \\
\hline 59 NAI $-3058-3$ & 21.05 & 30.58 & 0.73 & 1.06 & 21.79 & 31.65 \\
\hline $50 \mathrm{NA1}-3058-4$ & 23.74 & 34.95 & 0.53 & 2.93 & 24.38 & 35.90 \\
\hline 51 NA1-305E-5 & 35.60 & 38.78 & 0.11 & 0.12 & 35.71 & 28.91 \\
\hline 62 NA: $-3058-6$ & 22.42 & 41.10 & 0.13 & 0.24 & 22.55 & 41.33 \\
\hline $63 N A 1-3058-7$ & 15.74 & 43.41 & 0.00 & 0.01 & 19.7 .4 & 43.42 \\
\hline 54 NA1-3058-8 & 11.34 & 39.77 & 0.03 & 0.10 & 11.37 & 39.87 \\
\hline 65 NAI-3058-5 & 9.04 & 41.50 & 0.02 & 0.07 & 9.05 & 41.87 \\
\hline 66 NAI-3058-10 & 1.84 & 26.34 & 0.04 & 0.50 & $1.8 ?$ & 25.84 \\
\hline 57 NA1-3058-1! & 1.91 & 29.51 & 0.01 & 0.23 & 1.92 & 30.14 \\
\hline $58 N A 1-3055-12$ & $\{4.5 i$ & 34.28 & 9.92 & 2.13 & 15.73 & $3 E .41$ \\
\hline 59 NAI $-3058-13$ & 19.05 & 32.15 & 1.60 & 2.70 & $20.5 E$ & $34 . B E$ \\
\hline
\end{tabular}

Flux is in mo/mnztday. 
Combustible Flux at Aegir Ridge (NA-1). 3058m, 1985-86

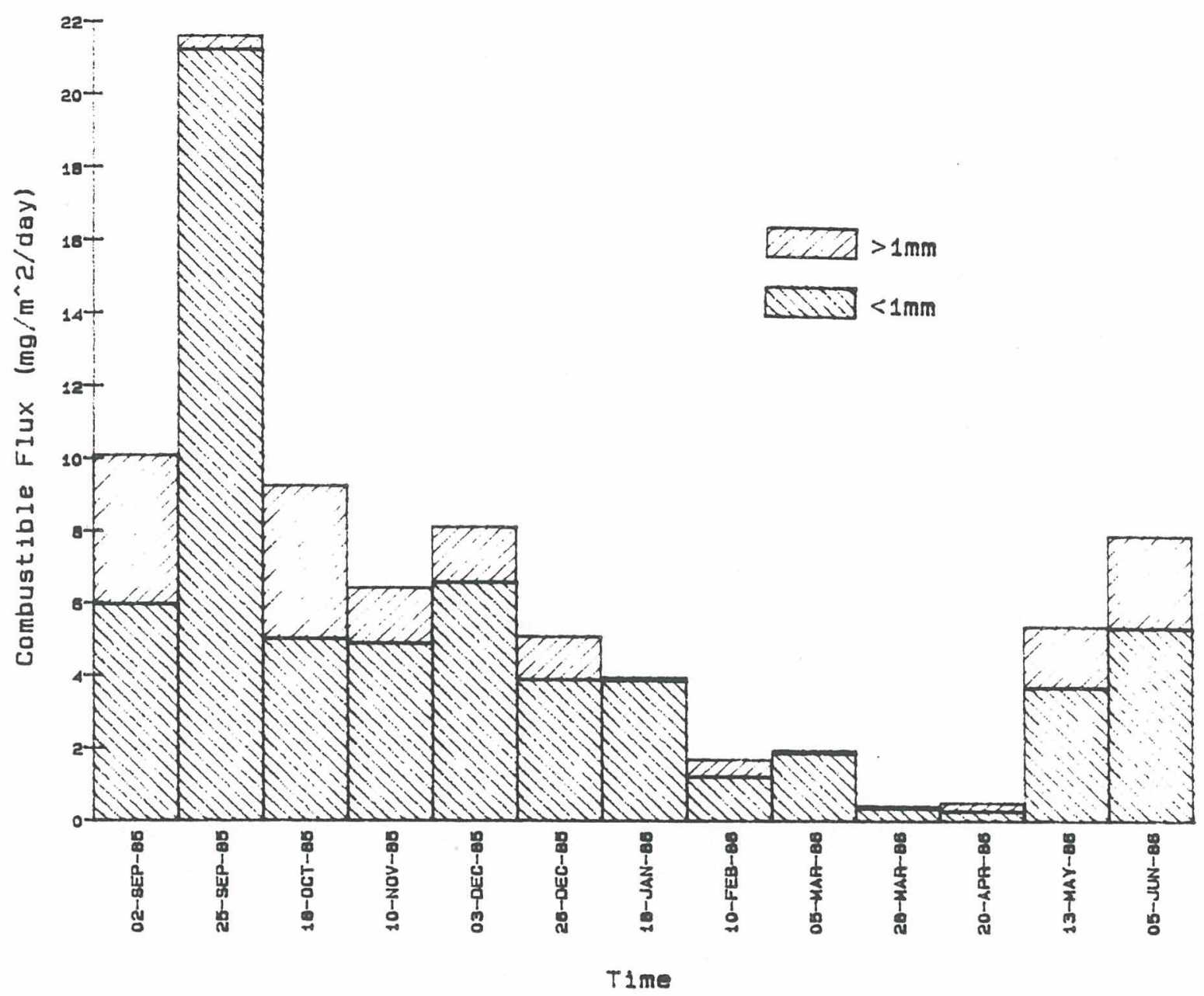

\begin{tabular}{|c|c|c|c|c|c|c|}
\hline $\begin{array}{l}\text { Sample } \\
\text { ID\# }\end{array}$ & $\begin{array}{l}\text { COMB } \\
<1\end{array}$ & $\begin{array}{l}\text { COMB \% } \\
\text { tot. }<1\end{array}$ & $\begin{array}{l}\text { COMB } \\
>1\end{array}$ & $\begin{array}{l}\text { COMB } \% \\
\text { tot. }>1\end{array}$ & $\begin{array}{l}\text { COMB } \\
\text { TOTAL }\end{array}$ & $\begin{array}{l}\text { COMB \% } \\
\text { total }\end{array}$ \\
\hline 57 NA1-3058-1 & 5.97 & 11.08 & 4.12 & 7.64 & 10.09 & 18.72 \\
\hline $58 N A 1-3058-2$ & 21.23 & 30.88 & 0.39 & 0.56 & 21.62 & 31.44 \\
\hline 59 NA1-3058-3 & 5.04 & 7.31 & 4.22 & 6.13 & 9.25 & 13.44 \\
\hline 60 NA1-3058-4 & 4.90 & 7.21 & 1.54 & 2.26 & 6.43 & 9.47 \\
\hline $51 N A 1-3058-5$ & 6.58 & 7.17 & $1.5 \overline{3}$ & 1.66 & 8.11 & 8.83 \\
\hline E2 NA1-3058-5 & 3.89 & 7.14 & 1.19 & 2.18 & 5.08 & 9.32 \\
\hline $63 \mathrm{NA} 1-3058-7$ & 3.87 & 8.52 & 0.08 & 0.17 & 3.95 & 8.68 \\
\hline E4 NA1-3058-8 & 1.22 & 4.27 & 0.48 & 1.68 & 1.70 & 5.96 \\
\hline 65 NA1-3058-9 & 1.88 & 8.68 & 0.08 & 0.39 & 1.96 & 9.07 \\
\hline EE NA1-3058-10 & 0.35 & 5.05 & 0.05 & 0.79 & 0.41 & 5.84 \\
\hline $67 N A 1-3058-11$ & 0.27 & 4.27 & 0.24 & 3.69 & 0.51 & 7.97 \\
\hline $68 N A 1-3058-12$ & 3.68 & 8.52 & 1.68 & 3.89 & 5.36 & 12.40 \\
\hline $69 N A 1-3058-13$ & 5.31 & 8.96 & 2.54 & 4.28 & 7.85 & 13.25 \\
\hline
\end{tabular}

Flux is in $m g / m^{\prime} 2 /$ day. 
Biogenic Silica FIux at Aegir Ridge (NA-1), 3058m. 1985-86

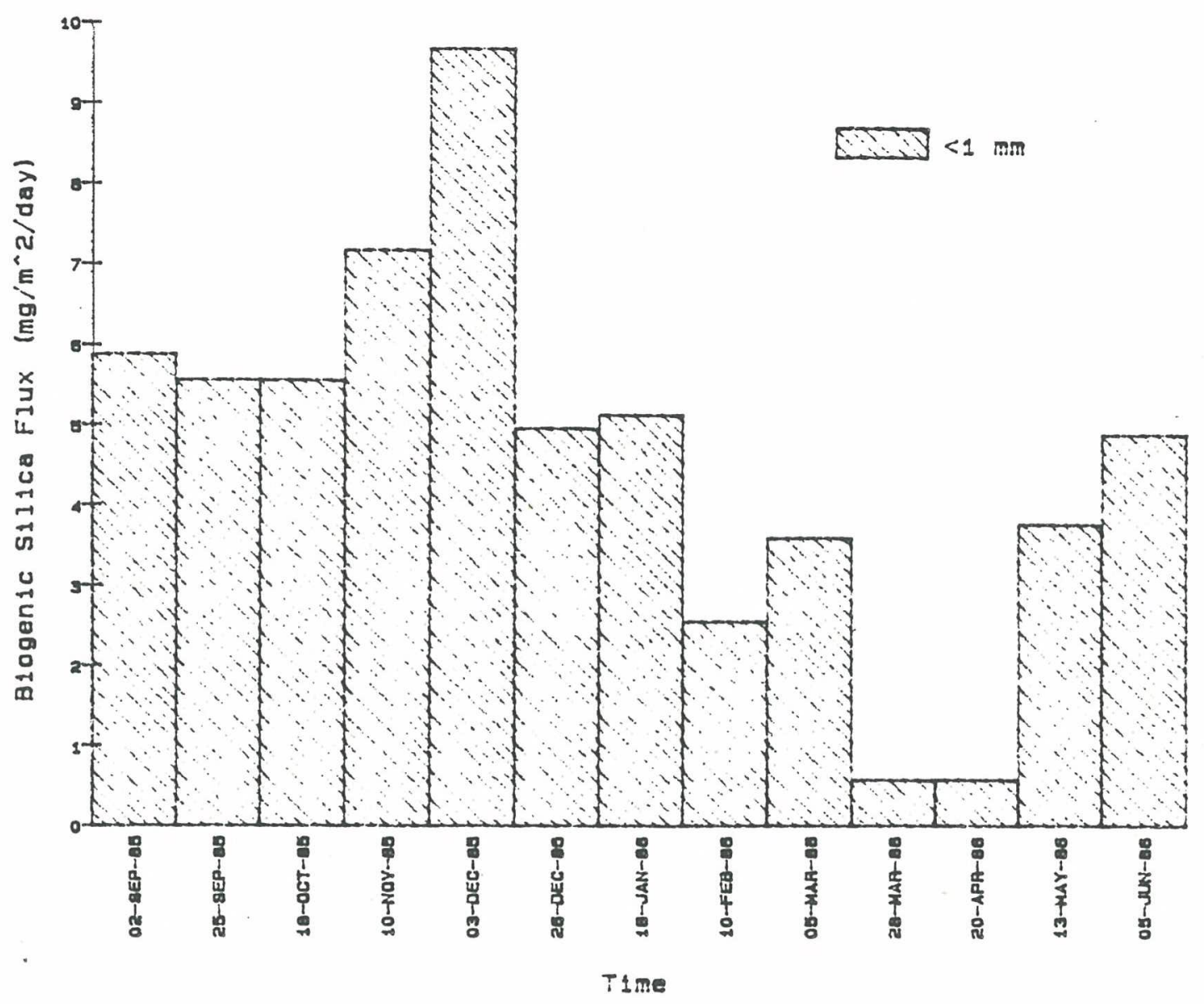

\begin{tabular}{|c|c|c|c|}
\hline $\begin{array}{l}\text { Sample } \\
\text { ID\# }\end{array}$ & $\begin{array}{l}\text { OPAL } \\
<1\end{array}$ & $\begin{array}{l}\text { OPAL \% } \\
\text { NCf. }<1\end{array}$ & $\begin{array}{l}\text { OPAL } \% \\
\text { tot. }<1\end{array}$ \\
\hline 57 NA1-3058-1 & 5.88 & 28.40 & 10.91 \\
\hline 58 NA1-3058-2 & 5.56 & 89.07 & 8.09 \\
\hline 59 NA1-3058-3 & 5.55 & 25.47 & 8.06 \\
\hline $50 N A 1-305 B-4$ & 7.17 & 29.41 & 10.56 \\
\hline $51 N A 1-3058-5$ & 9.67 & 27.08 & 10.53 \\
\hline $62 N A 1-3058-6$ & 4.95 & 21.96 & 9.08 \\
\hline E3 NA1-3058-7 & 5.12 & 25.93 & 11.26 \\
\hline $64 N A 1-3058-8$ & 2.55 & 22.42 & 8.94 \\
\hline E5 NAI-3058-9 & 3.59 & 39.63 & 16.59 \\
\hline EE NA1-3058-10 & 0.58 & 30.98 & 8.31 \\
\hline $67 N A 1-3058-11$ & 0.58 & 30.18 & 9.10 \\
\hline $68 N A 1-3058-12$ & 3.76 & 23.90 & 8.70 \\
\hline $69 N A 1-3058-13$ & 4.87 & 23.57 & 8.22 \\
\hline
\end{tabular}

Flux is in $m g / m^{\wedge} 2 / d a y$.

Not enough sample in $1 \mathrm{~mm}$ fraction to analyze for Opal.

"Nef." = "\% of noncombustible flux". 
Liticgenic Flux at Aegir Ridge (NA-1), $3058 \mathrm{~m}, 1985-6$

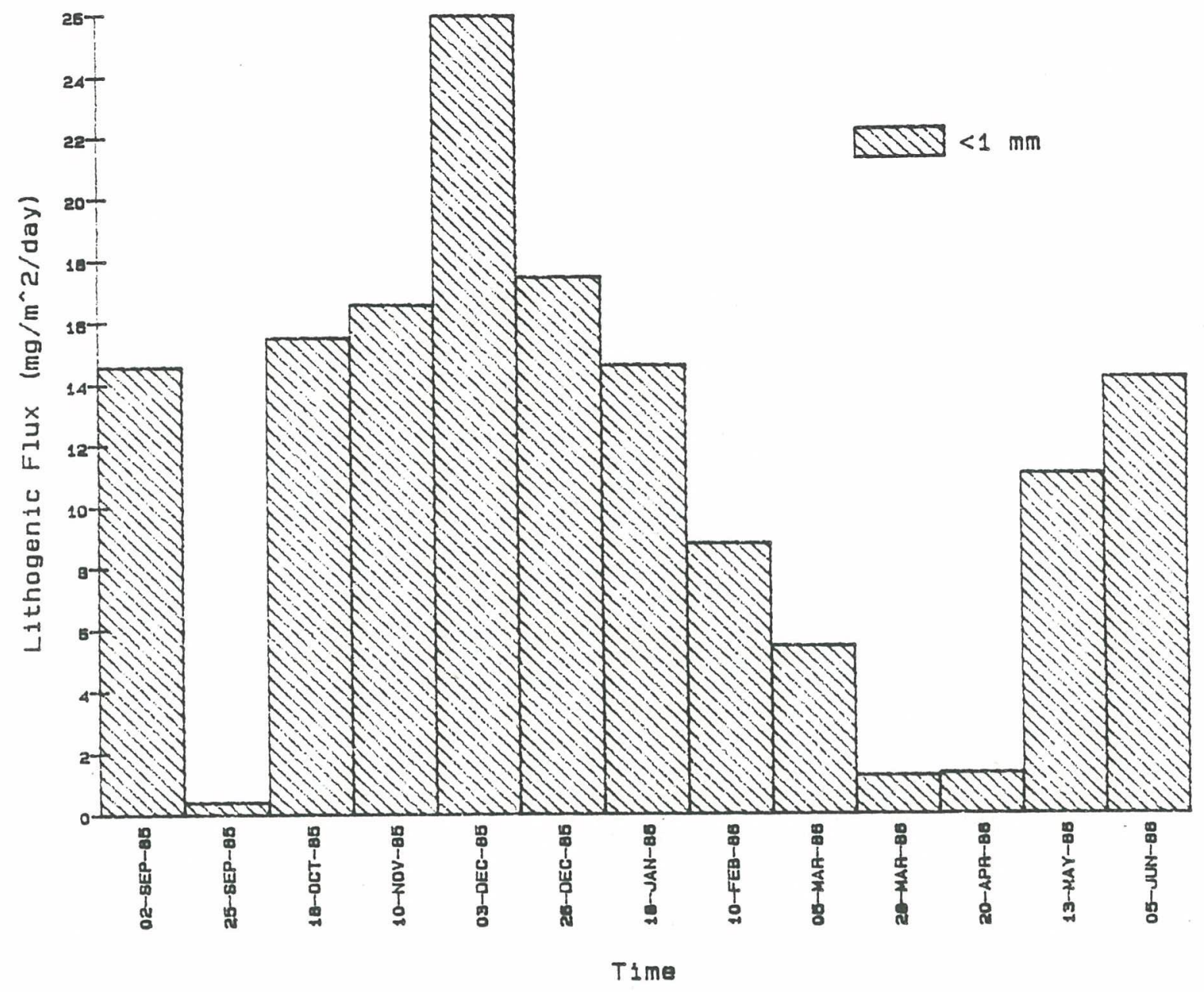

\begin{tabular}{|c|c|c|c|}
\hline $\begin{array}{l}\text { Sample } \\
\text { I.D. }\end{array}$ & $\begin{array}{l}\text { LITH } \\
<1\end{array}$ & $\begin{array}{l}\text { LITH<1 } \\
\% \text { NCMb. }\end{array}$ & $\begin{array}{l}\text { LITH<1 } \\
\% \text { tot. }\end{array}$ \\
\hline & & & \\
\hline 57 NA1-3058-1 & 14.58 & 70.42 & 27.05 \\
\hline $58 N A 1-3058-2$ & 0.43 & 6.86 & 0.62 \\
\hline $59 N A 1-3058-3$ & 15.50 & 71.16 & 22.52 \\
\hline $50 N A 1-3058-4$ & 16.57 & 67.99 & 24.41 \\
\hline B1 NA1-3058-5 & 25.93 & 72.61 & 28.25 \\
\hline E2 NA1-3058-5 & 17.47 & 77.47 & 32.02 \\
\hline $63 N A 1-305 B-7$ & 14.62 & 74.05 & 32.15 \\
\hline $64 N A 1-3058-3$ & 8.79 & 77.31 & 30.82 \\
\hline 65 NA1-3058-9 & 5.45 & 60.19 & 25.20 \\
\hline EE NA1-3058-10 & 1.26 & 67.15 & 18.02 \\
\hline $67 N A 1-3058-11$ & 1.33 & 69.07 & 20.82 \\
\hline 68 NA1-3058-12 & 11.05 & 70.25 & 25.58 \\
\hline EG NA1-3058-13 & 14.19 & 68.67 & 23.94 \\
\hline
\end{tabular}

Flux is in $m g / m^{\prime 2} /$ day.

$\%$ Ncmb. = "\% of noncombustible flux".

Not enough $1 \mathrm{~mm}$ fraction to do analysis. 
Carbon Flux at Aegir Ridge (NA-1), 3058m, 1985-86

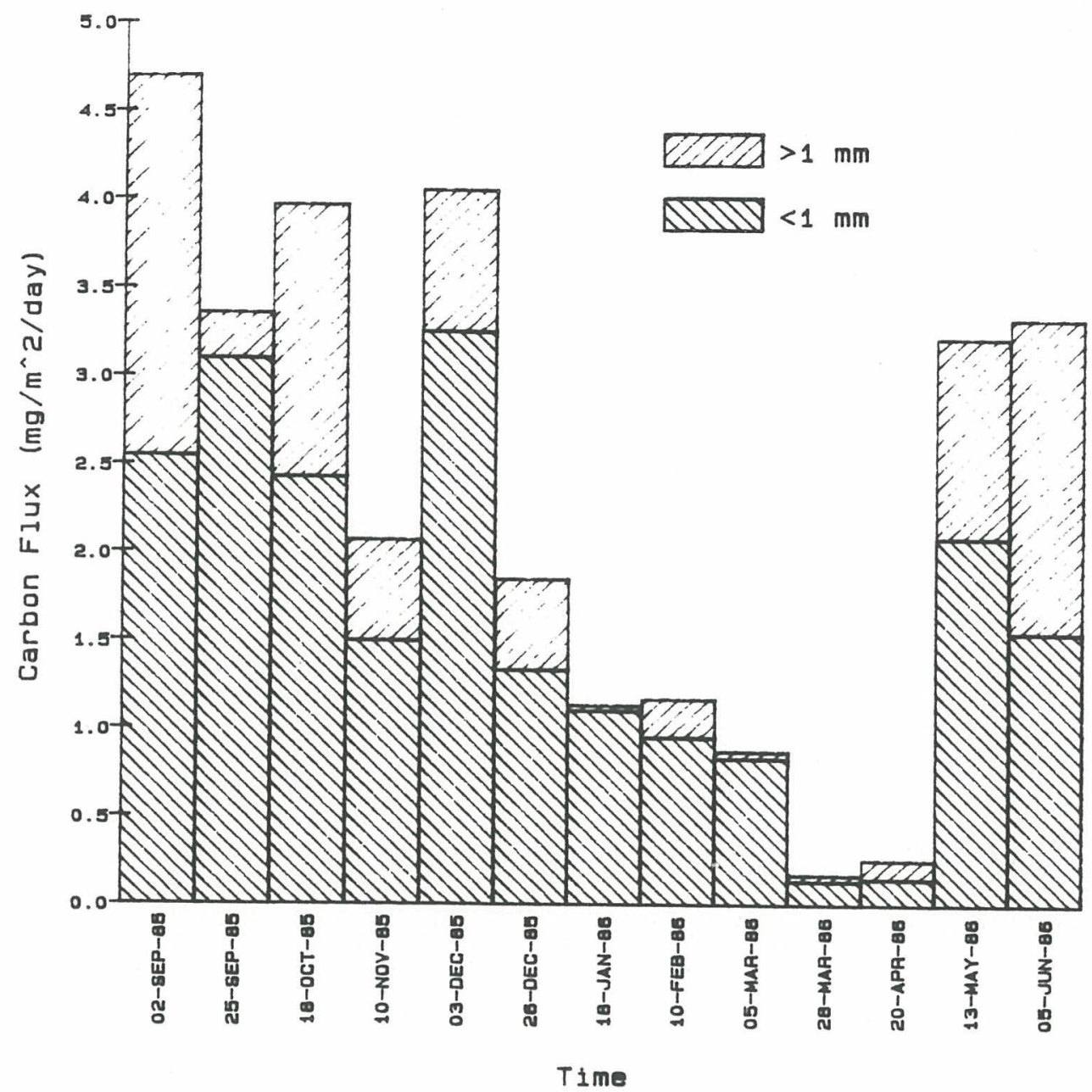

\begin{tabular}{|c|c|c|c|c|c|c|}
\hline $\begin{array}{l}\text { Sample } \\
\text { I.D. }\end{array}$ & $\begin{array}{l}\text { CRNC } \\
\vdots 1\end{array}$ & $\begin{array}{l}\text { CRNC } 1 \\
\% \text { cmb } f .\end{array}$ & $\begin{array}{l}\text { CRNC } \\
>1\end{array}$ & $\begin{array}{l}\text { CRNC } 1 \\
\% \text { cmbf. }\end{array}$ & $\begin{array}{l}\text { CRNC } \\
\text { total }\end{array}$ & $\begin{array}{l}\text { CRNCtot. } \\
\text { \%cmbf. }\end{array}$ \\
\hline 57 NA1-3058-1 & 2.55 & 25.25 & 2.16 & 21.36 & 4.70 & $4 E .62$ \\
\hline 58 NA1-3058-2 & 3.10 & 14.33 & 0.26 & 1.19 & 3.36 & 15.52 \\
\hline $59 \mathrm{NA1}-3058-3$ & 2.43 & 26.28 & 1.54 & 16.62 & 3.97 & 42.90 \\
\hline $50 N A_{1}-3058-4$ & 1.50 & 23.37 & 0.58 & 8.97 & 2.08 & 32.34 \\
\hline 61 NA1-3058-5 & 3.26 & 40.17 & 0.80 & 9.90 & 4.06 & 50.06 \\
\hline 62 NAI-3058-6 & 1.34 & 26.29 & 0.52 & 10.25 & 1.86 & 36.54 \\
\hline $63 N A 1-3058-7$ & 1.10 & 27.99 & 0.04 & 0.89 & 1.14 & 28.88 \\
\hline $54 N A 1-3058-8$ & 0.95 & 56.04 & 0.22 & 13.16 & 1.18 & 69.21 \\
\hline $65 N A 1-3058-9$ & 0.84 & 42.58 & 0.04 & 2.23 & 0.88 & 44.81 \\
\hline $66 \quad N A 1-3058-10$ & 0.14 & 33.53 & 0.04 & 9.68 & 0.18 & 43.21 \\
\hline $67 \mathrm{NA1}-3058-11$ & 0.15 & 29.44 & 0.11 & 21.58 & 0.26 & 51.02 \\
\hline $68 N A 1-3058-12$ & 2.09 & 39.02 & 1.14 & 21.27 & 3.23 & 60.3 \\
\hline $69 N A 1-3058-13$ & 1.55 & 19.77 & 1.79 & 22.74 & 3.34 & 42.5 \\
\hline
\end{tabular}

Flux is in mgim"z/day.

"combf" = "\% of combustible flux" 
Nițrogen Flux at Aegir Ridge (NA-1), 3058m, 1985-86

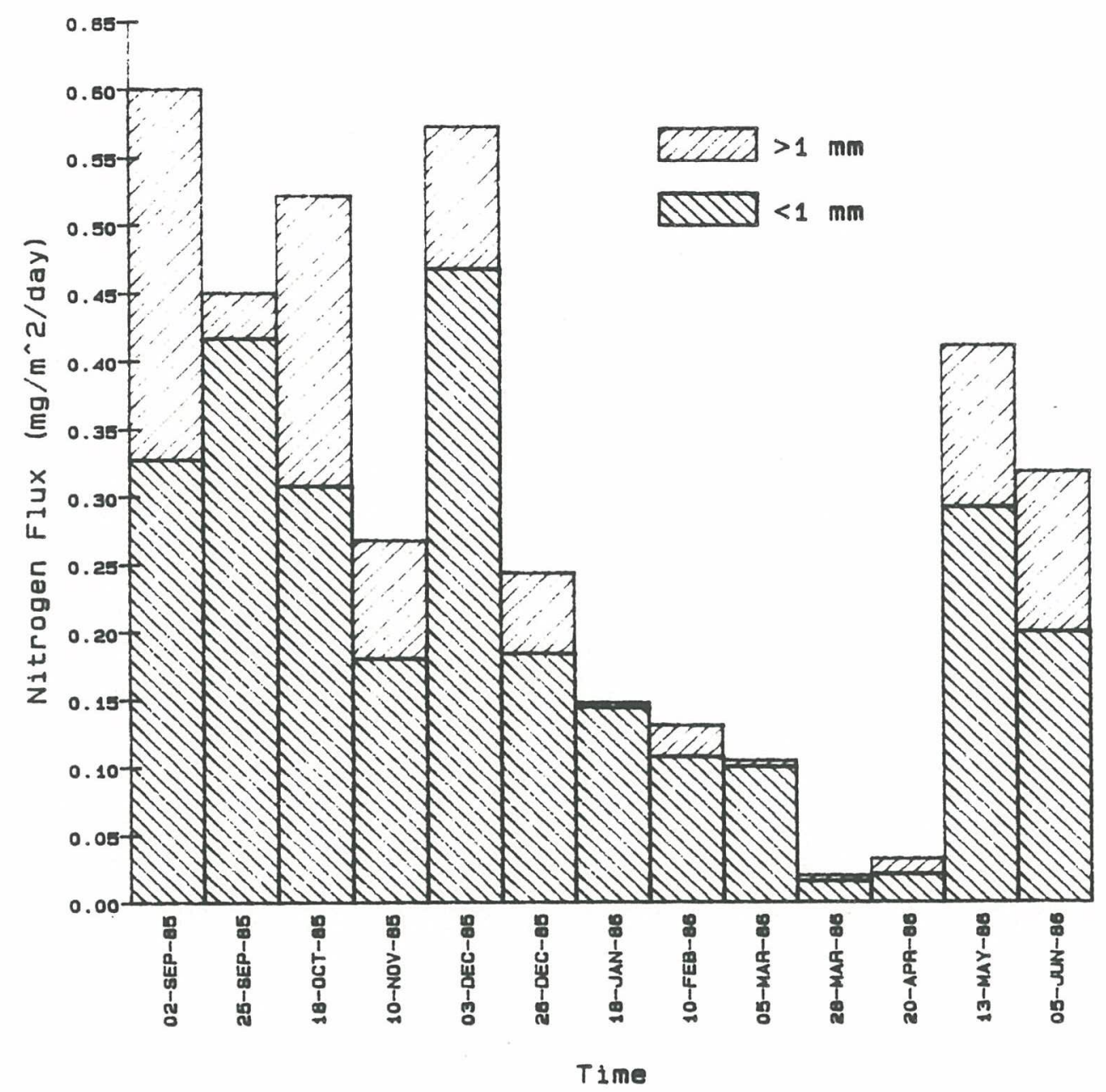

\begin{tabular}{|c|c|c|c|c|c|c|}
\hline $\begin{array}{l}\text { Sample } \\
\text { I.D. }\end{array}$ & $\begin{array}{l}\text { NTGN } \\
<1\end{array}$ & $\begin{array}{l}\text { NTENく } 1 \\
\% \text { cmb } f .\end{array}$ & $\begin{array}{l}\text { NTGN } \\
>1\end{array}$ & $\begin{array}{l}\text { NTGN> } 1 \\
\% \text { cmb } f .\end{array}$ & $\begin{array}{l}\text { NTGN } \\
\text { total }\end{array}$ & $\begin{array}{l}\text { NTGNtat. } \\
\% \text { cmbf. }\end{array}$ \\
\hline $57 \mathrm{NA1}-3058-1$ & 0.33 & 3.25 & 0.27 & 2.71 & 0.60 & 5.95 \\
\hline $58 N A 1-3058-2$ & 0.42 & 1.93 & 0.03 & 0.16 & 0.45 & 2.08 \\
\hline $59 N A 1-3058-3$ & 0.31 & 3.35 & 0.21 & 2.32 & 0.52 & 5.64 \\
\hline 50 NAI-3058-4 & 0.18 & 2.80 & 0.09 & 1.37 & 0.27 & 4.17 \\
\hline $51 N A 1-3058-5$ & 0.47 & 5.78 & 0.11 & 1.30 & 0.57 & 7.07 \\
\hline 62 NA1-3058-6 & 0.18 & 3.62 & 0.06 & 1.18 & 0.24 & 4.81 \\
\hline E3 NA1-3058-? & 0.14 & 3.65 & 0.00 & 0.09 & 0.15 & 3.7 \\
\hline $64 N A 1-3058-8$ & 0.11 & 6.36 & 0.02 & 1.38 & 0.13 & 7.7 \\
\hline $65 N A 1-3058-9$ & 0.10 & 5.12 & 0.00 & 0.23 & 0.11 & 5.3 \\
\hline EE NA1-3058-10 & 0.02 & 3.98 & 0.00 & 1.02 & 0.02 & 4.5 \\
\hline $67 N A 1-3058-11$ & 0.02 & 4.12 & 0.01 & 2.27 & 0.03 & 6.3 \\
\hline E8 NA $1-3058-12$ & 0.29 & 5.45 & 0.12 & 2.24 & 0.41 & 7.8 \\
\hline 69 NA1-3058-13 & 0.20 & 2.55 & 0.12 & 1.51 & 0.32 & 4.0 \\
\hline
\end{tabular}

Flux is in $m g / m^{\prime 2} 2 /$ day.

"\%cmbf" = "\% of combustible flux." 
Hydrogen Flux at Aegir Aidge (NA-1), 3058m. 1985-86

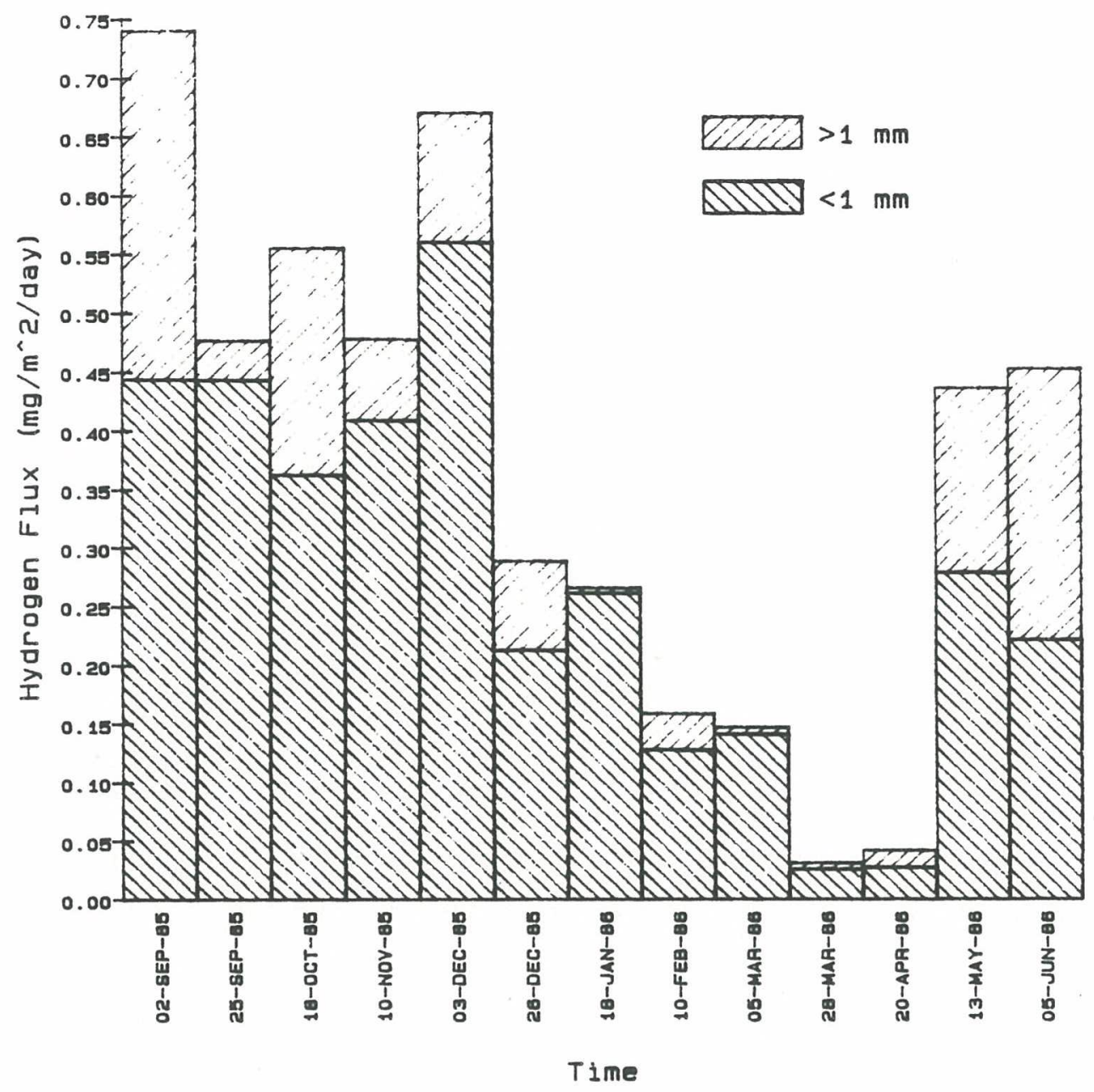

\begin{tabular}{|c|c|c|c|c|c|c|}
\hline $\begin{array}{l}\text { Sample } \\
\text { I.D. }\end{array}$ & $\begin{array}{l}\text { HYOC } \\
<1\end{array}$ & $\begin{array}{l}\text { HYOC } 1 \\
\% \text { cmbf. }\end{array}$ & $\begin{array}{l}\text { HYDC } \\
>1\end{array}$ & $\begin{array}{l}\text { HYOC } 1 \\
\% \text { cmbf. }\end{array}$ & $\begin{array}{l}\text { HYOC } \\
\text { total }\end{array}$ & $\begin{array}{l}\text { HYDCtot. } \\
\% \text { cmbf. }\end{array}$ \\
\hline 57 NA1-3058-1 & 0.44 & 4.40 & 0.30 & 2.94 & 0.74 & 7.3 \\
\hline $58 N A 1-3058-2$ & 0.44 & 2.05 & 0.03 & 0.15 & 0.48 & 2 \\
\hline $59 N A 1-3058-3$ & 0.36 & 3.92 & 0.19 & 2.09 & 0.56 & 5.6 \\
\hline E0 NA1-3058-4 & 0.41 & 6.37 & 0.07 & 1.08 & 0.48 & 7.4 \\
\hline E) NA1-3058-5 & 0.56 & 6.92 & 0.11 & 1.38 & 0.67 & 8.30 \\
\hline $62 N A 1-3058-6$ & 0.21 & 4.19 & 0.08 & 1.51 & 0.29 & 5.7 \\
\hline 53 NA1-3058-7 & 0.26 & 6.64 & 0.00 & 0.12 & 0.27 & 6.7 \\
\hline $64 N A 1-3058-8$ & 0.13 & 7.54 & 0.03 & 1.82 & 0.16 & 9.3 \\
\hline $65 \mathrm{NA1}-3058-9$ & 0.14 & 7.24 & 0.01 & 0.31 & 0.15 & 7.5 \\
\hline EG NA1-3058-10 & 0.03 & 6.50 & 0.01 & 1.34 & 0.03 & 7.8 \\
\hline $67 \mathrm{NA1}-3058-11$ & 0.03 & 5.45 & 0.02 & 2.99 & 0.04 & 8.4 \\
\hline $58 N A 1-3058-12$ & 0.28 & 5.21 & 0.16 & 2.94 & 0.44 & 8. \\
\hline E9 NA1-3058-13 & 0.22 & 2.82 & 0.23 & 2.95 & 0.45 & \\
\hline
\end{tabular}

Flux is in $m g / m$ "2/day.

"\%cmbf" = "\% of combustible flux" 


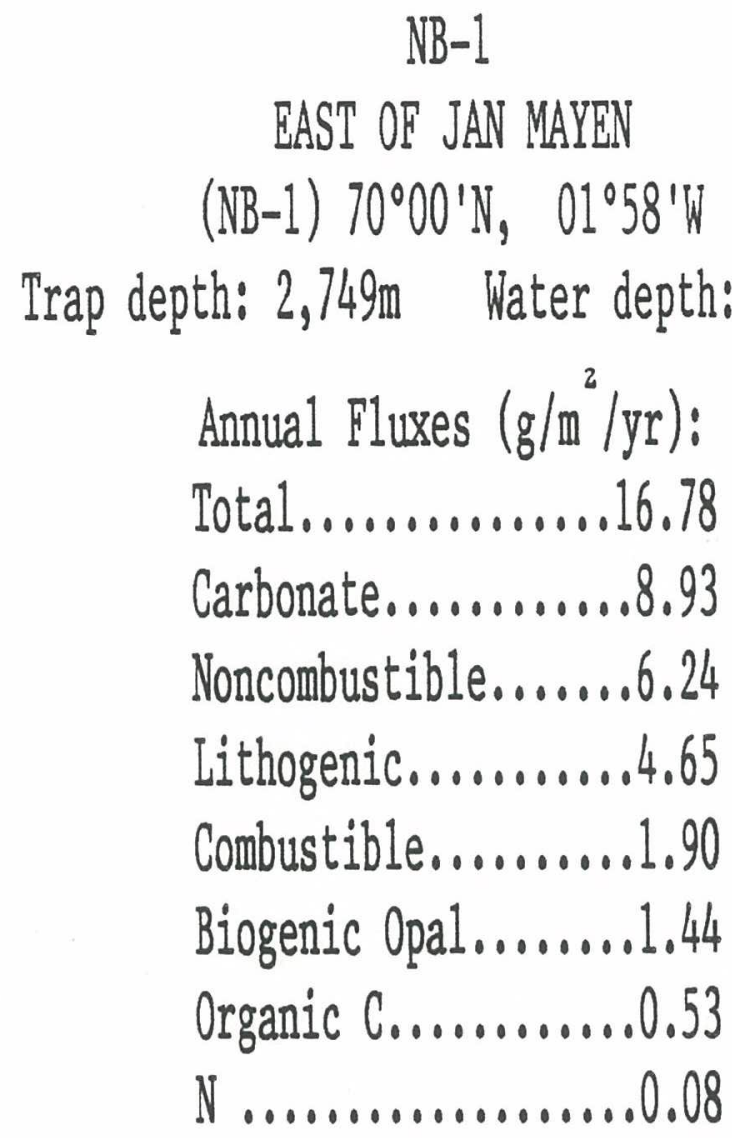

PARFLUX Mark 5-25

\begin{tabular}{|c|c|c|c|c|}
\hline $\begin{array}{l}\text { Sample } \\
\text { ID }\end{array}$ & $\begin{array}{l}\text { Dpening } \\
\text { Date }\end{array}$ & $\begin{array}{l}\text { Closing } \\
\text { Date }\end{array}$ & Span & $\begin{array}{l}\text { Mid. } \\
\text { Date }\end{array}$ \\
\hline 70 NB1-2815-1 & $20-A \cup G-85$ & $01-5 E P-85$ & 12 & $26-A \cup G-85$ \\
\hline 71 NB1-2815-2 & $01-5 E P-85$ & $13-5 E P-85$ & 12 & $07-S E P-85$ \\
\hline 72 NB1-2815-3 & $13-5 E P-85$ & $25-5 E P-85$ & 12 & $19-S E P-85$ \\
\hline 73 NB1-2815-4 & $25-5 E P-85$ & $07-0 C T-85$ & 12 & $01-0 C T-85$ \\
\hline 74 NB 1-2815-5 & $07-0 C T-85$ & $19-0 C T-85$ & 12 & $13-0 \mathrm{CT}-85$ \\
\hline 75 NB $1-2815-6$ & $19-0 C T-85$ & $31-0 C T-85$ & 12 & $25-0 \mathrm{CT}-85$ \\
\hline 76 NB1-2815-7 & $31-0 C T-85$ & $12-N O U-85$ & 12 & $05-N O U-85$ \\
\hline 77 NB $1-2815-8$ & $12-N O U-85$ & 24-NOU-85 & 12 & $18-N O U-85$ \\
\hline 78 NB1-2815-9 & $24-N O V-85$ & $\emptyset G-D E C-85$ & 12 & $30-N O U-85$ \\
\hline $79 N B 1-2815-10$ & $D E-D E C-85$ & $18-D E C-85$ & 12 & $12-D E C-85$ \\
\hline $80 N B 1-2815-11$ & $18-0 E C-85$ & $30-D E C-85$ & 12 & $24-D E C-85$ \\
\hline 81 NB $1-2815-12$ & $30-0 E C-85$ & $30-D E C-85$ & 12 & $05-J A N-86$ \\
\hline $82 N B 1-2815-13$ & $30-0 E C-85$ & $11-J A N-86$ & 12 & $05-J A N-86$ \\
\hline $83 N B 1-2815-14$ & $11-J A N-8 E$ & $23-J A N-86$ & 12 & $17-J A N-86$ \\
\hline $84 N B 1-2815-15$ & $23-J A N-86$ & $04-F E B-86$ & 12 & $29-J A N-86$ \\
\hline $85 N B 1-2815-16$ & $04-F E B-86$ & $1 E-F E B-8 E$ & 12 & $10-F E B-86$ \\
\hline 86 NB $1-2815-17$ & $16-F E B-86$ & $28-F E B-86$ & 12 & $22-F E B-86$ \\
\hline 87 NB1 $-2815-18$ & $28-F E B-8 E$ & $12-M A R-86$ & & $D E-M A R-8 E$ \\
\hline $88 N B 1-2815-19$ & $12-M A R-86$ & $24-M A R-86$ & & $18-M A R-86$ \\
\hline
\end{tabular}


Total flux at Jan Mayen (NB1), 2815m, 1985-1986

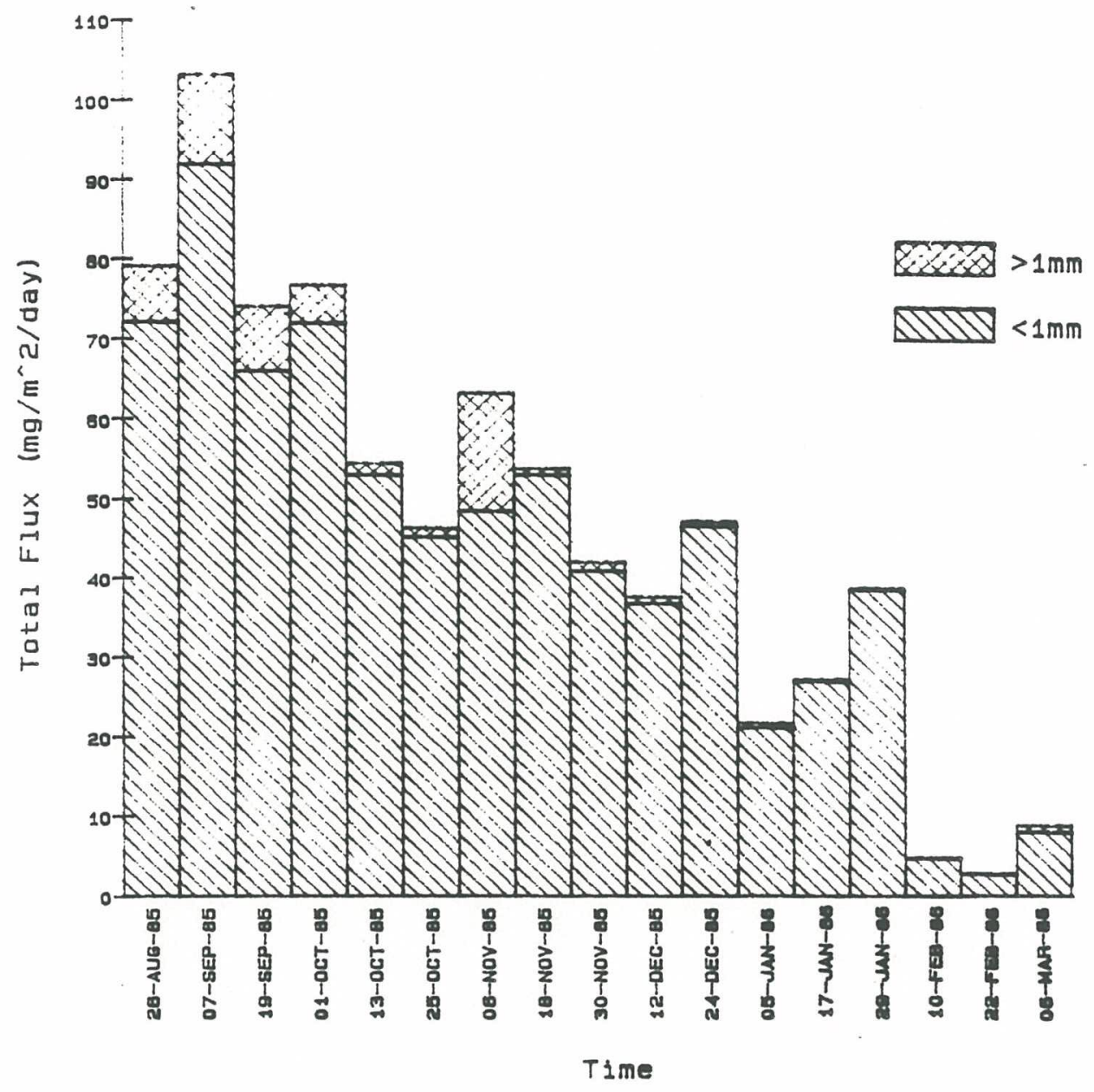

\begin{tabular}{|c|c|c|c|c|c|}
\hline $\begin{array}{l}\text { Sample } \\
\text { I.D. }\end{array}$ & $\begin{array}{l}\text { TTLF } \\
<1\end{array}$ & $\begin{array}{l}<1 \% \text { of } \\
\text { total }\end{array}$ & $\begin{array}{l}\text { TTLF } \\
>1\end{array}$ & $\begin{array}{l}1 \% \text { of } \\
\text { total }\end{array}$ & $\begin{array}{l}\text { TTLF } \\
\text { total }\end{array}$ \\
\hline $70 N B 1-2815-1$ & 72.19 & 91.14 & 7.01 & 8.86 & 79.21 \\
\hline 71 NB1-2815-2 & 91.93 & 89.06 & 11.29 & 10.94 & 103.22 \\
\hline $72 \mathrm{NB} 1-2815-3$ & 65.98 & 89.10 & 8.07 & 10.90 & 74.05 \\
\hline $73 \mathrm{NB} 1-2815-4$ & 71.94 & 93.86 & 4.71 & 6.14 & 76.65 \\
\hline $74 N B 1-2815-5$ & 52.90 & 97.22 & 1.51 & 2.78 & 54.41 \\
\hline 75 NB1-2815-6 & 45.13 & 97.55 & 1.13 & 2.45 & $46.2 E$ \\
\hline 76 NB 1-2815-7 & 48.36 & 76.51 & 14.85 & 23.49 & 63.22 \\
\hline 77 NB1-2815-8 & 52.95 & 98.41 & 0.85 & 1.59 & 53.80 \\
\hline 78 NB $1-2815-9$ & 40.77 & 97.33 & 1.12 & 2.67 & 41.89 \\
\hline $79 N B 1-2815-10$ & 36.70 & 97.73 & 0.85 & 2.27 & 37.55 \\
\hline $80 N B 1-2815-11$ & 46.52 & 98.56 & 0.68 & 1.44 & 47.20 \\
\hline 81 NB1-2815-12 & 21.13 & 97.30 & 0.59 & 2.70 & 21.72 \\
\hline 82 NB1-2815-13 & 26.87 & 98.79 & 0.33 & 1.21 & 27.20 \\
\hline 83 NB1-2815-14 & 38.43 & 99.13 & 0.34 & 0.87 & 38.76 \\
\hline 84 NB $1-2815-15$ & 4.70 & 98.10 & 0.09 & 1.90 & 4.79 \\
\hline $85 N B 1-2815-16$ & 2.72 & 97.75 & 0.06 & 2.25 & 2.79 \\
\hline 86 NB 1-2815-17 & 8.03 & 90.12 & 0.88 & 9.88 & 8.91 \\
\hline
\end{tabular}

Flux is in $m g / m " 2 / d a y$. 
Carbonate Flux at Jan Mayen (NB-1), 2815m, 1985-1986

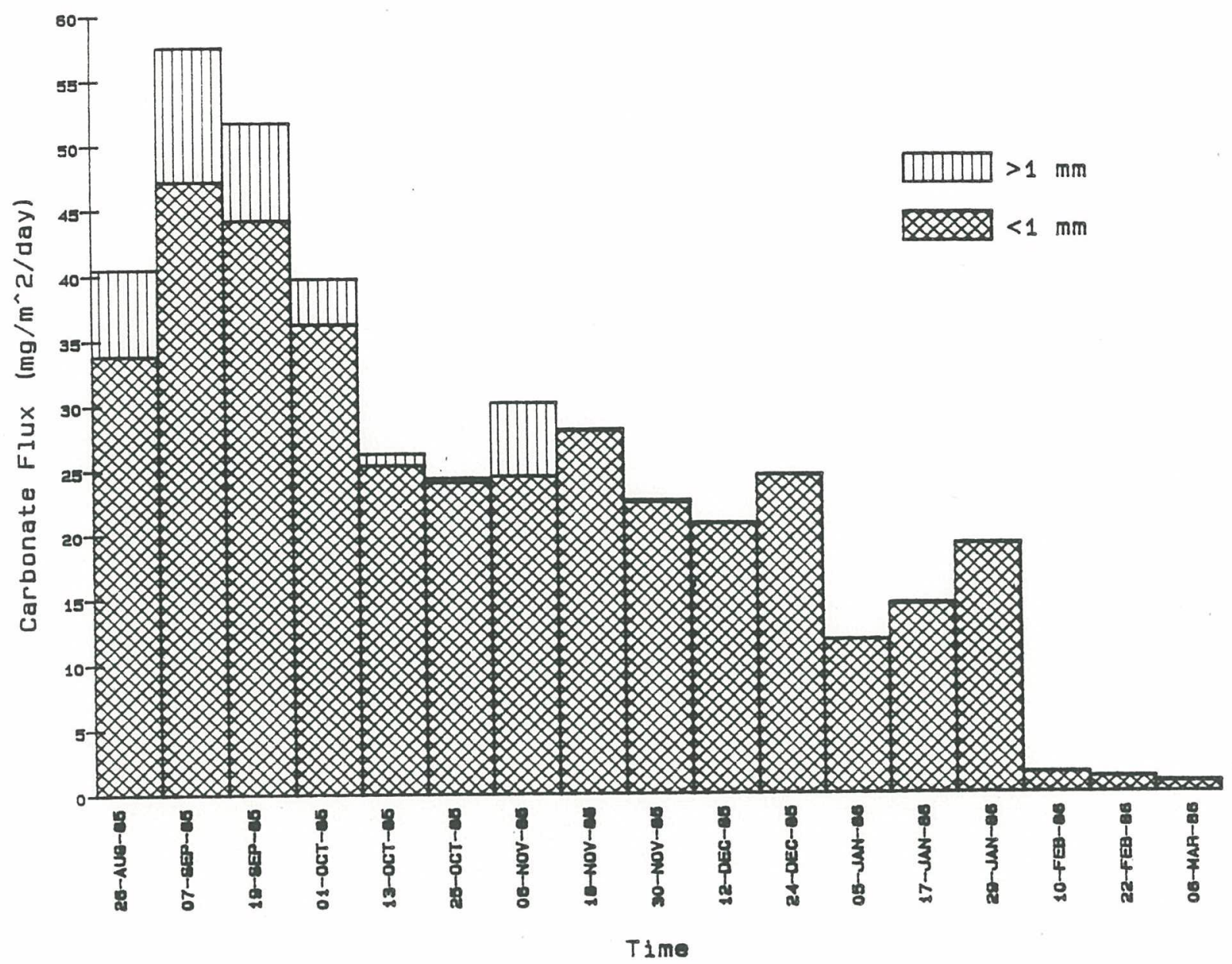

\begin{tabular}{|c|c|c|c|c|c|c|}
\hline $\begin{array}{l}\text { Sample } \\
\text { I.0. }\end{array}$ & $\begin{array}{l}\text { CRTA } \\
\vdots 1\end{array}$ & $\begin{array}{l}\text { CRTA } \% \\
\text { tot: }<1\end{array}$ & $\begin{array}{l}\text { CRTA } \\
>1\end{array}$ & $\begin{array}{l}\text { CRTA \% } \\
\text { tot. }>1\end{array}$ & $\begin{array}{l}\text { CRTA } \\
\text { total }\end{array}$ & $\begin{array}{l}\text { CRTA } \% \\
\text { total }\end{array}$ \\
\hline & & & & 84 & $40=5$ & [1 1 \\
\hline $\begin{array}{l}70 \text { NB1-2815-1 } \\
71 \text { NE1-2815-2 }\end{array}$ & 33.84 & 42.72 & 6.66 & 8.41 & $\begin{array}{l}46.56 \\
57.54\end{array}$ & 51.13 \\
\hline & 47.19 & 45.72 & 10.35 & 10.03 & 57.54 & 5. \\
\hline $\begin{array}{l}72 \text { NB1-2815-3 } \\
73 \text { NB1-2815-4 }\end{array}$ & 44.21 & 59.70 & 7.59 & 10.25 & 51.80 & 69.95 \\
\hline 73 NB 1-2815-4 & 36.25 & 47.29 & 3.49 & 4.55 & 39.74 & 51.85 \\
\hline 74 NB1-2815-5 & 25.33 & 46.55 & 0.89 & 1.64 & 26.22 & 48.19 \\
\hline 75 NB1-2815-E & 24.04 & 51.96 & 0.32 & $\oslash .69$ & 24.36 & 52.56 \\
\hline $76 \quad N B 1-2815-7$ & 24.50 & 38.76 & 5.67 & 8.97 & 30.17 & 47.72 \\
\hline 77 NB1-2815-8 & 28.01 & 52.06 & 0.13 & 0.24 & 28.14 & 52.30 \\
\hline 78 NB 1-2815-9 & 22.43 & 53.54 & 0.15 & 0.36 & 22.58 & 53.90 \\
\hline $79 \mathrm{NB} 1-2815-10$ & 20.74 & 55.24 & 0.07 & 0.19 & 20.31 & 55.42 \\
\hline $80 N B 1-2815-11$ & 24.52 & 51.95 & 0.07 & 0.15 & 24.59 & 52.10 \\
\hline $81 N B 1-2815-12$ & 11.80 & 54.34 & 0.12 & 0.00 & 11.80 & 54.34 \\
\hline 82 NB1-2815-13 & 14.59 & 53.64 & 0.10 & 0.37 & 14.69 & 54.00 \\
\hline $83 \mathrm{NB} 1-2815-14$ & 19.14 & 49.38 & 0.05 & 0.13 & 19.19 & 49.51 \\
\hline 84 NB1-2815-15 & 1.55 & 32.38 & 0.06 & 1.25 & 1.61 & 33.64 \\
\hline 85 NB1-2815-16 & 1.23 & 44.14 & 0.04 & 1.44 & 1.27 & 45.58 \\
\hline 86 NB1-2815-17 & 0.80 & 9.01 & 0.14 & 1.57 & 0.94 & 10.54 \\
\hline
\end{tabular}

Flux is in mg/m"2/day. 
Noncombustible Flux at Jan Mayen (NB-1). 2815m, 1985-1986

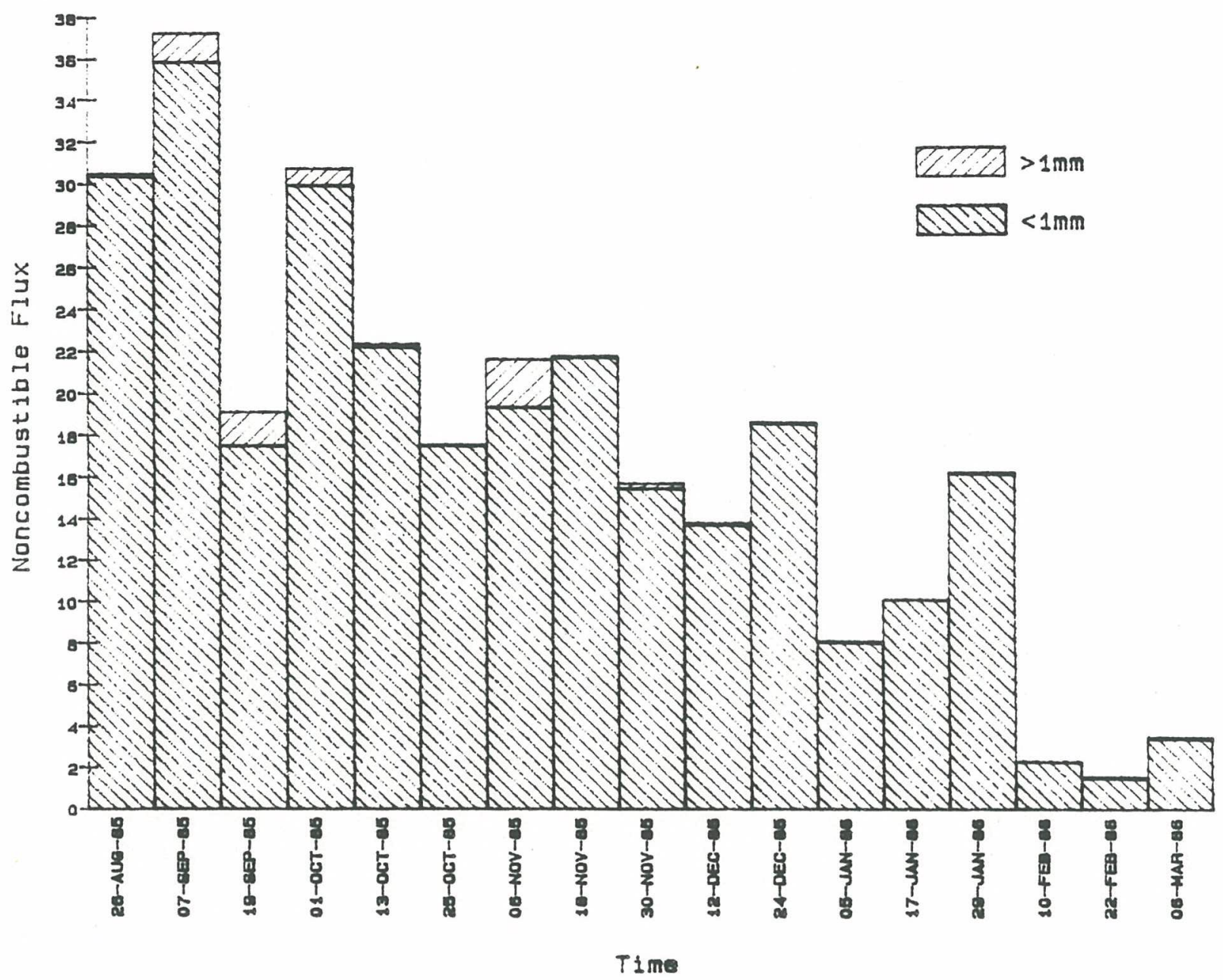

\begin{tabular}{|c|c|c|c|c|c|c|}
\hline $\begin{array}{l}\text { Sample } \\
\text { ID\# }\end{array}$ & $\begin{array}{l}\text { NONC } \\
\vdots 1\end{array}$ & $\begin{array}{l}\text { NONC } \% \\
\text { tot. } 1\end{array}$ & $\begin{array}{l}\text { NONC } \\
>1\end{array}$ & $\begin{array}{l}\text { NONC } \% \\
\text { tot. }>1\end{array}$ & $\begin{array}{l}\text { NONC } \\
\text { total }\end{array}$ & $\begin{array}{l}\text { NONC } \% \\
\text { total }\end{array}$ \\
\hline 70 NE1-2815-1 & 30.37 & 38.35 & 0.14 & 0.17 & 30.51 & 38.52 \\
\hline 71 NE1-2815-2 & 35.84 & 34.72 & 1.42 & 1.37 & 37.25 & JE. 09 \\
\hline 72 NB1-2815-3 & 17.49 & 23.61 & 1.50 & 2.16 & 19.09 & 25.77 \\
\hline 73 NB $1-2815-4$ & 29.94 & 39.07 & 0.81 & 1.06 & 30.75 & 40.12 \\
\hline 74 NB1-2815-5 & 22.22 & 40.84 & 0.18 & 0.34 & 22.40 & 41.18 \\
\hline 75 NB1-2815-6 & 17.48 & 37.79 & 0.03 & 0.05 & 17.51 & 37.85 \\
\hline 76 NE 1-2815-7 & 19.30 & 30.53 & 2.34 & 3.71 & 21.65 & 34.24 \\
\hline 77 NB $1-2815-8$ & 21.70 & 40.33 & 0.07 & 0.13 & 21.77 & 40.46 \\
\hline 78 NB $1-2815-9$ & 15.42 & 36.82 & 0.24 & 0.57 & 15.66 & 37.38 \\
\hline 79 NB1-2815-10 & 13.68 & 36.43 & 0.09 & 0.24 & 13.77 & $3 E .67$ \\
\hline $80 N B !-2815-11$ & 18.57 & 39.34 & 0.05 & 0.10 & 18.61 & 39.44 \\
\hline $81 \mathrm{NB} 1-2815-12$ & 8.05 & 37.08 & 0.05 & 0.23 & 8.10 & 37.31 \\
\hline $82 \mathrm{NB} 1-2815-13$ & 10.13 & 37.24 & 0.01 & 0.04 & 10.14 & 37.28 \\
\hline $83 \mathrm{NB} 1-2815-14$ & 16.17 & 41.70 & 0.02 & 0.06 & 16.19 & 41.76 \\
\hline $84 \mathrm{NB} 1-2815-15$ & 2.31 & 48.17 & 0.00 & 0.02 & 2.31 & 48.19 \\
\hline 85 NB1-2815-16 & 1.49 & 53.48 & 0.03 & 1.21 & 1.52 & 54.63 \\
\hline 86 NB1-2815-17 & 3.43 & 38.43 & 0.04 & 0.49 & 3.47 & 38.92 \\
\hline
\end{tabular}

Flux is in mgima/day. 
Combustible Flux at Jan Mayen (NB-1), 2815m, 1985-86

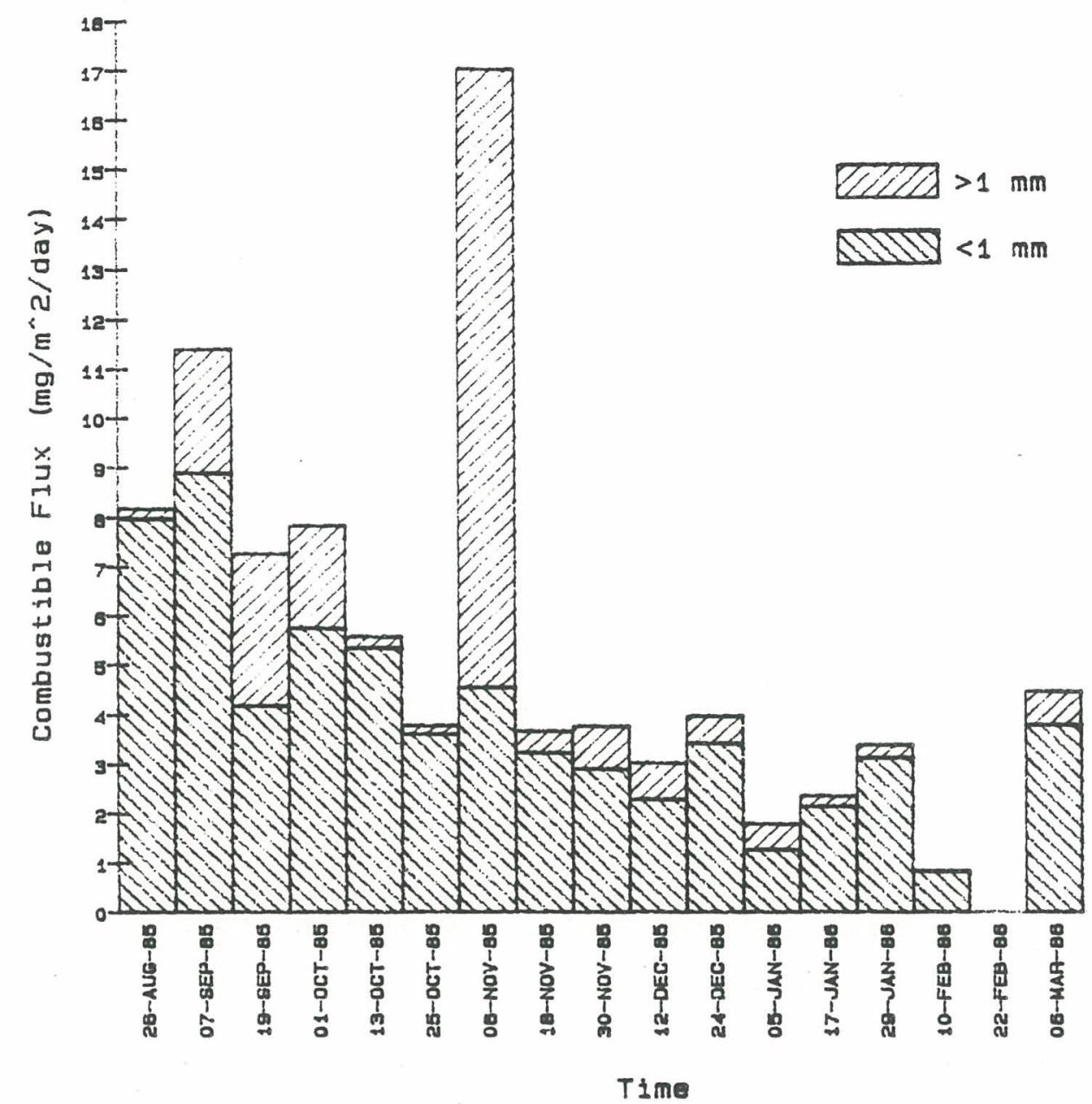

\begin{tabular}{|c|c|c|c|c|c|c|}
\hline $\begin{array}{l}\text { Samp le } \\
\text { ID\# }\end{array}$ & $\begin{array}{l}\text { COMB } \\
<1\end{array}$ & $\begin{array}{l}\text { COMB } \% \\
\text { tot. }<1\end{array}$ & $\begin{array}{l}\text { COMB } \\
>1\end{array}$ & $\begin{array}{l}\text { COMB } \% \\
\text { tot. } 1\end{array}$ & $\begin{array}{l}\text { COMB } \\
\text { TOTAL }\end{array}$ & $\begin{array}{l}\text { COME }: \\
\text { total }\end{array}$ \\
\hline & & & & & & \\
\hline 70 NB1-2815-1 & 7.98 & 10.07 & 0.21 & 0.27 & 8.19 & 10.34 \\
\hline 71 NB1-2815-2 & 8.90 & 8.63 & 2.50 & 2.43 & 11.41 & 11.05 \\
\hline 72 NE1-2815-3 & 4.18 & 5.65 & 3.10 & 4.19 & 7.28 & 9.84 \\
\hline 73 NE1-2815-4 & 5.75 & 7.50 & 2.10 & 2.74 & 7.85 & 10.24 \\
\hline 74 NB1-2815-5 & 5.35 & 9.83 & 0.24 & 0.44 & 5.59 & 10.27 \\
\hline 75 NB1-2815-6 & 3.61 & 7.80 & 0.19 & 0.42 & 3.80 & 8.22 \\
\hline 76 NB1-2815-7 & 4.56 & 7.21 & 12.49 & 19.75 & 17.04 & 26.96 \\
\hline 77 NB1-2815-8 & 3.24 & 6.03 & 0.44 & 0.82 & 3.68 & 6.84 \\
\hline 78 NE 1-2815-9 & 2.92 & 6.96 & 0.88 & 2.11 & 3.80 & 9.07 \\
\hline $79 N B 1-2815-10$ & 2.28 & 6.08 & 0.76 & 2.02 & 3.04 & 3.10 \\
\hline 80 NB $1-2815-11$ & 3.43 & 7.27 & 0.56 & 1.20 & 4.00 & 8.47 \\
\hline 81 NB1-2815-12 & 1.28 & 5.89 & 0.54 & 2.48 & 1.82 & 8.37 \\
\hline $82 N B 1-2815-13$ & 2.15 & 7.90 & 0.22 & 0.80 & 2.37 & 8.71 \\
\hline 83 NB $1-2815-14$ & 3.12 & 8.06 & 0.27 & 0.69 & 3.39. & 8.75 \\
\hline 84 NB1-2815-15 & 0.84 & 17.64 & 0.01 & 0.19 & 0.85 & 17.83 \\
\hline $85 N B 1-2815-16$ & 0.00 & 0.00 & 0.01 & 0.22 & 0.01 & 0.22 \\
\hline 86 NE1-2815-17 & 3.80 & 42.64 & 0.70 & 7.81 & 4.50 & 50.45 \\
\hline
\end{tabular}

Flux is in $m g / m^{\wedge} 2 / d a y$. 
Blogentc Silica Flux ot Jan Mayen (NB-1), 2815m, 1986

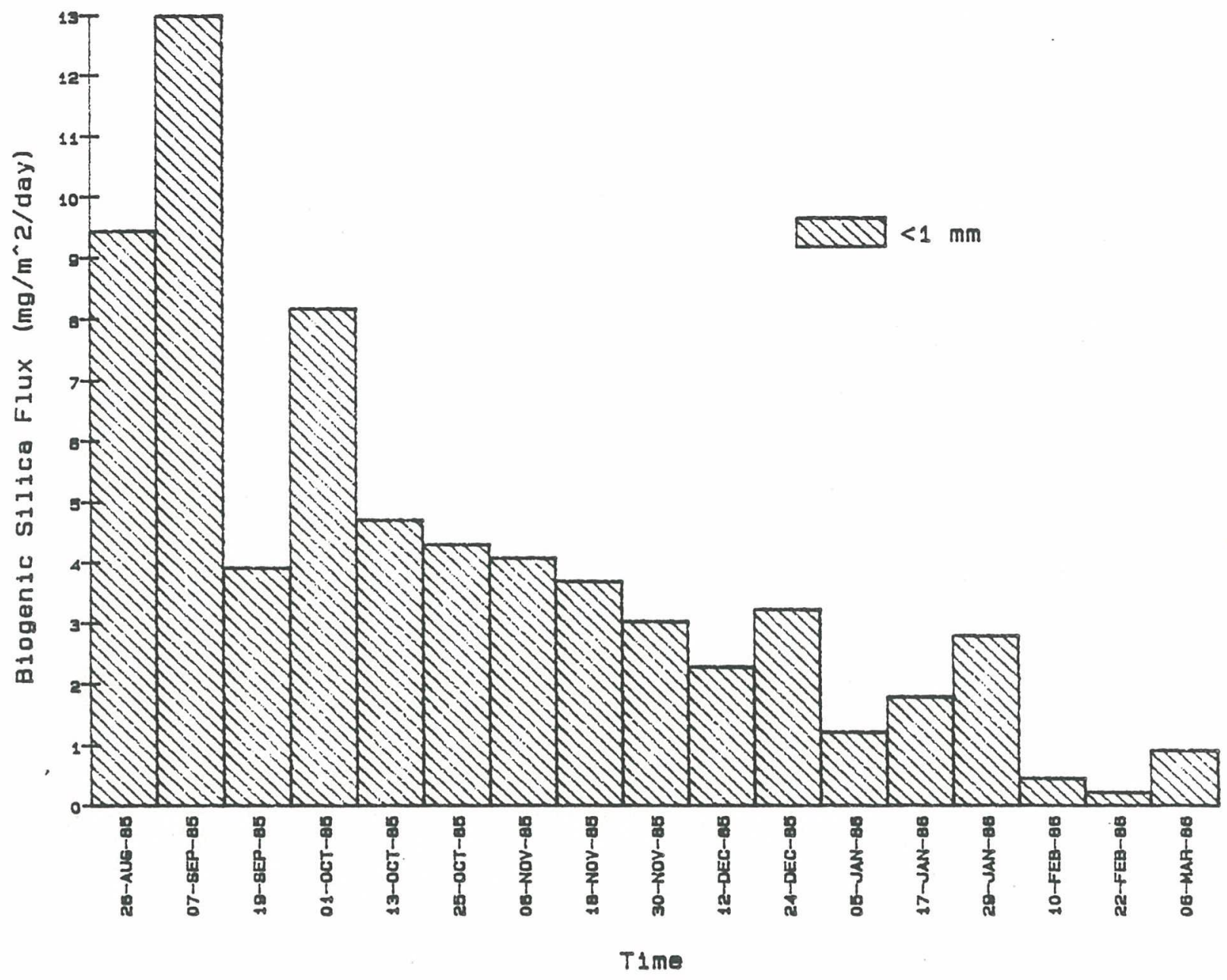

\begin{tabular}{|c|c|c|c|}
\hline $\begin{array}{l}\text { Sample } \\
\text { ID\# }\end{array}$ & $\begin{array}{l}\text { OPAL } \\
\vdots !\end{array}$ & $\begin{array}{l}\text { OPAL } \% \\
\text { NOF. } 1\end{array}$ & $\begin{array}{l}\text { OPAL } \% \\
\text { tot. } 1\end{array}$ \\
\hline 70 NB $1-2815-1$ & 9.44 & 30.94 & 11.92 \\
\hline 71 NB1-2815-2 & 12.99 & 34.87 & 12.58 \\
\hline 72 NB1-2815-3 & 3.91 & 20.49 & 5.28 \\
\hline 73 NB1-2815-4 & 8.17 & 26.57 & 10.66 \\
\hline $74 N B 1-2815-5$ & 4.70 & 20.98 & 8.64 \\
\hline 75 NB1-2815-6 & 4.30 & 24.56 & 9.2 \\
\hline 76 NB1-2815-7 & 4.08 & 18.85 & 6.4 \\
\hline 77 NB $1-2815-8$ & 3.69 & 16.95 & 6.8 \\
\hline $78 N B 1-2815-9$ & 3.03 & 19.35 & 7.2 \\
\hline $79 N B 1-2815-10$ & 2.28 & 16.56 & 6.0 \\
\hline $80 N B 1-2815-11$ & 3.23 & 17.35 & 5.8 \\
\hline 81 NB1-2815-12 & 1.22 & 15.06 & 5.6 \\
\hline $82 N B 1-2815-13$ & 1.79 & 17.67 & 6.5 \\
\hline $83 N B 1-2815-14$ & 2.80 & 17.32 & 7.2 \\
\hline $84 N B 1-2815-15$ & 0.46 & 19.91 & 9.6 \\
\hline $85 N B 1-2815-16$ & 0.22 & 14.77 & 7.8 \\
\hline $86 N B 1-2815-17$ & 0.92 & 26.82 & 10.3 \\
\hline
\end{tabular}

Flux is in $\mathrm{mg} / \mathrm{m}^{\prime \prime 2} / \mathrm{day}$.

Not enough sample in $1 \mathrm{~mm}$ fraction to analyze for Opal.

"Ncf." = "\% of noncombustible flux". 


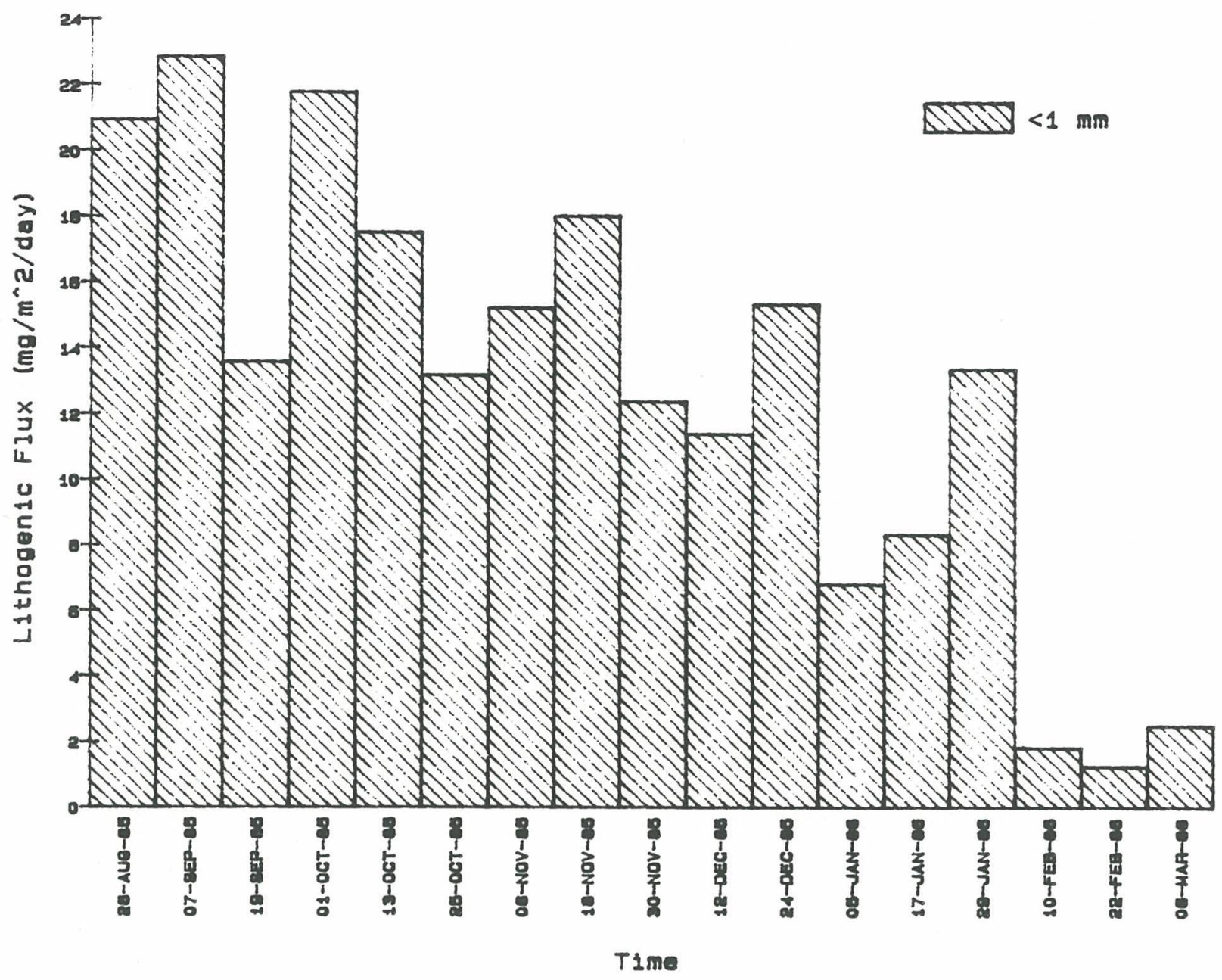

$\begin{array}{lrrr}\text { SAMPLE } & \text { LITH } & \text { LITHK1 } & \text { LITH I } \\ \text { I.D. } & \text { K1 } & \text { \%NCMb. } & \% \text { total } \\ \text { NB1-2815-1 } & 20.93 & 68.61 & 25.43 \\ \text { NB1-2815-2 } & 22.85 & 61.33 & 22.13 \\ \text { NB1-2815-3 } & 13.58 & 71.14 & 18.33 \\ \text { NB1-2815-4 } & 21.77 & 70.80 & 28.41 \\ \text { NB1-2815-5 } & 17.52 & 78.21 & 32.20 \\ \text { NB1-2815-6 } & 13.18 & 75.30 & 28.50 \\ \text { NB1-2815-7 } & 15.22 & 70.33 & 24.08 \\ \text { NB1-2815-8 } & 18.01 & 82.72 & 33.47 \\ \text { NB1-2815-9 } & 12.39 & 79.14 & 29.58 \\ \text { NB1-2815-10 } & 11.40 & 82.79 & 30.36 \\ \text { NB1-2815-11 } & 15.34 & 82.40 & 32.50 \\ \text { NB1-2815-12 } & 6.83 & 84.32 & 31.46 \\ \text { NB1-2815-13 } & 8.34 & 82.24 & 30.66 \\ \text { NB1-2815-14 } & 13.37 & 82.56 & 34.48 \\ \text { NB1-2815-15 } & 1.85 & 80.02 & 38.56 \\ \text { NB1-2815-16 } & 1.27 & 83.35 & 45.58 \\ \text { NB1-2815-17 } & 2.51 & 72.22 & 28.11\end{array}$

Flux is in $m g / m^{\wedge} 2 /$ day.

Insufficient material to analyze $91 \mathrm{~mm}$ fraction. 
Carbon Flux at Jan Mayen $(\mathrm{NB}-1)$. 2815m, 1985-86

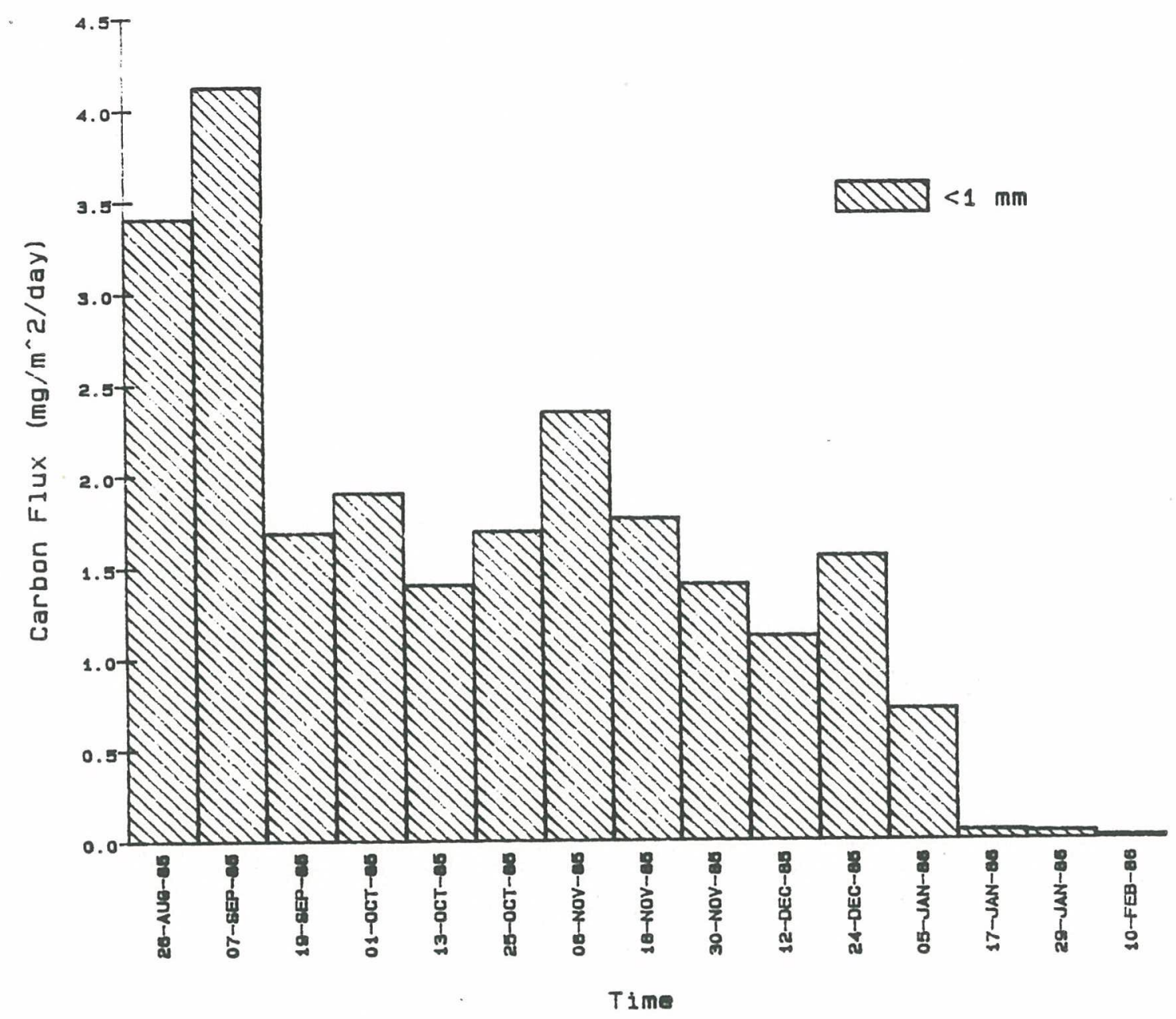

\begin{tabular}{|c|c|c|}
\hline $\begin{array}{l}\text { Sample } \\
\text { I.D. }\end{array}$ & $\begin{array}{l}\text { CRNC } \\
<1\end{array}$ & $\begin{array}{l}\text { CRNC } 1 \\
\% \text { cmb } f .\end{array}$ \\
\hline 70 NB 1-2815-1 & 3.41 & 41.63 \\
\hline 71 NE 1-2815-2 & 4.13 & 36.16 \\
\hline 72 NB1-2815-3 & 1.69 & 23.20 \\
\hline $73 \mathrm{NB} 1-2815-4$ & 1.91 & 24.25 \\
\hline $74 N B 1-2815-5$ & 1.41 & 25.17 \\
\hline 75 NB1-2815-6 & 1.70 & 44.73 \\
\hline $7 E$ NB 1-2815-7 & 2.35 & 13.77 \\
\hline 77 NB $1-2815-8$ & 1.77 & 47.97 \\
\hline 78 NB1-2815-9 & 1.41 & 37.0 \\
\hline $79 N B 1-2815-10$ & 1.12 & 36.9 \\
\hline $80 N B 1-2815-11$ & 1.56 & 39.0 \\
\hline $81 N B 1-2815-12$ & 0.72 & 39.4 \\
\hline $82 N B 1-2815-13$ & 0.05 & 2.2 \\
\hline 83 NB 1-2815-14 & 0.04 & 1.3 \\
\hline $84 N B 1-2815-15$ & 0.02 & 1. \\
\hline
\end{tabular}

Flux is in mg/m $2 /$ day.

"\%mbf" = "\% of combustible flux".

Not enough $1 \mathrm{~mm}$ fraction to do analysis. 
Nitrogen Flux at Jan Mayen (NB-1). 2815m, 1985-86

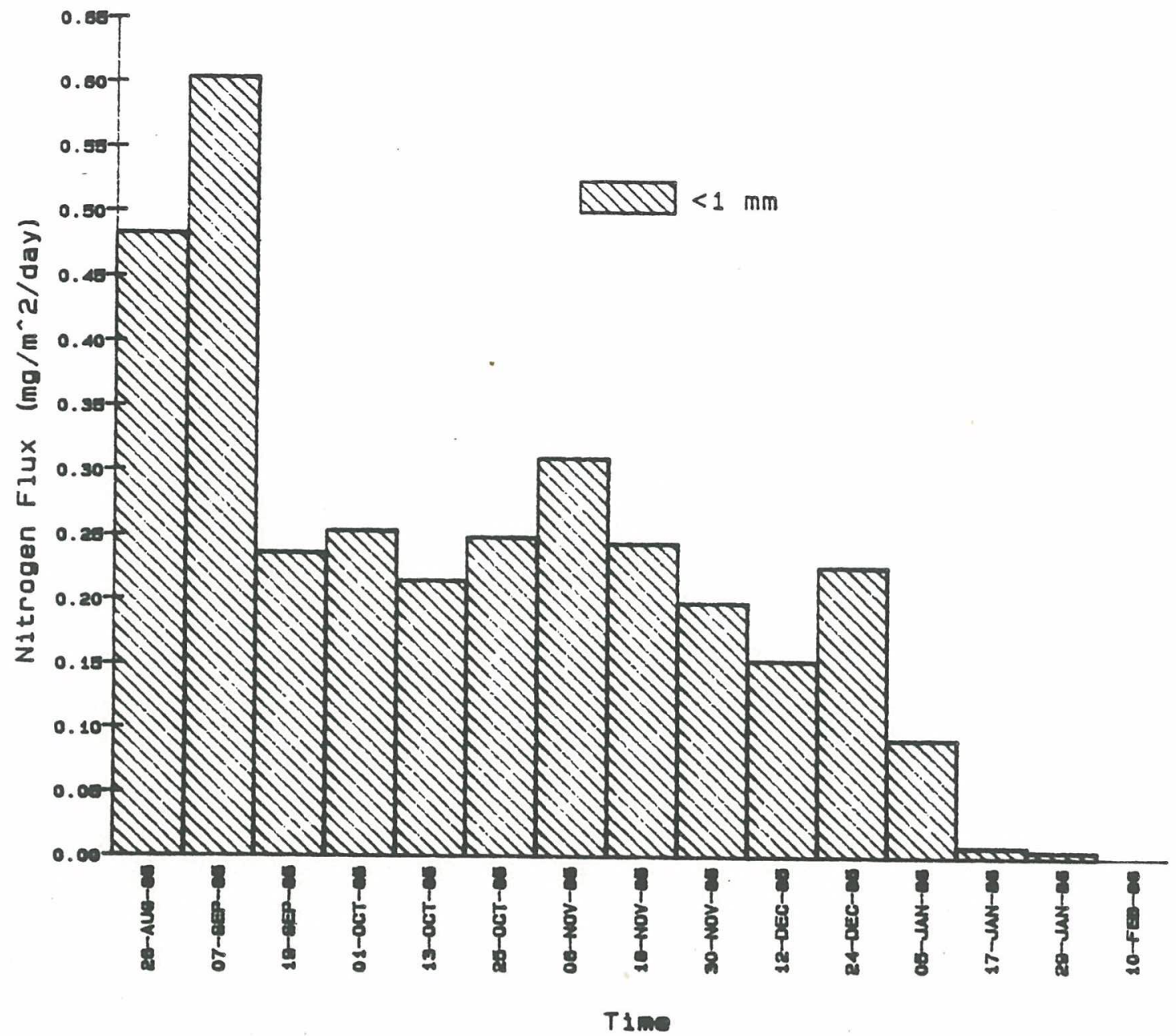

\begin{tabular}{|c|c|c|}
\hline $\begin{array}{l}\text { Gample } \\
\text { I. } . \text {. }\end{array}$ & $\begin{array}{l}\text { NTGN } \\
<1\end{array}$ & $\begin{array}{l}\text { NTGN<1 } \\
\text { \%cmb f. }\end{array}$ \\
\hline 70 NB1-2815-1 & 0.48 & 5.90 \\
\hline 71 NB1-2815-2 & 0.60 & 5.29 \\
\hline 72 NB1-2815-3 & 0.24 & 3.24 \\
\hline 73 NB 1-2815-4 & 0.25 & 3.23 \\
\hline 74 NB1-2815-5 & 0.22 & 3.85 \\
\hline $75 N B 1-2815-6$ & 0.25 & 6.55 \\
\hline 76 NB1-2815-7 & 0.31 & 1.82 \\
\hline 77 NB1-2815-8 & 0.24 & 6.64 \\
\hline 78 NB1-2815-9 & 0.20 & 5.21 \\
\hline 79 NB $1-2815-10$ & 0.15 & 5.04 \\
\hline $80 N B 1-2815-11$ & 0.23 & 5.67 \\
\hline 81 NB $1-2815-12$ & 0.09 & 5.08 \\
\hline $82 \mathrm{NB} 1-2815-13$ & 0.01 & 0.36 \\
\hline 83 NB $1-2815-14$ & 0.01 & 0.17 \\
\hline $84 N B 1-2815-15$ & 0.00 & 0.00 \\
\hline
\end{tabular}

Flux is in $m g / m$ "2/day.

"\%cmbf" = "\% of combustible flux".

Not enough $>1 \mathrm{~mm}$ fraction to do analysis. 
Hydrogen Flux at Jan Mayen (NB-1), 2815m, 1985-86

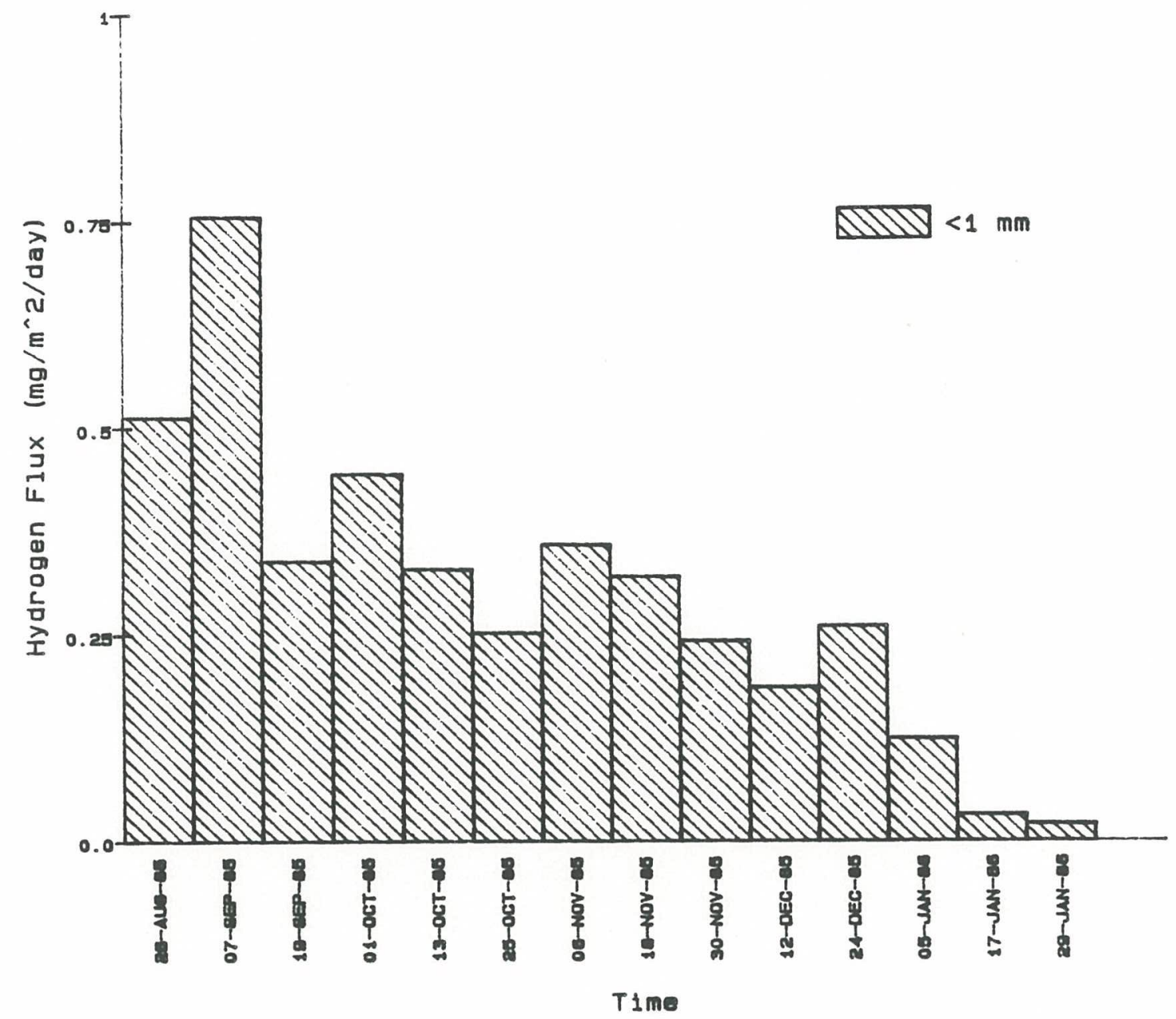

\begin{tabular}{lrr} 
Sample & HYDC & HYDC<1 \\
I.0. & $<1$ & \%cmbf. \\
\hline 70 NB1-2815-1 & 0.51 & 6.28 \\
71 NE1-2815-2 & 0.76 & 6.63 \\
72 NB1-2815-3 & 0.34 & 4.67 \\
73 NB1-2815-4 & 0.45 & 5.69 \\
74 NB1-2815-5 & 0.33 & 5.92 \\
75 NB1-2815-6 & 0.25 & 6.66 \\
76 NB1-2815-7 & 0.36 & 2.11 \\
77 NB1-2815-8 & 0.32 & 8.74 \\
78 NB1-2815-9 & 0.24 & 6.42 \\
79 NB1-2815-10 & 0.19 & 6.14 \\
80 NB1-2815-11 & 0.26 & 6.55 \\
81 NB1-2815-12 & 0.13 & 6.88 \\
82 NB1-2815-13 & 0.03 & 1.35 \\
83 NB1-2815-14 & 0.02 & 0.63
\end{tabular}

Flux is in $m g / m^{*} 2 / d a y$.

"\%ombf" = "\% of combustible flux".

Not enough >1 mm fraction to do analysis. 


\section{EAST GREENLAND/FRAM STRATT AREA}




\section{FS-1 \\ CENTRAL RRAM STRAIT \\ $78^{\circ} 52^{\prime} \mathrm{N}, 01^{\circ} 22^{\prime} \mathrm{E}$}

Trap depth: 2,440m Water depth: 2,527m

Annual Fluxes $\left(\mathrm{g} / \mathrm{m}^{2} / \mathrm{yr}\right)$ :

Total..................6.6.61

Carbonate................1.40

Noncombustible ........4.26

Combustible............0.92

Lithogenic ............4.00

Biogenic Opal .........0.60

Organic C................41

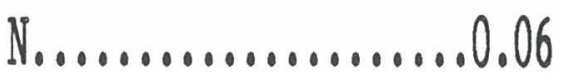

PAPFLUK Mark 5-13

\begin{tabular}{|c|c|c|c|c|}
\hline $\begin{array}{l}\text { Sample } \\
\text { ID }\end{array}$ & $\begin{array}{l}\text { Opening } \\
\text { Date }\end{array}$ & $\begin{array}{l}\text { Closing } \\
\text { Date }\end{array}$ & Span & $\begin{array}{l}\text { Mid. } \\
\text { Date }\end{array}$ \\
\hline & & & & \\
\hline $13 F S 1-2000-1$ & $20-A \cup G-84$ & $17-S E P-84$ & 27.5 & $03-5 E P-84$ \\
\hline 14 FS1-2000-2 & $17-5 E P-84$ & $.15-0 \mathrm{CT}-84$ & 27.5 & $01-0 \mathrm{CT}-84$ \\
\hline $15 \mathrm{FS} 1-2000-3$ & $15-0 C T-84$ & $11-$ NOV -84 & 27.5 & $29-0 C T-84$ \\
\hline 16 FS 1-2000-4 & $11-$ NOV -84 & $99-D E C-84$ & 27.5 & $25-\mathrm{NOV}-84$ \\
\hline 17 F51-2000-5 & $99-D E C-84$ & $97-J A N-85$ & 27.5 & 24-DEC-84 \\
\hline $18 F S 1-2000-6$ & $97-J A N-85$ & $03-F E B-85$ & 27.5 & $21-J A N-85$ \\
\hline $19 F S 1-2000-7$ & $03-F E B-85$ & DS-MAR-85 & 27.5 & $17-F E B-85$ \\
\hline 20 FS 1-2000-8 & DJ-MAR-85 & $30-M A R-85$ & 27.5 & 17-MAR-85 \\
\hline 21 FS 1-2000-9 & $30-M A R-85$ & $27-A P R-85$ & 27.5 & $13-A P R-85$ \\
\hline 22 FS1-2000-10 & $27-A P R-85$ & $25-M A Y-85$ & 27.5 & $11-M A Y-85$ \\
\hline 23 FS1-2000-11 & $25-M A Y-85$ & $21-J U N-85$ & 27.5 & $08-J U N-85$ \\
\hline 24 FS1-2000-12 & $21-J \cup N-85$ & 19-JUL-85 & 27.5 & $05-J U L-85$ \\
\hline 25 FS1-2000-13 & $19-J U L-85$ & $15-A \cup G-85$ & 27.5 & $02-A \cup G-85$ \\
\hline
\end{tabular}


Total Flux at Fram Strait (FS-1). 2000m. 1984-1985

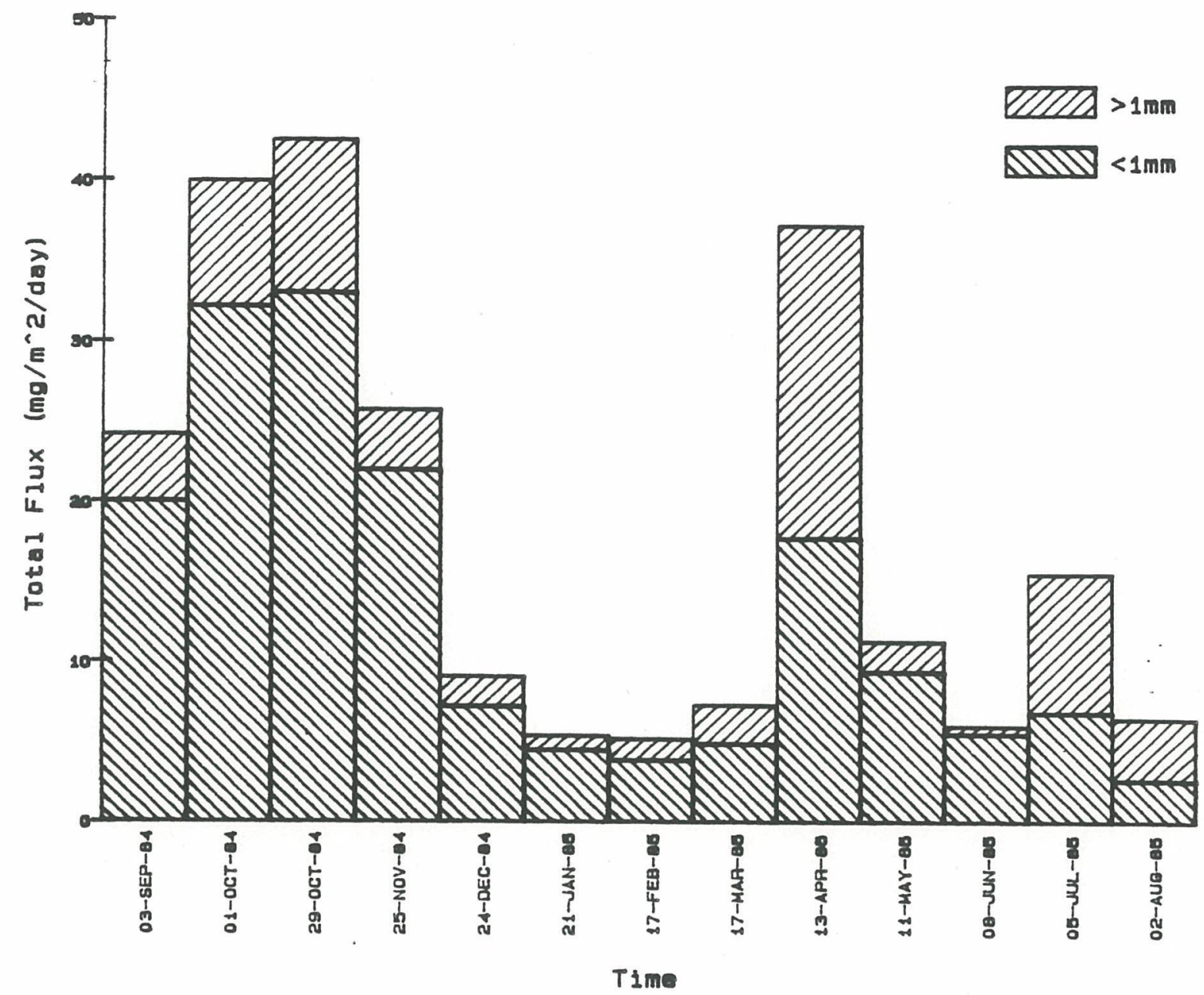

FRAM STRAIT 1 POISONED WITH HB CL2 359 DAYS

Mark 5 trap open from AUGUST 201984 to AUGUST 151985 at 2000 meters. TOTAL FLUX (mg $/ \mathrm{n}^{\wedge} 2$, day)

Ttl is total Flux in all size classes

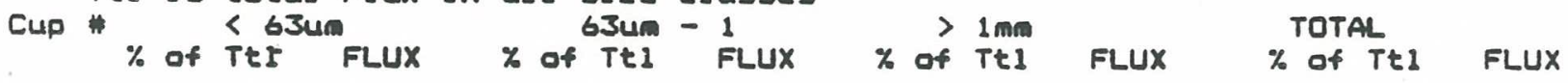

\begin{tabular}{|c|c|c|c|c|c|c|c|c|}
\hline 1 & $\begin{array}{l}57.18 \\
63.02\end{array}$ & $\begin{array}{l}12.30 \\
25.34\end{array}$ & $\begin{array}{l}23.52 \\
17.56\end{array}$ & $\begin{array}{l}5.06 \\
7.06\end{array}$ & $\begin{array}{l}19.29 \\
19.42\end{array}$ & $\begin{array}{l}4.15 \\
7.81\end{array}$ & $\begin{array}{l}100.00 \\
100.00\end{array}$ & $\begin{array}{l}21.51 \\
40.21\end{array}$ \\
\hline 3 & 64.12 & 29.17 & 14.99 & 6.82 & 20.89 & 9.50 & 100.00 & 45.49 \\
\hline 4 & 50.03 & 9.05 & 29.41 & 5.32 & 20.56 & 3.72 & 100.00 & 18.09 \\
\hline 5 & 63.83 & 6.46 & 17.89 & 1.81 & 18.28 & 1.85 & 100.00 & 10.12 \\
\hline 6 & 52.92 & 3.90 & 34.60 & 2.55 & 12.48 & .92 & 100.00 & 7.37 \\
\hline 7 & 59.75 & 2.91 & 12.73 & .62 & 27.52 & 1.34 & 100.00 & 4.87 \\
\hline 8 & 51.98 & 3.80 & 15.18 & 1.11 & 32.83 & 2. 40 & 100.00 & 7.31 \\
\hline 9 & 24.06 & 14.00 & 27.73 & 15.62 & 47.41 & 26.70 & 100.00 & 56.32 \\
\hline 10 & 36.46 & 3.81 & 45.45 & 4.75 & 18.09 & 1.89 & 100.00 & 10.45 \\
\hline 11 & 72.32 & 4.39 & 19.60 & 1.19 & 8.07 & .49 & 100.00 & 6.07 \\
\hline 12 & 31.80 & 5.31 & 16.11 & 2.69 & 52.10 & 8.70 & 100.00 & $16.7 C$ \\
\hline 13 & 31.56 & 2.13 & 12.00 & .81 & 56.44 & 3.81 & 100.00 & 6.75 \\
\hline
\end{tabular}


Carbonate Flux at Fram Strait (FS-1), 2000m, 1984-1985

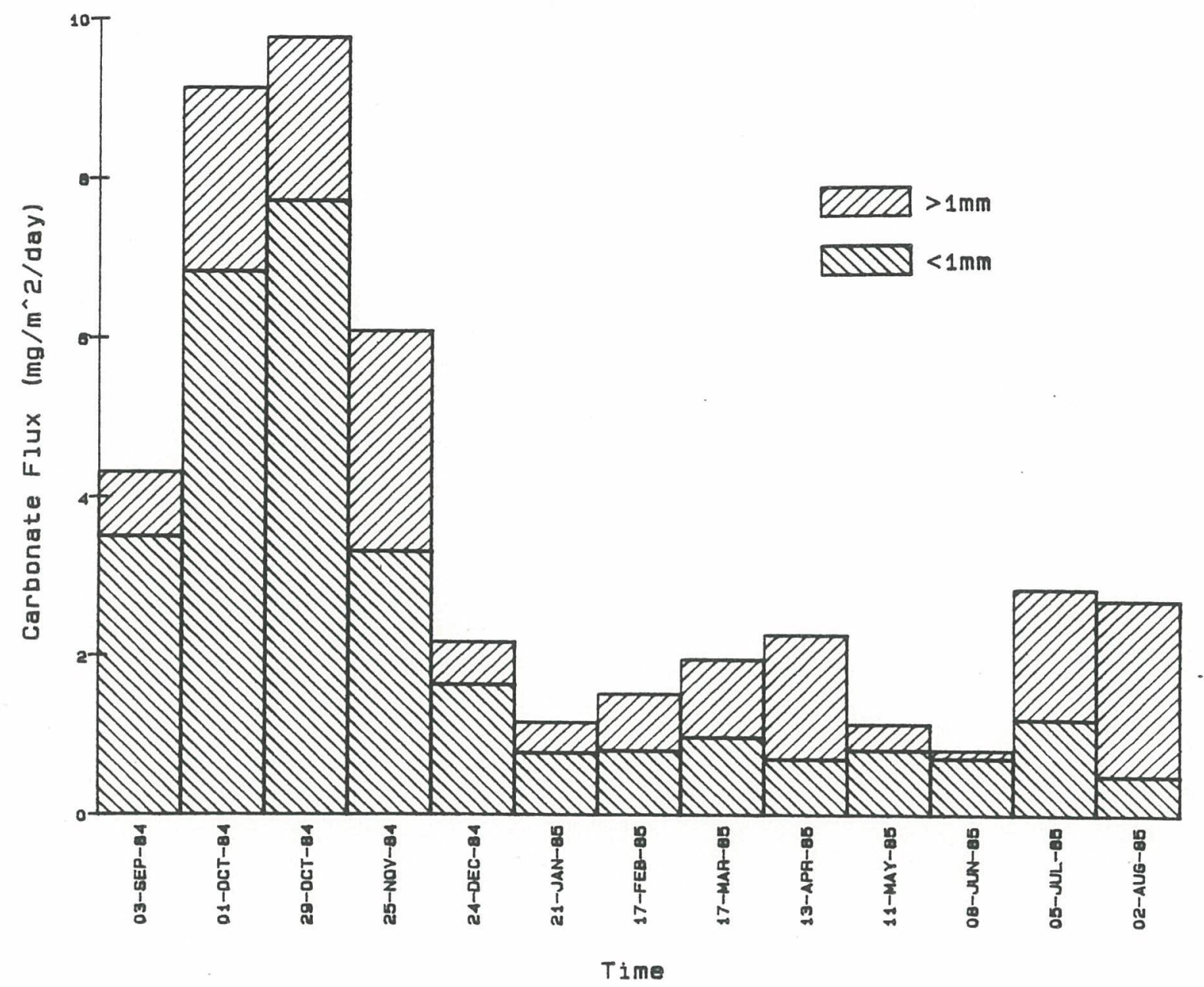

\begin{tabular}{|c|c|c|c|c|c|c|}
\hline $\begin{array}{l}\text { Sample } \\
\text { I.D. }\end{array}$ & $\begin{array}{l}\text { CRTA } \\
<1\end{array}$ & $\begin{array}{l}\text { CRTA } \% \\
\text { tot. }<1\end{array}$ & $\begin{array}{l}\text { CRTA } \\
>1\end{array}$ & $\begin{array}{l}\text { CRTA \% } \\
\text { tot. }>1\end{array}$ & $\begin{array}{l}\text { CRTA } \\
\text { total }\end{array}$ & $\begin{array}{l}\text { CRTA } \% \\
\text { total }\end{array}$ \\
\hline 13 FS1-2000-1 & 3.51 & 14.52 & 0.81 & 3.77 & 4.32 & 3.35 \\
\hline 14 FS1-2000-2 & 6.84 & 17.13 & 2.30 & 5.72 & 9.14 & 5.76 \\
\hline $15 \mathrm{FS} 1-2000-3$ & 7.72 & 18.16 & 2.05 & 4.51 & 9.77 & 4.82 \\
\hline 16 FS1-2000-4 & 3.31 & 18.33 & 2.78 & 15.37 & 6.09 & 15.39 \\
\hline $17 \mathrm{FS} 1-2000.5$ & 1.54 & 18.10 & 0.54 & 5.34 & 2.18 & 5.96 \\
\hline 18 F51-2000-6 & 0.78 & 14.44 & 0.39 & 3.85 & 1.17 & 7.22 \\
\hline 19 FS1-2000-7 & 0.81 & 15.52 & 0.72 & 9.78 & 1.53 & 13.79 \\
\hline $20 F S 1-2000-8$ & 0.98 & 13.44 & 0.98 & 13.41 & 1.96 & 13.44 \\
\hline $21 F 51-2000-9$ & 0.71 & 1.60 & 1.57 & 2.79 & 2.28 & 3.53 \\
\hline 22 FS $1-2000-10$ & 0.82 & 7.27 & 0.33 & 3.16 & 1.15 & 2.93 \\
\hline 23 FS1-2000-11 & 0.72 & 11.98 & 0.10 & 1.65 & 0.82 & 1.66 \\
\hline 24 FS $1-2000-12$ & 1.21 & 7.79 & 1.64 & 9.80 & 2.85 & 10.56 \\
\hline 25 F $51-2000-13$ & 0.50 & 7.76 & 2.21 & 32.74 & 2.71 & 34.32 \\
\hline
\end{tabular}

Flux is in mg/m"2/day. 
Noncombustible Flux at Fram Strait 1. 2000 m. 1984-85

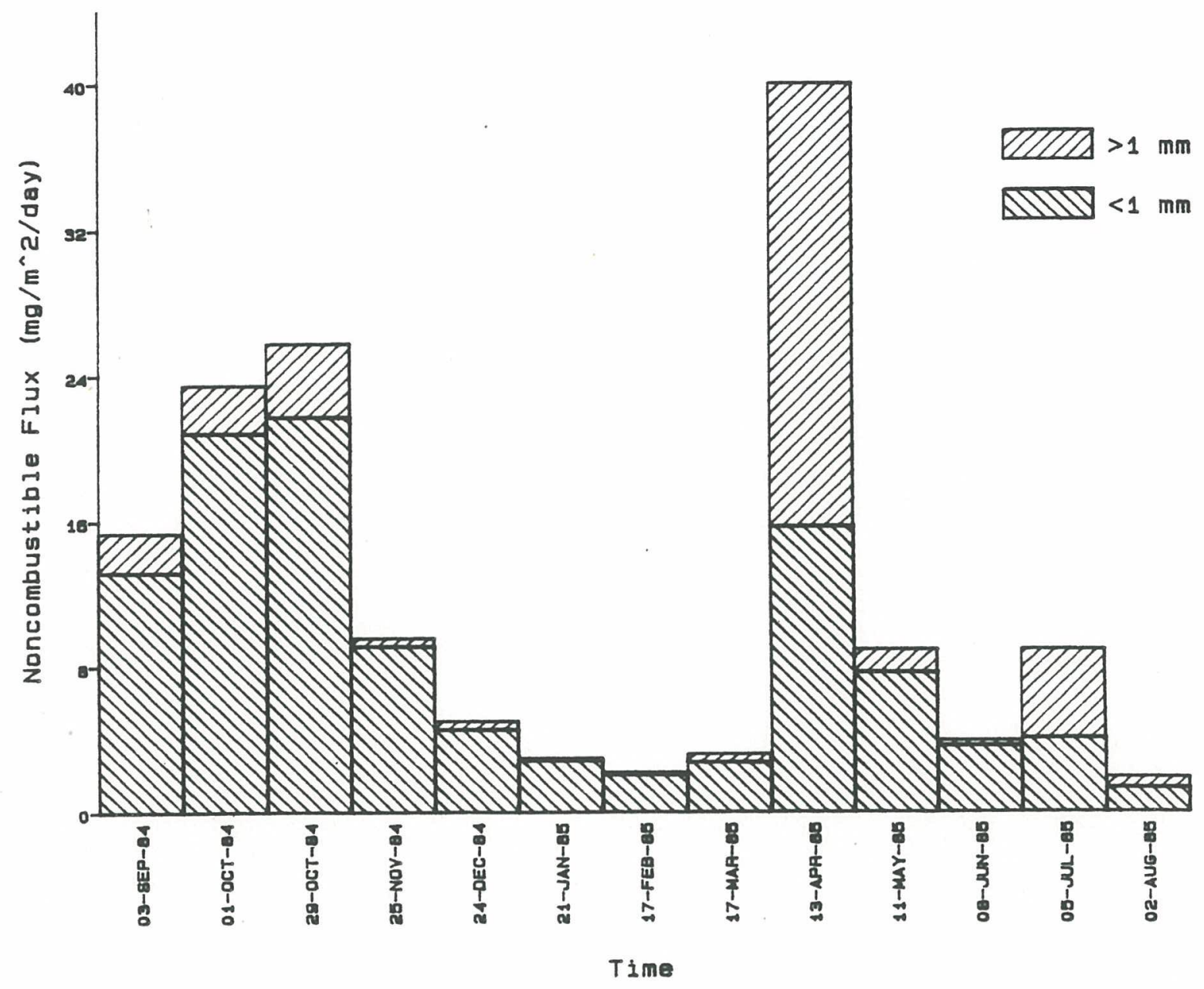

\begin{tabular}{|c|c|c|c|c|c|c|}
\hline $\begin{array}{l}\text { Sample } \\
\text { ID\# }\end{array}$ & $\begin{array}{l}\text { NONC } \\
<1\end{array}$ & $\begin{array}{l}\text { NONC } \% \\
\text { tot. }<1\end{array}$ & $\begin{array}{l}\text { NONC } \\
>1\end{array}$ & $\begin{array}{l}\text { NONC } \% \\
\text { tot. }>1\end{array}$ & $\begin{array}{l}\text { NONC } \\
\text { total }\end{array}$ & $\begin{array}{l}\text { NONC } \% \\
\text { total }\end{array}$ \\
\hline & & & & & & - - - - \\
\hline 13 F51-2000-1* & 13.22 & 54.67 & 2.14 & 8.85 & 15.36 & 63.52 \\
\hline $14 F 51-2000-2 *$ & 20.86 & 52.23 & 2.64 & 6.61 & 23.50 & 58.84 \\
\hline 15 FS1-2000-3* & 21.77 & 51.21 & 4.02 & 9.46 & 25.79 & 50.67 \\
\hline $16 F 51-2000-4 *$ & 9.16 & 50.72 & 0.45 & 2.49 & 9.61 & 53.21 \\
\hline 17 FS $1-2000-5 *$ & 4.56 & 50.33 & 0.45 & 4.97 & 5.01 & 55.30 \\
\hline 18 FS 1-2000-6* & 2.87 & 53.15 & 0.13 & 2.41 & 3.00 & 55.56 \\
\hline $19 F S 1-2000-7 *$ & 2.06 & 39.46 & 0.13 & 2.49 & 2.29 & 43.87 \\
\hline 20 FS1-2000-8* & 2.75 & 37.72 & 0.47 & 6.45 & 3.22 & 44.17 \\
\hline 21 FS1-2000-9* & 15.74 & 35.43 & 24.30 & 54,69 & 40.04 & 90.12 \\
\hline 22 FS1-2000-10* & 7.67 & 58.00 & 1.23 & .10 .90 & 8.90 & 78.90 \\
\hline 23 FS1-2000-11* & 3.67 & 61.06 & 0.29 & 4.83 & 3.96 & 65.89 \\
\hline $24 F 51-2000-12 *$ & 4.09 & 26.34 & 4.92 & 31.68 & 9.01 & 58.02 \\
\hline 25 FS1-2000-13* & 1.35 & 20.96 & 0.59 & 9.16 & 1.94 & 30.12 \\
\hline
\end{tabular}

Flux is in $m g / m^{\wedge} 2 / d a y$. 
Combustible Flux at Fram Strait 1. 2000 m. 1984-85

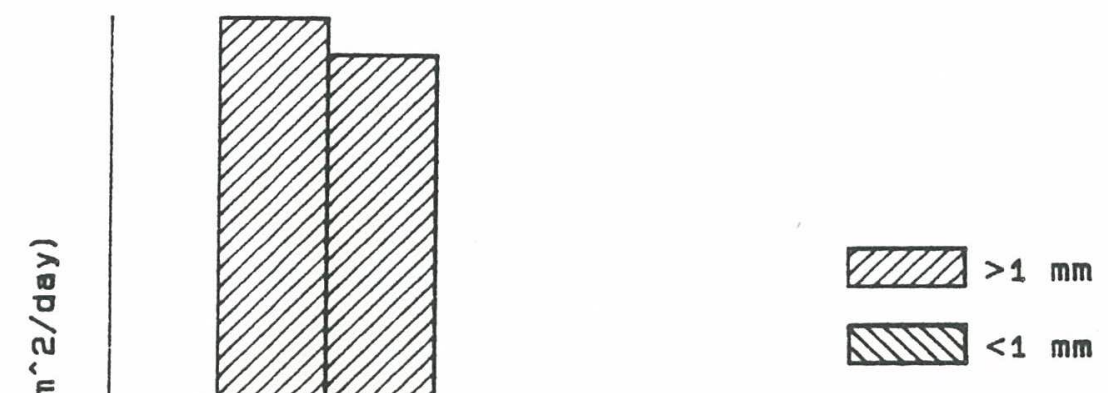

\begin{tabular}{|c|c|c|c|c|c|c|}
\hline $\begin{array}{l}\text { Sample } \\
\text { ID\# }\end{array}$ & $\begin{array}{l}\text { COMB } \\
<1\end{array}$ & $\begin{array}{l}\text { COMB } \% \\
\text { tot.<1 }\end{array}$ & $\begin{array}{l}\text { COMB } \\
y 1\end{array}$ & $\begin{array}{l}\text { COMB } \% \\
\text { tot. }>1\end{array}$ & $\begin{array}{l}\text { COMB } \\
\text { TOTAL }\end{array}$ & $\begin{array}{l}\text { CoMB } \% \\
\text { total }\end{array}$ \\
\hline 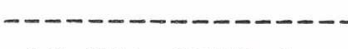 & & & & & & --- \\
\hline 13 FS 1-2000-1* & 3.28 & 13.56 & 1.21 & 5.00 & 4.49 & 18.57 \\
\hline $14 F 51-2000-2 *$ & 4.32 & 10.82 & 2.87 & 7.19 & 7.19 & 18.00 \\
\hline 15 F $51-2000-3 *$ & 3.51 & 8.26 & 3.43 & 8.07 & 6.94 & 16.33 \\
\hline 16 FS $1-2000-4 *$ & 1.61 & 8.91 & 0.49 & 2.71 & 2.10 & 11.63 \\
\hline 17 FS $1-2000-5 *$ & 0.85 & 9.38 & 0.86 & 9.49 & 1.71 & 18.87 \\
\hline 18 FS $1-2000-6 *$ & 0.43 & 7.96 & 0.39 & 7.22 & 0.82 & 15.19 \\
\hline 19 FS $1-2000-7 *$ & 0.31 & 5.94 & 0.49 & 9.39 & 0.80 & 15.33 \\
\hline 20 FS $1-2000-8 *$ & 0.45 & 6.17 & 0.95 & 13.03 & 1.40 & 19.20 \\
\hline $21 F 51-2000-9 *$ & 0.58 & 1.31 & 0.83 & 1.87 & 1.41 & 3.17 \\
\hline 22 FS $1-2000-10 *$ & 0.55 & 4.88 & 0.33 & 2.93 & 0.88 & 7.80 \\
\hline 23 FS 1-2000-11* & 0.68 & 11.31 & 0.10 & 1.66 & 0.78 & 12.98 \\
\hline 24 FS $1-2000-12 *$ & 0.85 & 5.47 & 2.13 & 13.72 & 2.98 & 19.19 \\
\hline 25 FS1-2000-13* & 0.27 & 4.19 & 1.01 & 15.68 & 1.28 & 19.88 \\
\hline
\end{tabular}

Flux is in $m g / m^{\prime \prime} 2 / d a y$. 
Blogenic Opal Flux at Fram Strait 1. 2000m. 1984-85

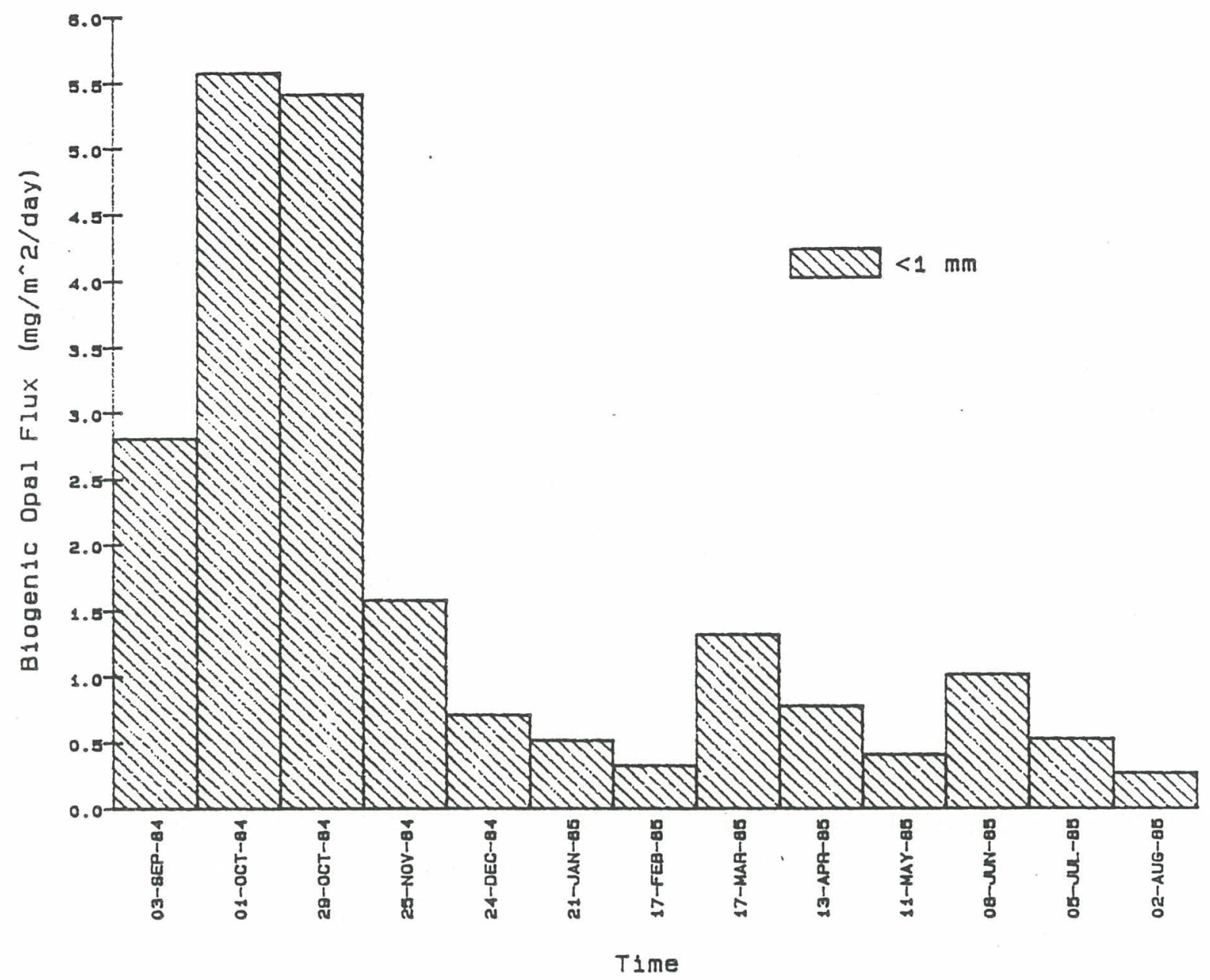

\begin{tabular}{lrrr} 
Sample & OPAL & \multicolumn{1}{c}{ OPAL<1 } & \multicolumn{1}{c}{ OPAL<1 } \\
ID\# & 1 & \%NCMb. & $\%$ Total \\
\hline 13 FS1-2000-1 & 2.81 & 18.28 & 11.61 \\
14 FS1-2000-2 & 5.58 & 23.73 & 13.96 \\
15 FS1-2000-3 & 5.41 & 20.98 & 12.73 \\
16 FS1-2000-4 & 1.58 & 16.46 & 8.76 \\
17 FS1-2000-5 & 0.71 & 14.23 & 7.87 \\
18 FS1-2000-6 & 0.52 & 17.33 & 9.63 \\
19 FS1-2000-7 & 0.33 & 14.47 & 6.35 \\
20 FS1-2000-8 & 1.32 & 40.91 & 18.07 \\
21 FS1-2000-9 & 0.78 & 1.95 & 1.75 \\
22 FS1-2000-10 & 0.41 & 4.63 & 3.65 \\
23 FS1-2000-11 & 1.02 & 25.73 & 16.96 \\
24 FS1-2000-12 & 0.53 & 5.93 & 3.44 \\
25 FS1-2000-13 & 0.27 & 14.14 & 4.26
\end{tabular}

Flux is in $m g / m^{\prime \prime} 2 /$ day.

\%Ncmb. is "\% noncombustible flux".

Not enough $>1 \mathrm{~mm}$ fraction to do analysis. 


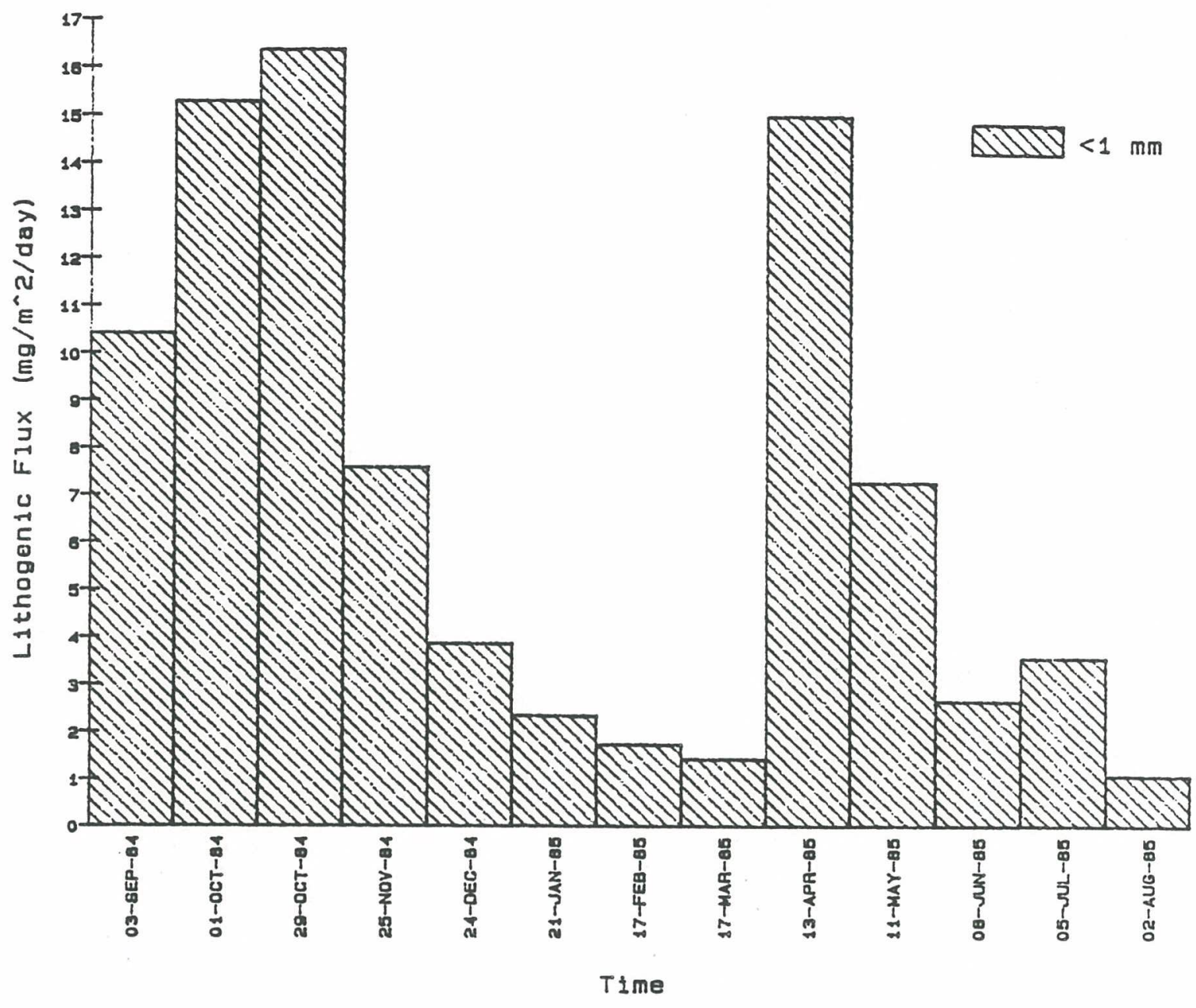

\begin{tabular}{lrr} 
Gample & LITH & LITH \\
ID\# & $\langle 1$ & \%Ncmb. \\
\hline 13 FS1-2000-1 & 10.41 & 67.79 \\
14 FS1-2000-2 & 15.28 & 65.03 \\
15 FS1-2000-3 & 16.36 & 63.44 \\
16 FS1-2000-4 & 7.58 & 78.86 \\
17 FS $1-2000-5$ & 3.85 & 76.79 \\
18 FS1-2000-6 & 2.35 & 78.34 \\
19 FS1-2000-7 & 1.73 & 75.49 \\
20 FS1-2000-8 & 1.43 & 44.49 \\
21 FS1-2000-9 & 14.96 & 37.36 \\
22 FS1-2000-10 & 7.26 & 81.55 \\
23 FS1-2000-11 & 2.65 & 66.94 \\
24 FS1-2000-12 & 3.56 & 39.46 \\
25 FS1-2000-13 & 1.08 & 55.45
\end{tabular}

Flux is in $m g / m^{\wedge} 2 /$ day.

\% Ncmb. is "\% noncombustible flux".

Not enough $>1 \mathrm{~mm}$ fraction to do analysis. 
Carbon Flux at Fram Strait 1. 2000m, 1984-85

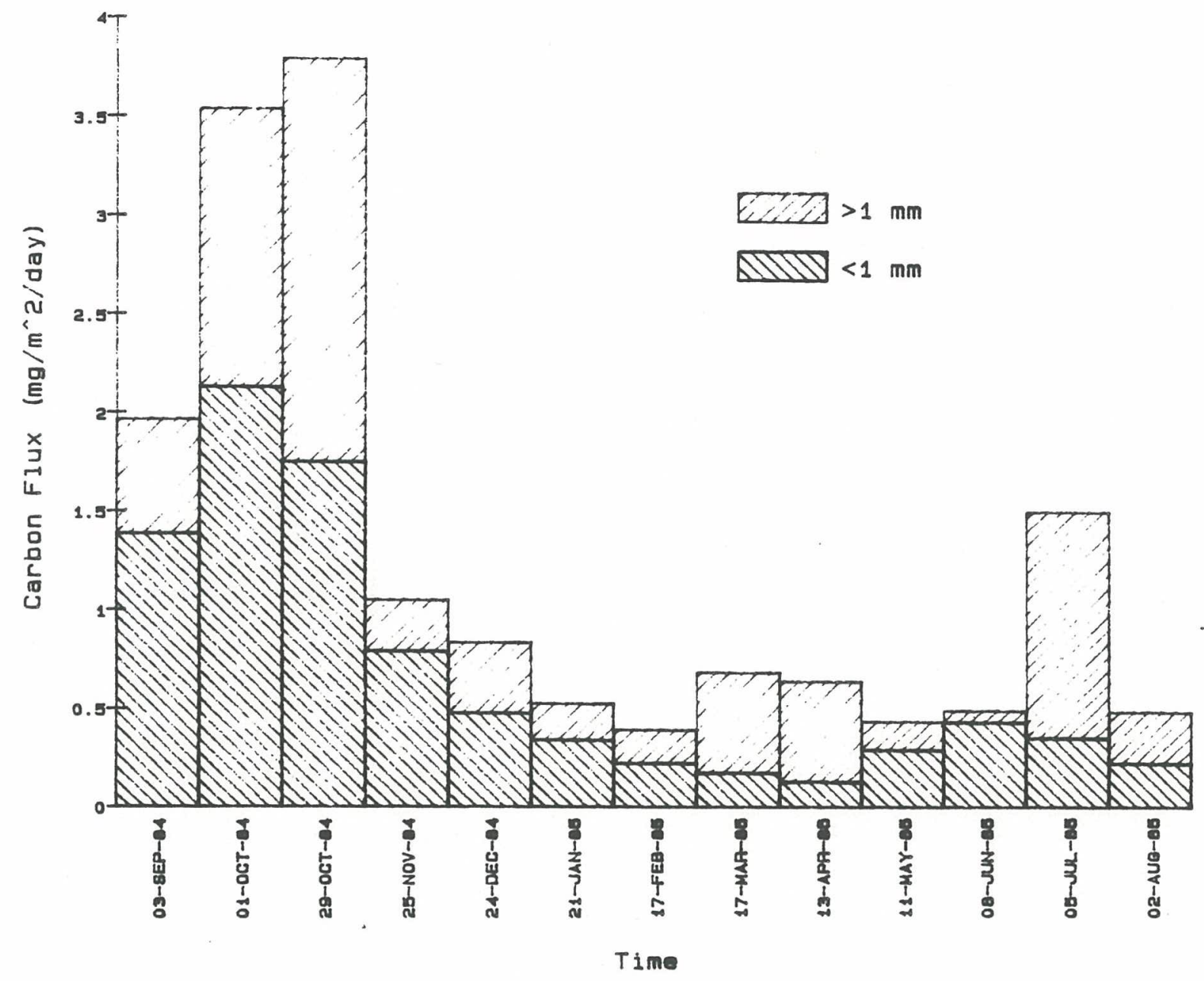

\begin{tabular}{|c|c|c|c|c|c|c|}
\hline $\begin{array}{l}\text { Sample } \\
\text { I.D. }\end{array}$ & $\begin{array}{l}\text { CRNC } \\
<1\end{array}$ & $\begin{array}{l}\text { CRNC }<1 \\
\% \text { cmb } f .\end{array}$ & $\begin{array}{l}\text { CRNC } \\
>1\end{array}$ & $\begin{array}{l}\text { CRNC } 1 \\
\% \text { cmb } f .\end{array}$ & $\begin{array}{l}\text { CRNC } \\
\text { total }\end{array}$ & $\begin{array}{l}\text { CRNCtot. } \\
\text { \%cmb } f .\end{array}$ \\
\hline 13 FS $1-2000-1 *$ & 1.39 & 30.89 & 0.58 & 12.92 & 1.97 & 43.81 \\
\hline 14 FS1-2000-2* & 2.13 & 29.62 & 1.41 & 19.61 & 3.54 & 49.23 \\
\hline 15 FS1-2000-3* & 1.75 & 25.25 & 2.04 & 29.39 & 3.79 & 54.65 \\
\hline 16 FS $1-2000-4 *$ & 0.79 & 37.66 & 0.26 & 12.38 & 1.05 & 50.04 \\
\hline 17 FS $1-2000-5 *$ & 0.48 & 27.80 & 0.36 & 21.05 & 0.84 & 48.85 \\
\hline 18 FS1-2000-6* & 0.34 & 41.45 & 0.19 & 22.71 & 0.53 & 64.16 \\
\hline 19 FS1-2000-7* & 0.22 & 27.77 & 0.17 & 21.25 & 0.39 & 49.02 \\
\hline $20 F 51-2000-8 *$ & 0.18 & 12.86 & 0.51 & 36.48 & 0.69 & 49.29 \\
\hline 21 FS $1-2000-9 *$ & 0.13 & 9.08 & 0.51 & 36.17 & 0.64 & 45.25 \\
\hline 22 FS $1-2000-10 *$ & 0.29 & 32.92 & 0.14 & 16.22 & 0.43 & 49.14 \\
\hline 23 FS $1-2000-11 *$ & 0.43 & 55.10 & 0.06 & 7.69 & 0.49 & 62.79 \\
\hline 24 FS $1-2000-12 *$ & 0.35 & 11.81 & 1.15 & 38.59 & 1.50 & 50.40 \\
\hline 25 FS $1-2000-13 *$ & 0.22 & 17.39 & 0.26 & 20.31 & 0.48 & 37.70 \\
\hline
\end{tabular}

Flux is in $\mathrm{mg} / \mathrm{m}^{\wedge} 2 /$ day.

" cmbf" = "\% of combustible flux". 


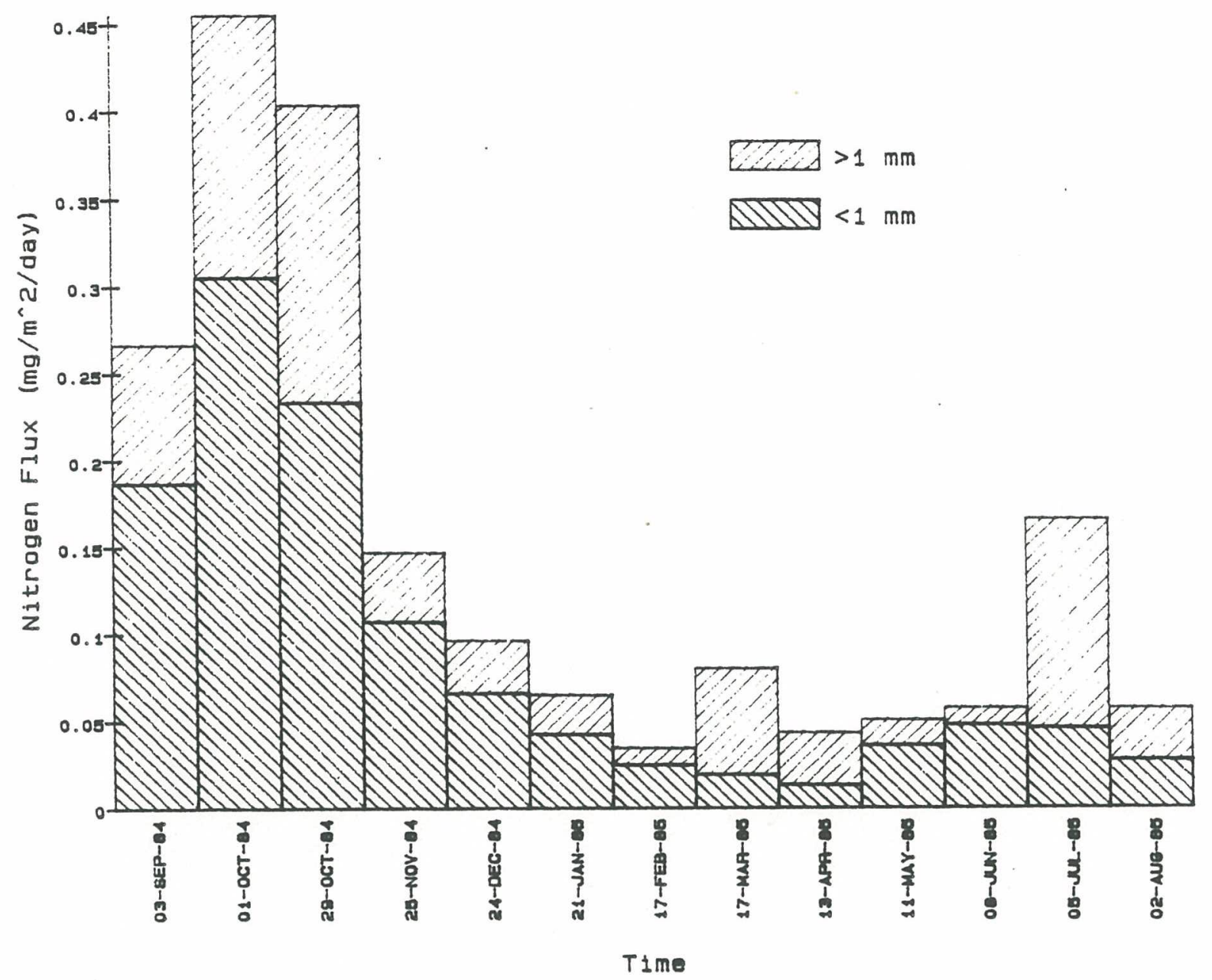

\begin{tabular}{|c|c|c|c|c|c|c|}
\hline $\begin{array}{l}\text { Sample } \\
\text { I.D. }\end{array}$ & $\begin{array}{l}\text { NTGN } \\
<1\end{array}$ & $\begin{array}{l}\text { NTGN<1 } \\
\% \text { cmb f. }\end{array}$ & $\begin{array}{l}\text { NTGN } \\
>1\end{array}$ & $\begin{array}{l}\text { NTGN>1 } \\
\% \text { cmb } f .\end{array}$ & $\begin{array}{l}\text { NTGN } \\
\text { total }\end{array}$ & $\begin{array}{l}\text { NTGNtot. } \\
\% \text { cmbf. }\end{array}$ \\
\hline $13 F \subseteq 1-2000-1 *$ & 0.19 & 4.17 & 0.08 & 1.78 & 0.27 & 5.95 \\
\hline 14 FS1-2000-2* & 0.30 & 4.24 & 0.15 & 2.09 & 0.45 & 6.33 \\
\hline 15 FS।-2000-3* & 0.23 & 3.36 & 0.17 & 2.45 & 0.40 & 5.81 \\
\hline 16 F51-2000-4* & 0.11 & 5.09 & 0.04 & 1.90 & 0.15 & 6.99 \\
\hline 17 FS $1-2000-5 *$ & 0.07 & 3.86 & 0.03 & 1.75 & 0.10 & 5.61 \\
\hline 18 FS1-2000-6* & 0.04 & 5.21 & 0.02 & 2.77 & 0.07 & 7.98 \\
\hline $19 F \subseteq 1-2000-7 *$ & 0.02 & 3.11 & 0.01 & 1.25 & 0.03 & 4.36 \\
\hline 20 FS $1-2000-8 *$ & 0.14 & 9.87 & 0.06 & 4.35 & 0.20 & 14.22 \\
\hline 21 FS1-2000-9* & 0.01 & 0.95 & 0.03 & 2.13 & 0.04 & 3.08 \\
\hline 22 FS $1-2000-10 *$ & 0.04 & 4.09 & 0.01 & 1.65 & 0.05 & 5.74 \\
\hline 23 FS $1-2000-11 *$ & 0.05 & 6.13 & 0.01 & 1.28 & 0.06 & 7.41 \\
\hline 24 FS1-2000-12* & 0.05 & 1.54 & 0.12 & 4.03 & 0.17 & 5.57 \\
\hline 25 FS $1-2000-13^{*}$ & 0.03 & 2.14 & 0.03 & 2.34 & 0.06 & 4.48 \\
\hline
\end{tabular}

Flux is in $m g / m / 2 / d a y$.

" $\%$ cmbf $"=" \%$ of combustible flux" 


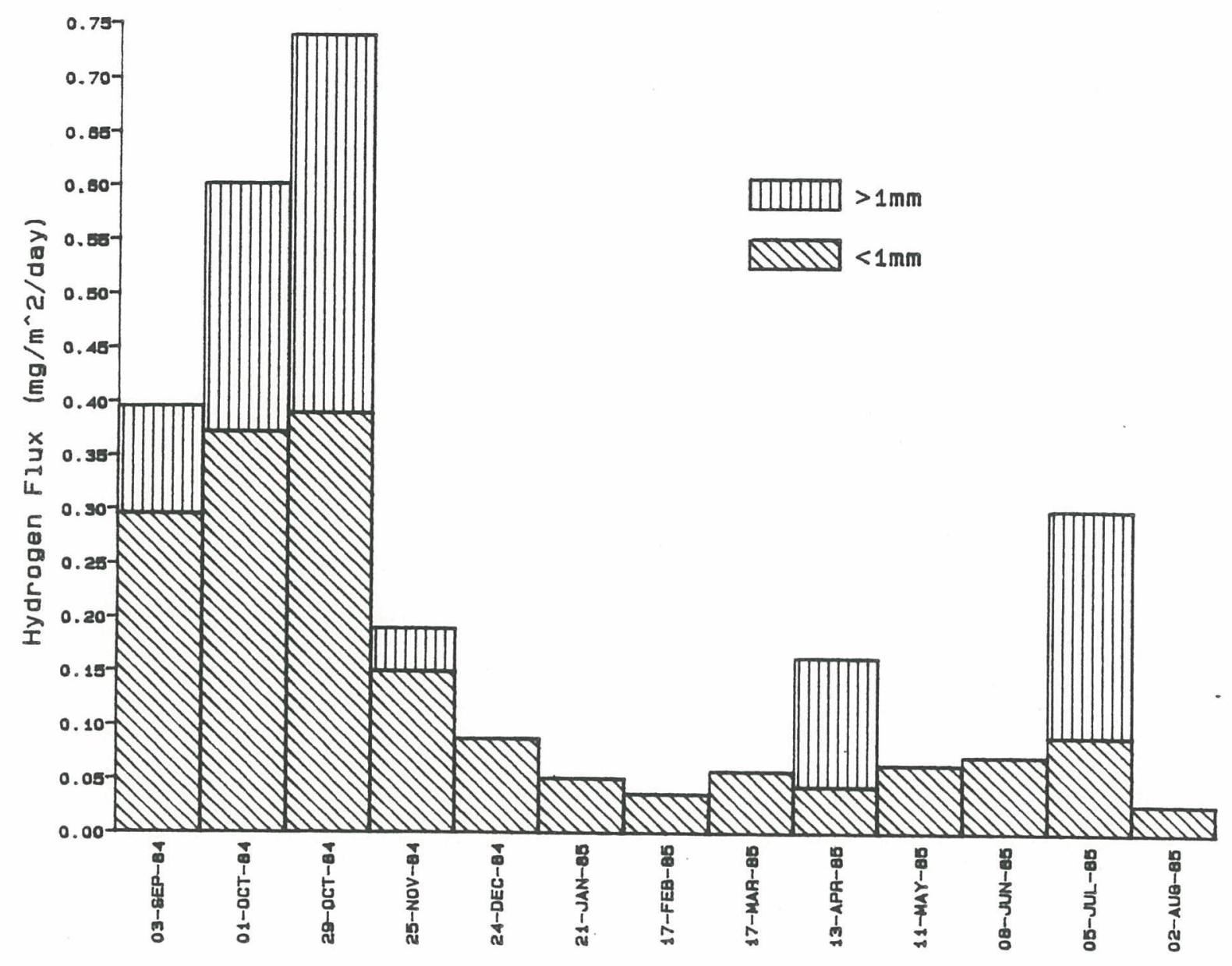

Time

\begin{tabular}{|c|c|c|c|c|c|c|}
\hline $\begin{array}{l}\text { Gample } \\
\text { I.D. }\end{array}$ & $\begin{array}{l}\text { HYDC } \\
<1\end{array}$ & $\begin{array}{l}\text { HYDC } 1 \\
\% \text { cmbf. }\end{array}$ & $\begin{array}{l}\text { HYDC } \\
>1\end{array}$ & $\begin{array}{l}\text { HYDC } 1 \\
\% \text { cmb f. }\end{array}$ & $\begin{array}{l}\text { HYOC } \\
\text { total }\end{array}$ & $\begin{array}{l}\text { HYOCtot. } \\
\% \text { cmbf. }\end{array}$ \\
\hline $13 F S 1-2000-1 *$ & 0.27 & 6.06 & 0.10 & 2.23 & 0.37 & 8.29 \\
\hline $14 F S 1-2000-2 *$ & 0.40 & 5.52 & 0.23 & 3.20 & 0.63 & 8.72 \\
\hline $15 \mathrm{FS} 1-2000-3 *$ & 0.45 & 6.47 & 0.35 & 5.04 & 0.80 & 11.51 \\
\hline $1 E F S 1-2000-4 *$ & 0.17 & 7.95 & 0.04 & 1.90 & 0.21 & 9.86 \\
\hline 17 FS $1-2000-5 *$ & 0.11 & 6.28 & 0.06 & 3.52 & 0.17 & 9.80 \\
\hline 18 FS $1-2000-6 *$ & 0.08 & 9.64 & 0.03 & 3.23 & 0.11 & 12.87 \\
\hline $19 F S 1-2000-7 *$ & 0.04 & 5.54 & 0.03 & 3.87 & 0.08 & 9.40 \\
\hline $20 F \subseteq 1-2000-8 *$ & 0.05 & 32.14 & 0.08 & 5.75 & 0.13 & 21.13 \\
\hline 21 FS1-2000-9* & 0.05 & 3.52 & 0.12 & 8.51 & 0.17 & 12.03 \\
\hline 22 FS $1-2000-10 *$ & 0.06 & 7.30 & 0.05 & 5.35 & 0.11 & 12.66 \\
\hline 23 FS1-2000-11* & 0.08 & 10.76 & 0.01 & 1.51 & 0.10 & 12.27 \\
\hline 24 FS1-2000-12* & 0.07 & 2.39 & 0.21 & 7.05 & 0.28 & 9.43 \\
\hline 25 FS1-2000-13* & 0.04 & 3.01 & 0.05 & 3.77 & 0.09 & 6.75 \\
\hline
\end{tabular}

Flux is in $m g / m$ '2/day.

" $\%$ cmbf" = "\% of combustible flux". 


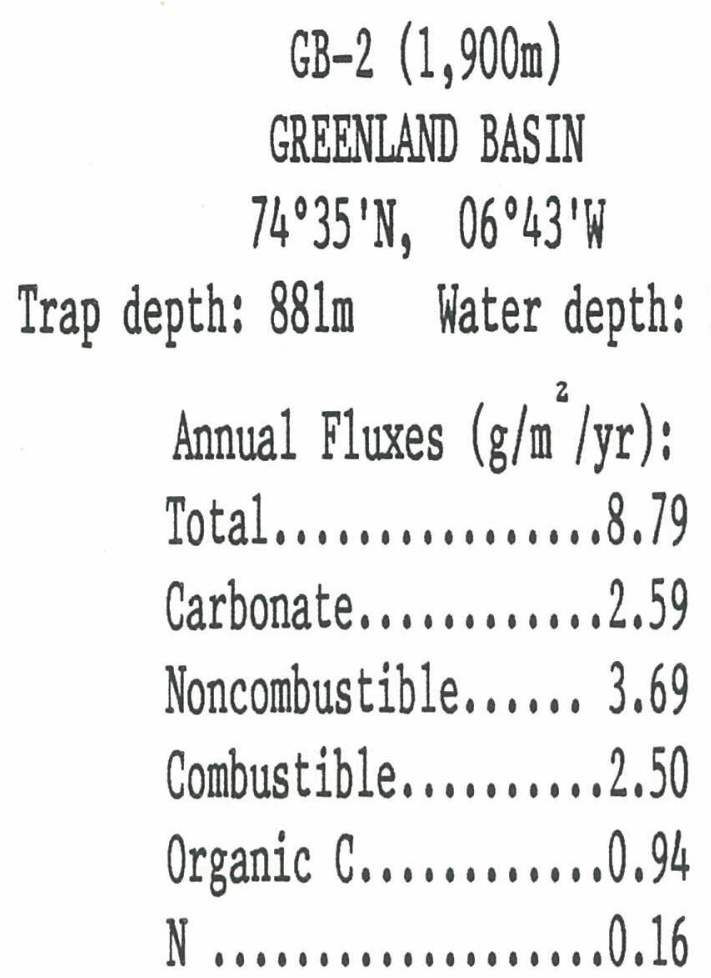

FARFLUX Mark 5-12

\begin{tabular}{|c|c|c|c|c|}
\hline $\begin{array}{l}\text { Sample } \\
\text { ID }\end{array}$ & $\begin{array}{l}\text { Dpening } \\
\text { Date }\end{array}$ & $\begin{array}{l}\text { Closing } \\
\text { Date }\end{array}$ & Span & $\begin{array}{l}\text { Mid. } \\
\text { Date }\end{array}$ \\
\hline $896 B 2-1000-1$ & $0 G-A \cup G-85$ & $95-5 E P-85$ & 30 & $21-A \cup G-85$ \\
\hline $90 \mathrm{GBZ}-1000-2$ & $05-S E P-85$ & $05-0 C T-85$ & 30 & $20-5 E P-85$ \\
\hline $91 \mathrm{GBZ}-1000-3$ & $05-0 C T-85$ & 04-NOU-85 & 30 & $20-0 C T-85$ \\
\hline 92 GB2-1000-4 & $04-N O U-85$ & $04-D E C-85$ & 30 & $19-N O U-85$ \\
\hline $93 \mathrm{~GB} 2-1000-5$ & $04-D E C-85$ & $03-J A N-86$ & 30 & $19-D E C-85$ \\
\hline $94 \mathrm{~GB} 2-1000-6$ & $\emptyset 3-J A N-86$ & $02-F E B-86$ & 30 & 18-JAN-86 \\
\hline $95 \mathrm{GBZ}-1000-7$ & $02-F E B-86$ & $04-M A R-86$ & 30 & $17-F E B-86$ \\
\hline $96 \quad G B 2-1000-8$ & 04-MAR-86 & $93-A P R-86$ & 30 & 19-MAR-86 \\
\hline $97 \mathrm{~GB} 2-1000-9$ & $03-A P R-86$ & $93-M A Y-86$ & 30 & 18-APR-86 \\
\hline $58 \quad G B 2-1000-10$ & $\emptyset 3-M A Y-86$ & $02-J U N-86$ & 30 & 18-MAY-86 \\
\hline $99 \mathrm{~GB}-1000-11$ & $02-J \cup N-86$ & $02-J U L-86$ & 30 & $17-J \cup N-86$ \\
\hline $100 \mathrm{~GB} 2-1000-12$ & $02-J U L-86$ & $01-A \cup G-8 G$ & 30 & $17-J \cup L-86$ \\
\hline
\end{tabular}


Total Flux at Greenland Basin 2, 1900m, 1985-86

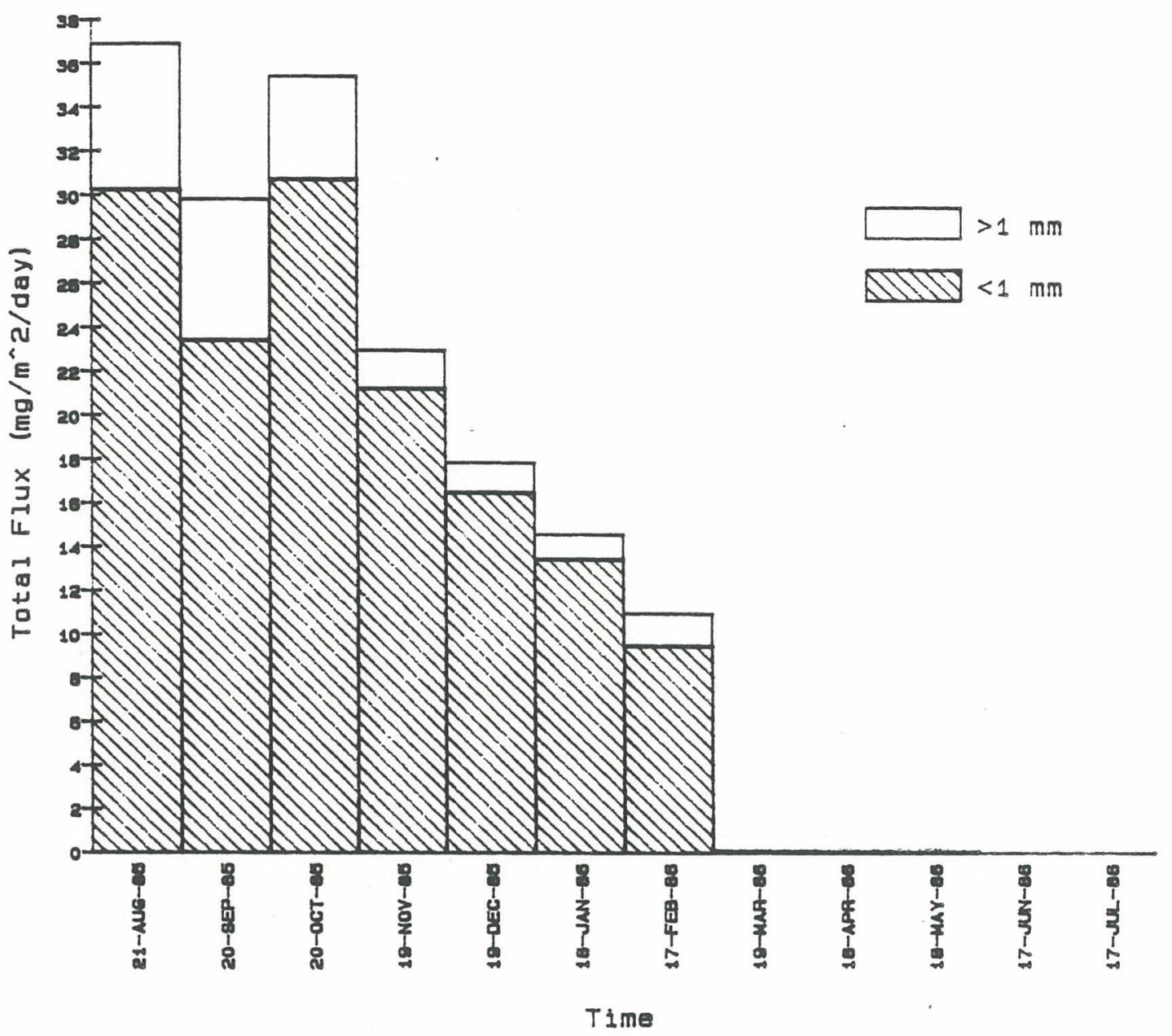

\begin{tabular}{|c|c|c|c|c|c|}
\hline $\begin{array}{l}\text { Sample } \\
\text { I.D. }\end{array}$ & $\begin{array}{l}\text { TTLF } \\
\vdots 1\end{array}$ & $\begin{array}{l}<1 \% \text { of } \\
\text { total }\end{array}$ & $\begin{array}{l}\text { TTLF } \\
>1\end{array}$ & $\begin{array}{l}>1 \% \text { of } \\
\text { total }\end{array}$ & $\begin{array}{l}\text { TTLF } \\
\text { total }\end{array}$ \\
\hline $89 G B 2-1900-1$ & 30.25 & 82.01 & 6.63 & 17.99 & 36.88 \\
\hline $90 \quad 6 B 2-1900-2$ & 23.44 & 78.53 & 6. 41 & 21.47 & 29.86 \\
\hline $91 \mathrm{GBZ}-1900-3$ & 30.75 & 86.77 & 4.69 & 13.23 & 35.44 \\
\hline $92 \quad G B 2-1900-4$ & 21.21 & 92.38 & 1.75 & 7.52 & 22.96 \\
\hline $93 \mathrm{GBZ}-1900-5$ & 16.44 & 92.42 & 1.35 & 7.58 & 17.79 \\
\hline $94 G B 2-1900-6$ & 13.42 & 92.27 & 1.12 & 7.73 & 14.54 \\
\hline $956 B 2-1900-7$ & 9.46 & 86.77 & 1.44 & 13.23 & 10.91 \\
\hline $96 \quad G B 2-1900-8$ & 0.10 & 100.00 & & & 0.10 \\
\hline 97 GBZ-1900-9 & 0.10 & 100.00 & & & 0.10 \\
\hline $98 G B 2-1900-10$ & 0.08 & 100.00. & & & 0.08 \\
\hline $996 B 2-1900-11$ & & & & & \\
\hline 100 GB2-1900-12 & & & & & \\
\hline
\end{tabular}


Carbonate Flux at Greenland Basin 2, 1900m, 1985-86

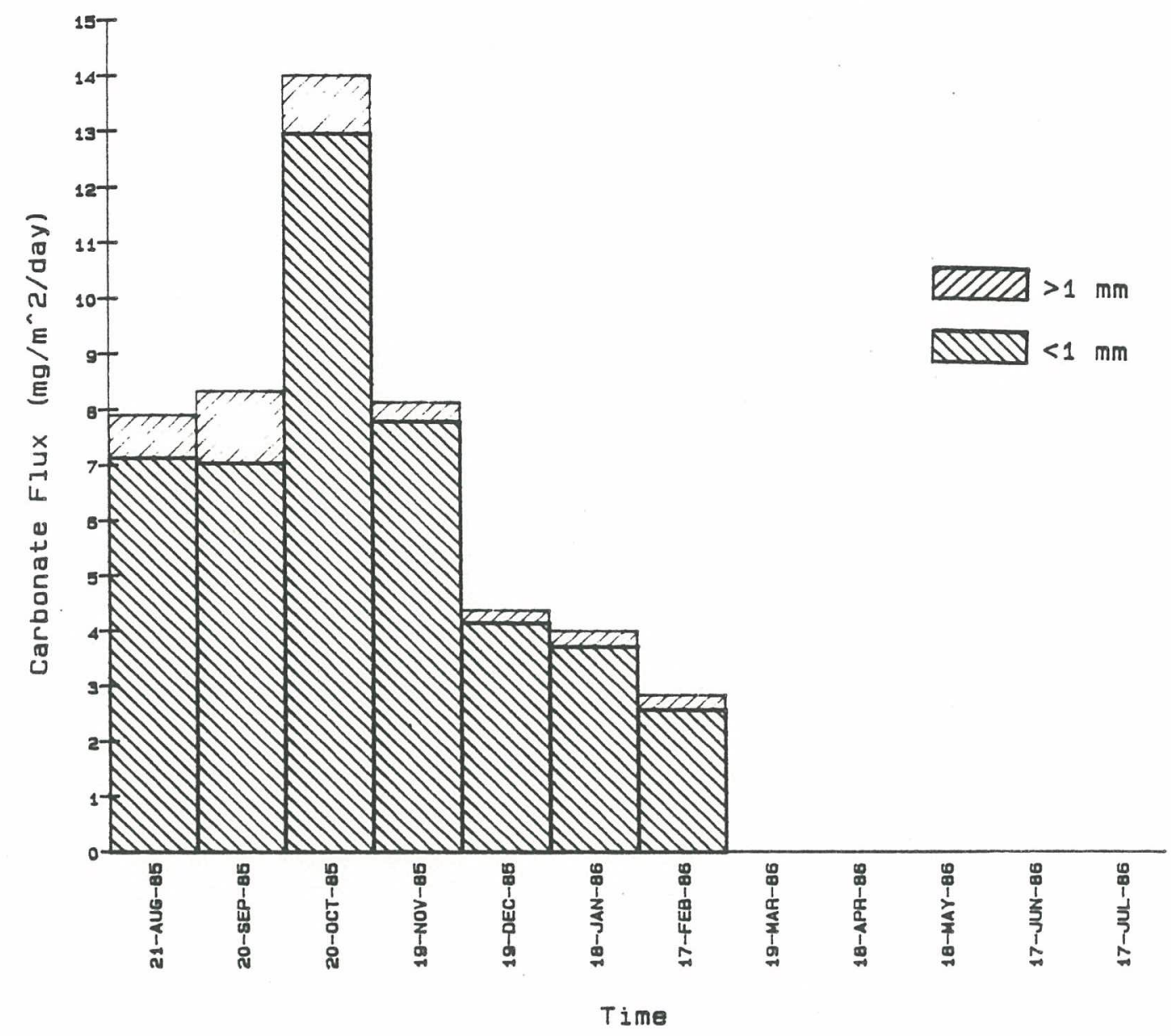

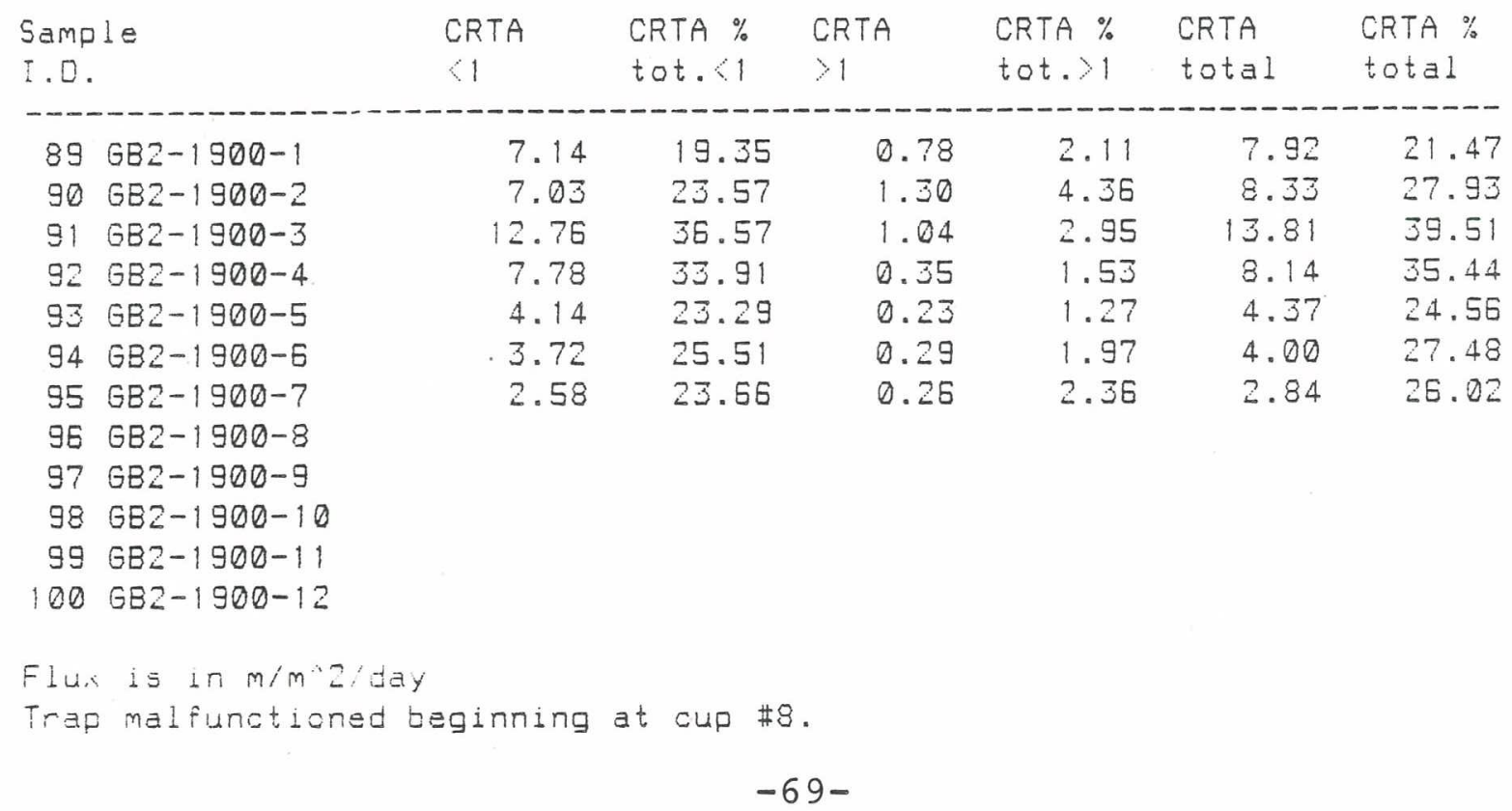


Noncombustible Flux at Greenland Basin 2. 1900m. 1985-86

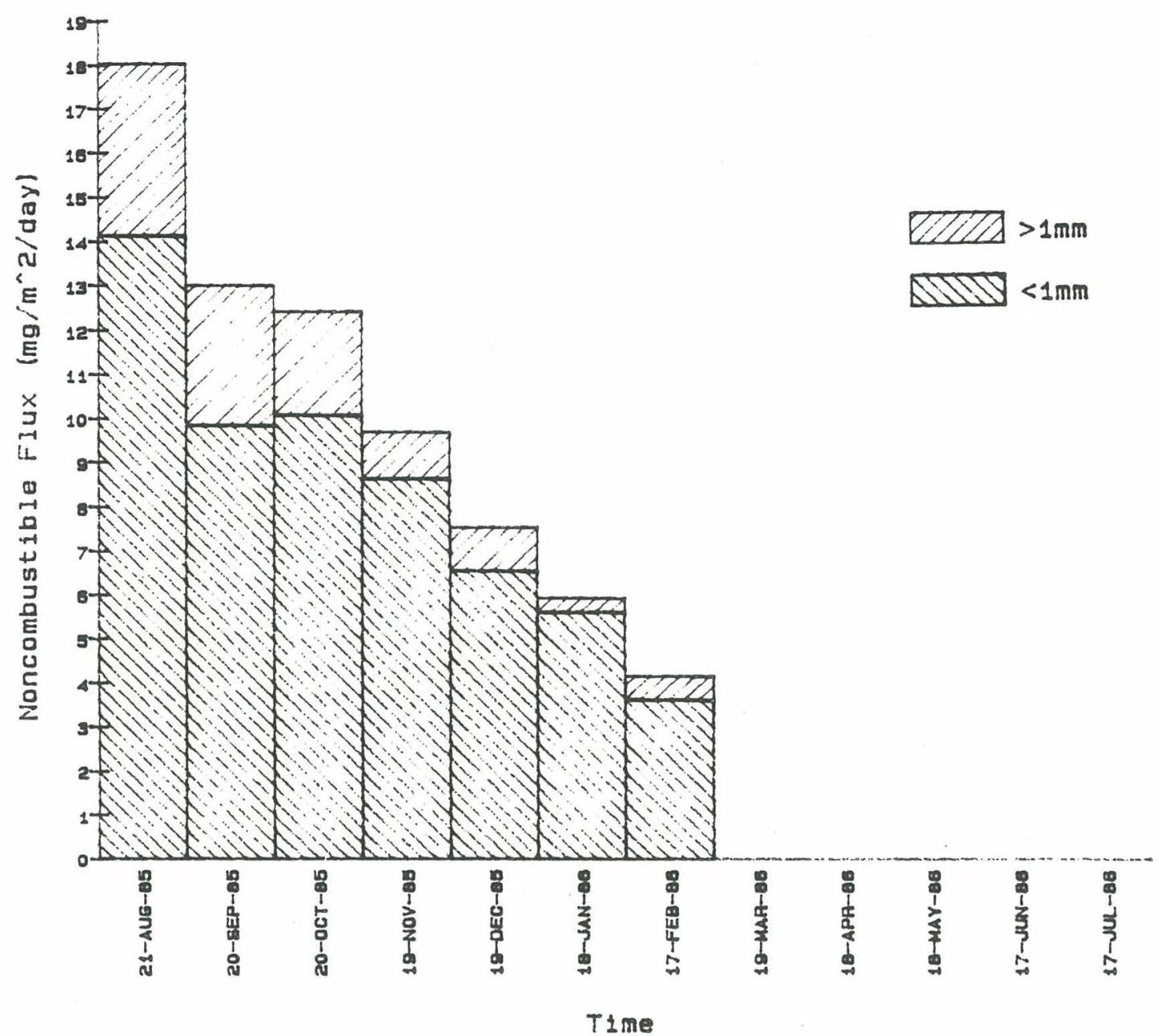

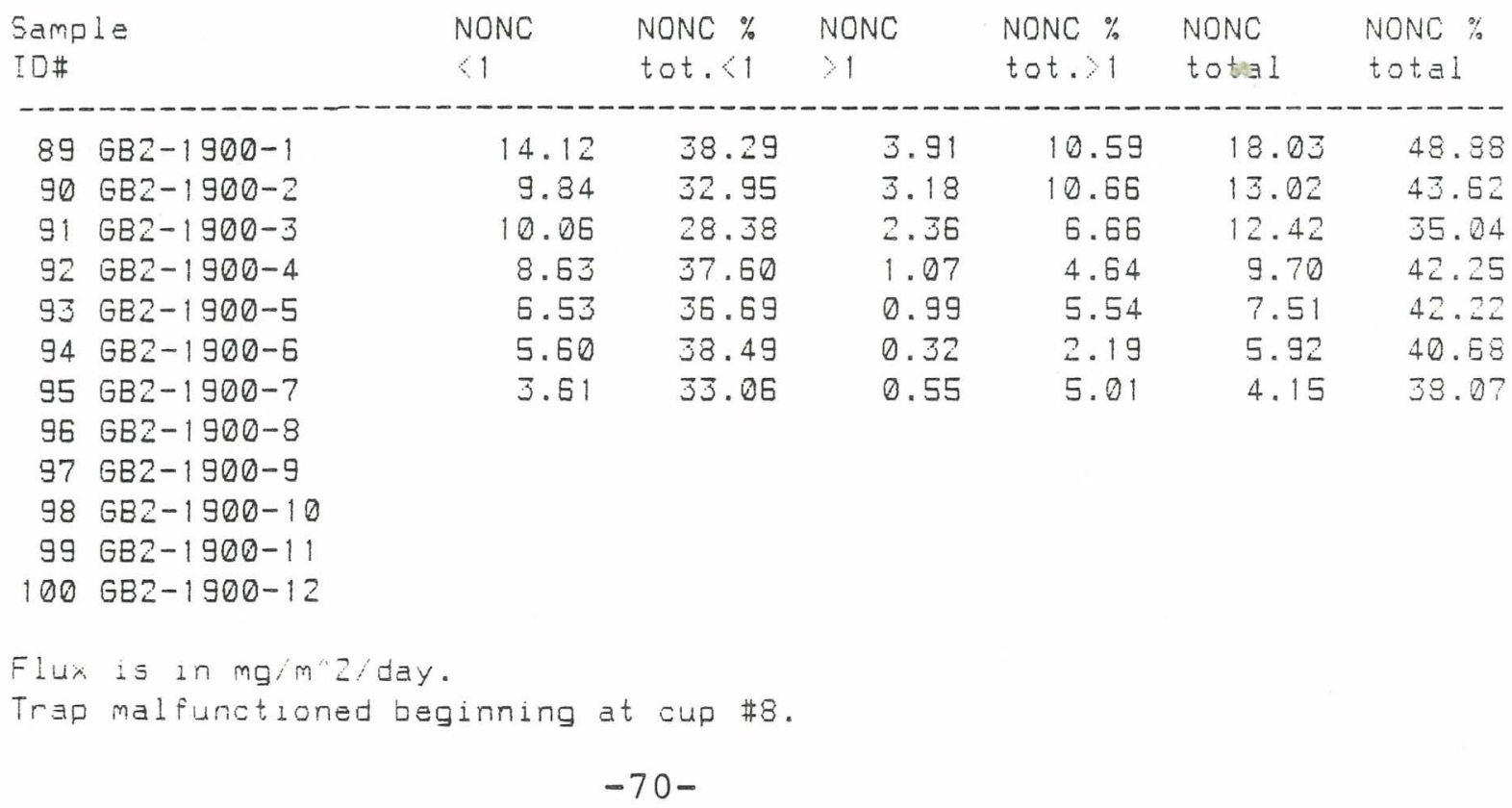


Combustible Flux at Greenland Basin 2. 1900m. 1985-86

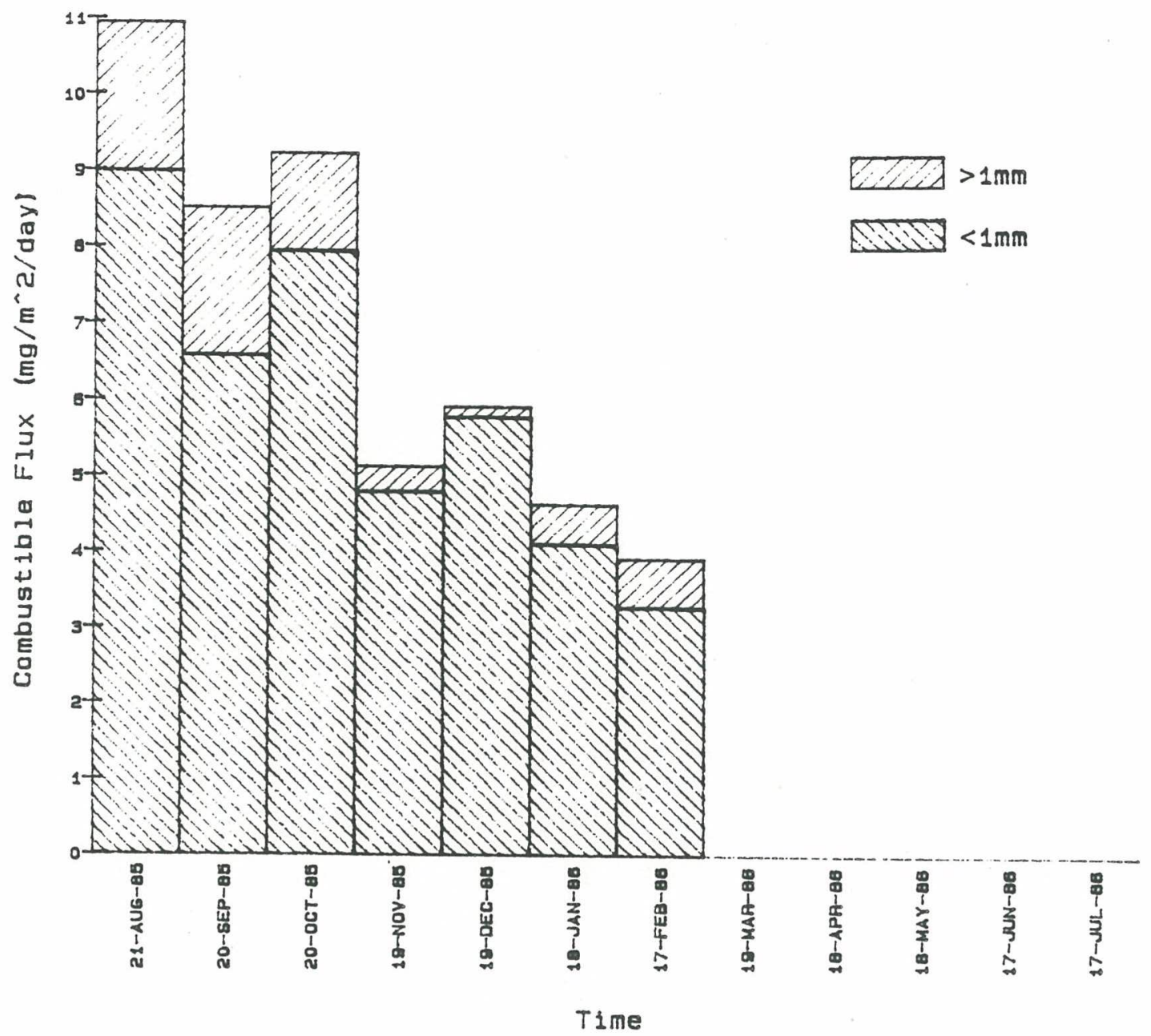

\begin{tabular}{|c|c|c|c|c|c|c|}
\hline $\begin{array}{l}\text { Eample } \\
\text { ID\# }\end{array}$ & $\begin{array}{l}\text { COMB } \\
1\end{array}$ & $\begin{array}{l}\text { COMB } \% \\
\text { tot. } 1\end{array}$ & $\begin{array}{l}\text { COMB } \\
>1\end{array}$ & $\begin{array}{l}\text { COMB } \% \\
\text { tot. } .1\end{array}$ & $\begin{array}{l}\text { COME } \\
\text { TOTAL }\end{array}$ & $\begin{array}{l}\text { Come } \% \\
\text { total }\end{array}$ \\
\hline 89 GEZ-1900-1 & 8.99 & 24.37 & 1.95 & 5.28 & 10.94 & 29.65 \\
\hline 90 GEZ - $1900-2$ & 6.57 & 22.01 & 1.93 & 6.46 & 8.50 & 28.47 \\
\hline $91 G B 2-1900-3$ & 7.93 & 22.38 & 1.29 & 3.63 & 9.22 & 25.01 \\
\hline $926 B 2-1900-4$ & 4.79 & 20.87 & 0.33 & 1.45 & 5.12 & 22.32 \\
\hline $93682-1900-5$ & 5.77 & 32.44 & 0.14 & 0.78 & 5.91 & 33.22 \\
\hline $946 B 2-1900-6$ & 4.10 & 28.22 & 0.52 & 3.57 & 4.62 & 31.79 \\
\hline 95 GE2-1900-7 & 3.27 & 30.02 & 0.64 & 5.86 & 3.91 & 35.98 \\
\hline $96 \quad G B 2-1900-8$ & & & & & & \\
\hline $97 \mathrm{~GB} 2-1900-9$ & & & & & & \\
\hline $98 \quad 6 B 2-1900-10$ & & & & & & \\
\hline $996 B 2-1900-11$ & & & & & & \\
\hline $100 \quad 6 B 2-1900-12$ & & & & & & \\
\hline
\end{tabular}


Carbon Flux at Greenland Basin 2. 1900m. 1985-86

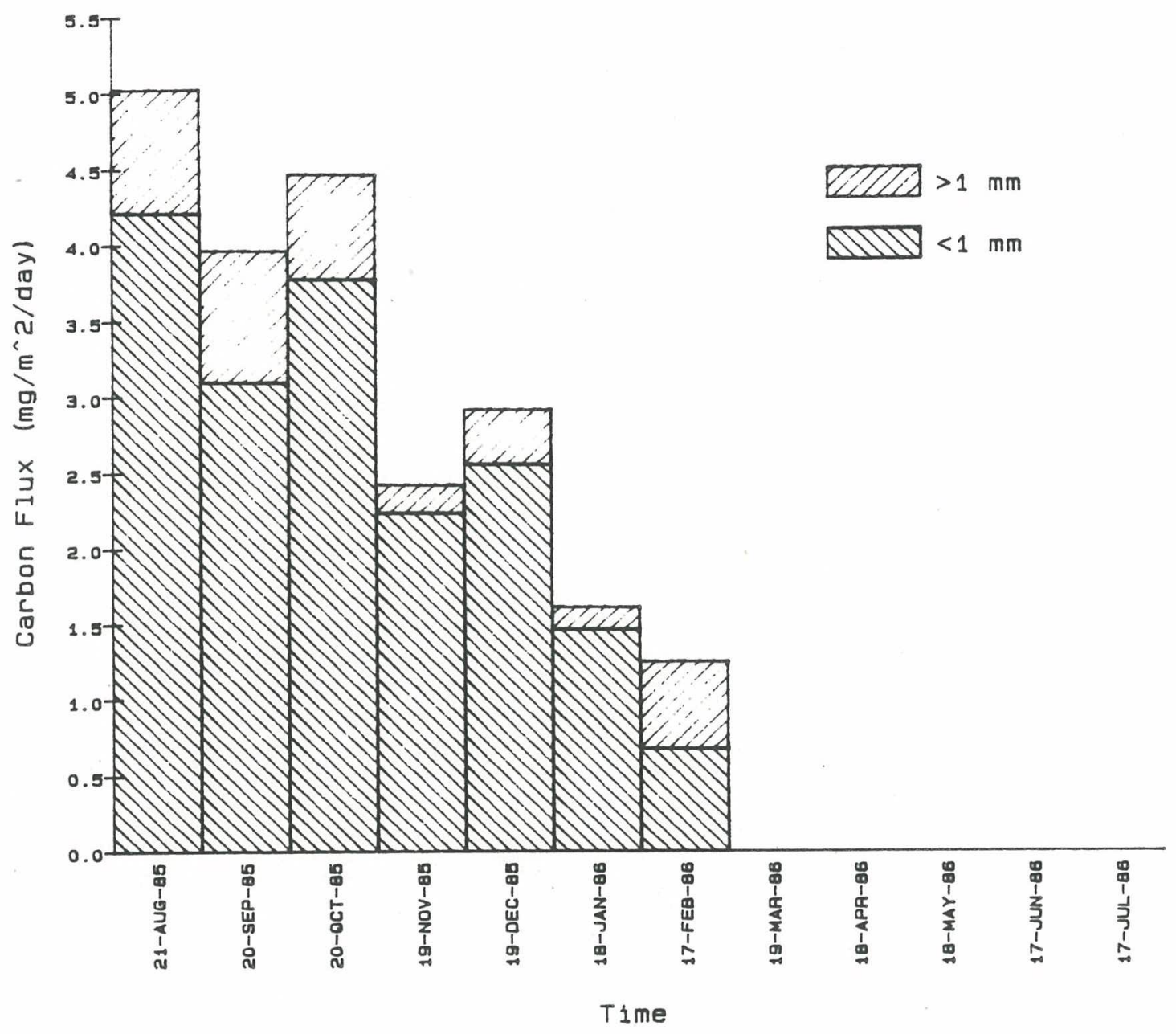

\begin{tabular}{|c|c|c|c|c|c|c|}
\hline $\begin{array}{l}\text { Sample } \\
\text { I.D. }\end{array}$ & $\begin{array}{l}\text { CRNC } \\
<1\end{array}$ & $\begin{array}{l}\text { CRNC }<1 \\
\% \text { cmb } f .\end{array}$ & $\begin{array}{l}\text { CRNC } \\
>1\end{array}$ & $\begin{array}{l}\text { CRNC> } 1 \\
\% \text { cmbf. }\end{array}$ & $\begin{array}{l}\text { CRNC } \\
\text { total }\end{array}$ & $\begin{array}{l}\text { CRNCtot. } \\
\text { \%cmb } f .\end{array}$ \\
\hline $89 G B 2-1900-1$ & 4.21 & 38.53 & 0.82 & 7.46 & 5.03 & 45.98 \\
\hline $906 B 2-1900-2$ & 3.10 & 36.43 & 0.87 & 10.21 & 3.97 & 46. \\
\hline $916 B 2-1900-3$ & 3.78 & 41.02 & 0.69 & 7.49 & 4.47 & 48. \\
\hline $926 B 2-1900-4$ & 2.24 & 43.70 & 0.19 & 3.65 & 2.43 & 47.4 \\
\hline $936 B 2-1900-5$ & 2.56 & 43.32 & 0.36 & 6.15 & 2.92 & 49. \\
\hline $94 G B 2-1900-6$ & 1.48 & 32.00 & 0.15 & 3.28 & 1.63 & 35.2 \\
\hline $95 \mathrm{~GB} 2-1900-7$ & 0.68 & 17.49 & 0.57 & 14.68 & 1.25 & 31.5 \\
\hline $96 \quad G B 2-1900-8$ & & & & & & \\
\hline $976 B 2-1900-9$ & & & & & & \\
\hline $98 \quad 6 B 2-1900-10$ & & & & & & \\
\hline $99 G B 2-190 \theta-11$ & & & & & & \\
\hline 100 GB2-1900-12 & & & & & & \\
\hline
\end{tabular}


Nitrogen Flux at Greenland Basin 2. 1900m, 1985-86

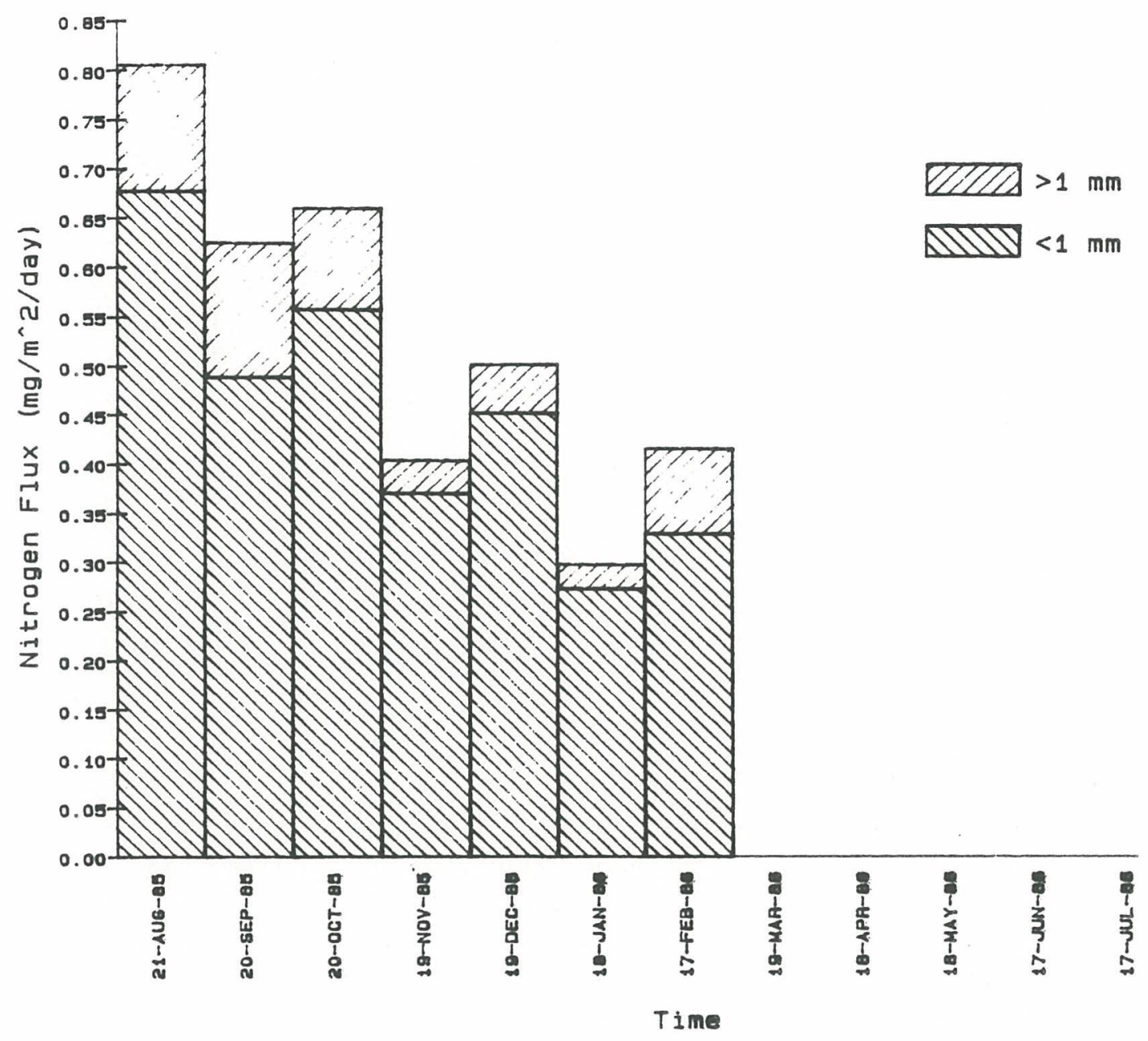

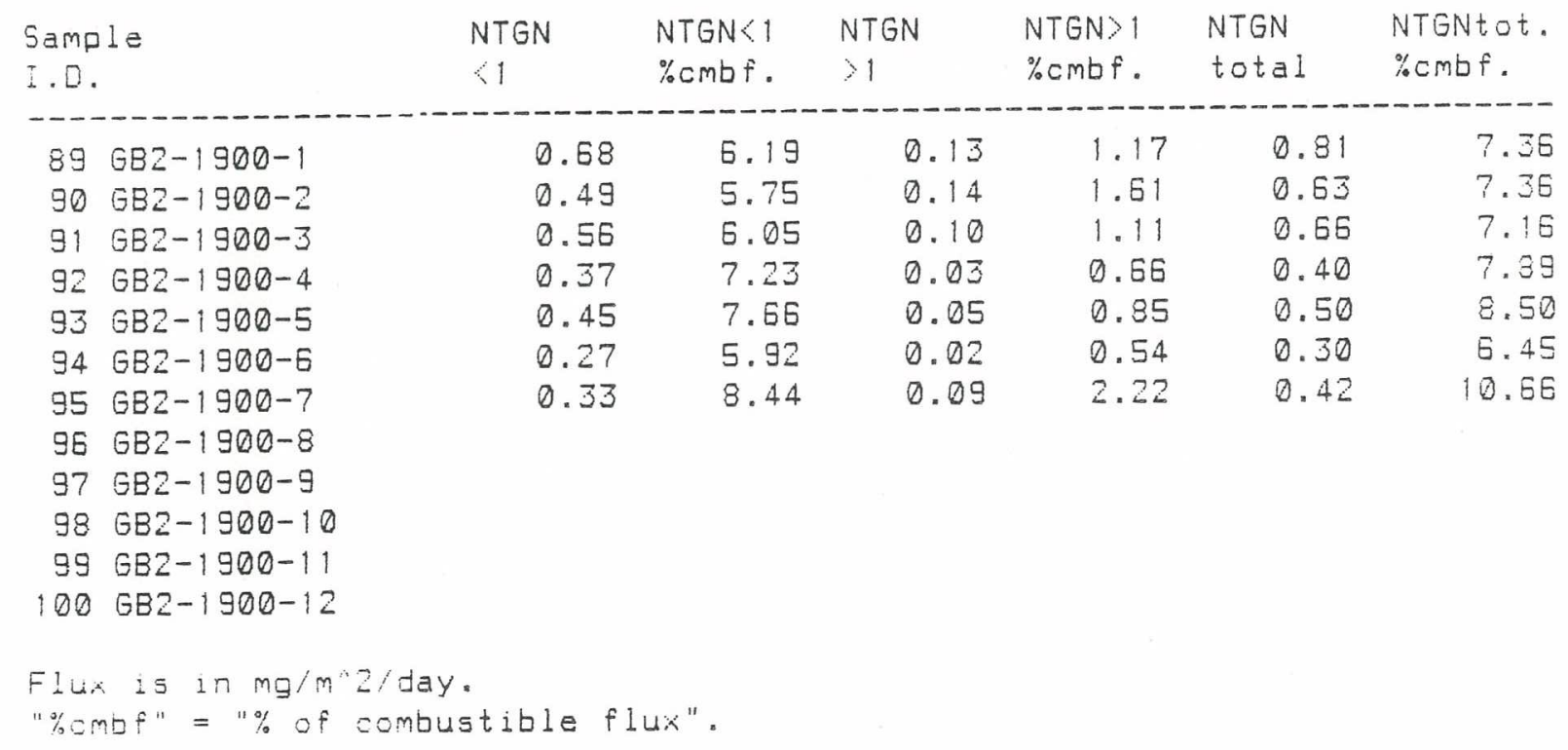


Hydrogen Flux at Greenland Basin 2. 1900m. 1985-86

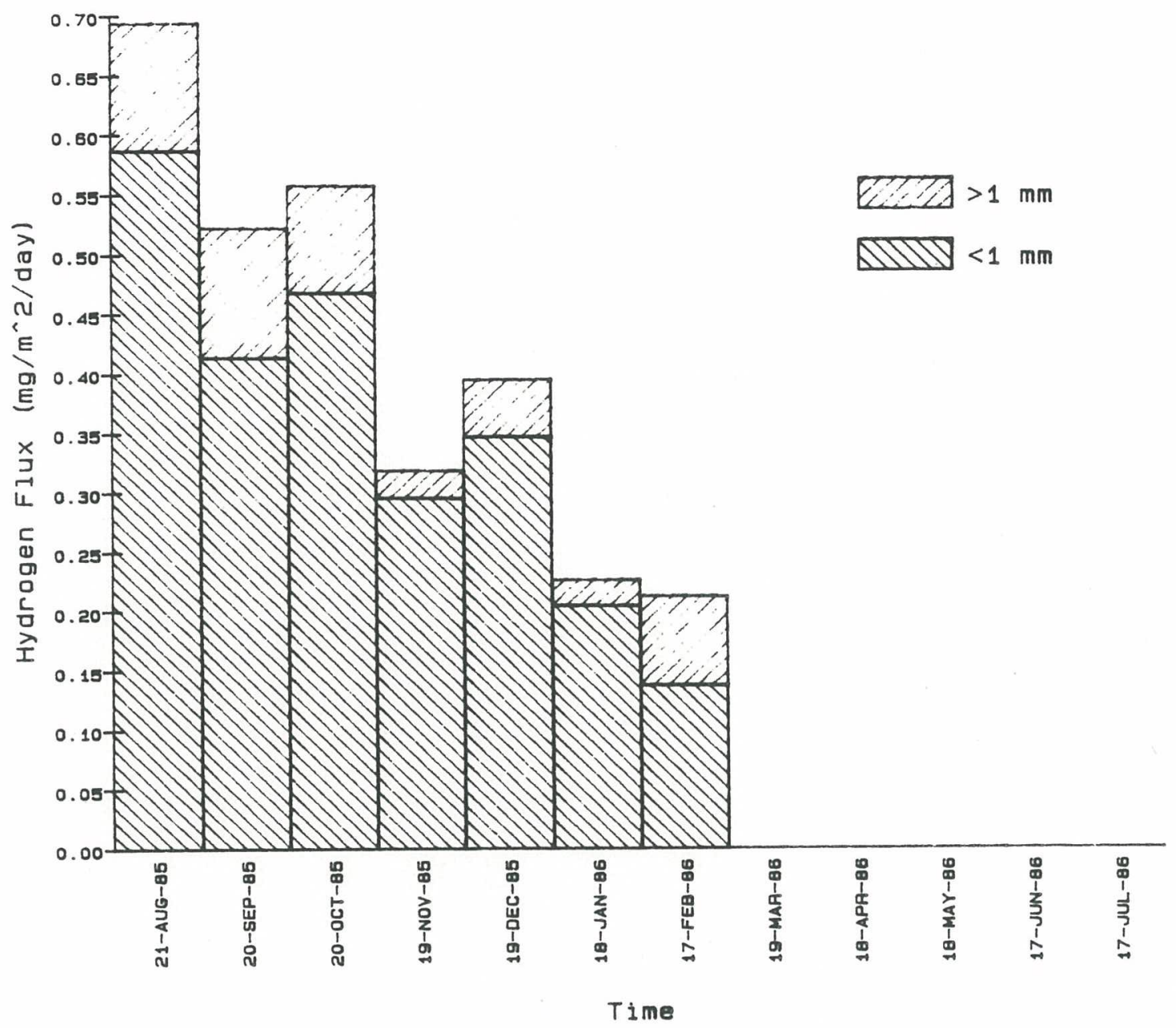

\begin{tabular}{|c|c|c|c|c|c|c|}
\hline $\begin{array}{l}\text { Sample } \\
\text { I.D. }\end{array}$ & $\begin{array}{l}\text { HYDC } \\
<1\end{array}$ & $\begin{array}{l}\text { HYDC } 1 \\
\% \text { cmb f. }\end{array}$ & $\begin{array}{l}\text { HYDC } \\
>1\end{array}$ & $\begin{array}{l}\text { HYDC }>1 \\
\% c M b f .\end{array}$ & $\begin{array}{l}\text { HYDC } \\
\text { total }\end{array}$ & $\begin{array}{l}\text { HYDCtot. } \\
\% \text { cmb f. }\end{array}$ \\
\hline $89 G B 2-1900-1$ & 0.59 & 5.37 & 0.11 & 0.98 & 0.59 & 6.35 \\
\hline $90 G B 2-1900-2$ & 0.41 & 4.87 & 0.11 & 1.27 & 0.52 & 6. 14 \\
\hline $91 G B 2-1900-3$ & 0.47 & 5.07 & 0.09 & 0.98 & 0.56 & 6.05 \\
\hline $92 \mathrm{~GB} 2-1900-4$ & 0.30 & 5.76 & 0.02 & 0.45 & 0.32 & 6.21 \\
\hline $93 G B 2-1900-5$ & 0.35 & 5.87 & 0.05 & 0.82 & 0.40 & 6.69 \\
\hline $94 G E 2-1900-6$ & 0.20 & 4.43 & 0.02 & 0.47 & 0.23 & 4.89 \\
\hline $95 G B 2-1900-7$ & 0.14 & 3.52 & 0.08 & 1.92 & 0.21 & 5.44 \\
\hline $96 \quad G B 2-1900-8$ & & & & & & \\
\hline $97 G B 2-1900-9$ & & & & & & \\
\hline $98 \quad G B 2-1900-10$ & & & & & & \\
\hline $99 \mathrm{~GB} 2-1900-11$ & & & & & & \\
\hline $100 \mathrm{~GB} 2-1900-12$ & & & & & & \\
\hline
\end{tabular}




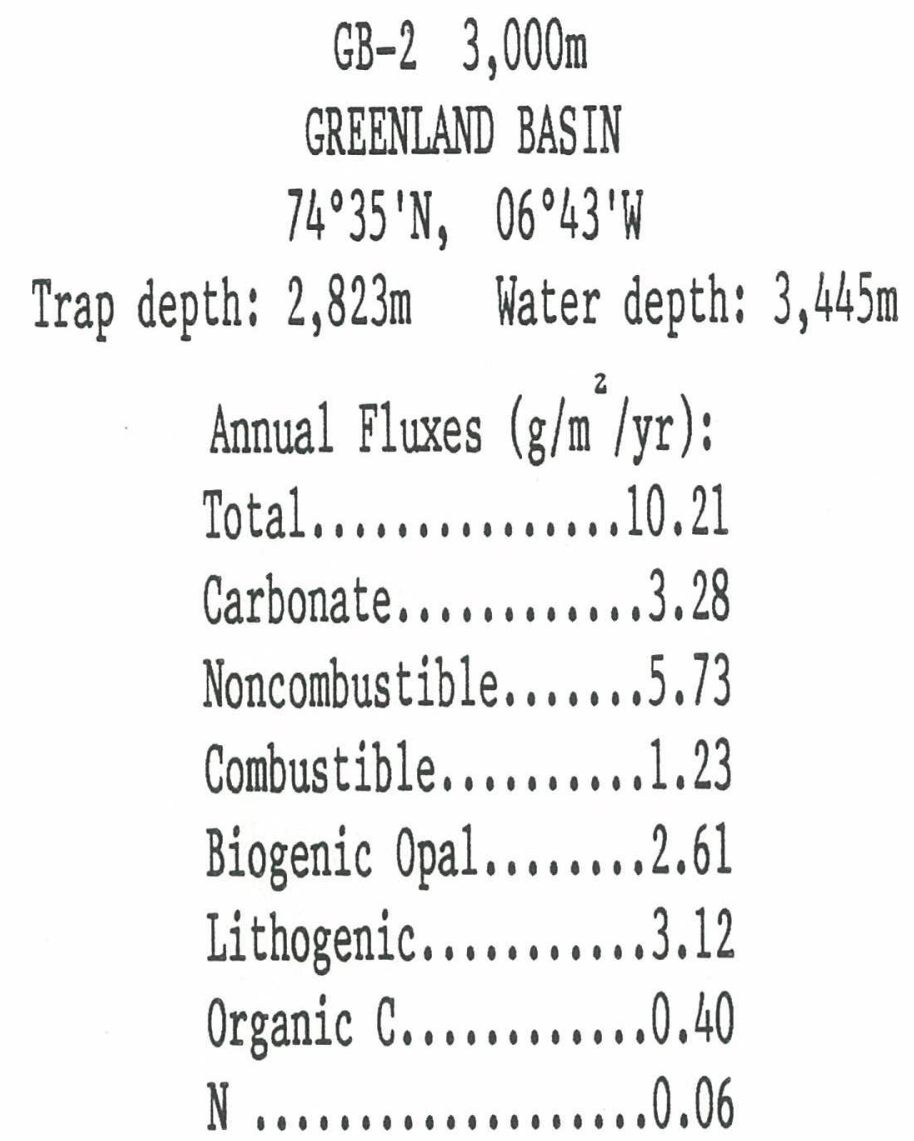

PARFLUX Mark 6-13

\begin{tabular}{|c|c|c|c|c|}
\hline $\begin{array}{l}\text { Sample } \\
\text { ID }\end{array}$ & $\begin{array}{l}\text { Opening } \\
\text { Date }\end{array}$ & $\begin{array}{l}\text { Closing } \\
\text { Date }\end{array}$ & Span & $\begin{array}{l}\text { Mid. } \\
\text { Date }\end{array}$ \\
\hline $101 \mathrm{GB2}-3000-1$ & $04-A \cup G-85$ & $01-5 E P-85$ & 28 & $18-A \cup G-85$ \\
\hline 102 GB2-3000-2 & $01-5 E P-85$ & 29-5EP-85 & 28 & $15-S E P-85$ \\
\hline $103 \quad 6 B 2-3000-3$ & $29-5 E P-85$ & $27-0 C T-85$ & 28 & $13-0 C T-85$ \\
\hline 104 GB2-3000-4 & $27-00 T-85$ & $24-$ NOU -85 & 28 & $10-N O V-85$ \\
\hline $105 \mathrm{GBZ}-3000-5$ & $24-\mathrm{NOU}-85$ & $22-D E C-85$ & 28 & $08-0 E C-85$ \\
\hline 106 GB2-3000-6 & $22-D E C-85$ & $19-J A N-8 E$ & 28 & $95-J A N-86$ \\
\hline $107 \mathrm{GBZ}-3000-7$ & $19-J A N-86$ & $1 E-F E B-86$ & 28 & $\theta_{2}-F E B-86$ \\
\hline 108 GBZ-3000-8 & $16-F E B-86$ & $16-M A R-86$ & 28 & $02-M A R-86$ \\
\hline 109 GB2-3000-9 & 16-MAR-86 & $13-A P R-86$ & 28 & $30-M A R-86$ \\
\hline $110 \mathrm{~GB} 2-3000-10$ & $13-A P R-8 E$ & $11-M A Y-86$ & 28 & $27-A P R-86$ \\
\hline $1116 \mathrm{~GB} 2-3000-11$ & $11-M A Y-86$ & $\square 8-J U N-86$ & 28 & $25-M A Y-86$ \\
\hline 112 GB2-3000-12 & $08-J \cup N-8 E$ & $D E-J U L-B E$ & 28 & $22-J U N-8 E$ \\
\hline 113 GB2-3000-13 & $06-J U L-86$ & $03-A \cup G-86$ & 28 & $20-J U L-86$ \\
\hline
\end{tabular}


Total Flux at Greenland Basin 2. 3000m, 1985-86

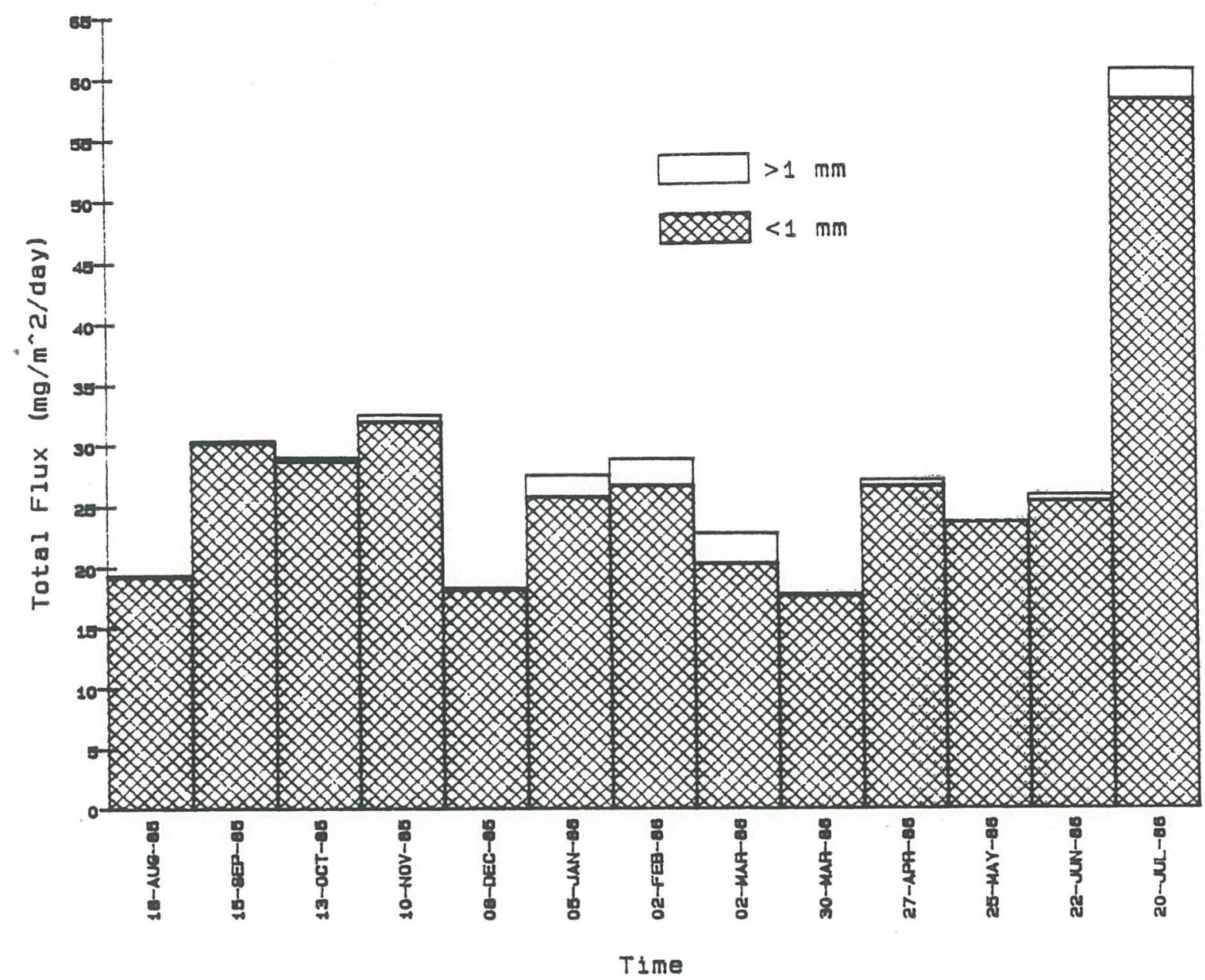

\begin{tabular}{|c|c|c|c|c|c|}
\hline $\begin{array}{l}\text { Sample } \\
\text { I.D. }\end{array}$ & $\begin{array}{l}\text { TTLF } \\
\langle 1\end{array}$ & $\begin{array}{l}<1 \% \text { of } \\
\text { total }\end{array}$ & $\begin{array}{l}\text { TTLF } \\
>1\end{array}$ & $\begin{array}{l}>1 \% \text { of } \\
\text { total }\end{array}$ & $\begin{array}{l}\text { TTLF } \\
\text { total }\end{array}$ \\
\hline 10$\} \mathrm{GB} 2-3000-1$ & 19.29 & 99.48 & 0.10 & 0.52 & 19.39 \\
\hline $102 \mathrm{~GB} 2-3000-2$ & 30.23 & 99.43 & 0.17 & 0.57 & 30.40 \\
\hline $1036 B 2-3000-3$ & 28.68 & 98.94 & 0.31 & 1.06 & 28.99 \\
\hline 104 GB2-3000-4 & 31.92 & 98.34 & 0.54 & 1.66 & 32.46 \\
\hline $105 \mathrm{~GB} 2-3000-5$ & 18.15 & 99.37 & 0.11 & 0.63 & 18.27 \\
\hline $106 \quad 6 B 2-3000-6$ & 25.71 & 93.48 & 1.79 & 6.52 & 27.51 \\
\hline $107 \quad 6 B 2-3000-7$ & 26.64 & 92.41 & 2.19 & 7.59 & 28.83 \\
\hline $108 \quad 6 B 2-3000-8$ & 20.24 & 89.10 & 2.48 & 10.90 & 22.71 \\
\hline $109682-3000-9$ & 17.57 & 99.26 & 0.13 & 6.74 & 17.70 \\
\hline $110682-3000-10$ & 26.59 & 98.12 & 0.51 & 1.88 & 27.10 \\
\hline $111 G B 2-3000-11$ & 23.64 & 100.00 & & & 23.54 \\
\hline 112 GB2-3000-12 & 25.28 & 97.96 & 0.53 & 2.04 & 25.80 \\
\hline $113 G B 2-3000-13$ & 58.24 & 96.00 & 2.43 & 4.00 & 50.67 \\
\hline
\end{tabular}

Flux is in $m g / m^{\prime \prime} 2 /$ day. 
Carbonate Flux at Greenland Basin 2. 3000m, 1985-86

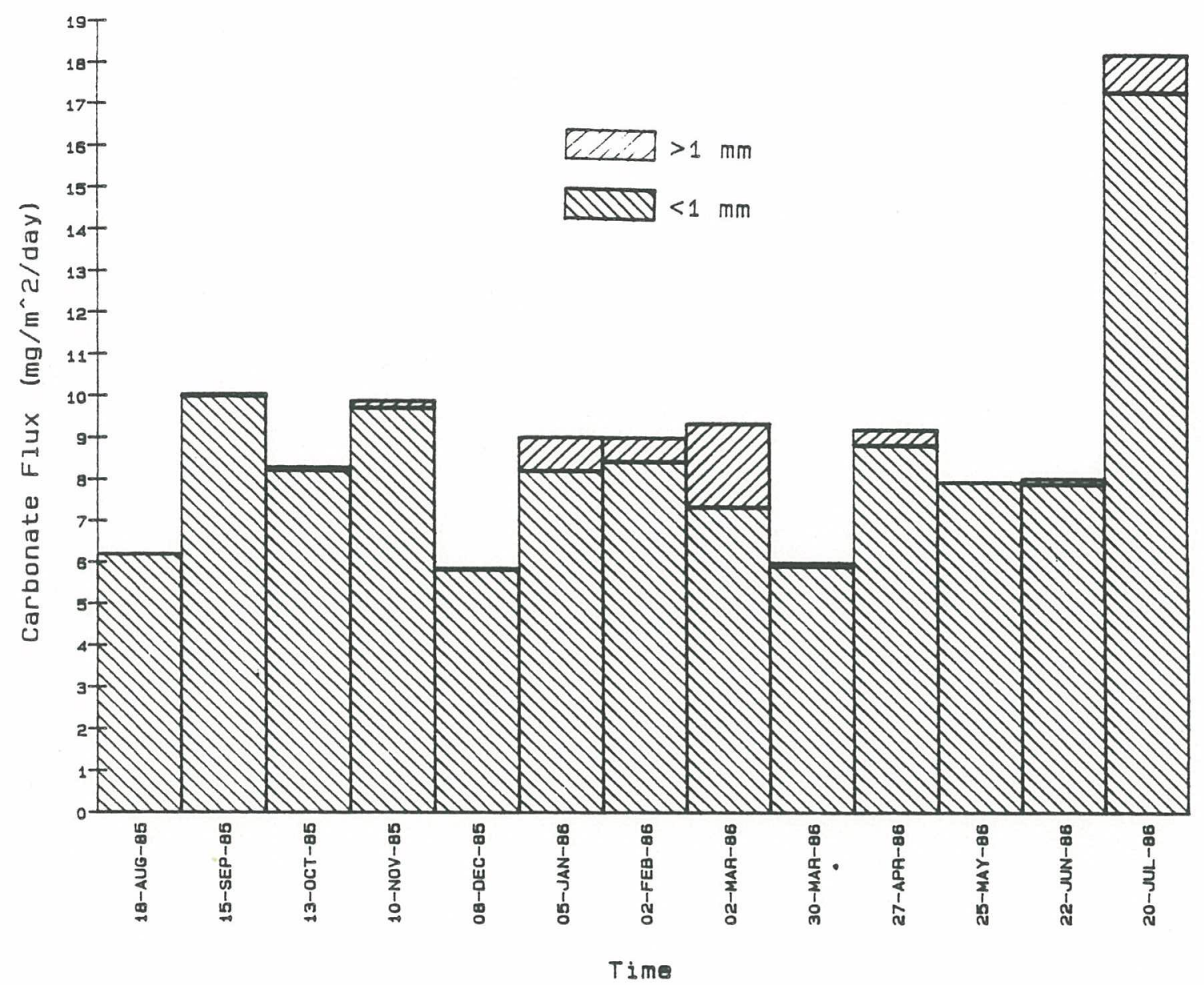

\begin{tabular}{|c|c|c|c|c|c|c|}
\hline $\begin{array}{l}\text { Sample } \\
\text { I.D. }\end{array}$ & $\begin{array}{l}\text { CRTA } \\
<1\end{array}$ & $\begin{array}{l}\text { CRTA } \% \\
\text { tot. }<1\end{array}$ & $\begin{array}{l}\text { CRTA } \\
>1\end{array}$ & $\begin{array}{l}\text { CRTA } \% \\
\text { tot. }>1\end{array}$ & $\begin{array}{l}\text { CRTA } \\
\text { total }\end{array}$ & $\begin{array}{l}\text { CRTA } \% \\
\text { total }\end{array}$ \\
\hline $1016 B 2-3000-1$ & 6.19 & 31.93 & 0.00 & 31.93 & 6.20 & 31. \\
\hline $102682-3000-2$ & 10.01 & 32.91 & 0.04 & 32.91 & 10.04 & 33. \\
\hline $103682-3000-3$ & 8.20 & 28.30 & 0.06 & 28.30 & 8.27 & 28. \\
\hline $104682-3000-4$ & 9.70 & 29.90 & 0.17 & 29.90 & 9.87 & 30. \\
\hline $105682-3000-5$ & 5.81 & 31.80 & 0.00 & 31.80 & 5.81 & 31.83 \\
\hline $106 \quad G B 2-3000-6$ & 8.20 & 29.82 & 0.26 & 29.82 & 8.46 & 30.7 \\
\hline $107682-3000-7$ & 8.42 & 29.20 & 0.56 & 29.20 & 8.98 & 31.1 \\
\hline $108 \quad 682-3000-8$ & 7.33 & 32.25 & 1.44 & 32.25 & 8.77 & 38.5 \\
\hline $1096 B 2-3000-9$ & 5.90 & 33.35 & 0.07 & 33.35 & 5.98 & 33.7 \\
\hline 110 GE2-3000-10 & 8.80 & 32.48 & 0.10 & 32.48 & 8.90 & 32.8 \\
\hline $1116 E 2-3000-11$ & 7.94 & 33.59 & & & 7.94 & 33.59 \\
\hline $112682-3000$ & 7.89 & 30.56 & 0.13 & 30.56 & 8.02 & 31.07 \\
\hline $1136 B 2-3000$ & 17.30 & 28.51 & 0.42 & 28.51 & 17.72 & 29.2 \\
\hline
\end{tabular}

Flux is in mg/m" $2 /$ day. 
Noncombustible Flux, at Greenland Basin 2. 3000m. 1985-6

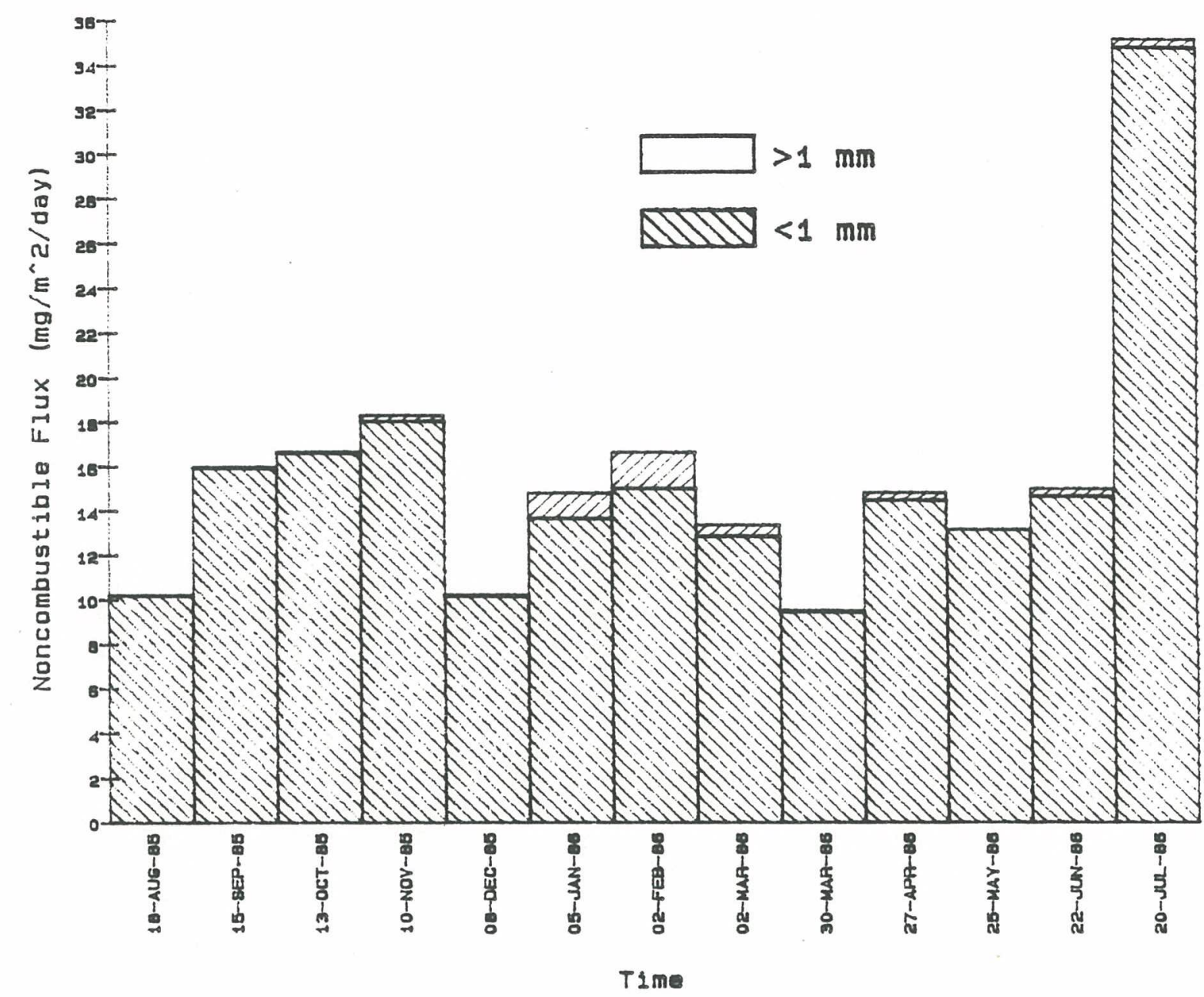

\begin{tabular}{|c|c|c|c|c|c|c|}
\hline $\begin{array}{l}\text { Sample } \\
\text { ID\# }\end{array}$ & $\begin{array}{l}\text { NONC } \\
<1\end{array}$ & $\begin{array}{l}\text { NONC } \% \\
\text { tot. } 1\end{array}$ & $\begin{array}{l}\text { NONC } \\
>1\end{array}$ & $\begin{array}{l}\text { NONC } \% \\
\text { tot. } 1\end{array}$ & $\begin{array}{l}\text { NONC } \\
\text { total }\end{array}$ & $\begin{array}{l}\text { NONC } \% \\
\text { tota } 1\end{array}$ \\
\hline $101 \quad 6 B 2-3000-1$ & 10.19 & 52.55 & 0.04 & 0.20 & 10.23 & 52.75 \\
\hline $1026 B 2-3000-2$ & 15.96 & 52.48 & 0.07 & 0.23 & 16.03 & 52.71 \\
\hline 103 GB2-3000-3 & 16.61 & 57.29 & 0.08 & 0.28 & $1 E . E 9$ & 57.57 \\
\hline 104 GB2-3000-4 & 18.04 & 55.58 & 0.28 & 0.86 & 18.32 & 56.44 \\
\hline 105 GE2-3000-5 & 10.16 & 55.61 & $\emptyset .06$ & 0.30 & 10.21 & 55.91 \\
\hline $106 \quad 6 B 2-3000-6$ & 13.66 & 49.66 & 1.15 & 4.18 & 14.81 & $5 \overline{3} .84$ \\
\hline 107 GB2-3000-7 & 15.04 & 52.15 & 1.62 & 5.63 & 16.65 & 57.78 \\
\hline 108 GE2-3000-8 & 12.86 & 56.62 & 0.49 & 2.18 & 13.35 & 58.79 \\
\hline $109 \mathrm{GBZ}-3000-9$ & 9.44 & 53.32 & 0.06 & 0.33 & 9.50 & 53.65 \\
\hline $110 G B 2-3000-10$ & 14.50 & 53.50 & 0.35 & 1.29 & 14.85 & 54.79 \\
\hline 111 GB2-3000-11 & 13.14 & 55.58 & & & 13.14 & 55.58 \\
\hline 112 GB2-3000-12 & 14.66 & 56.81 & 0.32 & 1.25 & 14.98 & 58.07 \\
\hline $1136 B 2-3000-13$ & 34.76 & 57.30 & 0.38 & 0.62 & 35.14 & 57.92 \\
\hline
\end{tabular}

Flux is in $m g / m^{\prime \prime} /$ day. 
Combustible Flux at Greenland Basin 2. 3000m. 1985-86

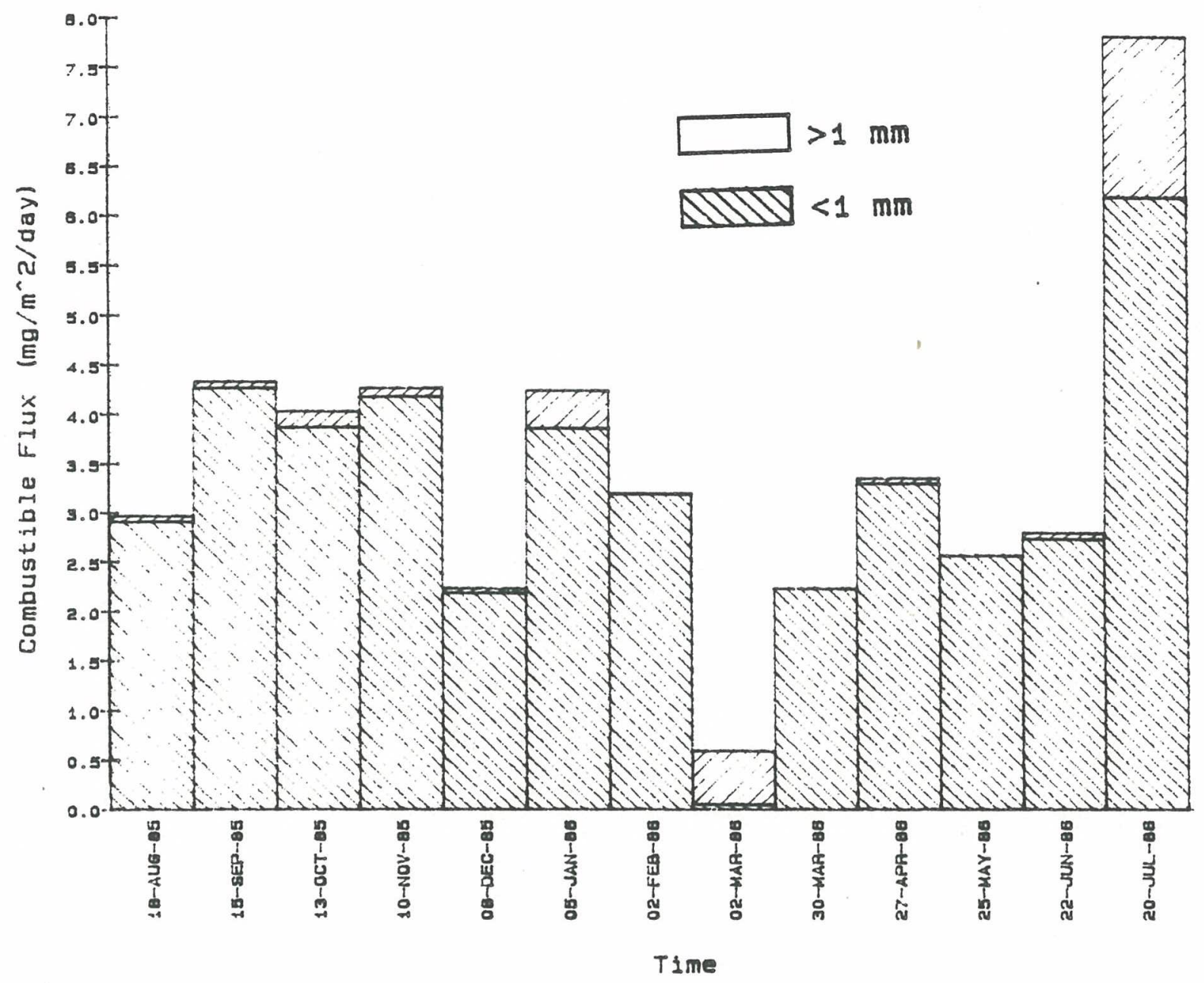

\begin{tabular}{|c|c|c|c|c|c|c|}
\hline $\begin{array}{l}\text { Sample } \\
\text { ID\# }\end{array}$ & $\begin{array}{l}\text { COMB } \\
<1\end{array}$ & $\begin{array}{l}\text { COMB } \% \\
\text { tot. }<1\end{array}$ & $\begin{array}{l}\text { COMB } \\
>1\end{array}$ & $\begin{array}{l}\text { COMB } \% \\
\text { tot. } \% 1\end{array}$ & $\begin{array}{l}\text { COMB } \\
\text { TOTAL }\end{array}$ & $\begin{array}{l}\text { COMB } \% \\
\text { total }\end{array}$ \\
\hline $101 \mathrm{GBZ}-3000-1$ & 2.91 & 15.00 & 0.06 & 0.30 & 2.97 & 15.30 \\
\hline $102 G B 2-3000-2$ & 4.27 & 14.04 & 0.07 & 0.23 & 4.34 & 14.26 \\
\hline 103 GE2-3000-3 & 3.87 & 13.35 & 0.16 & 0.56 & 4.03 & 13.92 \\
\hline $104 \mathrm{~GB} 2-3000-4$ & 4.18 & 12.87 & 0.09 & 0.28 & 4.27 & 13.15 \\
\hline 105 GE2-3000-5 & 2.18 & 11.96 & 0.05 & 0.30 & 2.24 & 12.26 \\
\hline 106 GBZ-3000-6 & 3.85 & 14.01 & 0.39 & 1.40 & 4.24 & 15.41 \\
\hline $107 \mathrm{GBZ}-3000-7$ & 3.19 & 11.06 & 0.01 & 0.02 & 3.20 & 11.08 \\
\hline $108 \quad G B 2-3000-8$ & 0.05 & 0.23 & 0.54 & 2.39 & 0.59 & 2.61 \\
\hline $1096 B 2-3000-9$ & 2.23 & 12.59 & 0.00 & 0.00 & 2.23 & 12.59 \\
\hline $110 \mathrm{~GB} 2-3000-10$ & 3.29 & 12.14 & 0.06 & 0.22 & 3.35 & 12.36 \\
\hline $111 G B 2-3000-11$ & 2.56 & 10.82 & & & 2.56 & 10.82 \\
\hline $112 G B 2-3000-12$ & 2.73 & 10.58 & 0.07 & 0.28 & 2.80 & 10.86 \\
\hline $113 G B 2-3000-13$ & 6.18 & 10.19 & 1.53 & 2.59 & 7.81 & 12.88 \\
\hline
\end{tabular}

Flux is in $m g / m^{\prime \prime 2} /$ day. 
Blogenic Silica Flux at Greenland Basin 2. 3000m. 1985-96

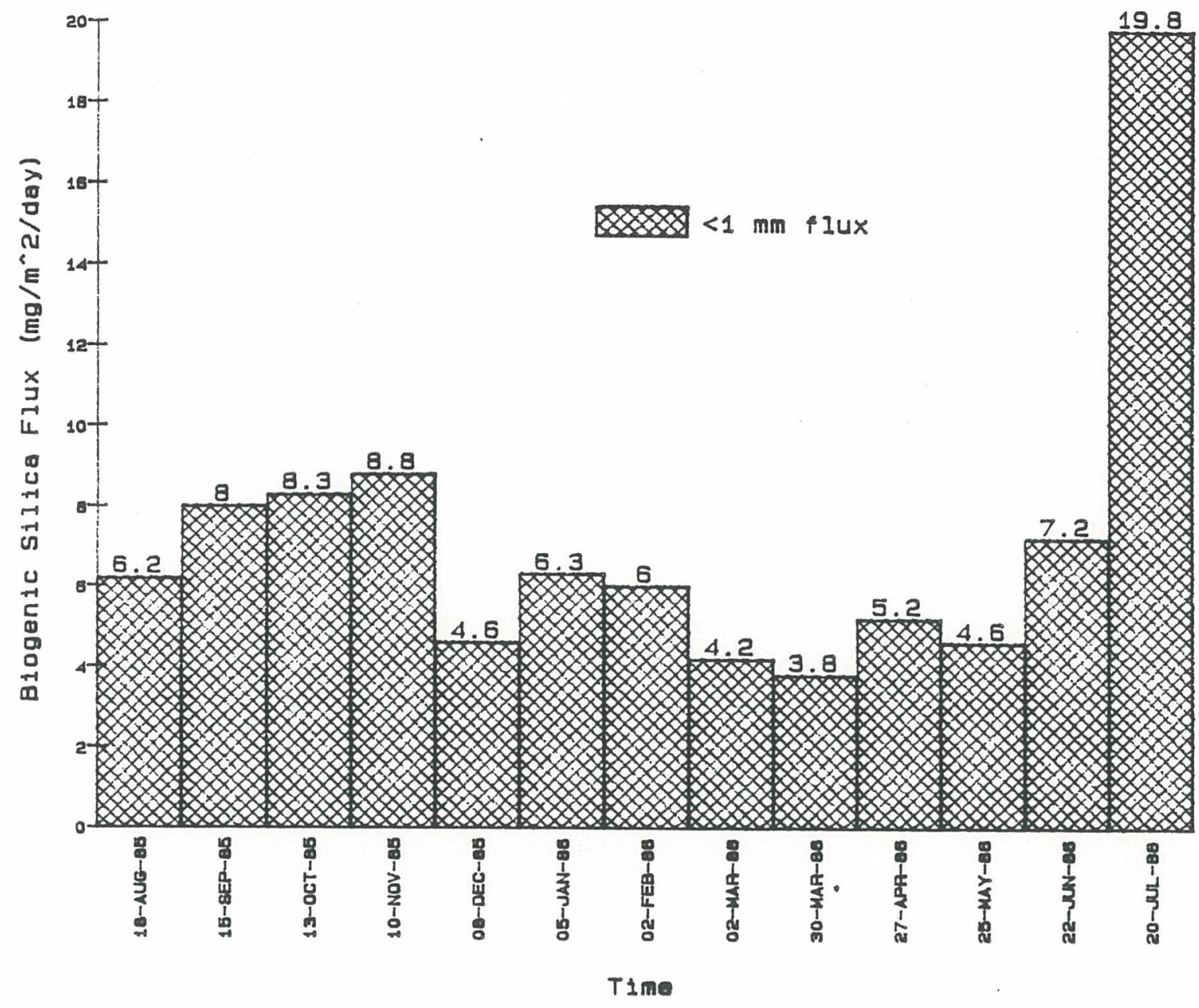

\begin{tabular}{|c|c|c|c|}
\hline $\begin{array}{l}\text { Sample } \\
\text { ID\# }\end{array}$ & $\begin{array}{l}\text { OPAL } \\
\ll 1\end{array}$ & $\begin{array}{l}\text { OPAL \% } \\
\text { NCf. }<1\end{array}$ & $\begin{array}{l}\text { OPAL \% } \\
\text { tot. }<1\end{array}$ \\
\hline $101 \mathrm{~GB} 2-3000-1$ & 6.18 & 60.40 & 31. \\
\hline $102 \mathrm{GBZ}-3000-2$ & 7.99 & 49.86 & \\
\hline 103 GB2-3000-3 & 8.34 & 49.97 & 28. \\
\hline $1046 B 2-3000-4$ & 8.81 & 48.09 & 27.1 \\
\hline $105 \mathrm{GBZ}-3000-5$ & 4.58 & 44.84 & 25.8 \\
\hline $106 \quad G B 2-3000-6$ & 6.25 & 42.21 & 22.7 \\
\hline 107 GB2-3000-7 & 5.99 & 35.96 & 20.7 \\
\hline $108 \mathrm{GBZ}-3000-8$ & 4.17 & 31.23 & 18.3 \\
\hline $109 \mathrm{~GB} 2-3000-9$ & 3.78 & 39.81 & 21.3 \\
\hline $110 \mathrm{~GB} 2-3000-10$ & 5.23 & 35.23 & 19.3 \\
\hline 111 GE2-3000-11 & 4.50 & 35.01 & 19.4 \\
\hline $112 \mathrm{GBZ}-3000-12$ & 7.12 & 47.52 & 27.5 \\
\hline $113 \mathrm{GB2}-3000-13$ & 19.83 & 56.43 & 32. \\
\hline
\end{tabular}

Flux is in $m g / m^{\wedge} 2 / d a y$.

"Ncf." = "\% of noncombustible flux".

Not enough $>1 \mathrm{~mm}$ fraction to do analysis. 


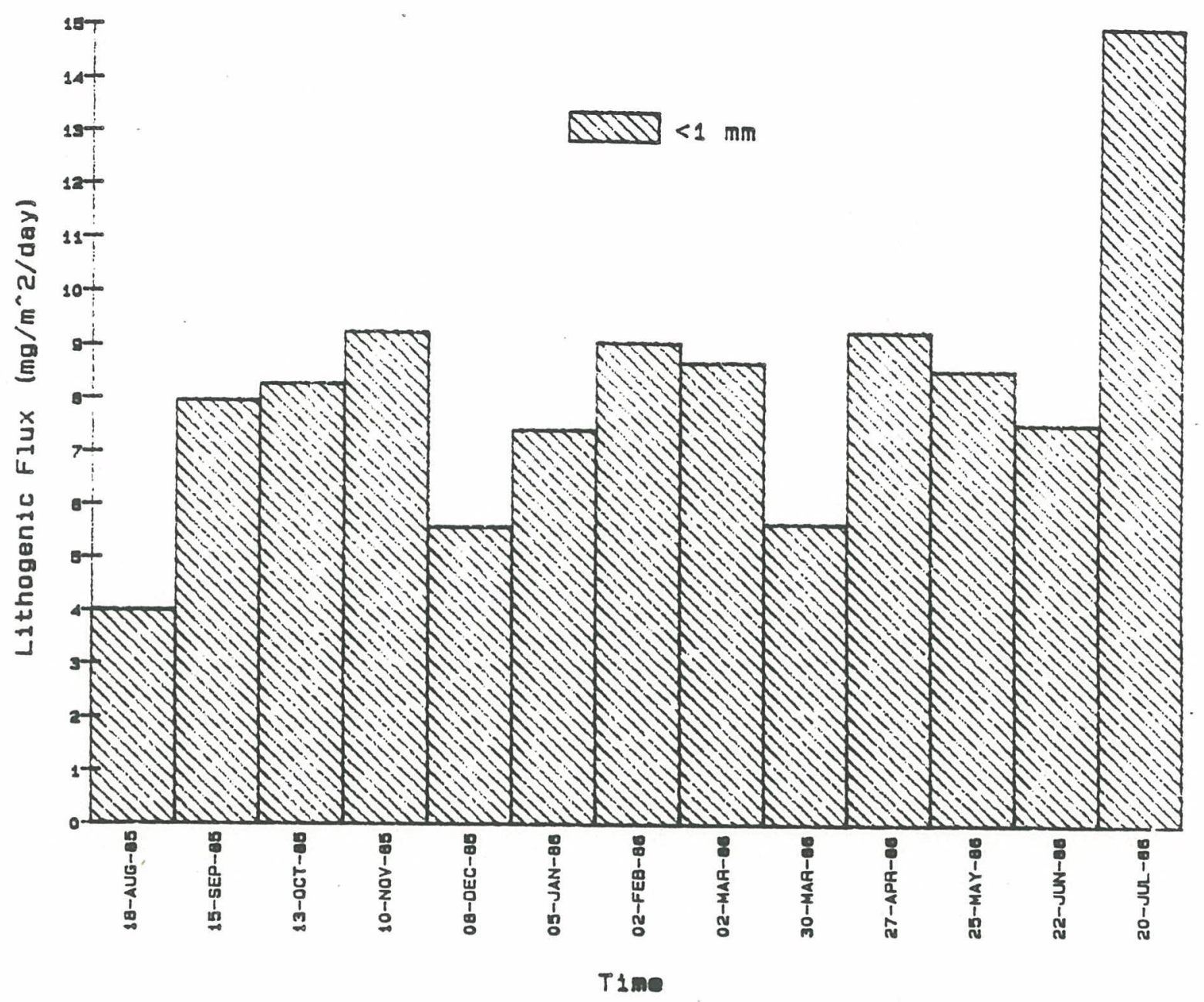

\begin{tabular}{|c|c|c|c|}
\hline $\begin{array}{l}\text { Sample } \\
\text { I.D. }\end{array}$ & $\begin{array}{l}\text { LITH } \\
\langle 1\end{array}$ & $\begin{array}{l}\text { LITH }<1 \\
\text { \%Nemb. }\end{array}$ & $\begin{array}{l}\text { LITH } \backslash 1 \\
\text { \%tot. }\end{array}$ \\
\hline $101 \mathrm{~GB} 2-3000-1$ & 4.01 & 39.22 & 20.69 \\
\hline $102 \mathrm{~GB} 2-3000-2$ & 7.97 & 49.71 & 26.20 \\
\hline $103 \mathrm{GB2}-3000-3$ & 8.27 & 49.55 & 28.52 \\
\hline $104 \mathrm{~GB} 2-3000-4$ & 9.23 & 50.39 & 28.44 \\
\hline $105682-3000-5$ & 5.58 & 54.62 & 30.54 \\
\hline 106 GB2-3000-6 & 7.41 & 50.03 & 26.93 \\
\hline $107 \mathrm{~GB} 2-3000-7$ & 9.05 & 54.30 & 31.37 \\
\hline 108 GB2-3000-8 & 8.69 & 78.70 & 38.26 \\
\hline $109 \mathrm{~GB} 2-3000-9$ & 5.66 & 59.58 & 31.96 \\
\hline $110 \mathrm{~GB} 2-3000-10$ & 9.27 & 62.41 & 34.20 \\
\hline 111 GB2-3000-11 & 8.54 & 64.99 & 36.12 \\
\hline 112 GB2-3000-12 & 7.54 & 50.32 & 29.22 \\
\hline $113 \mathrm{~GB} 2-3000-13$ & 14.93 & 42.49 & 24.61 \\
\hline
\end{tabular}

Flux is in $m g / m^{\wedge} 2 /$ day. $\%$ Ncmb. $=" \%$ of noncombustible flux".

Not enough $>1 \mathrm{~mm}$ fraction to do analysis. 
Carbon Flux at Greenland Basin 2. 3000m. 1985-86

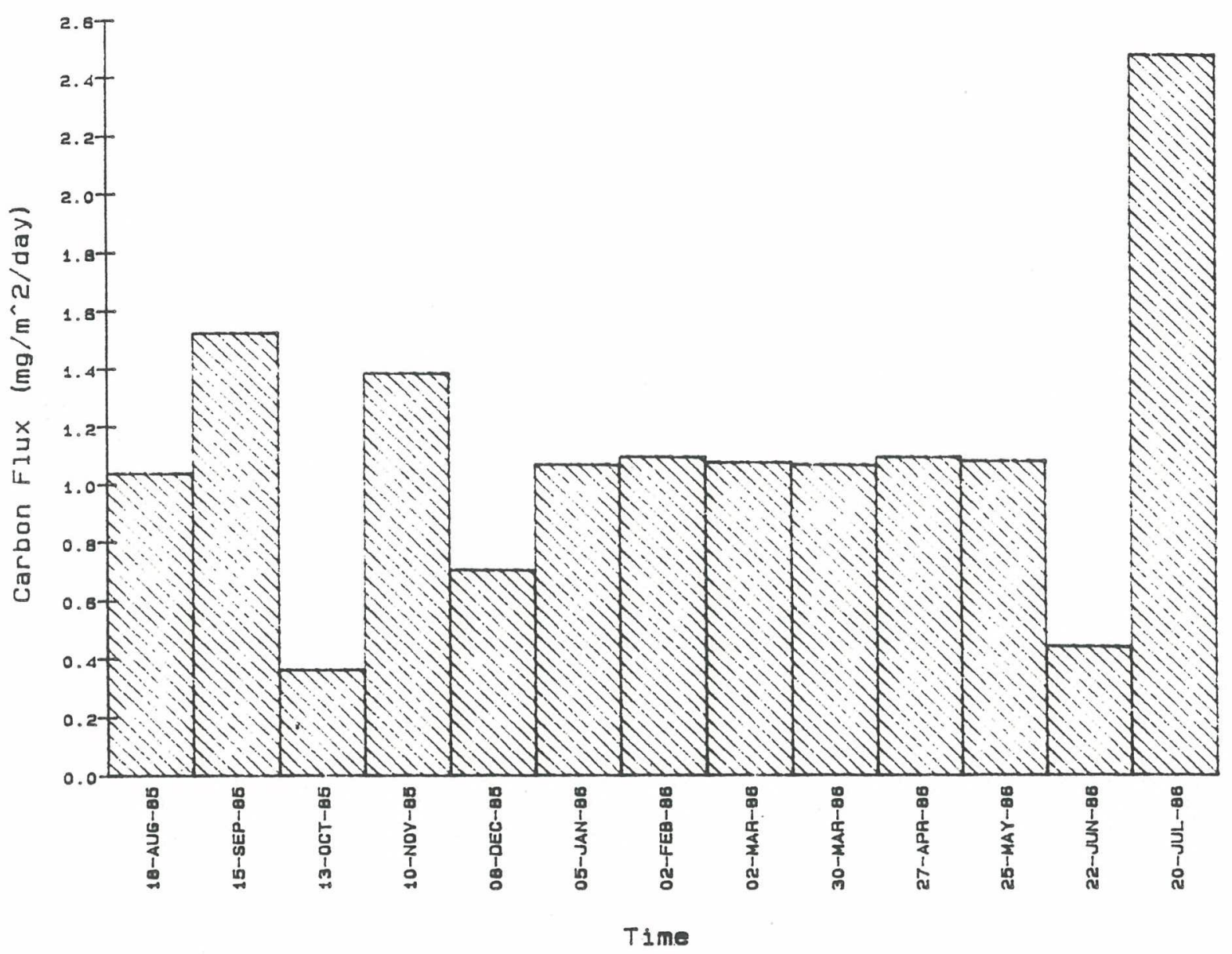

\begin{tabular}{|c|c|c|}
\hline $\begin{array}{l}\text { Gample } \\
\text { I.D. }\end{array}$ & $\begin{array}{l}\text { CRNC } \\
\vdots 1\end{array}$ & $\begin{array}{l}\text { CRNC }<1 \\
\% \text { cmb f. }\end{array}$ \\
\hline 101 GBZ-3000-1 & 1.04 & 35.06 \\
\hline 102 GE2-3000-2 & 1.52 & 35.12 \\
\hline 103 GB2-3000-3 & 0.36 & 8.99 \\
\hline 104 GEZ $-3000-4$ & 1.38 & 32.43 \\
\hline 105 GB2-3000-5 & 0.70 & 31.47 \\
\hline $106 \quad G B 2-3000-6$ & 1.07 & 25.21 \\
\hline $107 \mathrm{~GB} 2-3000-7$ & 1.09 & 34.22 \\
\hline $108 \mathrm{GBZ}-3000-8$ & 1.08 & 37.24 \\
\hline $1096 B 2-3000-9$ & 1.07 & 47.91 \\
\hline $1106 B 2-3000-10$ & 1.10 & 32.72 \\
\hline $1116 B 2-3000-11$ & 1.08 & 42.25 \\
\hline 112 GB2-3000-12 & 0.44 & 15.77 \\
\hline 113 GB2-3000-13 & 2.48 & 31.70 \\
\hline
\end{tabular}

Flux is in $m g / m$ " $2 /$ day.

"\%cmbf" = "\% of combustible flux".

Not enough $>1 \mathrm{~mm}$ fraction to do analysis. 
Nitrogen Flux at Greenland Basin 2. $3000 \mathrm{~m}, 1985-86$

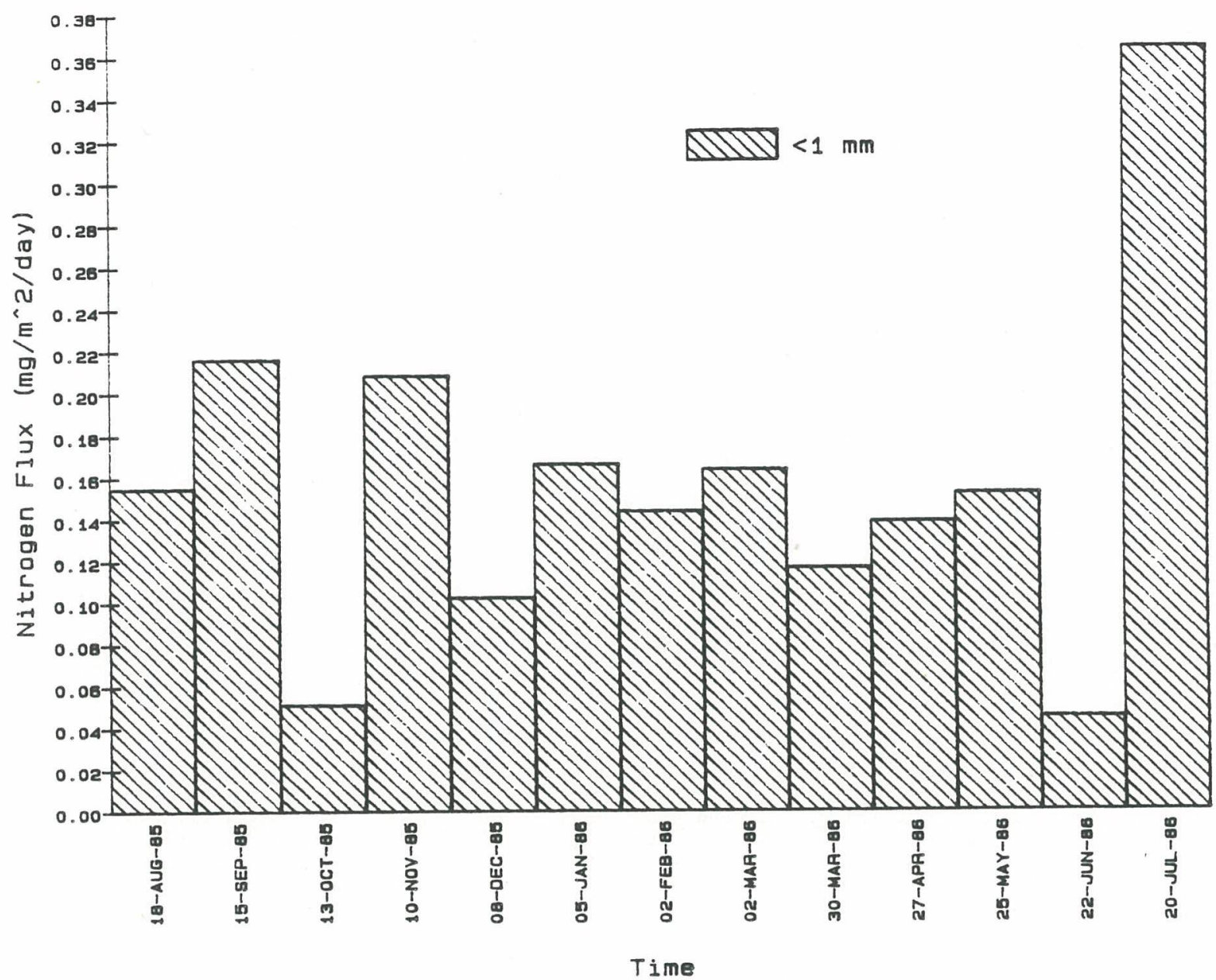

\begin{tabular}{|c|c|c|}
\hline $\begin{array}{l}\text { Sample } \\
I=0 .\end{array}$ & $\begin{array}{l}\text { NTGN } \\
\ll 1\end{array}$ & $\begin{array}{l}\text { NTGN< } 1 \\
\% \text { cmbf. }\end{array}$ \\
\hline $1016 E 2-3000-1$ & 0.15 & 5.21 \\
\hline $1026 B 2-3000-2$ & 0.22 & 4.99 \\
\hline $103682-3000-3$ & 0.05 & 1.27 \\
\hline $1046 E 2-3000-4$ & 0.21 & 4.89 \\
\hline $105682-3000-5$ & 0.10 & 4.57 \\
\hline $106 \quad 6 B 2-3000-6$ & 0.17 & 3.5 \\
\hline $107682-3000-7$ & 0.14 & 4.5 \\
\hline 108 GE2-3000-8 & 0.16 & 5.5 \\
\hline $109682-3000-9$ & 0.12 & 5.2 \\
\hline 110 GE2-3000-10 & 0.14 & 4.1 \\
\hline $111 G B 2-3000-11$ & 0.15 & 5.5 \\
\hline $112 G B 2-3000-12$ & 0.05 & 1.6 \\
\hline $113682-3000-13$ & 0.36 & 4.5 \\
\hline
\end{tabular}

Flux is in $m g / m^{*} 2 / d a y$.

"\%ombf" = "\% of combustible flux".

Not enough $1 \mathrm{~mm}$ fraction to do analysis. 
Hydrogen Flux at Greenland Basin 2. 3000m. 1985-86

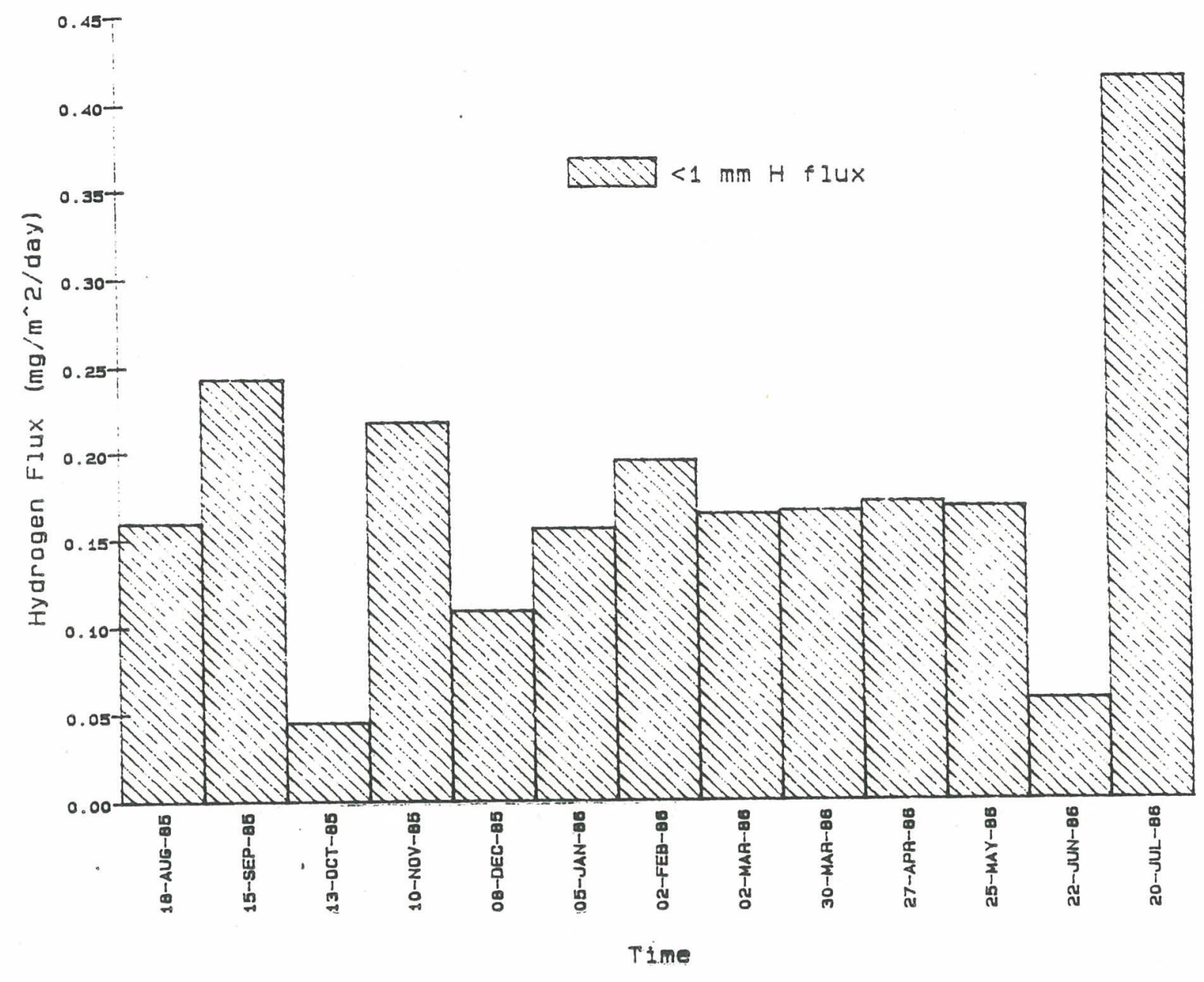

\begin{tabular}{|c|c|c|}
\hline $\begin{array}{l}\text { Sample } \\
\text { I. } 0 .\end{array}$ & $\begin{array}{l}\text { HYDC } \\
<1\end{array}$ & $\begin{array}{l}H Y D C<1 \\
\% c m b f .\end{array}$ \\
\hline $101 \mathrm{~GB} 2-3000-1$ & 0.16 & 5.39 \\
\hline $102682-3000-2$ & 0.24 & 5.60 \\
\hline $1036 B 2-3000-3$ & 0.05 & 1.12 \\
\hline 104 GB2-3000-4 & 0.22 & 5.10 \\
\hline $105682-3000-5$ & 0.11 & 4.85 \\
\hline 106 GB2-3000-6 & 0.16 & 3.68 \\
\hline 107 GB2-3000-7 & 0.20 & 6.10 \\
\hline $108 \quad 6 B 2-3000-8$ & 0.16 & 5.52 \\
\hline $109 G B 2-3000-9$ & 0.17 & 7.43 \\
\hline $110 G B 2-3000-10$ & 0.17 & 5.10 \\
\hline $1116 B 2-3000-11$ & 0.17 & 6.56 \\
\hline $112 G B 2-3000-12$ & 0.06 & 2.05 \\
\hline $1136 B 2-3000-13$ & 0.41 & 5.29 \\
\hline
\end{tabular}

Flux is in $m g / m^{\wedge} 2 / d a y$.

"\%cmbf" = "\% of combustible flux".

Not enough $>1 \mathrm{~mm}$ fraction to do analysis. 


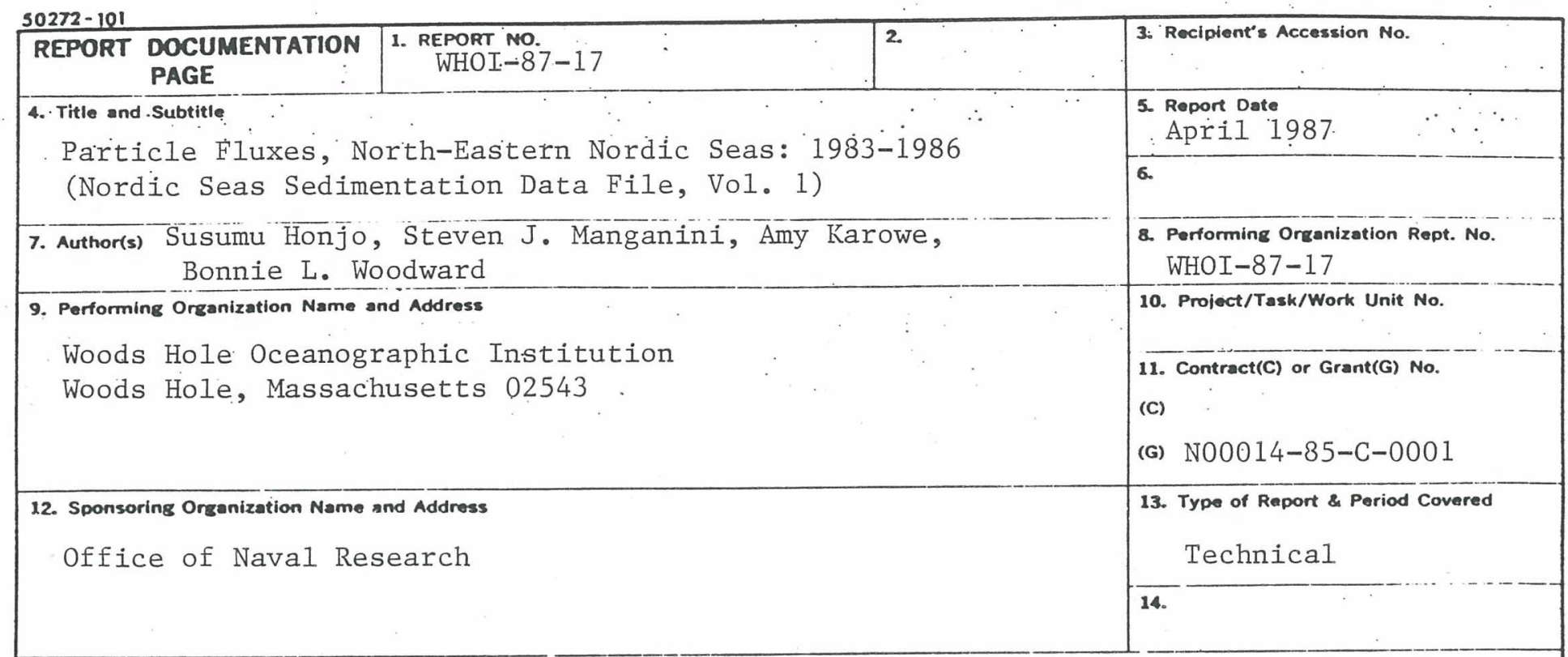

15. Supplementary Notes

This report should be cited as: Woods Hole Oceanogr. Inst. Tech. Rept. WHOI-87-17

16. Abstract (Limit: 200 words)

Seventy-nine particle flux samples were collected from 1983 to 1986 using 7 automated time-series sediment traps at 6 stations distributed in the northern and eastern portion of the Nordic Seas as part of a German/U.S. joint program on arctic sedimentation studies. Each sample represents either one month or two weeks of sedimentation at approximately $400 \mathrm{~m}$ above the sea floor. In this data file the results of laboratory analysis conducted at the Woods Hole Oceanographic Institution, U.S.A. of the main sedimentological criteria: total mass, carbonate, opal, combustible, organic carbon, nitrogen, and lithogenic mass are presented in both tabular and histogram form. Results from the southern and western portion of the Nordic Seas will be published as they become available.

17. Document Analysis a. Descriptors

1. Nordic Seas

2. Material flux

3. Sediment trap

b. Identifiers/Open-Ended Terms

c. COSATI Field/Group

\begin{tabular}{|l|l|}
\hline 18. Avallability Statemen: & 19. Socurity Class (This Report)
\end{tabular} 20. Security Clos 84

(See ANSI-239.18) See Instructions on Reverse 22. Price 


\section{DOCUMENT LIBRARY}

November 21, 1986

\section{Distribution List for Technical Report Exchange}

Institute of Marine Sciences Library

University of Alaska

O'Neill Building

905 Koyukuk Ave., North

Fairbanks, AK

Attn: Stella Sanchez-Wade

Documents Section

Scripps Institution of Oceanography

Library, Mail Code C-075C

La Jolla, CA 92093

Hancock Library of Biology \& Oceanography

Alan Hancock Laboratory

University of Southern California

University Park

Los Angeles, CA 90089-0371

Gifts \& Exchanges

Library

Bedford Institute of Oceanography

P.O. Box 1006

Dartmouth, NS, B2Y 4A2, CANADA

Office of the International Ice Patrol

c/o Coast Guard R \& D Center

Avery Point

Groton, CT 06340

Library

Physical Oceanographic Laboratory

Nova University

8000 N. Ocean Drive

Dania, FL 33304

NOAA/EDIS Miami Library Center 4301 Rickenbacker Causeway

Miami, FL 33149

Library

Skidaway Institute of Oceanography

P.O. Box 13687

Savannah, GA 31416

Institute of Geophysics

University of Hawaii

Library Room 252

2525 Correa Road

Honolulu, HI 96822

Library

Chesapeake Bay Institute

4800 Atwell Road

Shady Side, MD 20876
MIT Libraries

Serial Journal Room 14E-210

Cambridge, MA 02139

Director, Ralph M. Parsons Laboratory

Room 48-311

MIT

Cambridge, MA 02139

Marine Resources Information Center

Bldg. E38-320

MIT

Cambridge, MA 02139

Library

Lamont-Doherty Geological Observatory

Colombia University

Palisades, NY 10964

Library

Serials Department

Oregon State University

Corvallis, OR 97331

Pell Marine Science Library

University of Rhode Island

Narragansett Bay Campus

Narragansett, RI 02882

Working Collection

Texas A\&M University

Dept. of Oceanography

College Station, TX 77843

Library

Virginia Institute of Marine Science

Gloucester Point, VA 23062

Fisheries-Oceanography Library

151 Oceanography Teaching Bldg.

University of Washington

Seattle, WA 98195

Library

R.S.M.A.S.

University of Miami

4600 Rickenbacker Causeway

Miami, FL 33149

Maury Oceanographic Library

Naval Oceanographic Office

Bay St. Louis

NSTL, MS 39522-5001

ATTN: Code 4601 